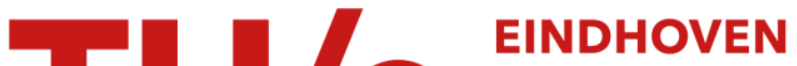 UNIVERSITY OF TECHNOLOGY
}

\section{The survival probability for critical spread-out oriented percolation above 4+1 dimensions, Il. Expansion}

\section{Citation for published version (APA):}

Hofstad, van der, R. W., Hollander, den, W. T. F., \& Slade, G. (2007). The survival probability for critical spreadout oriented percolation above 4+1 dimensions, II. Expansion. Annales de l'institut Henri Poincare (B):

Probability and Statistics, 43(5), 509-570. https://doi.org/10.1016/j.anihpb.2006.09.002

DOI:

10.1016/j.anihpb.2006.09.002

Document status and date:

Published: 01/01/2007

\section{Document Version:}

Publisher's PDF, also known as Version of Record (includes final page, issue and volume numbers)

\section{Please check the document version of this publication:}

- A submitted manuscript is the version of the article upon submission and before peer-review. There can be important differences between the submitted version and the official published version of record. People interested in the research are advised to contact the author for the final version of the publication, or visit the $\mathrm{DOI}$ to the publisher's website.

- The final author version and the galley proof are versions of the publication after peer review.

- The final published version features the final layout of the paper including the volume, issue and page numbers.

Link to publication

\section{General rights}

Copyright and moral rights for the publications made accessible in the public portal are retained by the authors and/or other copyright owners and it is a condition of accessing publications that users recognise and abide by the legal requirements associated with these rights.

- Users may download and print one copy of any publication from the public portal for the purpose of private study or research.

- You may not further distribute the material or use it for any profit-making activity or commercial gain

- You may freely distribute the URL identifying the publication in the public portal.

If the publication is distributed under the terms of Article 25fa of the Dutch Copyright Act, indicated by the "Taverne" license above, please follow below link for the End User Agreement:

www.tue.nl/taverne

Take down policy

If you believe that this document breaches copyright please contact us at:

openaccess@tue.nl

providing details and we will investigate your claim. 


\title{
The survival probability for critical spread-out oriented percolation above $4+1$ dimensions. II. Expansion
}

\author{
Remco van der Hofstad ${ }^{\mathrm{a}, *}$, Frank den Hollander ${ }^{\mathrm{b}, \mathrm{c}}$, Gordon Slade ${ }^{\mathrm{d}}$ \\ a Department of Mathematics and Computer Science, Eindhoven University of Technology, P.O. Box 513, 5600 MB Eindhoven, The Netherlands \\ b Mathematical Institute, Leiden University, P.O. Box 9512, 2300 RA Leiden, The Netherlands \\ ${ }^{\mathrm{c}}$ EURANDOM, P.O. Box 513, $5600 \mathrm{MB}$ Eindhoven, The Netherlands \\ d Department of Mathematics, University of British Columbia, Vancouver, BC V6T 1Z2, Canada
}

Received 22 September 2005; received in revised form 12 July 2006; accepted 12 September 2006

Available online 15 December 2006

\begin{abstract}
We derive a lace expansion for the survival probability for critical spread-out oriented percolation above $4+1$ dimensions, i.e., the probability $\theta_{n}$ that the origin is connected to the hyperplane at time $n$, at the critical threshold $p_{c}$. Our lace expansion leads to a non-linear recursion relation for $\theta_{n}$, with coefficients that we bound via diagrammatic estimates. This lace expansion is for point-to-plane connections and differs substantially from previous lace expansions for point-to-point connections. In particular, to be able to deduce the asymptotics of $\theta_{n}$ for large $n$, we need to derive the recursion relation up to quadratic order.

The present paper is Part II in a series of two papers. In Part I, we use the recursion relation and the diagrammatic estimates to prove that $\lim _{n \rightarrow \infty} n \theta_{n}=1 / B \in(0, \infty)$, and also deduce consequences of this asymptotics for the geometry of large critical clusters and for the incipient infinite cluster.

() 2006 Elsevier Masson SAS. All rights reserved.
\end{abstract}

Keywords: Oriented percolation; Lace expansion; Survival probability; Critical exponent; Nonlinear recursion

\section{Introduction and results}

For oriented bond percolation on $\mathbb{Z}^{d} \times \mathbb{Z}_{+}$with parameter $p$, the survival probability $\theta_{n}=\theta_{n}(p)$ at time $n \in \mathbb{Z}_{+}$is the probability that there exists an $x \in \mathbb{Z}^{d}$ such that $(0,0)$ is connected to $(x, n)$. In the oriented setting, it is known that there is no percolation at the critical threshold $p=p_{c}[2,4]$, so that $\lim _{n \rightarrow \infty} \theta_{n}\left(p_{c}\right)=0$. Our goal is to study the manner in which $\theta_{n}\left(p_{c}\right)$ tends to zero as $n \rightarrow \infty$ when $d>4$.

In the present paper, we derive a lace expansion for $\theta_{n}(p)$, valid in all dimensions $d \geqslant 1$ and for quite general models of oriented percolation. This lace expansion gives a nonlinear recursion relation for $\theta_{n}(p)$. If the expansion is to be useful, then the coefficients in the recursion relation need to be estimated. We prove estimates valid at $p=p_{c}$ in dimensions $d>4$, for sufficiently "spread-out" oriented bond percolation (defined below), with the degree to which connections are spread out in space parameterised by a sufficiently large $L \in \mathbb{N}$.

\footnotetext{
* Corresponding author.

E-mail addresses: rhofstad@win.tue.nl (R. van der Hofstad), denholla@math.leidenuniv.nl (F. den Hollander), slade@math.ubc.ca (G. Slade).
} 
In Part I [7], we have shown how these results can be used in an induction analysis for the recursion relation to conclude that there is a constant $B=B(d, L)$ such that, as $n \rightarrow \infty$,

$$
\theta_{n}\left(p_{c}\right)-\theta_{n+1}\left(p_{c}\right)=\frac{1}{B n^{2}}\left[1+\mathrm{O}\left(n^{-1} \log n\right)+L^{-d} \mathrm{O}\left(\delta_{n}\right)\right] \text { for } d>4 \text { and } L \text { sufficiently large, }
$$

where

$$
\delta_{n}= \begin{cases}n^{-(d-4) / 2} \log n & (4<d<6), \\ n^{-1} \log ^{2} n & (d=6), \\ n^{-1} \log n & (d>6) .\end{cases}
$$

In other words, the critical extinction probability $\theta_{n}\left(p_{c}\right)-\theta_{n+1}\left(p_{c}\right)$, which is the probability that the cluster of the origin survives to time $n$ but not to time $n+1$, is asymptotic to $1 /\left(B n^{2}\right)$ as $n \rightarrow \infty$, with accurate error bounds. By summing over $n$, we conclude that

$$
\theta_{n}\left(p_{c}\right)=\frac{1}{B n}\left[1+\mathrm{O}\left(n^{-1} \log n\right)+L^{-d} \mathrm{O}\left(\delta_{n}\right)\right] \text { for } d>4 \text { and } L \text { sufficiently large, }
$$

which is the main conclusion of Part I. In terms of the critical exponent $\rho$, defined by the conjecture that $\theta_{n}\left(p_{c}\right)$ behaves like $n^{-1 / \rho}$ as $n \rightarrow \infty$, (1.3) implies that $\rho$ exists and is equal to 1 , for $d>4$ and $L$ sufficiently large.

Also in Part I, interesting consequences for the geometry of large critical clusters and for the incipient infinite cluster were deduced from (1.3), using results from [8]. In particular, (1.3) implies that two constructions for the incipient infinite cluster coincide and that, conditionally on survival up to time $n$, the number of vertices to which the origin is connected at time $n$ scales like $n$ times an exponential random variable.

\subsection{The model}

The spread-out oriented percolation model is defined as follows. Let $\mathbb{Z}_{+}=\{n \in \mathbb{Z}: n \geqslant 0\}$. Consider the graph with vertices $\mathbb{Z}^{d} \times \mathbb{Z}_{+}$and with directed bonds $((x, n),(y, n+1))$, for $n \in \mathbb{Z}_{+}$and $x, y \in \mathbb{Z}^{d}$. Let $D$ be a fixed function $D: \mathbb{Z}^{d} \rightarrow[0,1]$, satisfying

$$
\sum_{x \in \mathbb{Z}^{d}} D(x)=1 .
$$

The function $D$ will be assumed to be invariant under the symmetries of $\mathbb{Z}^{d}$ (permutation and reflection of coordinates). Let $p \in\left[0,\|D\|_{\infty}^{-1}\right]$, where $\|\cdot\|_{\infty}$ denotes the supremum norm, so that $p D(x) \leqslant 1$ for all $x \in \mathbb{Z}^{d}$. We associate to each directed bond $((x, n),(y, n+1))$ an independent random variable taking the value 1 with probability $p D(y-x)$ and the value 0 with probability $1-p D(y-x)$. We say that a bond is occupied when the corresponding random variable is 1 and vacant when it is 0 . Note that $p$ is not a probability. Rather, $p$ is the average number of occupied bonds from a given vertex. The joint probability distribution of the bond variables will be denoted by $\mathbb{P}_{p}$ and the corresponding expectation by $\mathbb{E}_{p}$, with the parameter $p$ usually suppressed from the notation.

For the diagrammatic estimates, we need to make further assumptions on $D$. We will refer to the assumptions on $D$ in the previous paragraph as the weak assumptions on $D$. We define the spread-out model of oriented percolation to be the model in which $D$ obeys the weak assumptions together with Assumption D in [13, Section 1.2] (whose precise form is not important for the present paper), and [14, Eq. (1.2)]. Assumption D in [13, Section 1.2] involves a parameter $L \in \mathbb{N}$, which serves to spread out the connections and which will be taken to be fixed and large. A simple and basic example is

$$
D(x)= \begin{cases}(2 L+1)^{-d} & \text { if }\|x\|_{\infty} \leqslant L, \\ 0 & \text { otherwise. }\end{cases}
$$

In this example, the bonds are given by $((x, n),(y, n+1))$ with $\|x-y\|_{\infty} \leqslant L$, and a bond is occupied with probability $p(2 L+1)^{-d}$. Assumption $\mathrm{D}$ also allows for certain infinite range models. For the spread-out model, we will use

$$
\beta=L^{-d}
$$

as a small parameter. Assumption D implies that there is a finite positive constant $C$ such that

$$
\sup _{x \in \mathbb{Z}^{d}} D(x) \leqslant C \beta .
$$


We say that $(x, n)$ is connected to $(y, m)$, and write $(x, n) \rightarrow(y, m)$, if there is an oriented path from $(x, n)$ to $(y, m)$ consisting of occupied bonds. Note that this is only possible when $m \geqslant n$. By convention, $(x, n)$ is connected to itself. We write

$$
C(x, n)=\left\{(y, m) \in \mathbb{Z}^{d} \times \mathbb{Z}_{+}:(x, n) \rightarrow(y, m)\right\}
$$

to denote the forward cluster of $(x, n)$. We also write $(x, n) \rightarrow m$ to denote the event that there is a $y \in \mathbb{Z}^{d}$ such that $(x, n) \rightarrow(y, m)$.

The event $\{(0,0) \rightarrow \infty\}$ is the event that $\{(0,0) \rightarrow n\}$ occurs for all $n$. There is a critical threshold $p_{c} \in(0, \infty)$ such that the event $\{(0,0) \rightarrow \infty\}$ has probability zero for $p \leqslant p_{c}$ and has positive probability for $p>p_{c}$. The parametrisation we have chosen is convenient, since for the spread-out model it is known that

$$
p_{c}=1+c L^{-d}+\mathrm{O}\left(L^{-d-1}\right) \quad \text { as } L \rightarrow \infty,
$$

for $d>4$, with the positive constant $c$ given explicitly in terms of the Green function for the random walk with step distribution $D[10]$.

The survival probability at time $n$ is defined by

$$
\theta_{n}(p)=\mathbb{P}_{p}((0,0) \rightarrow n) .
$$

General results of $[2,4]$ imply that $\lim _{n \rightarrow \infty} \theta_{n}\left(p_{c}\right)=0$. For the spread-out model in dimension $d>4$, with $L$ sufficiently large, the same conclusion was shown in [1] to follow from the triangle condition. The triangle condition was verified under the above hypotheses in [14,16], yielding an alternate proof that $\lim _{n \rightarrow \infty} \theta_{n}\left(p_{c}\right)=0$ for $d>4$, and $L$ sufficiently large.

\subsection{Main theorem}

For $n \in \mathbb{Z}_{+}, x \in \mathbb{Z}^{d}$, and $p \in\left[0,\|D\|_{\infty}^{-1}\right]$, we define the two-point function

$$
\tau_{n}(x)=\mathbb{P}_{p}((0,0) \rightarrow(x, n)) .
$$

We write

$$
\tau_{n}=\sum_{x \in \mathbb{Z}^{d}} \tau_{n}(x)
$$

for the expected number of vertices in $C(0,0)$ at time $n$. The lace expansion for the two-point function [5] (see also [14]) yields a recursion relation for $\tau_{n}$, which reads

$$
\tau_{n}=\sum_{m=0}^{n-1} \pi_{m} p \tau_{n-m-1}+\pi_{n},
$$

where $\left(\pi_{m}\right)$ are certain $p$-dependent coefficients. In fact, (1.13) uniquely defines $\left(\pi_{m}\right)$, but the lace expansion provides a useful representation for $\left(\pi_{m}\right)$. In [14, Proposition 2.2], this representation was used to prove that $\pi_{0}=1, \pi_{1}=0$ and that there exists a finite positive constant $C_{\pi}$ such that

$$
\left|\pi_{m}\right| \leqslant \frac{C_{\pi} \beta}{(m+1)^{d / 2}} \quad\left(p=p_{c}, m \geqslant 2\right),
$$

for the spread-out model in dimensions $d>4$, with $\beta$ of (1.6) sufficiently small. In addition, under the same assumptions, it is shown in [14, Eq. (2.11)] that

$$
\sum_{m=0}^{\infty} \pi_{m} p_{c}=1
$$

In the present paper, we obtain a lace expansion for the survival probability $\theta_{n}$, with good bounds valid for the spread-out model in dimensions $d>4$ at $p=p_{c}$. Our main result is the following theorem. In its statement, we use the notation

$$
\Delta_{n}= \begin{cases}n^{-(d-4) / 2} \log n & (4<d<6) \\ n^{-1} \log n & (d=6) \\ n^{-1} & (d>6)\end{cases}
$$


Theorem 1.1 (Lace expansion and diagrammatic estimates).

(i) For $d \geqslant 1, p \in\left[0,\|D\|_{\infty}^{-1}\right]$, and $n \geqslant 1$, and under the weak assumption on $D$,

$$
\theta_{n}(p)=\sum_{m=0}^{n-1} \pi_{m}(p) p \theta_{n-1-m}(p)-\sum_{m_{1}=1}^{\lfloor n / 2\rfloor} \sum_{m_{2}=m_{1}}^{n} \phi_{m_{1}, m_{2}}(p) \theta_{n-m_{1}}(p) \theta_{n-m_{2}}(p)+e_{n}(p),
$$

where $\left(\pi_{m}\right)$ are as in (1.13), and $\left(\phi_{m_{1}, m_{2}}\right)$ and $\left(e_{n}\right)$ are given by explicit formulas (see Sections 4,5$)$.

(ii) For the spread-out model in dimensions $d>4$, at the critical value $p=p_{c}$, there are finite positive constants $C_{\phi}, C_{e}$, and $\beta_{0}$ such that, for $0<\beta \leqslant \beta_{0}$, the coefficients $\left(\phi_{m_{1}, m_{2}}\right)$ and the error terms $\left(e_{n}\right)$ satisfy the following estimates:

- $\phi_{1,1}\left(p_{c}\right)=\frac{1}{2} p_{c}^{2} \sum_{x \in \mathbb{Z}^{d}} D(x)(1-D(x))=\frac{1}{2}[1+\mathrm{O}(\beta)]$ and, for $m_{2} \geqslant m_{1} \geqslant 1$ such that $\left(m_{1}, m_{2}\right) \neq(1,1)$,

$$
\left|\phi_{m_{1}, m_{2}}\left(p_{c}\right)\right| \leqslant C_{\phi} \beta\left(m_{1}+1\right)^{-(d-2) / 2}\left(m_{2}-m_{1}+1\right)^{-(d-2) / 2} .
$$

- If $\theta_{m}\left(p_{c}\right) \leqslant C_{\theta}(m+1)^{-1}$ for $0 \leqslant m \leqslant n$ and some $C_{\theta} \geqslant 1$, then

$$
\left|e_{n+1}\left(p_{c}\right)\right| \leqslant C_{e} C_{\theta}^{3}(n+1)^{-2}\left[(n+1)^{-1}+\beta \Delta_{n+1}\right] .
$$

Note that the diagrammatic estimate (1.19) for $e_{n+1}$, which is the error term in (1.17) for $\theta_{n+1}$, assumes a bound in the recursion relation for $\theta_{m}$ only for $0 \leqslant m \leqslant n$. This is precisely what opens up the possibility of the inductive analysis employed in Part I. Namely, in Part I, (1.1) is deduced from Theorem 1.1 by applying an induction analysis to (1.17), which makes use of the bounds in (1.14), (1.18) and (1.19) in order to moderate the coefficients of the recursion.

When we derive (1.17) in Sections 2-5, we will fix an arbitrary $p \in\left[0,\|D\|_{\infty}^{-1}\right]$ and assume only the weak assumption on $D$. In Sections 6-8, where we prove the diagrammatic estimates (1.18), (1.19), we will specialise to the spread-out model with $d>4, p=p_{c}$, and small $\beta$.

We expect that Theorem 1.1 has implications also for the critical contact process in spatial dimension $d>4$. Indeed, it has been shown in [9] that the lace expansion for the two-point function can be applied to the oriented percolation model resulting from time discretisation of the contact process. We expect that part (i) of the theorem can be applied similarly to study the survival probability for the critical contact process, in conjunction with a suitable modification of part (ii). The extension of our results to the contact process will be taken up in $[11,12]$.

\subsection{The constant $B$}

It was shown in [7, Eq. (1.36)] that the constant $B$ in (1.3) is given by

$$
B=\frac{\sum_{m_{1}=1}^{\infty} \sum_{m_{2}=m_{1}}^{\infty} \phi_{m_{1}, m_{2}}\left(p_{c}\right)}{1+p_{c} \sum_{m=2}^{\infty} m \pi_{m}\left(p_{c}\right)} \text {. }
$$

It follows from (1.9), (1.14) and (1.18) that $B<\infty$ for $d>4$ and $\beta$ sufficiently small, with $B=\frac{1}{2}+\mathrm{O}(\beta)$ as $\beta \downarrow 0$.

The survival probability $\hat{\theta}_{n}$ of a Galton-Watson branching process whose offspring distribution has mean 1 , variance $\hat{\sigma}^{2}$, and finite third moment, obeys the simple recursion relation

$$
\hat{\theta}_{n}=\hat{\theta}_{n-1}-\frac{\hat{\sigma}^{2}}{2} \hat{\theta}_{n-1}^{2}+\hat{e}_{n}
$$

where $e_{n}=\mathrm{O}\left(\hat{\theta}_{n-1}^{3}\right)$. This leads to the conclusion that $\lim _{n \rightarrow \infty} n \hat{\theta}_{n}=2 \hat{\sigma}^{-2}$. We sketch the proof of these wellknown facts in Part I. Consider the branching process with offspring distribution $\sum_{x} I_{x}$, where the $I_{x}$ are independent Bernoulli random variables with parameter $D(x)$. This offspring distribution has mean 1 , by the normalisation assumption for $D$, and has variance $\hat{\sigma}^{2}=\sum_{x} D(x)(1-D(x))=1+\mathrm{O}(\beta)$, as $L \rightarrow \infty$ in the spread-out model, by (1.7). We regard the critical spread-out oriented percolation model in dimensions $d>4$ as a small perturbation of this critical branching process - the former allows at most one particle per vertex, whereas the latter allows multiple occupancy. The recursion relation (1.17) can be viewed as a perturbation of (1.21). The fact that $B=\frac{1}{2}[1+\mathrm{O}(\beta)]$ as $L \rightarrow \infty$ 
shows that the solution to (1.17) for the spread-out model remains close to the solution of (1.21), to leading order, for $L$ large.

Let $N_{n}$ denote the number of vertices in $C(0,0)$ at time $n$, when $p=p_{c}$, and define the constants $A$ and $V$ by

$$
A=\lim _{n \rightarrow \infty} \mathbb{E}_{p_{c}}\left[N_{n}\right], \quad V=\lim _{n \rightarrow \infty} \frac{1}{A^{3} n} \mathbb{E}_{p_{c}}\left[N_{n}^{2}\right] .
$$

It is part of the results in [14] that these constants exist when $d>4$ and $L$ is sufficiently large. It is shown in [8] that, given $n \theta_{n}\left(p_{c}\right) \rightarrow 1 / B$ (which follows from (1.3)),

$$
B=\frac{A V}{2} \text {. }
$$

It is shown in [14, Eqs. (2.12) and (2.49)] that

$$
A=\frac{1}{p_{c}+p_{c}^{2} \sum_{m=2}^{\infty} m \pi_{m}\left(p_{c}\right)}, \quad V=\sum_{m_{1}=2}^{\infty} \sum_{m_{2}=2}^{\infty} \hat{\psi}_{m_{1}, m_{2}}(0,0),
$$

where $\left(\hat{\psi}_{m_{1}, m_{2}}\right)$ are coefficients arising in the lace expansion for the critical three-point function

$$
\tau_{n_{1}, n_{2}}\left(x_{1}, x_{2}\right)=\mathbb{P}_{p_{c}}\left((0,0) \rightarrow\left(x_{1}, n_{1}\right),(0,0) \rightarrow\left(x_{2}, n_{2}\right)\right) .
$$

It follows from (1.20) and (1.23), (1.24) that

$$
V=\sum_{m_{1}=2}^{\infty} \sum_{m_{2}=2}^{\infty} \hat{\psi}_{m_{1}, m_{2}}(0,0)=2 p_{c} \sum_{m_{1}=1}^{\infty} \sum_{m_{2}=m_{1}}^{\infty} \phi_{m_{1}, m_{2}}\left(p_{c}\right) .
$$

This implies that the coefficients $\left(\phi_{m_{1}, m_{2}}\right)$ in our lace expansion for the survival probability are related to those appearing in the lace expansion for the three-point function. However, our approach does not reveal an explicit relation between $\hat{\psi}_{m_{1}, m_{2}}(0,0)$ and $\phi_{m_{1}, m_{2}}$ for fixed $m_{1}, m_{2}$. In [11], an alternate expansion for the three-point function is derived, which is quite different from the expansion of [14] and closer in spirit to the expansion derived here for the survival probability. The expansion of [11] leads to a direct proof that

$$
V=2 p_{c} \sum_{m_{1}=1}^{\infty} \sum_{m_{2}=m_{1}}^{\infty} \phi_{m_{1}, m_{2}}\left(p_{c}\right) \text {. }
$$

\subsection{Organisation}

The remainder of the paper is devoted to the proof of Theorem 1.1. The proof is divided into two main parts: (a) the derivation of the expansion (1.17) for $\theta_{n}$, and (b) the proof of the diagrammatic estimates (1.18), (1.19) for the expansion coefficients. The basic steps in the proof of each part are as follows.

(a) Derivation of the lace expansion (1.17). The starting point for the expansion is the percolation lace expansion of [5] for the two-point function. This expansion was applied to oriented percolation in [14], where a derivation of (1.13) can be found. We will extend this lace expansion for the two-point function (a point-to-point expansion) to a lace expansion for the survival probability (a point-to-plane expansion). There are alternate expansions for the two-point function of oriented percolation, due to [16] and [17] (see [18] for a description of all three expansions), but we do not know how to use these alternate expansions to obtain an expansion for the survival probability.

The expansion of [5] is based on a factorisation lemma, which we isolate in Section 2. In Section 3, we extract the linear term in (1.17) using a relatively minor extension of the lace expansion for the two-point function. This produces an equation

$$
\theta_{n}=\sum_{m=0}^{n-1} \pi_{m} p \theta_{n-1-m}+\chi_{n}
$$

where the term $\chi_{n}$ involves configurations with two connections to the hyperplane at time $n$. These two connections lead to the quadratic term in (1.17), but two further expansions are required to obtain the two factors $\theta_{n-m_{1}} \theta_{n-m_{2}}$ in (1.17). 
The first of these expansions for $\chi_{n}$ is the most delicate and novel part of our method. A crucial role is played by a random set $\mathcal{P}_{A}$ of bonds, which is defined in Section 4 for any fixed subset $A$ of $\mathbb{Z}^{d} \times \mathbb{Z}_{+}$. Using $\mathcal{P}_{A}$, we extract a factor $\theta_{n-m_{1}}$ from $\chi_{n}$ in Section 4, completing the first expansion for $\chi_{n}$. Then, in Section 5, we perform a second expansion for $\chi_{n}$ to extract an additional factor $\theta_{n-m_{2}}$. Our treatment of this second expansion is different in spirit than the expansion methods used in [6,14], and is simpler due to a careful use of independence that is due to the orientation.

This part of the argument applies for general $p$ and $d$, and makes only the weak assumption on $D$.

(b) The diagrammatic estimates (1.18), (1.19). As is usual in lace expansion analyses, we will prove (1.18), (1.19) by bounding $\phi_{m_{1}, m_{2}}$ and $e_{n+1}$ by diagrams of the same character as the Feynman diagrams of physics, i.e., by sums of products of two-point functions and survival probabilities. The two-point functions are bounded using estimates proved in [14], and the survival probabilities are bounded using the assumption on $\theta_{m}\left(p_{c}\right)$ given above (1.19).

The first step in this procedure is carried out in Section 6, where we generalise the bound on $\pi_{m}$ of [14], stated above in (1.14), and prove related bounds on $\chi_{n}$. The bounds on $\phi_{m_{1}, m_{2}}$ and $e_{n+1}$ are in terms of diagrams that are built from the diagrams encountered in Section 6 using certain diagrammatic constructions. Using these, in Section 7, we complete the proof of the bound (1.18) on $\phi_{m_{1}, m_{2}}$, and in Section 8, we complete the proof of the bound (1.19) on $e_{n+1}$.

This part of the argument is for the spread-out model. It relies on $d>4$ and small $\beta$, and the bounds we obtain apply at $p=p_{c}$.

\section{The Factorisation Lemma}

This section contains some preliminaries that will be crucial in the expansion for the survival probability. The main result is the Factorisation Lemma stated in Lemma 2.2 below. Throughout the rest of the paper, we write

$$
\Lambda=\mathbb{Z}^{d} \times \mathbb{Z}_{+}
$$

and we use bold letters such as $\boldsymbol{x}, \boldsymbol{y}, \boldsymbol{z}$ for elements of $\Lambda$. To be able to state the Factorisation Lemma, we need some definitions.

\section{Definition 2.1.}

(i) Given a (deterministic or random) set of vertices $A$ and a bond configuration $\omega$, we define $\omega_{A}$, the restriction of $\omega$ to $A$, to be

$$
\omega_{A}(\{\boldsymbol{x}, \boldsymbol{y}\})= \begin{cases}\omega(\{\boldsymbol{x}, \boldsymbol{y}\}) & \text { if } \boldsymbol{x}, \boldsymbol{y} \in A, \\ 0 & \text { otherwise }\end{cases}
$$

for every $\boldsymbol{x}, \boldsymbol{y}$ such that $\{\boldsymbol{x}, \boldsymbol{y}\}$ is a bond. In other words, $\omega_{A}$ is obtained from $\omega$ by making every bond that does not have both endpoints in $A$ vacant.

(ii) Given a (deterministic or random) set of vertices $A$ and an event $E$, we say that $E$ occurs in $A$, and write $\{E$ in $A\}$, if $\omega_{A} \in E$. In other words, $\{E$ in $A\}$ means that $E$ occurs on the (possibly modified) configuration in which every bond that does not have both endpoints in $A$ is made vacant. We adopt the convenient convention that $\{\boldsymbol{x} \rightarrow \boldsymbol{x}$ in $A\}$ occurs if and only if $\boldsymbol{x} \in A$.

(iii) Given a bond configuration and $\boldsymbol{x} \in \Lambda$, we define $C(\boldsymbol{x})$ to be the set vertices to which $\boldsymbol{x}$ is connected, i.e., $C(\boldsymbol{x})=\{\boldsymbol{y} \in \Lambda: \boldsymbol{x} \rightarrow \boldsymbol{y}\}$. Given a bond configuration and a bond $b$, we define $\tilde{C}^{b}(\boldsymbol{x})$ to be the set of vertices $\boldsymbol{y} \in C(\boldsymbol{x})$ to which $\boldsymbol{x}$ is connected in the (possibly modified) configuration in which $b$ is made vacant.

We will often use the following easily verified rules for occurs in:

$$
\begin{aligned}
& \{E \text { in } B\} \cap\{F \text { in } B\}=\{E \cap F \text { in } B\}, \\
& \{E \text { in } B\} \cup\{F \text { in } B\}=\{E \cup F \text { in } B\}, \\
& \{E \text { in } B\}^{c}=\left\{E^{c} \text { in } B\right\} .
\end{aligned}
$$

Eqs. (2.3)-(2.5) imply that "occurs in" is well behaved under set operations. 
The following Factorisation Lemma lies at the heart of the expansion method. ${ }^{1}$ We write $I[E]$ for the indicator function of an event $E$. The statement of the Factorisation Lemma is in terms of $t w o$ independent percolation configurations. The laws of these independent configurations are indicated by subscripts, i.e., $\mathbb{E}_{0}$ denotes the expectation with respect to the first percolation configuration, and $\mathbb{E}_{1}$ denotes the expectation with respect to the second percolation configuration. We also use the same subscripts for random variables, to indicate which law describes their distribution. Thus, the law of $\tilde{C}_{0}^{(\boldsymbol{u}, \boldsymbol{v})}(\boldsymbol{y})$ is described by $\mathbb{E}_{0}$.

Lemma 2.2 (Factorisation Lemma). Fix $p \in\left[0,\|D\|_{\infty}^{-1}\right]$, a bond $(\boldsymbol{u}, \boldsymbol{v})$, a vertex $\boldsymbol{y}$, a positive integer $n$, and events $E, F$ which depend only on the status of bonds whose vertices have time variables at most $n$. Then

$$
\mathbb{E}\left(I\left[E \text { in } \tilde{C}^{(\boldsymbol{u}, \boldsymbol{v})}(\boldsymbol{y}), F \text { in } \Lambda \backslash \tilde{\boldsymbol{C}}^{(\boldsymbol{u}, \boldsymbol{v})}(\boldsymbol{y})\right]\right)=\mathbb{E}_{0}\left(I\left[E \text { in } \tilde{C}_{0}^{(\boldsymbol{u}, \boldsymbol{v})}(\boldsymbol{y})\right] \mathbb{E}_{1}\left(I\left[F \text { in } \Lambda \backslash \tilde{C}_{0}^{(\boldsymbol{u}, \boldsymbol{v})}(\boldsymbol{y})\right]\right)\right) .
$$

Moreover, when $E \subseteq\left\{\boldsymbol{u} \in \tilde{C}^{(\boldsymbol{u}, \boldsymbol{v})}(\boldsymbol{y}), \boldsymbol{v} \notin \tilde{C}^{(\boldsymbol{u}, \boldsymbol{v})}(\boldsymbol{y})\right\}$, the event on the left-hand side of (2.6) is independent of the occupation status of $(\boldsymbol{u}, \boldsymbol{v})$.

Proof. Because of our assumption on the events $E$ and $F$, we can replace the set $\tilde{C}^{(\boldsymbol{u}, \boldsymbol{v})}(\boldsymbol{y})$ in (2.6) by its restriction to vertices which are endpoints of bonds whose vertices have time variables at most $n$ (i.e., we set all other bonds to be vacant). We denote this restriction by $\tilde{C}_{n}^{(\boldsymbol{u}, \boldsymbol{v})}(\boldsymbol{y})$, and note that this is a finite set with probability 1 by (1.4). The proof proceeds by conditioning on $\tilde{C}_{n}^{(\boldsymbol{u}, \boldsymbol{v})}(\boldsymbol{y})$. We emphasise that $\tilde{C}_{n}^{(\boldsymbol{u}, \boldsymbol{v})}(\boldsymbol{y})$ is a set of vertices. Thus, $\tilde{C}_{n}^{(\boldsymbol{u}, \boldsymbol{v})}(\boldsymbol{y})=S$ does not determine the occupation status of all the bonds $b$ with both vertices in $S$. The left-hand side of (2.6) equals

$$
\sum_{S} \mathbb{P}\left(\{E \text { in } S\} \cap\{F \text { in } \Lambda \backslash S\} \mid \tilde{C}_{n}^{(\boldsymbol{u}, \boldsymbol{v})}(\boldsymbol{y})=S\right) \mathbb{P}\left(\tilde{C}_{n}^{(\boldsymbol{u}, \boldsymbol{v})}(\boldsymbol{y})=S\right),
$$

where the sum over $S$ is over finite subsets of $\Lambda$ containing $\boldsymbol{y}$.

By Definition 2.1(ii), the event $\{E$ in $S\}$ depends only on bonds with both endpoints in $S$, while the event $\{F$ in $\Lambda \backslash S\}$ depends only on bonds with both endpoints in $\Lambda \backslash S$. The latter is equivalent to saying that $\{F$ in $\Lambda \backslash S\}$ depends only on bonds that have no endpoints in $S$. Thus, by the independence of the bond variables, we obtain that

$$
\mathbb{P}\left(\{E \text { in } S\} \cap\{F \text { in } \Lambda \backslash S\} \mid \tilde{C}_{n}^{(\boldsymbol{u}, \boldsymbol{v})}(\boldsymbol{y})=S\right)=\mathbb{P}\left(E \text { in } S \mid \tilde{C}_{n}^{(\boldsymbol{u}, \boldsymbol{v})}(\boldsymbol{y})=S\right) \mathbb{P}\left(F \text { in } \Lambda \backslash S \mid \tilde{C}_{n}^{(\boldsymbol{u}, \boldsymbol{v})}(\boldsymbol{y})=S\right) .
$$

Moreover, the event $\left\{\tilde{C}_{n}^{(\boldsymbol{u}, \boldsymbol{v})}(\boldsymbol{y})=S\right\}$ depends only on bonds that have at least one endpoint in $S$. Therefore, for fixed $S$, the events $\{F$ in $\Lambda \backslash S\}$ and $\left\{\tilde{C}_{n}^{(\boldsymbol{u}, \boldsymbol{v})}(\boldsymbol{y})=S\right\}$ are independent, and hence

$$
\mathbb{P}\left(F \text { in } \Lambda \backslash S \mid \tilde{C}_{n}^{(\boldsymbol{u}, \boldsymbol{v})}(\boldsymbol{y})=S\right)=\mathbb{P}(F \text { in } \Lambda \backslash S) .
$$

Thus, we obtain

$$
\mathbb{P}\left(\{E \text { in } S\} \cap\{F \text { in } \Lambda \backslash S\} \mid \tilde{C}_{n}^{(\boldsymbol{u}, \boldsymbol{v})}(\boldsymbol{y})=S\right)=\mathbb{P}_{0}\left(E \text { in } S \mid \tilde{C}_{n}^{(\boldsymbol{u}, \boldsymbol{v})}(\boldsymbol{y})=S\right) \mathbb{P}_{1}(F \text { in } \Lambda \backslash S),
$$

where we have added subscripts to the probabilities on the right-hand side to distinguish the different expectations. We substitute (2.10) into (2.7), perform the sum over $S$, and replace $\tilde{C}_{n}$ by $\tilde{C}$, to get (2.6).

Finally, when $E \subseteq\left\{\boldsymbol{u} \in \tilde{C}^{(\boldsymbol{u}, \boldsymbol{v})}(\boldsymbol{y}), \boldsymbol{v} \notin \tilde{C}^{(\boldsymbol{u}, \boldsymbol{v})}(\boldsymbol{y})\right\}$, the event on the left-hand side of (2.6) is independent of the occupation status of the bond $(\boldsymbol{u}, \boldsymbol{v})$. For $\left\{E\right.$ in $\left.\tilde{C}^{(\boldsymbol{u}, \boldsymbol{v})}(\boldsymbol{y})\right\}$, this is because $\boldsymbol{v} \notin \tilde{C}^{(\boldsymbol{u}, \boldsymbol{v})}(\boldsymbol{y})$, and, for $\left\{F\right.$ in $\left.\Lambda \backslash \tilde{C}^{(\boldsymbol{u}, \boldsymbol{v})}(\boldsymbol{y})\right\}$, it is because $\boldsymbol{u} \in \tilde{C}^{(\boldsymbol{u}, \boldsymbol{v})}(\boldsymbol{y})$.

Although we do not need it here, we note that Lemma 2.2 also applies (both for oriented and unoriented percolation) to arbitrary events $E$ and $F$, if we replace the assumption that $E$ and $F$ are determined by bonds lying below $n$ by the assumption that $\mathbb{P}_{p}(|C(0)|=\infty)=0$.

We will refer to a bond $(\boldsymbol{u}, \boldsymbol{v})$ to which we can effectively apply Lemma 2.2 as a cutting bond. In the nested expectation on the right-hand side of (2.6), the set $\tilde{C}_{0}^{(\boldsymbol{u}, \boldsymbol{v})}(\boldsymbol{y})$ is random with respect to the outer expectation, but deterministic with respect to the inner expectation. We have added a subscript " 0 " to $\tilde{C}_{0}^{(\boldsymbol{u}, \boldsymbol{v})}(\boldsymbol{y})$ and subscripts " 0 "

\footnotetext{
1 Some versions of Lemma 2.2 published previously [5,6,14] contain non-essential errors. However, on each occasion in these papers where the Factorisation Lemma has been applied, the claimed factorisation does in fact hold.
} 
and " 1 " to the expectations on the right-hand side of (2.6) to emphasise this distinction. The inner expectation on the right-hand side effectively introduces a second percolation model on a second lattice, which is coupled to the first percolation model via the set $\tilde{C}_{0}^{(\boldsymbol{u}, \boldsymbol{v})}(\boldsymbol{y})$.

\section{The linear term}

In this section, we prove (1.28) by expanding the survival probability to linear order. In Section 3.1, we define pivotal bonds, and rewrite events dealing with pivotal bonds using Definition 2.1. In Section 3.2, we perform a first expansion step making crucial use of the Factorisation Lemma, Lemma 2.2. The first expansion is virtually identical to the expansion for the two-point function performed in [5] and [14]. In Section 3.3, we iterate this expansion step indefinitely to obtain (1.28).

\subsection{Pivotal bonds}

\section{Definition 3.1.}

(i) Given a bond configuration, we say that $\boldsymbol{x}$ is doubly connected to $\boldsymbol{y}$, written $\boldsymbol{x} \Rightarrow \boldsymbol{y}$, if there are at least two bonddisjoint paths from $\boldsymbol{x}$ to $\boldsymbol{y}$ consisting of occupied bonds. By convention, we say that $\boldsymbol{x} \Rightarrow \boldsymbol{x}$ for all $\boldsymbol{x}$. Similarly, we say that $\boldsymbol{y}$ is doubly connected to $n$, and write $\boldsymbol{y} \Rightarrow n$, if there exist $x_{1}, x_{2} \in \mathbb{Z}^{d}$ (possibly equal) and two bond-disjoint paths from $\boldsymbol{y}$ to $\left(x_{1}, n\right)$ and $\left(x_{2}, n\right)$.

(ii) Given a bond configuration, we say that a bond is pivotal for $\boldsymbol{x} \rightarrow \boldsymbol{y}$ if $\boldsymbol{x} \rightarrow \boldsymbol{y}$ in the (possibly modified) configuration in which the bond is made occupied, whereas $\boldsymbol{x}$ is not connected to $\boldsymbol{y}$ in the (possibly modified) configuration in which the bond is made vacant. Similarly, we say that a bond is pivotal for $\boldsymbol{y} \rightarrow n$ if $\boldsymbol{y} \rightarrow n$ in the (possibly modified) configuration in which the bond is made occupied, whereas $\boldsymbol{y}$ is not connected to $n$ in the (possibly modified) configuration in which the bond is made vacant.

The set of pivotal bonds for $\boldsymbol{x} \rightarrow \boldsymbol{y}$ or $\boldsymbol{y} \rightarrow n$ is ordered in time, which allows us to speak about the first pivotal bond having a certain property. We can visualise a configuration where $\mathbf{0} \rightarrow n$ as consisting of a string of sausages, the strings representing the pivotal bonds, and the sausages the parts of the cluster of $\mathbf{0}$ that are separated by the pivotal bonds. See Fig. 1 for a schematic representation of the event $\mathbf{0} \rightarrow n$ as a string of sausages.

In terms of Definitions 2.1 and 3.1, we have a characterisation of a pivotal bond for $\boldsymbol{v} \rightarrow \boldsymbol{y}$ as

$$
\left\{\left(\boldsymbol{u}^{\prime}, \boldsymbol{v}^{\prime}\right) \text { pivotal for } \boldsymbol{v} \rightarrow \boldsymbol{y}\right\}=\left\{\boldsymbol{v} \rightarrow \boldsymbol{u}^{\prime} \text { in } \tilde{\boldsymbol{C}}^{\left(\boldsymbol{u}^{\prime}, \boldsymbol{v}^{\prime}\right)}(\boldsymbol{v})\right\} \cap\left\{\boldsymbol{v}^{\prime} \rightarrow \boldsymbol{y} \text { in } \Lambda \backslash \tilde{C}^{\left(\boldsymbol{u}^{\prime}, \boldsymbol{v}^{\prime}\right)}(\boldsymbol{v})\right\} .
$$

Similarly, we have a characterisation of a pivotal bond for $\boldsymbol{v} \rightarrow n$ as

$$
\left\{\left(\boldsymbol{u}^{\prime}, \boldsymbol{v}^{\prime}\right) \text { pivotal for } \boldsymbol{v} \rightarrow n\right\}=\left\{\left\{\boldsymbol{v} \rightarrow \boldsymbol{u}^{\prime}\right\} \cap\{\boldsymbol{v} \rightarrow n\}^{c} \text { in } \tilde{C}^{\left(\boldsymbol{u}^{\prime}, \boldsymbol{v}^{\prime}\right)}(\boldsymbol{v})\right\} \cap\left\{\boldsymbol{v}^{\prime} \rightarrow n \text { in } \Lambda \backslash \tilde{C}^{\left(\boldsymbol{u}^{\prime}, \boldsymbol{v}^{\prime}\right)}(\boldsymbol{v})\right\} .
$$

The right-hand sides of (3.1), (3.2) are convenient for application of the Factorisation Lemma 2.2.

\subsection{The first expansion step}

We will successively expand the survival probability, using the notion of pivotal bonds defined in Section 3.1, the rewrite in (3.2) and Factorisation Lemma 2.2. It turns out that in the process of deriving the expansion for $\theta_{n}$, we

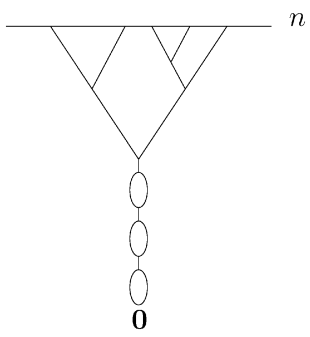

Fig. 1. Schematic representation of the event $\mathbf{0} \rightarrow n$ as a string of sausages. 
encounter an adaptation of the survival probability that we denote by the generalised survival probability. Therefore, it is most convenient to immediately expand the generalised survival probability. We start by defining the generalised survival probability. This definition will be crucial throughout the expansion.

Definition 3.2. Given a bond configuration and a set $A \subseteq \Lambda$, we say that $\boldsymbol{y}$ is connected to $\boldsymbol{x}$ through $A$, and write $\boldsymbol{y} \stackrel{A}{\rightarrow} \boldsymbol{x}$, if every occupied path connecting $\boldsymbol{y}$ to $\boldsymbol{x}$ has at least one bond with an endpoint in $A$. By convention, $\boldsymbol{x} \stackrel{A}{\rightarrow} \boldsymbol{x}$ holds if and only if $\boldsymbol{x} \in A$. Similarly, we say that $\boldsymbol{y}$ is connected to $n$ through $A$, and write $\boldsymbol{y} \stackrel{A}{\rightarrow} n$, if every occupied path connecting $\boldsymbol{y}$ to a vertex in $\mathbb{Z}^{d} \times\{n\}$ has at least one bond with an endpoint in $A$, or if $\boldsymbol{y} \in\left(\mathbb{Z}^{d} \times\{n\}\right) \cap A$.

As mentioned above it will be convenient to expand not only $\theta_{n}$, but also the generalised survival probability $\mathbb{P}(\boldsymbol{v} \stackrel{A}{\rightarrow} n)$ for a fixed vertex $\boldsymbol{v}$ and set of vertices $A$. We note that, with $\mathbf{0}=(0,0)$, we have

$$
\mathbb{P}(\mathbf{0} \stackrel{\{\mathbf{0}\}}{\longrightarrow} n)=\theta_{n} .
$$

To analyse $\mathbb{P}(\boldsymbol{v} \stackrel{A}{\rightarrow} n)$, we define the events

$$
\begin{aligned}
& E^{\prime}(\boldsymbol{v}, \boldsymbol{x} ; A)=\{\boldsymbol{v} \stackrel{A}{\rightarrow} \boldsymbol{x}\} \cap\left\{\nexists \text { pivotal bond }\left(\boldsymbol{u}^{\prime}, \boldsymbol{v}^{\prime}\right) \text { for } \boldsymbol{v} \rightarrow \boldsymbol{x} \text { such that } \boldsymbol{v} \stackrel{A}{\rightarrow} \boldsymbol{u}^{\prime}\right\}, \\
& F_{n}^{\prime}(\boldsymbol{v} ; A)=\{\boldsymbol{v} \stackrel{A}{\rightarrow} n\} \cap\left\{\nexists \text { pivotal bond }\left(\boldsymbol{u}^{\prime}, \boldsymbol{v}^{\prime}\right) \text { for } \boldsymbol{v} \rightarrow n \text { such that } \boldsymbol{v} \stackrel{A}{\rightarrow} \boldsymbol{u}^{\prime}\right\},
\end{aligned}
$$

which are depicted schematically in Fig. 2.

Given a configuration in which $\boldsymbol{v} \stackrel{A}{\rightarrow} n$, the cutting bond $\left(\boldsymbol{u}^{\prime}, \boldsymbol{v}^{\prime}\right)$ is defined to be the first occupied and pivotal bond for $\boldsymbol{v} \rightarrow n$ such that $\boldsymbol{v} \stackrel{A}{\rightarrow} \boldsymbol{u}^{\prime}$. It is possible that no such bond exists. By partitioning $\{\boldsymbol{v} \stackrel{A}{\rightarrow} n\}$ according to the location of the cutting bond (or the lack of a cutting bond), we obtain the decomposition given in the following lemma. Here and elsewhere, we write $\dot{U}$ for a disjoint union.

Lemma 3.3 (The partition). For any $v \in \Lambda, A \subseteq \Lambda, n \geqslant 0$,

$$
\{\boldsymbol{v} \stackrel{A}{\rightarrow} n\}=F_{n}^{\prime}(\boldsymbol{v} ; A) \dot{\cup} \bigcup_{\left(\boldsymbol{u}^{\prime}, \boldsymbol{v}^{\prime}\right)}^{\cdot}\left[E^{\prime}\left(\boldsymbol{v}, \boldsymbol{u}^{\prime} ; A\right) \cap\left\{\left(\boldsymbol{u}^{\prime}, \boldsymbol{v}^{\prime}\right) \text { occupied and pivotal for } \boldsymbol{v} \rightarrow n\right\}\right] .
$$

Proof. We decompose the event $\{\boldsymbol{v} \stackrel{A}{\rightarrow} n\}$ depending on whether there is a cutting bond or not. The event $F_{n}^{\prime}(\boldsymbol{v} ; A)$ is the contribution where such a cutting bond does not exist. Otherwise, let $\left(\boldsymbol{u}^{\prime}, \boldsymbol{v}^{\prime}\right)$ be the cutting bond. Then, $\left(\boldsymbol{u}^{\prime}, \boldsymbol{v}^{\prime}\right)$ is occupied and pivotal for $\boldsymbol{v} \rightarrow n$ and $\left\{\boldsymbol{v} \stackrel{A}{\rightarrow} \boldsymbol{u}^{\prime}\right\}$ holds. Moreover, there cannot be a previous pivotal bond satisfying the same requirements. The latter is equivalent to the statement that, for all $b$ that are occupied and pivotal for $\boldsymbol{v} \rightarrow \boldsymbol{u}^{\prime}$, the event $\{\boldsymbol{v} \stackrel{A}{\rightarrow} \underline{b}\}$ cannot hold. Therefore, $E^{\prime}\left(\boldsymbol{v}, \boldsymbol{u}^{\prime} ; A\right)$ holds.

Define

$$
\gamma_{n}^{(0)}(\boldsymbol{v} ; A)=\mathbb{P}\left(F_{n}^{\prime}(\boldsymbol{v} ; A)\right)
$$

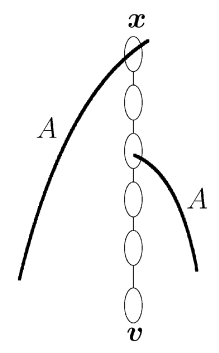

(a)

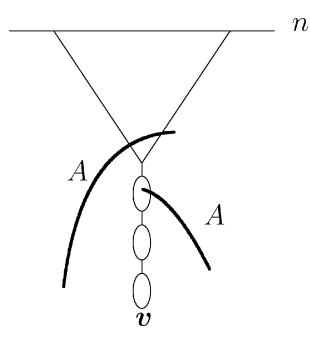

(b)

Fig. 2. (a) Schematic representation of the event $E^{\prime}(\boldsymbol{v}, \boldsymbol{x} ; A)$. The intersection of $A$ with the fourth sausage is optional, while the intersection with the sixth is required. (b) Schematic representation of the event $F_{n}^{\prime}(v ; A)$. The intersection of $A$ with the third sausage is optional, while the other intersection is required. 
Then (3.6) implies that

$$
\mathbb{P}(\boldsymbol{v} \stackrel{A}{\rightarrow} n)=\gamma_{n}^{(0)}(\boldsymbol{v} ; A)+\sum_{\left(\boldsymbol{u}^{\prime}, \boldsymbol{v}^{\prime}\right)} \mathbb{P}\left(E^{\prime}\left(\boldsymbol{v}, \boldsymbol{u}^{\prime} ; A\right) \cap\left\{\left(\boldsymbol{u}^{\prime}, \boldsymbol{v}^{\prime}\right) \text { occupied and pivotal for } \boldsymbol{v} \rightarrow n\right\}\right) .
$$

We next note that the event that $b$ is pivotal for $v \rightarrow n$ is independent of the occupation status of the bond $b$. Moreover, also $E^{\prime}\left(\boldsymbol{v}, \boldsymbol{u}^{\prime} ; A\right)$ is independent of the occupation status of the bond $\left(\boldsymbol{u}^{\prime}, \boldsymbol{v}^{\prime}\right)$, due to the orientation. Therefore, (3.8) becomes

$$
\mathbb{P}(\boldsymbol{v} \stackrel{A}{\rightarrow} n)=\sum_{\left(\boldsymbol{u}^{\prime}, \boldsymbol{v}^{\prime}\right)} J_{\boldsymbol{u}^{\prime}, \boldsymbol{v}^{\prime}} \mathbb{P}\left(E^{\prime}\left(\boldsymbol{v}, \boldsymbol{u}^{\prime} ; A\right) \cap\left\{\left(\boldsymbol{u}^{\prime}, \boldsymbol{v}^{\prime}\right) \text { pivotal for } \boldsymbol{v} \rightarrow n\right\}\right)+\gamma_{n}^{(0)}(\boldsymbol{v} ; A),
$$

where we make the abbreviation

$$
J_{(u, m),(v, n)}=p D(v-u) \delta_{n, m+1} .
$$

We note that, by the orientation of the bonds, the event $E^{\prime}\left(\boldsymbol{v}, \boldsymbol{u}^{\prime} ; A\right)$ is independent of the bonds above $\boldsymbol{u}^{\prime}$, so that

$$
E^{\prime}\left(\boldsymbol{v}, \boldsymbol{u}^{\prime} ; A\right)=\left\{E^{\prime}\left(\boldsymbol{v}, \boldsymbol{u}^{\prime} ; A\right) \text { in } \tilde{C}^{\left(\boldsymbol{u}^{\prime}, \boldsymbol{v}^{\prime}\right)}(\boldsymbol{v})\right\} .
$$

We use (3.2), together with (3.11) and (2.3), to rewrite the event on the right-hand side of (3.9) as

$$
\begin{aligned}
& E^{\prime}\left(\boldsymbol{v}, \boldsymbol{u}^{\prime} ; A\right) \cap\left\{\left(\boldsymbol{u}^{\prime}, \boldsymbol{v}^{\prime}\right) \text { pivotal for } \boldsymbol{v} \rightarrow n\right\} \\
& \quad=\left\{\left\{E^{\prime}\left(\boldsymbol{v}, \boldsymbol{u}^{\prime} ; A\right) \cap\{\boldsymbol{v} \rightarrow n\}^{c}\right\} \text { in } \tilde{C}^{\left(\boldsymbol{u}^{\prime}, \boldsymbol{v}^{\prime}\right)}(\boldsymbol{v})\right\} \cap\left\{\boldsymbol{v}^{\prime} \rightarrow n \text { in } \Lambda \backslash \tilde{C}^{\left(\boldsymbol{u}^{\prime}, \boldsymbol{v}^{\prime}\right)}(\boldsymbol{v})\right\} .
\end{aligned}
$$

Using Lemma 2.2, we obtain from (3.9) and (3.12) the important rewrite

$$
\begin{aligned}
\mathbb{P}(\boldsymbol{v} \stackrel{A}{\rightarrow} n)= & \sum_{\left(\boldsymbol{u}^{\prime}, \boldsymbol{v}^{\prime}\right)} J_{\boldsymbol{u}^{\prime}, \boldsymbol{v}^{\prime}} \mathbb{E}_{0}\left(I\left[E^{\prime}\left(\boldsymbol{v}, \boldsymbol{u}^{\prime} ; A\right) \cap\{\boldsymbol{v} \rightarrow n\}^{c} \text { in } \tilde{C}_{0}^{\left(\boldsymbol{u}^{\prime}, \boldsymbol{v}^{\prime}\right)}(\boldsymbol{v})\right]\right. \\
& \left.\times \mathbb{P}_{1}\left(\boldsymbol{v}^{\prime} \rightarrow n \text { in } \Lambda \backslash \tilde{C}_{0}^{\left(\boldsymbol{u}^{\prime}, \boldsymbol{v}^{\prime}\right)}(\boldsymbol{v})\right)\right)+\gamma_{n}^{(0)}(\boldsymbol{v} ; A) .
\end{aligned}
$$

We next use the inclusion-exclusion relation

$$
I\left[\{\boldsymbol{v} \rightarrow n\}^{c}\right]=1-I[\{\boldsymbol{v} \rightarrow n\}],
$$

which brings us to

$$
\mathbb{P}(\boldsymbol{v} \stackrel{A}{\rightarrow} n)=\sum_{\left(\boldsymbol{u}^{\prime}, \boldsymbol{v}^{\prime}\right)} J_{\boldsymbol{u}^{\prime}, \boldsymbol{v}^{\prime}} \mathbb{E}_{0}\left(I\left[E^{\prime}\left(\boldsymbol{v}, \boldsymbol{u}^{\prime} ; A\right)\right] \mathbb{P}_{1}\left(\boldsymbol{v}^{\prime} \rightarrow n \text { in } \Lambda \backslash \tilde{C}_{0}^{\left(\boldsymbol{u}^{\prime}, \boldsymbol{v}^{\prime}\right)}(\boldsymbol{v})\right)\right)+\gamma_{n}^{(0)}(\boldsymbol{v} ; A)-\rho_{n}^{(0)}(\boldsymbol{v} ; A),
$$

where

$$
\rho_{n}^{(0)}(\boldsymbol{v} ; A)=\sum_{\left(\boldsymbol{u}^{\prime}, \boldsymbol{v}^{\prime}\right)} J_{\boldsymbol{u}^{\prime}, \boldsymbol{v}^{\prime}} \mathbb{E}_{0}\left(I\left[E^{\prime}\left(\boldsymbol{v}, \boldsymbol{u}^{\prime} ; A\right) \cap\{\boldsymbol{v} \rightarrow n\} \text { in } \tilde{C}_{0}^{\left(\boldsymbol{u}^{\prime}, \boldsymbol{v}^{\prime}\right)}(\boldsymbol{v})\right] \mathbb{P}_{1}\left(\boldsymbol{v}^{\prime} \rightarrow n \text { in } \Lambda \backslash \tilde{C}_{0}^{\left(\boldsymbol{u}^{\prime}, \boldsymbol{v}^{\prime}\right)}(\boldsymbol{v})\right)\right) .
$$

We have omitted "in $\tilde{C}_{0}^{\left(u^{\prime}, v^{\prime}\right)}(v)$ " in the sum in (3.15), which is possible due to (3.11).

Finally, let $\theta_{n}(\boldsymbol{v})=\mathbb{P}(\boldsymbol{v} \rightarrow n)$. Then, for every $A \subseteq \Lambda$,

$$
\mathbb{P}(\boldsymbol{v} \rightarrow n \text { in } \Lambda \backslash A)=\theta_{n}(\boldsymbol{v})-\mathbb{P}(\boldsymbol{v} \stackrel{A}{\rightarrow} n) .
$$

If we define

$$
\pi^{(0)}(\boldsymbol{v}, \boldsymbol{x} ; A)=\mathbb{P}\left(E^{\prime}(\boldsymbol{v}, \boldsymbol{x} ; A)\right),
$$

then (3.15) and (3.17) yield the identity

$$
\begin{aligned}
\mathbb{P}(\boldsymbol{v} \stackrel{A}{\rightarrow} n)= & \gamma_{n}^{(0)}(\boldsymbol{v} ; A)-\rho_{n}^{(0)}(\boldsymbol{v} ; A)+\sum_{\left(\boldsymbol{u}^{\prime}, \boldsymbol{v}^{\prime}\right)} J_{\boldsymbol{u}^{\prime}, \boldsymbol{v}^{\prime}} \pi^{(0)}\left(\boldsymbol{v}, \boldsymbol{u}^{\prime} ; A\right) \theta_{n}\left(\boldsymbol{v}^{\prime}\right) \\
& -\sum_{\left(\boldsymbol{u}^{\prime}, \boldsymbol{v}^{\prime}\right)} J_{\boldsymbol{u}^{\prime}, \boldsymbol{v}^{\prime}} \mathbb{E}_{0}\left(I\left[E^{\prime}\left(\boldsymbol{v}, \boldsymbol{u}^{\prime} ; A\right)\right] \mathbb{P}_{1}\left(\boldsymbol{v}^{\prime} \stackrel{\tilde{C}_{0}^{\left(\boldsymbol{u}^{\prime}, \boldsymbol{v}^{\prime}\right)}(\boldsymbol{v})}{\longrightarrow} n\right)\right) .
\end{aligned}
$$

This completes the first expansion step. 


\subsection{Iteration}

In the right-hand side of (3.19), we again see a term of the form $\mathbb{P}_{1}(\boldsymbol{v} \stackrel{A}{\rightarrow} n)$, but now with $A=\tilde{C}_{0}^{\left(\boldsymbol{u}^{\prime}, \boldsymbol{v}^{\prime}\right)}(\boldsymbol{v})$ and with $\boldsymbol{v}$ replaced by $\boldsymbol{v}^{\prime}$. Thus, we can iterate (3.19). To write down this iteration, we first define, for any random variable $X$,

$$
M_{\boldsymbol{v}, \boldsymbol{y} ; A}^{(1)}(X)=\mathbb{E}_{0}\left(I\left[E^{\prime}(\boldsymbol{v}, \boldsymbol{y} ; A)\right] X\right) .
$$

For $N \geqslant 2$, we define $M_{\boldsymbol{v}, \boldsymbol{y} ; A}^{(N)}(X)$ recursively by

$$
M_{\boldsymbol{v}, \boldsymbol{y} ; A}^{(N)}(X)=\sum_{\left(\boldsymbol{u}_{N-2}, \boldsymbol{v}_{N-2}\right)} J_{\boldsymbol{u}_{N-2}, \boldsymbol{v}_{N-2}} M_{\boldsymbol{v}, \boldsymbol{u}_{N-2} ; A}^{(N-1)}\left(M_{\boldsymbol{v}_{N-2}, \boldsymbol{y} ; \tilde{C}_{N-2}}^{(1)}(X)\right),
$$

where, for $j \geqslant 0$, we make the abbreviation $\tilde{C}_{j}=\tilde{C}_{j}^{\left(\boldsymbol{u}_{j}, \boldsymbol{v}_{j}\right)}\left(\boldsymbol{v}_{j-1}\right)$, with $\boldsymbol{v}_{-1}=\boldsymbol{v}$, and where the expectation occurring in $M_{\boldsymbol{v}_{N-2}, \boldsymbol{y} ; \tilde{C}_{N-2}}^{(1)}(X)$ is labelled $N-1$. For example, when $N=2, X=1, \boldsymbol{v}=\mathbf{0}$, and $A=\{\mathbf{0}\}$,

$$
M_{\mathbf{0}, \boldsymbol{y} ;\{\boldsymbol{0}\}}^{(2)}(1)=\sum_{\left(\boldsymbol{u}_{0}, \boldsymbol{v}_{0}\right)} J_{\boldsymbol{u}_{0}, \boldsymbol{v}_{0}} \mathbb{E}_{0}\left(I\left[E^{\prime}\left(\mathbf{0}, \boldsymbol{u}_{0} ;\{\mathbf{0}\}\right)\right] \mathbb{E}_{1}\left(I\left[E^{\prime}\left(\boldsymbol{v}_{0}, \boldsymbol{y} ; \tilde{C}_{0}\right)\right]\right)\right) .
$$

Note that, by (3.4),

$$
E^{\prime}\left(\mathbf{0}, \boldsymbol{u}_{0} ;\{\mathbf{0}\}\right)=\left\{\mathbf{0} \Longrightarrow \boldsymbol{u}_{0}\right\} .
$$

According to [14, Eq. (3.25)], the coefficients of the lace expansion for the two-point function in (1.13) are given in terms of the above notation by

$$
\pi_{m}=\sum_{N=0}^{\infty}(-1)^{N} \pi_{m}^{(N)},
$$

with, for $N \geqslant 0$,

$$
\pi_{m}^{(N)}=\sum_{y \in \mathbb{Z}^{d}} \pi_{m}^{(N)}(y), \quad \pi_{m}^{(N)}(y)=M_{\mathbf{0},(y, m) ;\{0\}}^{(N+1)}(1) .
$$

Note that here we adopt the convention that $\pi_{m}^{(0)}(y)=\mathbb{P}(\mathbf{0} \Rightarrow(y, m))$, rather than the convention $\pi_{m}^{(0)}(y)=$ $\mathbb{P}(\mathbf{0} \Rightarrow(y, m))-\delta_{0, m} \delta_{0, y}$ used in [14].

We define, for $N \geqslant 1$,

$$
\gamma_{n}^{(N)}(\boldsymbol{v} ; A)=\sum_{\left(\boldsymbol{u}_{N-1}, \boldsymbol{v}_{N-1}\right)} J_{\boldsymbol{u}_{N-1}, \boldsymbol{v}_{N-1}} M_{\boldsymbol{v}, \boldsymbol{u}_{N-1} ; A}^{(N)}\left(\gamma_{n}^{(0)}\left(\boldsymbol{v}_{N-1} ; \tilde{C}_{N-1}\right)\right)
$$

with $\gamma_{n}^{(0)}(\boldsymbol{v} ; A)$ defined in (3.7), and, for $N \geqslant 1$,

$$
\begin{aligned}
& \pi^{(N)}(\boldsymbol{v}, \boldsymbol{x} ; A)=M_{\boldsymbol{v}, \boldsymbol{x} ; A}^{(N+1)}(1), \\
& \rho_{n}^{(N)}(\boldsymbol{v} ; A)=\sum_{\left(\boldsymbol{u}_{N-1}, \boldsymbol{v}_{N-1}\right)} J_{\boldsymbol{u}_{N-1}, \boldsymbol{v}_{N-1}} M_{\boldsymbol{v}, \boldsymbol{u}_{N-1} ; A}^{(N)}\left(\rho_{n}^{(0)}\left(\boldsymbol{v}_{N-1} ; \tilde{C}_{N-1}\right)\right),
\end{aligned}
$$

with $\pi^{(0)}(\boldsymbol{v}, \boldsymbol{x} ; A)$ and $\rho_{n}^{(0)}(\boldsymbol{v} ; A)$ defined in (3.18) and (3.16). We let

$$
\chi_{n}^{(N)}(\boldsymbol{v} ; A)=\gamma_{n}^{(N)}(\boldsymbol{v} ; A)-\rho_{n}^{(N)}(\boldsymbol{v} ; A) .
$$

We omit the superscript " $(N)$ " to denote the alternating sum over $N$, e.g.,

$$
\begin{aligned}
& \pi(\boldsymbol{v}, \boldsymbol{x} ; A)=\sum_{N=0}^{\infty}(-1)^{N} \pi^{(N)}(\boldsymbol{v}, \boldsymbol{x} ; A), \\
& \chi_{n}(\boldsymbol{v} ; A)=\sum_{N=0}^{\infty}(-1)^{N} \chi_{n}^{(N)}(\boldsymbol{v} ; A) .
\end{aligned}
$$



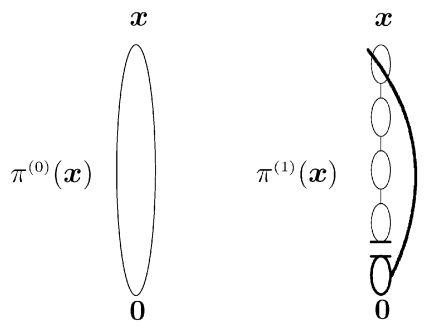

Fig. 3. Schematic representations of $\pi^{(N)}(\boldsymbol{x})$ for $N=0,1$. For $\pi^{(1)}(\boldsymbol{x})$, the bold and thin lines correspond to the different expectations.
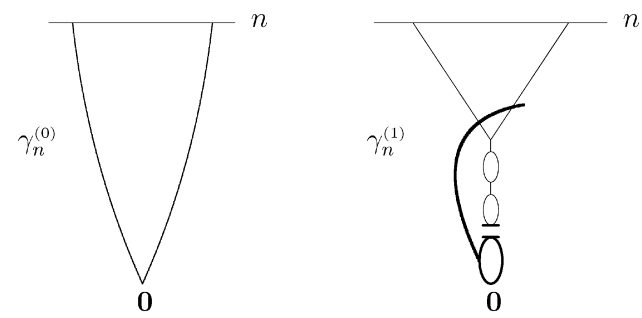

Fig. 4. Schematic representation of $\gamma_{n}^{(N)}$ for $N=0,1$. For $\gamma_{n}^{(1)}$, the bold and thin lines correspond to different expectations.
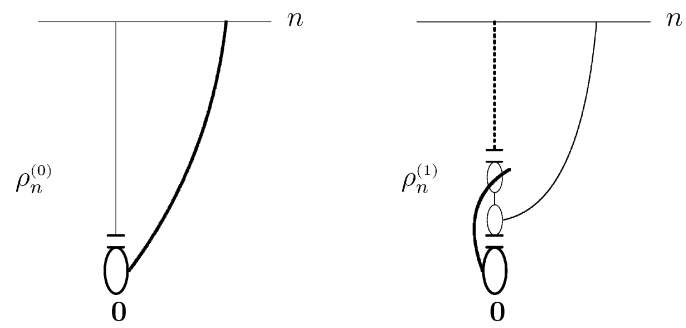

Fig. 5. Schematic representation of $\rho_{n}^{(N)}$ for $N=0,1$. For $\rho_{n}^{(0)}$ there are two distinct expectations, and for $\rho_{n}^{(1)}$ there are three.

In the special case $\boldsymbol{v}=\mathbf{0}$ and $A=\{\boldsymbol{0}\}$, we omit the variables $\boldsymbol{v}$ and $A$ and write

$$
\pi(\boldsymbol{x})=\pi(\mathbf{0}, \boldsymbol{x} ;\{\mathbf{0}\}), \quad \chi_{n}=\chi_{n}(\mathbf{0} ;\{\mathbf{0}\}), \quad \gamma_{n}=\gamma_{n}(\mathbf{0} ;\{\mathbf{0}\}), \quad \rho_{n}=\rho_{n}(\mathbf{0} ;\{\mathbf{0}\}),
$$

and similarly for $\pi^{(N)}(\boldsymbol{x}), \chi_{n}^{(N)}, \gamma_{n}^{(N)}$ and $\rho_{n}^{(N)}$. In particular, $\gamma_{n}^{(0)}=\mathbb{P}(\mathbf{0} \Rightarrow n)$. A schematic representation of $\pi^{(N)}(\boldsymbol{x})$ for $N=0,1$ is depicted in Fig. 3, and schematic representations of $\gamma_{n}^{(N)}$ and $\rho_{n}^{(N)}$ for $N=0,1$ are depicted in Figs. 4 and 5 .

The result of the first expansion is given in the following proposition. Recall that $\theta_{n}(\boldsymbol{v})$ was defined above (3.17).

Proposition 3.4 (The linear term). For all $v \in \Lambda, A \subseteq \Lambda, n \geqslant 1$,

$$
\mathbb{P}(\boldsymbol{v} \stackrel{A}{\rightarrow} n)=\sum_{\left(\boldsymbol{u}^{\prime}, \boldsymbol{v}^{\prime}\right)} \pi\left(\boldsymbol{v}, \boldsymbol{u}^{\prime} ; A\right) J_{\boldsymbol{u}^{\prime}, \boldsymbol{v}^{\prime}} \theta_{n}\left(\boldsymbol{v}^{\prime}\right)+\chi_{n}(\boldsymbol{v} ; A) .
$$

Proof. The identity (3.19) can be rewritten, using (3.20), (3.27), and (3.29), as

$$
\mathbb{P}(\boldsymbol{v} \stackrel{A}{\rightarrow} n)=\sum_{\left(\boldsymbol{u}_{0}, \boldsymbol{v}_{0}\right)} J_{\boldsymbol{u}_{0}, \boldsymbol{v}_{0}} M_{\boldsymbol{v}, \boldsymbol{u}_{0} ; A}^{(1)}(1) \theta_{n}\left(\boldsymbol{v}_{0}\right)+\chi_{n}^{(0)}(\boldsymbol{v} ; A)-\sum_{\left(\boldsymbol{u}_{0}, \boldsymbol{v}_{0}\right)} J_{\boldsymbol{u}_{0}, \boldsymbol{v}_{0}} M_{\boldsymbol{v}, \boldsymbol{u}_{0} ; A}^{(1)}\left(\mathbb{P}_{1}\left(\boldsymbol{v}_{0} \stackrel{\tilde{C}_{0}}{\longrightarrow} n\right)\right) .
$$

Recalling (3.27), we see that the first line on the right-hand side of (3.34) is equal to the $N=0$ contribution to the right-hand side of (3.33). We will iterate (3.34) to obtain (3.33). For this, it is useful to note that a shift of indices in (3.21) gives

$$
\sum_{\left(\boldsymbol{u}_{N-1}, \boldsymbol{v}_{N-1}\right)} J_{\boldsymbol{u}_{N-1}, \boldsymbol{v}_{N-1}} M_{\boldsymbol{v}, \boldsymbol{u}_{N-1} ; A}^{(N)}\left(M_{\boldsymbol{v}_{N-1}, \boldsymbol{u}_{N} ; \tilde{C}_{N-1}}^{(1)}(X)\right)=M_{\boldsymbol{v}, \boldsymbol{u}_{N} ; A}^{(N+1)}(X) .
$$


For $N \geqslant 1$, it follows from (3.34), (3.35), together with (3.26)-(3.29) and the linearity of $X \mapsto M_{\boldsymbol{v}, \boldsymbol{u}_{N-1} ; A}^{(N)}(X)$, that

$$
\begin{aligned}
& \sum_{\left(\boldsymbol{u}_{N-1}, \boldsymbol{v}_{N-1}\right)} J_{\boldsymbol{u}_{N-1}, \boldsymbol{v}_{N-1}} M_{\boldsymbol{v}, \boldsymbol{u}_{N-1} ; A}^{(N)}\left(\mathbb{P}_{N}\left(\boldsymbol{v}_{N-1} \stackrel{\tilde{C}_{N-1}}{\longrightarrow} n\right)\right) \\
& =\sum_{\left(\boldsymbol{u}_{N}, \boldsymbol{v}_{N}\right)} J_{\boldsymbol{u}_{N}, \boldsymbol{v}_{N}} \pi_{n}^{(N)}\left(\boldsymbol{v}, \boldsymbol{u}_{N} ; A\right) \theta_{n}\left(\boldsymbol{v}_{N}\right)+\chi_{n}^{(N)}(\boldsymbol{v} ; A)-\sum_{\left(\boldsymbol{u}_{N}, \boldsymbol{v}_{N}\right)} J_{\boldsymbol{u}_{N}, \boldsymbol{v}_{N}} M_{\boldsymbol{v}, \boldsymbol{u}_{N} ; A}^{(N+1)}\left(\mathbb{P}_{N+1}\left(\boldsymbol{v}_{N} \stackrel{\tilde{C}_{N}}{\longrightarrow} n\right)\right) .
\end{aligned}
$$

We use (3.36) in (3.34) repeatedly until the last term vanishes. This must happen before $N=n+1$, because the time variable of $\boldsymbol{v}_{N}$ is strictly larger than the time variable of $\boldsymbol{v}_{N-1}$, and the last term in (3.36) is zero when the time variable of $\boldsymbol{v}_{N}$ exceeds $n$.

We complete the proof of (1.28) using Proposition 3.4. According to (3.25) and (3.27), $\pi(y)$ is equal to the coefficient $\pi_{m}(y)$ of the lace expansion for the two-point function, where $\boldsymbol{y}=(y, m)$. We use the notation $\pi_{m}(y)$ and $\pi_{m}=\sum_{y} \pi_{m}(y)$ when we wish to emphasise the role of the time variable. Since $\theta_{n}(y, m)=\theta_{n-m}$ for every $y \in \mathbb{Z}^{d}$, and since $\sum_{\boldsymbol{v}^{\prime}} J_{\boldsymbol{u}^{\prime}, \boldsymbol{v}^{\prime}}=p$ by (3.10), (3.33) reduces in this special case to

$$
\theta_{n}=\sum_{m=0}^{n-1} \pi_{m} p \theta_{n-1-m}+\chi_{n} .
$$

This proves (1.28), and we have extracted the linear term in the expansion for $\theta_{n}$.

Finally, for future reference, we prove the recursion relation

$$
M_{\boldsymbol{v}, \boldsymbol{y} ; A}^{(N+M)}(X)=\sum_{\left(\boldsymbol{u}_{N-1}, \boldsymbol{v}_{N-1}\right)} J_{\boldsymbol{u}_{N-1}, \boldsymbol{v}_{N-1}} M_{\boldsymbol{v}, \boldsymbol{u}_{N-1} ; A}^{(N)}\left(M_{\boldsymbol{v}_{N-1}, \boldsymbol{y} ; \tilde{C}_{N-1}}^{(M)}(X)\right),
$$

valid for $M, N \geqslant 1$. The proof is by induction on $M$. We first note that (3.38) holds for all $N \geqslant 1$ when $M=1$, since in this case it is identical to (3.35). We assume as induction hypothesis that

$$
M_{\boldsymbol{v}, \boldsymbol{u}_{N} ; A}^{(N+M-1)}(Y)=\sum_{\left(\boldsymbol{u}_{N-1}, \boldsymbol{v}_{N-1}\right)} J_{\boldsymbol{u}_{N-1}, \boldsymbol{v}_{N-1}} M_{\boldsymbol{v}, \boldsymbol{u}_{N-1} ; A}^{(N)}\left(M_{\boldsymbol{v}_{N-1}, \boldsymbol{u}_{N} ; \tilde{C}_{N-1}}^{(M-1)}(Y)\right),
$$

holds for all $N \geqslant 1$. To advance the induction, we substitute

$$
Y=\sum_{\left(\boldsymbol{u}_{N}, \boldsymbol{v}_{N}\right)} J_{\boldsymbol{u}_{N}, \boldsymbol{v}_{N}} M_{\boldsymbol{v}_{N}, \boldsymbol{y} ; \tilde{C}_{N}}^{(X)}(X)
$$

into (3.39). By (3.35), the left-hand side equals $M_{v, y ; A}^{(N+M)}(X)$, while the right-hand side equals the right-hand side of (3.38). This advances the induction hypothesis, and proves (3.38).

\section{The quadratic term: The first expansion for $\chi_{n}$}

In (3.37), we have established the identity

$$
\theta_{n}=\sum_{m=0}^{n-1} \pi_{m} p \theta_{n-1-m}+\chi_{n} .
$$

To prove the identity (1.17) of Theorem 1.1(i), we will show that

$$
\chi_{n}^{(N)}=-\sum_{m_{1}=1}^{\lfloor n / 2\rfloor} \sum_{m_{2}=m_{1}}^{n} \phi_{m_{1}, m_{2}}^{(N)} \theta_{n-m_{1}} \theta_{n-m_{2}}+e_{n}^{(N)},
$$

where $\phi_{m_{1}, m_{2}}^{(N)}$ are certain expansion coefficients, and $e_{n}^{(N)}$ is an error term. The desired result (1.17) then follows from (4.1), (4.2), with

$$
e_{n}=\sum_{N=0}^{\infty}(-1)^{N} e_{n}^{(N)}, \quad \phi_{m_{1}, m_{2}}=\sum_{N=0}^{\infty}(-1)^{N} \phi_{m_{1}, m_{2}}^{(N)} .
$$


In this section, we will go part way to proving (4.2), by showing that

$$
\chi_{n}^{(N)}=-\sum_{m_{1}=1}^{\lfloor n / 2\rfloor} \kappa_{m_{1}, n}^{(N)} \theta_{n-m_{1}}+e_{n}^{(N)}(1)+e_{n}^{(N)}(2)+e_{n}^{(N)}(3) .
$$

The coefficients $\kappa_{m_{1}, n}^{(N)}$ and the error terms $e_{n}^{(N)}(1), e_{n}^{(N)}(2), e_{n}^{(N)}(3)$ are defined in Section 4.2 below. The proof of (4.2) will then be completed in Section 5 , via an expansion for $\kappa_{m_{1}, n}^{(N)}$.

Recall from (3.29) that $\chi_{n}^{(N)}=\gamma_{n}^{(N)}-\rho_{n}^{(N)}$, where $\gamma_{n}^{(N)}$ and $\rho_{n}^{(N)}$ are defined in terms of $\gamma_{n}^{(0)}$ and $\rho_{n}^{(0)}$ in (3.26) and (3.28). We begin in Section 4.1 with an analysis of $\rho_{n}^{(N)}$, and continue in Section 4.2 with $\gamma_{n}^{(N)}$. Section 4.3 contains the proof of a key proposition involving an important set of bonds $\mathcal{P}_{A}$ introduced in Section 4.2. Finally, in Section 4.4, we prepare for an analysis of error terms.

\subsection{The first expansion for $\rho_{n}$}

For $A, B \subseteq \Lambda$, we define

$$
\kappa_{n}^{(0)}(\boldsymbol{v} ; A, B)=\sum_{\left(\boldsymbol{u}_{0}, \boldsymbol{v}_{0}\right)} J_{\boldsymbol{u}_{0}, \boldsymbol{v}_{0}} \mathbb{E}_{0}\left(I\left[E^{\prime}\left(\boldsymbol{v}, \boldsymbol{u}_{0} ; B\right) \cap\{\boldsymbol{v} \stackrel{A}{\rightarrow} n\} \text { in } \tilde{C}_{0}\right]\left(\theta_{n}\left(\boldsymbol{v}_{0}\right)-\mathbb{P}_{1}\left(\boldsymbol{v}_{0} \stackrel{\tilde{C}_{0}}{\longrightarrow} n\right)\right)\right),
$$

where as usual $\tilde{C}_{0}=\tilde{C}_{0}^{\left(\boldsymbol{u}_{0}, \boldsymbol{v}_{0}\right)}(\boldsymbol{v})$. By (3.16), (3.17), and the facts that $\{\boldsymbol{v} \stackrel{\{\boldsymbol{v}\}}{\longrightarrow} n\}=\{\boldsymbol{v} \rightarrow n\}$ and $\left\{\boldsymbol{v}^{\prime} \notin \tilde{C}_{0}^{\left(\boldsymbol{u}^{\prime}, \boldsymbol{v}^{\prime}\right)}(\boldsymbol{v})\right\}=$ $\left\{\left\{\boldsymbol{v}^{\prime} \notin \tilde{C}_{0}^{\left(\boldsymbol{u}^{\prime}, \boldsymbol{v}^{\prime}\right)}(\boldsymbol{v})\right\}\right.$ in $\left.\tilde{C}_{0}^{\left(\boldsymbol{u}^{\prime}, \boldsymbol{v}^{\prime}\right)}(\boldsymbol{v})\right\}$, we have

$$
\rho_{n}^{(0)}(\boldsymbol{v} ; A)=\kappa_{n}^{(0)}(\boldsymbol{v} ;\{\boldsymbol{v}\}, A) .
$$

We write $m_{v}$ for the time coordinate of a vertex $\boldsymbol{v}$, and define

$$
\begin{aligned}
& \Pi_{m}(\boldsymbol{v} ; A)=\delta_{m, m_{\boldsymbol{v}}}-p \sum_{y \in \mathbb{Z}^{d}} \pi(\boldsymbol{v},(y, m-1) ; A), \\
& \kappa_{m, n}^{(0)}(\boldsymbol{v} ; A, B)=\sum_{\left(\boldsymbol{u}_{0}, \boldsymbol{v}_{0}\right)} J_{\boldsymbol{u}_{0}, \boldsymbol{v}_{0}} \mathbb{E}_{0}\left(I\left[E^{\prime}\left(\boldsymbol{v}, \boldsymbol{u}_{0} ; B\right) \cap\{\boldsymbol{v} \stackrel{A}{\rightarrow} n\} \text { in } \tilde{C}_{0}\right] \Pi_{m}\left(\boldsymbol{v}_{0} ; \tilde{C}_{0}\right)\right), \\
& e_{n}^{(0)}(\boldsymbol{v} ; A, B)=-\sum_{\left(\boldsymbol{u}_{0}, \boldsymbol{v}_{0}\right)} J_{\boldsymbol{u}_{0}, \boldsymbol{v}_{0}} \mathbb{E}_{0}\left(I\left[E^{\prime}\left(\boldsymbol{v}, \boldsymbol{u}_{0} ; B\right) \cap\{\boldsymbol{v} \stackrel{A}{\rightarrow} n\} \text { in } \tilde{C}_{0}\right] \chi_{n}\left(\boldsymbol{v}_{0} ; \tilde{C}_{0}\right)\right) .
\end{aligned}
$$

Lemma 4.1. For $n \geqslant 1, v \in \Lambda$ and $A, B \subseteq \Lambda$,

$$
\kappa_{n}^{(0)}(\boldsymbol{v} ; A, B)=\sum_{m_{1}=1}^{n} \kappa_{m_{1}, n}^{(0)}(\boldsymbol{v} ; A, B) \theta_{n-m_{1}}+e_{n}^{(0)}(\boldsymbol{v} ; A, B) .
$$

Proof. We use Proposition 3.4 to extract one factor $\theta_{n-m_{1}}$ from the factor $\theta_{n}\left(\boldsymbol{v}_{0}\right)-\mathbb{P}_{1}\left(\boldsymbol{v}_{0} \stackrel{\tilde{C}_{0}}{\longrightarrow} n\right)$ in $\kappa_{n}^{(0)}(\boldsymbol{v} ; A, B)$. Explicitly,

$$
\begin{aligned}
\theta_{n}\left(\boldsymbol{v}_{0}\right)-\mathbb{P}_{1}\left(\boldsymbol{v}_{0} \stackrel{\tilde{C}_{0}}{\longrightarrow} n\right) & =\theta_{n}\left(\boldsymbol{v}_{0}\right)-\sum_{\left(\boldsymbol{u}_{1}, \boldsymbol{v}_{1}\right)} J_{\boldsymbol{u}_{1}, \boldsymbol{v}_{1}} \pi\left(\boldsymbol{v}_{0}, \boldsymbol{u}_{1} ; \tilde{C}_{0}\right) \theta_{n}\left(\boldsymbol{v}_{1}\right)-\chi_{n}\left(\boldsymbol{v}_{0} ; \tilde{C}_{0}\right) \\
& =\sum_{m_{1}} \Pi_{m_{1}}\left(\boldsymbol{v}_{0} ; \tilde{C}_{0}\right) \theta_{n-m_{1}}-\chi_{n}\left(\boldsymbol{v}_{0} ; \tilde{C}_{0}\right),
\end{aligned}
$$

using (4.7) in the second equality. Substitution into (4.5) gives (4.10).

We use the abbreviations

$$
\kappa_{m, n}^{(0)}(A)=\kappa_{m, n}^{(0)}(\mathbf{0} ; A,\{\mathbf{0}\}), \quad e_{n}^{(0)}(A)=e_{n}^{(0)}(\mathbf{0} ; A,\{\mathbf{0}\}),
$$

and, for $N \geqslant 1$, we define 


$$
\begin{aligned}
& \kappa_{m, n}^{(N)}(A)=\sum_{\left(\boldsymbol{u}_{N-1}, \boldsymbol{v}_{N-1}\right)} J_{\boldsymbol{u}_{N-1}, \boldsymbol{v}_{N-1}} M_{\mathbf{0}, \boldsymbol{u}_{N-1} ;\{\mathbf{0}\}}^{(N)}\left(\kappa_{m, n}^{(0)}\left(\boldsymbol{v}_{N-1} ; A, \tilde{C}_{N-1}\right)\right), \\
& e_{n}^{(N)}(A)=\sum_{\left(\boldsymbol{u}_{N-1}, \boldsymbol{v}_{N-1}\right)} J_{\boldsymbol{u}_{N-1}, \boldsymbol{v}_{N-1}} M_{\mathbf{0}, \boldsymbol{u}_{N-1} ;\{\mathbf{0}\}}^{(N)}\left(e_{n}^{(0)}\left(\boldsymbol{v}_{N-1} ; A, \tilde{C}_{N-1}\right)\right) .
\end{aligned}
$$

An abuse of notation: It will be convenient in what follows to make an abuse of notation in which we write, e.g., $\kappa_{m_{1}, n}^{(N)}\left(\left\{\boldsymbol{v}_{N-1}\right\}\right)$ to denote the result of setting $A=\left\{\boldsymbol{v}_{N-1}\right\}$ in (4.13). The variable $\boldsymbol{v}_{N-1}$ is the summation index, so that $\kappa_{m_{1}, n}^{(N)}\left(\left\{\boldsymbol{v}_{N-1}\right\}\right)$ does not actually depend on $\boldsymbol{v}_{N-1}$. Also, we will use the convention

$$
\boldsymbol{v}_{-1}=\mathbf{0}, \quad \tilde{C}_{-1}=\{\mathbf{0}\} .
$$

With the above abuse of notation, the following proposition gives the first expansion for $\rho_{n}^{(N)}$.

Proposition 4.2 (The first expansion for $\rho_{n}$ ). For $n \geqslant 1$ and $N \geqslant 0$,

$$
\rho_{n}^{(N)}=\sum_{m_{1}=1}^{n} \kappa_{m_{1}, n}^{(N)}\left(\left\{\boldsymbol{v}_{N-1}\right\}\right) \theta_{n-m_{1}}+e_{n}^{(N)}\left(\left\{\boldsymbol{v}_{N-1}\right\}\right) .
$$

Proof. By (4.6) and Lemma 4.1, we obtain

$$
\rho_{n}^{(0)}(\boldsymbol{v} ; A)=\sum_{m_{1}=1}^{n} \kappa_{m_{1}, n}^{(0)}(\boldsymbol{v} ;\{\boldsymbol{v}\}, A) \theta_{n-m_{1}}+e_{n}^{(0)}(\boldsymbol{v} ;\{\boldsymbol{v}\}, A) .
$$

The identity (4.16) then follows by substitution of (4.17) into (3.28), using (4.13), (4.14) with the abuse of notation.

For $N \geqslant 1$, we note for future reference that

$$
\begin{aligned}
& \kappa_{m, n}^{(N)}(A)=\sum_{\left(\boldsymbol{u}_{N}, \boldsymbol{v}_{N}\right)} J_{\boldsymbol{u}_{N}, \boldsymbol{v}_{N}} M_{\mathbf{0}, \boldsymbol{u}_{N} ;\{\mathbf{0}\}}^{(N+1)}\left(I\left[\boldsymbol{v}_{N-1} \stackrel{A}{\rightarrow} n \text { in } \tilde{C}_{N}\right] \Pi_{m}\left(\boldsymbol{v}_{N} ; \tilde{C}_{N}\right)\right), \\
& e_{n}^{(N)}(A)=-\sum_{\left(\boldsymbol{u}_{N}, \boldsymbol{v}_{N}\right)} J_{\boldsymbol{u}_{N}, \boldsymbol{v}_{N}} M_{\mathbf{0}, \boldsymbol{u}_{N} ;\{\mathbf{0}\}}^{(N+1)}\left(I\left[\boldsymbol{v}_{N-1} \stackrel{A}{\rightarrow} n \text { in } \tilde{C}_{N}\right] \chi_{n}\left(\boldsymbol{v}_{N} ; \tilde{C}_{N}\right)\right),
\end{aligned}
$$

where, in the last equality, we have used (4.9) (with $\left(\boldsymbol{u}_{N}, \boldsymbol{v}_{N}\right)$ instead of $\left.\left(\boldsymbol{u}_{0}, \boldsymbol{v}_{0}\right)\right)$, (3.20), and (3.35). In addition, we have repeated our abuse of notation, since the variable $\boldsymbol{v}_{N-1}$ is summed over in the definition of $M_{\mathbf{0}, \boldsymbol{u}_{N} ;\{\mathbf{0}\}}^{(N+1)}$ (see (3.21)). For $N=0$, recalling (4.8), (4.9), (4.12), and (3.20), we see that the equalities in (4.18) and (4.19) also hold, using the convention (4.15).

\subsection{The first expansion for $\gamma_{n}$}

In this section, we derive the first expansion for $\gamma_{n}$. This requires a new concept: the important set $\mathcal{P}_{A}$. We start by giving an informal explanation of this set by highlighting the analogy and differences of the first expansion to the expansion extracting the linear term performed in Section 3.

Crucial in the expansion that extracts the linear term is the identification of the cutting bond. In this section, we will describe the first cutting bond for $\gamma_{n}^{(0)}(\boldsymbol{v} ; A)$. In the expansion that extracts the linear term, we have made essential use of properties of this cutting bond. In particular, it allows for a use of the Factorisation Lemma 2.2, and, when it exists, it is unique. By the latter property, the union over the cutting bonds can be replaced by a sum, which is then analysed in detail (see e.g., (3.8)). Finally, the cutting bond is after the connection through the set $A$ has taken place, so that the dependence on $A$ has been taken care of and does not influence later connections.

In the event $F_{n}^{\prime}(\boldsymbol{v} ; A)$ that defines $\gamma_{n}^{(0)}(\boldsymbol{v} ; A)$ (recall (3.7) and (3.26)), we have that after the connection through the set $A$ has taken place, there are at least two connections to $n$ (see (3.5)). Each of these connections should give rise to a possible cutting bond, so that the cutting bond is not unique. The set $\mathcal{P}_{A}$ will consist precisely of all appropriate cutting bonds. The non-uniqueness makes it harder to go to a sum over cutting bonds, since this number is in fact 
random. However, we will argue now that the main contribution to $\gamma_{n}^{(0)}(\boldsymbol{v} ; A)$ comes from configurations where the number of appropriate cutting bonds is equal to two, and, thus, up to leading order, we can replace the union over cutting bonds by a sum if we account for the multiplicity by dividing by 2 (see (4.21) below). Indeed, when there are fewer than two cutting bonds, then one of the disjoint connections to $n$ will in fact have to be a double connection to a point $(x, n)$ for some $x \in \mathbb{Z}^{d}$, which should lead to an error term. When, on the other hand, there are at least three cutting bonds, then there are three disjoint connections to $n$, which should also lead to an error term. This explains the strategy of the proof. We now turn to the details.

Throughout the remainder of the paper, given a bond $b=((x, n),(y, n+1))$, we will write $\bar{b}=(y, n+1)$ for its "top" and $\underline{b}=(x, n)$ for its "bottom." Given a vertex $\boldsymbol{v}$, a non-negative integer $n$, and a subset $A \subseteq \Lambda$, we define the random set of bonds $\mathcal{P}_{A}$ by

$$
\mathcal{P}_{A}=\left\{\text { bonds } b \mid \text { the event } E^{\prime}(\boldsymbol{v}, \underline{b} ; A) \cap\{b \text { occupied }\} \cap\left\{\bar{b} \rightarrow n \text { in } \Lambda \backslash \tilde{C}^{b}(\boldsymbol{v})\right\} \text { occurs }\right\} .
$$

Thus $\mathcal{P}_{A}$ consists of those occupied bonds $b$ such that $E^{\prime}(\boldsymbol{v}, \underline{b} ; A)$ occurs (see Fig. 2(a)) and the top of $b$ is connected to $n$ in the complement of $\tilde{C}^{b}(v)$. If we compare this definition of the cutting bond to the properties of the cutting bond in the expansion extracting the linear term in Lemma 3.3 and (3.12), then we see that both definitions have the occurrence of $E^{\prime}(\boldsymbol{v}, \underline{b} ; A)$ and the connection $\bar{b} \rightarrow n$ in $\Lambda \backslash \tilde{C}^{b}(\boldsymbol{v})$ in common. Thus, we can think of the bonds in $\mathcal{P}_{A}$ as generalisations of the cutting bond in the linear expansion. Section 4 is primarily devoted to the investigation of the set of bonds $\mathcal{P}_{A}$.

By decomposing the event $F_{n}^{\prime}(\boldsymbol{v} ; A)$ according to the size of $\mathcal{P}_{A}$, and using (3.7), we obtain

$$
\begin{aligned}
\gamma_{n}^{(0)}(\boldsymbol{v} ; A) & =\mathbb{P}\left(F_{n}^{\prime}(\boldsymbol{v} ; A)\right) \\
& =\mathbb{P}\left(F_{n}^{\prime}(\boldsymbol{v} ; A) \cap\left\{\mathcal{P}_{A}=\varnothing\right\}\right)+\sum_{l=1}^{\infty} \frac{1}{l} \sum_{b} \mathbb{P}\left(F_{n}^{\prime}(\boldsymbol{v} ; A) \cap\left\{b \in \mathcal{P}_{A}\right\} \cap\left\{\left|\mathcal{P}_{A}\right|=l\right\}\right) \\
& =\frac{1}{2} \sum_{b} \mathbb{P}\left(F_{n}^{\prime}(\boldsymbol{v} ; A) \cap\left\{b \in \mathcal{P}_{A}\right\}\right)+e_{n}^{(0)}(\boldsymbol{v} ; A),
\end{aligned}
$$

where

$$
e_{n}^{(0)}(\boldsymbol{v} ; A)=\mathbb{P}\left(F_{n}^{\prime}(\boldsymbol{v} ; A) \cap\left\{\mathcal{P}_{A}=\varnothing\right\}\right)+\sum_{l=1}^{\infty}\left(\frac{1}{l}-\frac{1}{2}\right) \sum_{b} \mathbb{P}\left(F_{n}^{\prime}(\boldsymbol{v} ; A) \cap\left\{b \in \mathcal{P}_{A}\right\} \cap\left\{\left|\mathcal{P}_{A}\right|=l\right\}\right) .
$$

We will show that $e_{n}^{(0)}(\boldsymbol{v} ; A)$ gives rise to an error term, thus making the claim that the main contribution to $\gamma_{n}^{(0)}(\boldsymbol{v} ; A)$ comes from configurations where $\left|\mathcal{P}_{A}\right|=2$ precise.

We further define, for $N \geqslant 1$,

$$
\begin{aligned}
& e_{n}^{(0)}(1)=e_{n}^{(0)}(\mathbf{0} ;\{\mathbf{0}\}), \\
& e_{n}^{(N)}(1)=\sum_{\left(\boldsymbol{u}_{N-1}, \boldsymbol{v}_{N-1}\right)} J_{\boldsymbol{u}_{N-1}, \boldsymbol{v}_{N-1}} M_{\mathbf{0}, \boldsymbol{u}_{N-1} ;\{\boldsymbol{0}\}}^{\left(e_{n}\right.}\left(e_{n}^{(0)}\left(\boldsymbol{v}_{N-1} ; \tilde{C}_{N-1}\right)\right) .
\end{aligned}
$$

The following proposition, whose proof is deferred to Section 4.3, is a crucial ingredient in the first expansion for $\gamma_{n}$. Proposition 4.3 derives its name "the first cutting bond" from the fact that the bond $b$ in $\mathcal{P}_{A}$ will serve as the cutting bond in Proposition 4.4 .

Proposition 4.3 (The first cutting bond). For $A \subseteq \Lambda, v \in \Lambda, n \geqslant 1$ and $b$,

$$
F_{n}^{\prime}(\boldsymbol{v} ; A) \cap\left\{b \in \mathcal{P}_{A}\right\}=\left\{E^{\prime}(\boldsymbol{v}, \underline{b} ; A) \cap\{\boldsymbol{v} \stackrel{A}{\rightarrow} n\} \text { in } \tilde{C}^{b}(\boldsymbol{v})\right\} \cap\{b \text { occ. }\} \cap\left\{\bar{b} \rightarrow n \text { in } \Lambda \backslash \tilde{C}^{b}(\boldsymbol{v})\right\} .
$$

Proposition 4.3 shows that the definition of the cutting bonds in (4.20) allows us to rewrite the event $F_{n}^{\prime}(\boldsymbol{v} ; A) \cap$ $\left\{b \in \mathcal{P}_{A}\right\}$ in terms of Definition 2.1, which, in turn, allows us to use the Factorisation Lemma 2.2. Because of this, Proposition 4.3 is the key to our expansion of the survival probability.

The following proposition, whose proof uses Proposition 4.3, gives the result of the first expansion for $\gamma_{n}$. On the right-hand side of (4.26), there is again an abuse of notation: when we put $A=\tilde{C}_{N-1}$ in (4.13), (4.14), the quantities $\kappa_{m_{1}, n}^{(N)}\left(\tilde{C}_{N-1}\right)$ and $e_{n}^{(N)}\left(\tilde{C}_{N-1}\right)$ do not actually depend on $\tilde{C}_{N-1}$ (this random set is integrated over). 
Proposition 4.4 (The first expansion for $\gamma_{n}$ ). For $n \geqslant 1$ and $N \geqslant 0$,

$$
\gamma_{n}^{(N)}=\frac{1}{2} \sum_{m_{1}=1}^{n} \kappa_{m_{1}, n}^{(N)}\left(\tilde{C}_{N-1}\right) \theta_{n-m_{1}}+\frac{1}{2} e_{n}^{(N)}\left(\tilde{C}_{N-1}\right)+e_{n}^{(N)}(1) .
$$

Proof. By (4.21), Proposition 4.3, and the independence stated in Lemma 2.2,

$$
\gamma_{n}^{(0)}(\boldsymbol{v} ; A)=\frac{1}{2} \sum_{\left(\boldsymbol{u}_{0}, \boldsymbol{v}_{0}\right)} J_{\boldsymbol{u}_{0}, \boldsymbol{v}_{0}} \mathbb{E}\left(I\left[E^{\prime}\left(\boldsymbol{v}, \boldsymbol{u}_{0} ; A\right) \cap\{\boldsymbol{v} \stackrel{A}{\rightarrow} n\} \text { in } \tilde{C}_{0}\right] I\left[\boldsymbol{v}_{0} \rightarrow n \text { in } \Lambda \backslash \tilde{C}_{0}\right]\right)+e_{n}^{(0)}(\boldsymbol{v} ; A) .
$$

By Lemma 2.2, (3.17), and (4.5), this implies that

$$
\gamma_{n}^{(0)}(\boldsymbol{v} ; A)=\frac{1}{2} \kappa_{n}^{(0)}(\boldsymbol{v} ; A, A)+e_{n}^{(0)}(\boldsymbol{v} ; A) .
$$

By Lemma 4.1, (4.15) and (3.32), this proves (4.26) for $N=0$. For $N \geqslant 1$, we substitute (4.28) for $\gamma_{n}^{(0)}\left(\boldsymbol{v}_{N-1} ; \tilde{C}_{N-1}\right)$ in (3.26). The desired result then follows from (4.13), (4.14) and (4.24).

To combine the expansions for $\rho_{n}$ and $\gamma_{n}$ given in Propositions 4.2 and 4.4 into a first expansion for $\chi_{n}^{(N)}$, we introduce the following notation. Let

$$
\begin{aligned}
& \kappa_{m, n}^{(N)}=\kappa_{m, n}^{(N)}\left(\left\{\boldsymbol{v}_{N-1}\right\}\right)-\frac{1}{2} \kappa_{m, n}^{(N)}\left(\tilde{C}_{N-1}\right), \\
& e_{n}^{(N)}(2)=\frac{1}{2} e_{n}^{(N)}\left(\tilde{C}_{N-1}\right)-e_{n}^{(N)}\left(\left\{\boldsymbol{v}_{N-1}\right\}\right), \\
& e_{n}^{(N)}(3)=-\sum_{m_{1}=\lfloor n / 2\rfloor+1}^{n} \kappa_{m_{1}, n}^{(N)} \theta_{n-m_{1}} .
\end{aligned}
$$

Corollary 4.5 (The first expansion for $\chi_{n}$ ). For $n \geqslant 0$ and $N \geqslant 0$,

$$
\chi_{n}^{(N)}=-\sum_{m_{1}=1}^{\lfloor n / 2\rfloor} \kappa_{m_{1}, n}^{(N)} \theta_{n-m_{1}}+e_{n}^{(N)}(1)+e_{n}^{(N)}(2)+e_{n}^{(N)}(3) .
$$

Proof. Since $\chi_{n}^{(N)}=\gamma_{n}^{(N)}-\rho_{n}^{(N)}$ by (3.29), we can combine the conclusions of Propositions 4.2 and 4.4 with (4.29), (4.30) to arrive at

$$
\chi_{n}^{(N)}=-\sum_{m_{1}=1}^{n} \kappa_{m_{1}, n}^{(N)} \theta_{n-m_{1}}+e_{n}^{(N)}(1)+e_{n}^{(N)}(2) .
$$

Then (4.32) follows from (4.31).

\subsection{Proof of Proposition 4.3}

The proof is divided into 2 steps. See Fig. 6 for a schematic representation of the event $F_{n}^{\prime}(\boldsymbol{v} ; A) \cap\left\{b \in \mathcal{P}_{A}\right\}$.

Step 1: The left-hand side of (4.25) is a subset of the right-hand side of (4.25). Suppose that the left-hand side of (4.25) occurs. It is clear from (4.20) that all the events on the right-hand side of (4.25) occur, apart from the event $\left\{\boldsymbol{v} \stackrel{A}{\rightarrow} n\right.$ in $\left.\tilde{C}^{b}(\boldsymbol{v})\right\}$. To see that $\left\{\boldsymbol{v} \stackrel{A}{\rightarrow} n\right.$ in $\left.\tilde{C}^{b}(\boldsymbol{v})\right\}$ occurs, note from (3.5) that $F_{n}^{\prime}(\boldsymbol{v} ; A)$ implies that $\{\boldsymbol{v} \stackrel{A}{\rightarrow} n\}$. Also, by (4.20), $\underline{b} \rightarrow \bar{b} \rightarrow n$ on the left-hand side of (4.25). Since $F_{n}^{\prime}(\boldsymbol{v} ; A)$ occurs and $\boldsymbol{v} \stackrel{A}{\rightarrow} \underline{b}$, it follows from (3.5) that $b$ cannot be pivotal for $\boldsymbol{v} \rightarrow n$. Thus $\left\{\boldsymbol{v} \rightarrow n\right.$ in $\left.\tilde{C}^{b}(\boldsymbol{v})\right\}$ must occur. Since $\boldsymbol{v} \stackrel{A}{\rightarrow} n$, every occupied path $\boldsymbol{v} \rightarrow n$ must contain an element in $A$, in particular the paths in $\tilde{C}^{b}(\boldsymbol{v})$. We conclude that $\left\{\boldsymbol{v} \stackrel{A}{\rightarrow} n\right.$ in $\left.\tilde{C}^{b}(\boldsymbol{v})\right\}$ occurs. This proves that the left-hand side of (4.25) is a subset of the right-hand side.

Step 2: The right-hand side of (4.25) is a subset of the left-hand side of (4.25). Suppose that the right-hand side of (4.25) occurs. Then $b \in \mathcal{P}_{A}$ by the definition of $\mathcal{P}_{A}$ in (4.20). It remains to check that $F_{n}^{\prime}(\boldsymbol{v} ; A)$ occurs. To achieve 


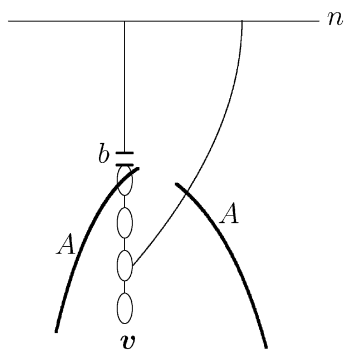

Fig. 6. Schematic representation of the event $F_{n}^{\prime}(\boldsymbol{v} ; A) \cap\left\{b \in \mathcal{P}_{A}\right\}$.

this, we need to verify that (a) $v \stackrel{A}{\rightarrow} n$, and (b) the "no previous pivotal" condition in (3.5) holds (i.e., there does not exists a $b^{\prime}$ which is occupied and pivotal for $\boldsymbol{v} \rightarrow n$ such that $\left.\boldsymbol{v} \stackrel{A}{\rightarrow} \underline{b}^{\prime}\right)$.

For (a), we note that since $\boldsymbol{v} \stackrel{A}{\rightarrow} n$ in $\tilde{C}^{b}(\boldsymbol{v})$, all connections that do not use the bond $b$ are through $A$. Thus, we need only investigate the connections that $d o$ use the bond $b$. But (3.11) implies that $\boldsymbol{v} \stackrel{A}{\rightarrow} \underline{b}$, so the connections using the bond $b$ are indeed through $A$, and hence $v \stackrel{A}{\rightarrow} n$.

We are left to check (b). We first note that if $b^{\prime}$ is pivotal for $\boldsymbol{v} \rightarrow n$ on the right-hand side of (4.25), then $b^{\prime}$ is also pivotal for $\boldsymbol{v} \rightarrow \underline{b}$. Indeed, suppose that after removal of $b^{\prime}$, the connection $\boldsymbol{v} \rightarrow \underline{b}$ still occurs. The bond $b^{\prime}$ cannot equal $b$ since $\boldsymbol{v} \rightarrow n$ in $\tilde{C}^{b}(\boldsymbol{v})$, so that $b$ is not pivotal for $\boldsymbol{v} \rightarrow n$, whereas $b^{\prime}$ is. Thus, since $b^{\prime}$ is pivotal for $\boldsymbol{v} \rightarrow n$, the removal of $b^{\prime}$ must destroy both connections $\boldsymbol{v} \rightarrow n$ in $\tilde{C}^{b}(\boldsymbol{v})$ and $\bar{b} \rightarrow n$ in $\Lambda \backslash \tilde{C}^{b}(\boldsymbol{v})$, which is impossible.

To prove (b), we need to show that if $b^{\prime}$ is pivotal for $\boldsymbol{v} \rightarrow n$, then $\boldsymbol{v}$ is not connected to $\underline{b}^{\prime}$ through $A$. Let $b^{\prime}$ be pivotal for $\boldsymbol{v} \rightarrow n$. Then, as noted above, $b^{\prime}$ is also pivotal for $\boldsymbol{v} \rightarrow \underline{b}$. By (3.11) and the second event in (3.4), $\left\{\boldsymbol{v} \stackrel{A}{\rightarrow} \underline{b}^{\prime}\right\}^{c}$ must occur. This proves (b) and completes the proof of Proposition 4.3.

\subsection{Preparation for bounds on $e_{n}^{(0)}(\boldsymbol{v} ; A)$}

In this section, we set the stage for the diagrammatic estimates of Section 8, by proving estimates for the error term $e_{n}^{(0)}(\boldsymbol{v} ; A)$ of (4.22). We begin by making the decomposition

$$
e_{n}^{(0)}(\boldsymbol{v} ; A)=e_{n}^{(0)}(\boldsymbol{v} ; A ; 1)+e_{n}^{(0)}(\boldsymbol{v} ; A ; 2)+e_{n}^{(0)}(\boldsymbol{v} ; A ; 3),
$$

where

$$
\begin{aligned}
& e_{n}^{(0)}(\boldsymbol{v} ; A ; 1)=\mathbb{P}\left(F_{n}^{\prime}(\boldsymbol{v} ; A) \cap\left\{\mathcal{P}_{A}=\varnothing\right\}\right)+\frac{1}{2} \mathbb{P}\left(F_{n}^{\prime}(\boldsymbol{v} ; A) \cap\left\{\left|\mathcal{P}_{A}\right|=1\right\}\right), \\
& e_{n}^{(0)}(\boldsymbol{v} ; A ; 2)=\sum_{b \geqslant\lfloor n / 2\rfloor+1} \sum_{l=3}^{\infty}\left(\frac{1}{l}-\frac{1}{2}\right) \mathbb{P}\left(F_{n}^{\prime}(\boldsymbol{v} ; A) \cap\left\{b \in \mathcal{P}_{A}\right\} \cap\left\{\left|\mathcal{P}_{A}\right|=l\right\}\right), \\
& e_{n}^{(0)}(\boldsymbol{v} ; A ; 3)=\sum_{b \leqslant\lfloor n / 2\rfloor} \sum_{l=3}^{\infty}\left(\frac{1}{l}-\frac{1}{2}\right) \mathbb{P}\left(F_{n}^{\prime}(\boldsymbol{v} ; A) \cap\left\{b \in \mathcal{P}_{A}\right\} \cap\left\{\left|\mathcal{P}_{A}\right|=l\right\}\right),
\end{aligned}
$$

and where we abuse notation by writing $b \leqslant m$ for the sum over bonds $b$ such that $m_{\underline{b}} \leqslant m$ (recall that $m_{\underline{b}}$ denotes the temporal component of $\underline{b}$ ). Then

$$
\begin{aligned}
& \left|e_{n}^{(0)}(\boldsymbol{v} ; A ; 1)\right| \leqslant \mathbb{P}\left(F_{n}^{\prime}(\boldsymbol{v} ; A) \cap\left\{\left|\mathcal{P}_{A}\right| \leqslant 1\right\}\right), \\
& \left|e_{n}^{(0)}(\boldsymbol{v} ; A ; 2)\right| \leqslant \frac{1}{2} \sum_{b \geqslant\lfloor n / 2\rfloor+1} \mathbb{P}\left(E^{\prime}(\boldsymbol{v}, \underline{b} ; A) \cap\{\underline{b} \rightarrow n\}\right), \\
& \left|e_{n}^{(0)}(\boldsymbol{v} ; A ; 3)\right| \leqslant \frac{1}{2} \sum_{b \leqslant\lfloor n / 2\rfloor} \mathbb{P}\left(F_{n}^{\prime}(\boldsymbol{v} ; A) \cap\left\{b \in \mathcal{P}_{A}\right\} \cap\left\{\left|\mathcal{P}_{A}\right| \geqslant 3\right\}\right),
\end{aligned}
$$

using Proposition 4.3 in (4.39). We consider these three quantities in sequence in Sections 4.4.1-4.4.3. 
4.4.1. Estimate for $e_{n}^{(0)}(v ; A ; 1)$

To prove that $e_{n}^{(0)}(\boldsymbol{v} ; A ; 1)$ produces an error term, we will use the following proposition.

Proposition 4.6. For $v \in \Lambda, A \subseteq \Lambda$ and $n \geqslant 1$,

$$
F_{n}^{\prime}(\boldsymbol{v} ; A) \cap\left\{\left|\mathcal{P}_{A}\right| \leqslant 1\right\} \subseteq \bigcup_{x \in \mathbb{Z}^{d}} E^{\prime}(\boldsymbol{v},(x, n) ; A) .
$$

Proof. By partitioning (3.5) according to the last pivotal bond for the connection $\boldsymbol{v} \rightarrow n$, we may write

$$
F_{n}^{\prime}(\boldsymbol{v} ; A)=\{\boldsymbol{v} \stackrel{A}{\Longrightarrow} n\} \dot{\cup} \bigcup_{b}\left(\{b \text { occ. and piv. for } \boldsymbol{v} \rightarrow n\} \cap\{\boldsymbol{v} \stackrel{A}{\rightarrow} \underline{b}\}^{c} \cap\{\bar{b} \stackrel{A}{\Longrightarrow} n\}\right),
$$

where $\{\boldsymbol{v} \stackrel{A}{\Rightarrow} n\}=\{\boldsymbol{v} \stackrel{A}{\rightarrow} n\} \cap\{\boldsymbol{v} \Rightarrow n\}$. We define $\boldsymbol{v}^{\prime}$ by setting $\boldsymbol{v}^{\prime}=\boldsymbol{v}$ when the first event on the right-hand side of (4.42) occurs, and $\boldsymbol{v}^{\prime}=\bar{b}$ otherwise.

Suppose that $F_{n}^{\prime}(\boldsymbol{v} ; A) \cap\left\{\left|\mathcal{P}_{A}\right| \leqslant 1\right\}$ occurs. There must be $x, y$ such that $\boldsymbol{v}^{\prime}$ is disjointly connected through $A$ to $(x, n)$ and $(y, n)$. Due to these disjoint connections, no pivotal bond for $\boldsymbol{v}^{\prime} \rightarrow(x, n)$ can also be pivotal for $\boldsymbol{v}^{\prime} \rightarrow(y, n)$. Since $\left|\mathcal{P}_{A}\right| \leqslant 1$, we may therefore assume without loss of generality that among the pivotal bonds for $\boldsymbol{v}^{\prime} \rightarrow(x, n)$ (if there are any) there is no element of $\mathcal{P}_{A}$.

According to (3.4), it suffices to show that there is no pivotal bond $b^{\prime}$ for $\boldsymbol{v} \rightarrow(x, n)$ such that $\boldsymbol{v} \stackrel{A}{\rightarrow} \underline{b}^{\prime}$, since this implies that $E^{\prime}(\boldsymbol{v},(x, n) ; A)$ occurs. We will establish that this sufficient condition holds, by arguing by contradiction.

Suppose that $b^{\prime}$ is pivotal for $\boldsymbol{v} \rightarrow(x, n)$ and that $\boldsymbol{v} \stackrel{A}{\rightarrow} \underline{b}^{\prime}$. Then there must be a first such pivotal bond, which, we claim, is an element of $\mathcal{P}_{A}$. Indeed, since $b^{\prime}$ is pivotal for $\boldsymbol{v} \rightarrow(x, n)$, it follows from (3.1) that $b^{\prime}$ is occupied, $E^{\prime}\left(\boldsymbol{v}, \underline{b}^{\prime} ; A\right)$ occurs, and $\bar{b}^{\prime} \rightarrow(x, n)$ occurs in $\Lambda \backslash \tilde{C}^{b}(\boldsymbol{v})$. This shows that $b^{\prime} \in \mathcal{P}_{A}$.

By definition of $\boldsymbol{v}^{\prime}$, the pivotal bonds for $\boldsymbol{v} \rightarrow(x, n)$ include the pivotal bonds for $\boldsymbol{v}^{\prime} \rightarrow(x, n)$. The latter include no element of $\mathcal{P}_{A}$, so $b^{\prime}$ must lie below $\boldsymbol{v}^{\prime}$ (and hence $\boldsymbol{v}^{\prime} \neq \boldsymbol{v}$ ). This then implies that $b^{\prime}$ is occupied and pivotal for $\boldsymbol{v} \rightarrow \boldsymbol{v}^{\prime}$. However, by (4.42), the latter implies that $\left\{\boldsymbol{v} \stackrel{A}{\rightarrow} \underline{b}^{\prime}\right\}^{c}$ occurs. This contradicts the assumption that $\boldsymbol{v} \stackrel{A}{\rightarrow} \underline{b}^{\prime}$, and completes the proof.

4.4.2. Estimate for $e_{n}^{(0)}(\boldsymbol{v} ; A ; 2)$

The right-hand side of (4.39) is already simple and nothing more is required at this stage.

4.4.3. Estimate for $e_{n}^{(0)}(\boldsymbol{v} ; A ; 3)$

We prove three lemmas, Lemmas 4.7-4.9 below, before proving the main estimate in Proposition 4.10 below.

Lemma 4.7. If $b \in \mathcal{P}_{A}$, then there exists an $x \in \mathbb{Z}^{d}$ such that $b$ is occupied and pivotal for $\boldsymbol{v} \rightarrow(x, n)$.

Proof. The definition of $\mathcal{P}_{A}$ in (4.20) implies that $\boldsymbol{v} \rightarrow \underline{b}$ occurs in $\tilde{C}^{b}(\boldsymbol{v})$, while $\bar{b} \rightarrow n$ occurs in $\Lambda \backslash \tilde{C}^{b}(\boldsymbol{v})$. Therefore, there exists an $x \in \mathbb{Z}^{d}$ for which $\bar{b} \rightarrow(x, n)$ occurs in $\Lambda \backslash \tilde{C}^{b}(\boldsymbol{v})$. By (3.1), this proves the claim.

For two bonds $b$ and $b^{\prime}$, we write $b \leqslant b^{\prime}$ when their temporal components obey $m_{\underline{b}} \leqslant m_{\underline{b^{\prime}}}$.

Lemma 4.8. For $b^{\prime} \leqslant b$ and $b^{\prime} \neq b$,

$$
\left\{b, b^{\prime} \in \mathcal{P}_{A}\right\} \subseteq\left\{\left\{b^{\prime} \in \mathcal{P}_{A}\right\} \text { in } \tilde{C}^{b}(\boldsymbol{v})\right\} .
$$

Proof. We first note that if $b$ and $b^{\prime}$ are distinct elements of $\mathcal{P}_{A}$, then it is not possible that $\bar{b}=\bar{b}^{\prime}$. Indeed, by Lemma 4.7 there is an $x$ such that $b$ is pivotal for $v \rightarrow(x, n)$. But if $\bar{b}^{\prime}=\bar{b}$ then it follows from the fact that $b^{\prime} \in \mathcal{P}_{A}$ that there is a connection from $v$ to $\bar{b}$ via $b^{\prime}$ that persists after $b$ is made vacant, and this means that $b$ cannot be pivotal for $\boldsymbol{v} \rightarrow(x, n)$. Thus we may assume that $\bar{b} \neq \bar{b}^{\prime}$.

Since $b^{\prime} \in \mathcal{P}_{A}$, we have that (a) $\left\{b^{\prime}\right.$ is occupied $\}$ occurs, (b) $E^{\prime}\left(\boldsymbol{v} ; \underline{b}^{\prime} ; A\right)$ occurs, and (c) $\left\{\bar{b}^{\prime} \rightarrow n\right.$ in $\left.\Lambda \backslash \tilde{C}^{b^{\prime}}(\boldsymbol{v})\right\}$ occurs. The event $\left\{b^{\prime}\right.$ is occupied $\}$ also occurs in $\tilde{C}^{b}(\boldsymbol{v})$ since $b \neq b^{\prime}$, and the event $E^{\prime}\left(\boldsymbol{v} ; \underline{b}^{\prime} ; A\right)$ also occurs in $\tilde{C}^{b}(\boldsymbol{v})$ since $b^{\prime} \leqslant b$ and $\bar{b} \neq \bar{b}^{\prime}$. It remains to show that the event

$$
\left\{\left\{\bar{b}^{\prime} \rightarrow n \text { in } \Lambda \backslash \tilde{C}^{b^{\prime}}(\boldsymbol{v})\right\} \text { in } \tilde{C}^{b}(\boldsymbol{v})\right\}
$$


occurs. We show that (4.44) occurs by intersecting with the events (i) $\left\{\bar{b}^{\prime} \rightarrow \underline{b} \text { in } \Lambda \backslash \tilde{C}^{b^{\prime}}(v)\right\}^{c}$, and (ii) $\left\{\bar{b}^{\prime} \rightarrow \underline{b}\right.$ in $\left.\Lambda \backslash \tilde{C}^{b^{\prime}}(\boldsymbol{v})\right\}$, which we refer to as cases (i) and (ii).

On the event (i),

$$
\left\{\left\{\bar{b}^{\prime} \rightarrow n \text { in } \Lambda \backslash \tilde{C}^{b^{\prime}}(\boldsymbol{v})\right\} \text { in } \tilde{C}^{b}(\boldsymbol{v})\right\}=\left\{\bar{b}^{\prime} \rightarrow n \text { in } \Lambda \backslash \tilde{C}^{b^{\prime}}(\boldsymbol{v})\right\},
$$

since making $b$ vacant does not change $C\left(\bar{b}^{\prime}\right) \cap\left(\Lambda \backslash \tilde{C}^{b^{\prime}}(\boldsymbol{v})\right)$, and $\bar{b}^{\prime} \rightarrow n$ in $\Lambda \backslash \tilde{C}^{b^{\prime}}(\boldsymbol{v})$ is determined by $C\left(\bar{b}^{\prime}\right) \cap$ $\left(\Lambda \backslash \tilde{C}^{b^{\prime}}(\boldsymbol{v})\right)$. But the right-hand side of (4.45) occurs by (c) above, and hence (4.44) occurs.

We complete the proof by showing that case (ii) is empty, arguing by contradiction. Suppose that $b, b^{\prime} \in \mathcal{P}_{A}$ and that $\bar{b}^{\prime} \rightarrow \underline{b}$ occurs in $\Lambda \backslash \tilde{C}^{b^{\prime}}(\boldsymbol{v})$. Then $E^{\prime}(\boldsymbol{v} ; \underline{b} ; A)$ occurs since $b \in \mathcal{P}_{A}$, and $\boldsymbol{v} \stackrel{A}{\rightarrow} \underline{b}^{\prime}$ occurs since $b^{\prime} \in \mathcal{P}_{A}$. Since $E^{\prime}(\boldsymbol{v} ; \underline{b} ; A) \cap\left\{\boldsymbol{v} \stackrel{A}{\rightarrow} \underline{b}^{\prime}\right\}$ occurs, $b^{\prime}$ cannot be pivotal for $\boldsymbol{v} \rightarrow \underline{b}$. Since $\boldsymbol{v} \rightarrow \underline{b}$, we conclude that $\underline{b} \in \tilde{C}^{b^{\prime}}(\boldsymbol{v})$. However, when $\bar{b}^{\prime} \rightarrow \underline{b}$ in $\Lambda \backslash \tilde{C}^{b^{\prime}}(\boldsymbol{v})$, either $\underline{b} \in \Lambda \backslash \tilde{C}^{b^{\prime}}(\boldsymbol{v})$ or $\bar{b}^{\prime}=\underline{b}$. In the latter case, since $b^{\prime} \in \mathcal{P}_{A}$, it follows from Proposition 4.3 that $\underline{b}=\bar{b}^{\prime} \notin \tilde{C}^{b^{\prime}}(\boldsymbol{v})$. Therefore $\underline{b} \in \Lambda \backslash \tilde{C}^{b^{\prime}}(\boldsymbol{v})$ in either case, which contradicts $\underline{b} \in \tilde{C}^{b^{\prime}}(\boldsymbol{v})$ and completes the proof.

Lemma 4.9. For $v \in \Lambda, A \subset \Lambda, n \geqslant 0$,

$$
\{\boldsymbol{v} \stackrel{A}{\rightarrow} n\} \cap\left\{\left|\mathcal{P}_{A}\right| \geqslant 2\right\} \subseteq F_{n}^{\prime}(\boldsymbol{v} ; A) .
$$

Proof. By Lemma 3.3,

$$
\begin{aligned}
\{\boldsymbol{v} & \stackrel{A}{\rightarrow} n\} \cap\left\{\left|\mathcal{P}_{A}\right| \geqslant 2\right\} \\
& =\left(F_{n}^{\prime}(\boldsymbol{v} ; A) \cap\left\{\left|\mathcal{P}_{A}\right| \geqslant 2\right\}\right) \dot{\cup} \bigcup_{b}^{\cdot}\left[E^{\prime}(\boldsymbol{v}, \underline{b} ; A) \cap\{b \text { occ. and piv. for } \boldsymbol{v} \rightarrow n\} \cap\left\{\left|\mathcal{P}_{A}\right| \geqslant 2\right\}\right] .
\end{aligned}
$$

It suffices to show that the contribution from the union over $b$ is empty. For this, it suffices to show that if $E^{\prime}(\boldsymbol{v}, \underline{b} ; A) \cap$ $\{b$ occ. and piv. for $\boldsymbol{v} \rightarrow n\}$ occurs, then $\mathcal{P}_{A}=\{b\}$.

To prove the latter statement, assume that $E^{\prime}(\boldsymbol{v}, \underline{b} ; A) \cap\{b$ occ. and piv. for $\boldsymbol{v} \rightarrow n\}$ occurs. Then clearly $b \in \mathcal{P}_{A}$, since all the events in (4.20) occur by (3.2). Also, if $b^{\prime} \in \mathcal{P}_{A}$, then the event $E^{\prime}\left(\boldsymbol{v}, \underline{b}^{\prime} ; A\right)$ occurs, and, by Lemma 4.7, $b^{\prime}$ is occupied and pivotal for $\boldsymbol{v} \rightarrow(x, n)$ for some $x \in \mathbb{Z}^{d}$. Therefore, $b^{\prime}$ is the first occupied and pivotal bond for $\boldsymbol{v} \rightarrow(x, n)$ for which $\boldsymbol{v} \stackrel{A}{\rightarrow} \underline{b}^{\prime}$. However, since $E^{\prime}(\boldsymbol{v}, \underline{b} ; A) \cap\{b$ occ. and piv. for $\boldsymbol{v} \rightarrow n\}$ occurs, $b$ is the first occupied and pivotal bond for $\boldsymbol{v} \rightarrow(x, n)$ such that $\boldsymbol{v} \stackrel{A}{\rightarrow} \underline{b}$ for all $x \in \mathbb{Z}^{d}$ for which $\boldsymbol{v} \rightarrow(x, n)$. Therefore, $b^{\prime}=b$.

To formulate the next proposition, we define

$$
\mathcal{P}_{A}^{(3)}=\left\{b \in \mathcal{P}_{A}: \exists b_{1}, b_{2} \in \mathcal{P}_{A} \backslash\{b\} \text { such that } b_{1} \neq b_{2}, b_{1}, b_{2} \leqslant b\right\} .
$$

In words, $\mathcal{P}_{A}^{(3)}$ is the subset of bonds $b \in \mathcal{P}_{A}$ for which there are at least two distinct elements in $\mathcal{P}_{A}$ with time variables smaller than or equal to $b$. Note that if $\left|\mathcal{P}_{A}\right| \geqslant 3$ then $\mathcal{P}_{A}^{(3)} \neq \varnothing$. Therefore, writing $b^{\prime} \lesseqgtr b$ to mean both $b^{\prime} \leqslant b$ and $b^{\prime} \neq b$, we have

$$
\left\{b \in \mathcal{P}_{A}\right\} \cap\left\{\left|\mathcal{P}_{A}\right| \geqslant 3\right\}=\left(\left\{b \in \mathcal{P}_{A}^{(3)}\right\} \cap \bigcup_{b^{\prime} \Varangle b}\left\{b^{\prime} \in \mathcal{P}_{A}\right\}\right) \cup\left(\bigcup_{b^{\prime} \ngtr b}\left\{b^{\prime} \in \mathcal{P}_{A}^{(3)}\right\} \cap\left\{b \in \mathcal{P}_{A}\right\}\right) .
$$

We recall (4.40) and conclude from (4.49) that

$$
\left|e_{n}^{(0)}(\boldsymbol{v} ; A ; 3)\right| \leqslant \sum_{b^{\prime} \leqslant\lfloor n / 2\rfloor} \sum_{b \geqslant b^{\prime}} \mathbb{P}\left(F_{n}^{\prime}(\boldsymbol{v} ; A) \cap\left\{b^{\prime} \in \mathcal{P}_{A}\right\} \cap\left\{b \in \mathcal{P}_{A}^{(3)}\right\}\right),
$$

where we replace $b \leqslant\left\lfloor\frac{n}{2}\right\rfloor$ by $b^{\prime} \leqslant\left\lfloor\frac{n}{2}\right\rfloor$ for the contribution due to the first event in the right-hand side of (4.49), and the roles of $b$ and $b^{\prime}$ are interchanged for the contribution due to the second event.

The following proposition gives an estimate on the event appearing in the right-hand side of (4.50). In the righthand side of (4.51) below, three connections to $n$ are apparent. One is due to $\left\{\bar{b} \rightarrow n\right.$ in $\left.\Lambda \backslash \tilde{C}^{b}(\boldsymbol{v})\right\}$, and the other two are due to the event $\left\{F_{n}^{\prime}(\boldsymbol{v} ; A)\right.$ in $\left.\tilde{C}^{b}(\boldsymbol{v})\right\}$. The advantage of the right-hand side of (4.51) is that it is well suited for application of the Factorisation Lemma 2.2. In Section 8.7, we will exploit this formula to prove that $e_{n}^{(0)}(\boldsymbol{v} ; A ; 3)$ gives an error term. 
Proposition 4.10 (Factorisation for three cutting bonds). For $A \subseteq \Lambda, v \in \Lambda, n \geqslant 1$ and $b^{\prime} \lesseqgtr b$,

$$
\begin{aligned}
& F_{n}^{\prime}(\boldsymbol{v} ; A) \cap\left\{b^{\prime} \in \mathcal{P}_{A}\right\} \cap\left\{b \in \mathcal{P}_{A}^{(3)}\right\} \\
& \quad \subseteq\left\{E^{\prime}(\boldsymbol{v}, \underline{b} ; A) \cap F_{n}^{\prime}(\boldsymbol{v} ; A) \cap\left\{b^{\prime} \in \mathcal{P}_{A}\right\} \text { in } \tilde{C}^{b}(\boldsymbol{v})\right\} \cap\{b \text { occ. }\} \cap\left\{\bar{b} \rightarrow n \text { in } \Lambda \backslash \tilde{C}^{b}(\boldsymbol{v})\right\} .
\end{aligned}
$$

Proof. By Lemma 4.8,

$$
\begin{aligned}
\left\{b^{\prime} \in \mathcal{P}_{A}\right\} \cap\left\{b \in \mathcal{P}_{A}^{(3)}\right\} & =\left\{b \in \mathcal{P}_{A}\right\} \cap\left\{b^{\prime} \in \mathcal{P}_{A}\right\} \cap\left(\bigcup_{\substack{b^{\prime \prime} \leqslant b: \\
b^{\prime \prime} \neq b, b^{\prime}}}\left\{b^{\prime \prime} \in \mathcal{P}_{A}\right\}\right) \\
& \subseteq\left\{b \in \mathcal{P}_{A}\right\} \cap\left\{\left\{b^{\prime} \in \mathcal{P}_{A}\right\} \text { in } \tilde{C}^{b}(\boldsymbol{v})\right\} \cap\left(\bigcup_{\substack{b^{\prime \prime} \leqslant b: \\
b^{\prime \prime} \neq b, b^{\prime}}}\left\{\left\{b^{\prime \prime} \in \mathcal{P}_{A}\right\} \text { in } \tilde{C}^{b}(\boldsymbol{v})\right\}\right) \\
& =\left\{b \in \mathcal{P}_{A}\right\} \cap\left\{\left\{\bigcup_{\substack{b^{\prime \prime} \leqslant b: \\
b^{\prime \prime} \neq b, b^{\prime}}}\left\{b^{\prime}, b^{\prime \prime} \in \mathcal{P}_{A}\right\}\right\} \text { in } \tilde{C}^{b}(\boldsymbol{v})\right\} \\
& \subseteq\left\{\left\{b^{\prime} \in \mathcal{P}_{A}\right\} \cap\left\{\left|\mathcal{P}_{A}\right| \geqslant 2\right\} \text { in } \tilde{C}^{b}(\boldsymbol{v})\right\},
\end{aligned}
$$

where we used (2.3), (2.4) in the third line. Now we use Proposition 4.3, (4.52), (2.3), and Lemma 4.9 to arrive at

$$
\begin{aligned}
& F_{n}^{\prime}(\boldsymbol{v} ; A) \cap\left\{b^{\prime} \in \mathcal{P}_{A}\right\} \cap\left\{b \in \mathcal{P}_{A}^{(3)}\right\} \\
&=\left(F_{n}^{\prime}(\boldsymbol{v} ; A) \cap\left\{b \in \mathcal{P}_{A}\right\}\right) \cap\left(\left\{b^{\prime} \in \mathcal{P}_{A}\right\} \cap\left\{b \in \mathcal{P}_{A}^{(3)}\right\}\right) \\
& \subseteq\left\{E^{\prime}(\boldsymbol{v}, \underline{b} ; A) \cap\{\boldsymbol{v} \stackrel{A}{\rightarrow} n\} \text { in } \tilde{C}^{b}(\boldsymbol{v})\right\} \cap\{b \text { occ. }\} \cap\left\{\bar{b} \rightarrow n \text { in } \Lambda \backslash \tilde{C}^{b}(\boldsymbol{v})\right\} \\
& \cap\left\{\left\{b^{\prime} \in \mathcal{P}_{A}\right\} \cap\left\{\left|\mathcal{P}_{A}\right| \geqslant 2\right\} \text { in } \tilde{C}^{b}(\boldsymbol{v})\right\} \\
&=\left\{E^{\prime}(\boldsymbol{v}, \underline{b} ; A) \cap\{\boldsymbol{v} \stackrel{A}{\rightarrow} n\} \cap\left\{\left|\mathcal{P}_{A}\right| \geqslant 2\right\} \cap\left\{b^{\prime} \in \mathcal{P}_{A}\right\} \text { in } \tilde{C}^{b}(\boldsymbol{v})\right\} \cap\{b \text { occ. }\} \cap\left\{\bar{b} \rightarrow n \text { in } \Lambda \backslash \tilde{C}^{b}(\boldsymbol{v})\right\} \\
& \subseteq\left\{E^{\prime}(\boldsymbol{v}, \underline{b} ; A) \cap F_{n}^{\prime}(\boldsymbol{v} ; A) \cap\left\{b^{\prime} \in \mathcal{P}_{A}\right\} \text { in } \tilde{C}^{b}(\boldsymbol{v})\right\} \cap\{b \text { occ. }\} \cap\left\{\bar{b} \rightarrow n \text { in } \Lambda \backslash \tilde{C}^{b}(\boldsymbol{v})\right\},
\end{aligned}
$$

which is the desired result.

\section{The quadratic term: The second expansion for $\chi_{n}$}

In this section, we complete the proof of Theorem 1.1(i) by proving (4.2). To do so, we will determine coefficients $\phi_{m_{1}, m_{2}}^{(N)}$ and $d_{m_{1}, n}^{(N)}$ such that

$$
\kappa_{m_{1}, n}^{(N)}=\sum_{m_{2}=m_{1}}^{n} \phi_{m_{1}, m_{2}}^{(N)} \theta_{n-m_{2}}-d_{m_{1}, n}^{(N)} .
$$

Then (4.2) follows from (4.32), with

$$
e_{n}^{(N)}=e_{n}^{(N)}(1)+e_{n}^{(N)}(2)+e_{n}^{(N)}(3)+\sum_{m_{1}=1}^{\lfloor n / 2\rfloor} d_{m_{1}, n}^{(N)} \theta_{n-m_{1}} .
$$

We will also prove the first statement of Theorem 1.1(ii), namely that $\phi_{1,1}=\frac{1}{2} p_{c}^{2} \sum_{x} D(x)(1-D(x))$. By (1.4), (1.7) and (1.9), this implies that $\phi_{1,1}=\frac{1}{2}+O(\beta)$.

\subsection{The second cutting bond for $\chi_{n}$}

To prove (5.1), we will define a second cutting bond for

$$
\kappa_{m, n}^{(0)}\left(\boldsymbol{v}_{N-1} ; A, \tilde{C}_{N-1}\right)=\sum_{\left(\boldsymbol{u}_{N}, \boldsymbol{v}_{N}\right)} J_{\boldsymbol{u}_{N}, \boldsymbol{v}_{N}} \mathbb{E}_{N}\left(I\left[E^{\prime}\left(\boldsymbol{v}_{N-1}, \boldsymbol{u}_{N} ; \tilde{C}_{N-1}\right) \cap\left\{\boldsymbol{v}_{N-1} \stackrel{A}{\rightarrow} n\right\} \text { in } \tilde{C}_{N}\right] \Pi_{m}\left(\boldsymbol{v}_{N} ; \tilde{C}_{N}\right)\right)
$$


(see (4.8)), which is the argument of $M_{\mathbf{0}, \boldsymbol{u}_{N-1} ;\{\mathbf{0}\}}^{(N)}$ appearing in (4.13). The set $\tilde{C}_{N-1}$ can be any deterministic set in (5.3), but we write it in this form with (4.13) in mind. The definition of the second cutting bond will be simpler than the definition of the first cutting bond in Proposition 4.3, due to the fact that we have already extracted a factor of $\theta_{n-m_{1}}$ and any remaining contribution with a double connection to $n$ will be an error term.

We first rewrite $\kappa_{m, n}^{(0)}\left(\boldsymbol{v}_{N-1} ; A, \tilde{C}_{N-1}\right)$ in a more convenient form. Let $\tilde{\mathbb{P}}_{N}$ be the conditional probability $\mathbb{P}_{N}$ given that $\left(\boldsymbol{u}_{N}, \boldsymbol{v}_{N}\right)$ is vacant, and let $\widetilde{\mathbb{E}}_{N}$ be expectation with respect to $\tilde{\mathbb{P}}_{N}$. Since $\tilde{C}_{N}=C_{N}\left(\boldsymbol{v}_{N-1}\right)$ holds $\tilde{\mathbb{P}}_{N}$-a.s, and since the event $E^{\prime}\left(\boldsymbol{v}_{N-1}, \boldsymbol{u}_{N} ; \tilde{C}_{N-1}\right) \cap\left\{\boldsymbol{v}_{N-1} \stackrel{A}{\rightarrow} n\right\}$ only depends on forward connections from $\boldsymbol{v}_{N-1}$ to later vertices, it follows that

$$
\kappa_{m, n}^{(0)}\left(\boldsymbol{v}_{N-1} ; A, \tilde{C}_{N-1}\right)=\sum_{\left(\boldsymbol{u}_{N}, \boldsymbol{v}_{N}\right)} J_{\boldsymbol{u}_{N}, \boldsymbol{v}_{N}} \widetilde{\mathbb{E}}_{N}\left(I\left[E^{\prime}\left(\boldsymbol{v}_{N-1}, \boldsymbol{u}_{N} ; \tilde{C}_{N-1}\right) \cap\left\{\boldsymbol{v}_{N-1} \stackrel{A}{\rightarrow} n\right\}\right] \Pi_{m}\left(\boldsymbol{v}_{N} ; \tilde{C}_{N}\right)\right) .
$$

The second cutting bond is defined as follows.

Definition 5.1. (i) For $m \geqslant 0$, the $m$-cutting bond for $\boldsymbol{v}_{N-1} \stackrel{A}{\rightarrow} n$, if it exists, is the first occupied and pivotal bond $b$ for $\boldsymbol{v}_{N-1} \rightarrow n$ for which $m_{\bar{b}} \geqslant m$ and $\boldsymbol{v}_{N-1} \stackrel{A}{\rightarrow} \underline{b}$. Similarly, for $\boldsymbol{y} \in \Lambda$, the $m$-cutting bond for $\boldsymbol{v}_{N-1} \stackrel{A}{\rightarrow} \boldsymbol{y}$, if it exists, is the first occupied and pivotal bond $b$ for $\boldsymbol{v}_{N-1} \rightarrow \boldsymbol{y}$ for which $m_{\bar{b}} \geqslant m$ and $\boldsymbol{v}_{N-1} \stackrel{A}{\rightarrow} \underline{b}$. We use the abbreviation " $b$ is $m$-cutting for $\boldsymbol{v}_{N-1} \stackrel{A}{\rightarrow} n$ " for the statement that " $b$ is the $m$-cutting bond for $\boldsymbol{v}_{N-1} \stackrel{A}{\rightarrow} n$."

(ii) The second cutting bond for (5.4) is the $m$-cutting bond for $\boldsymbol{v}_{N-1} \stackrel{A}{\rightarrow} n$.

Note that, under $\widetilde{\mathbb{P}}_{N}$, the event that $b$ is an $m$-cutting bond implies that $b \neq\left(\boldsymbol{u}_{N}, \boldsymbol{v}_{N}\right)$, since $b$ must be occupied whereas $\left(\boldsymbol{u}_{N}, \boldsymbol{v}_{N}\right)$ is vacant.

Several definitions are required to formulate the result of the second expansion. Let

$$
\begin{aligned}
& H_{m}(\boldsymbol{v}, \boldsymbol{y} ; A)=\{\boldsymbol{v} \stackrel{A}{\rightarrow} \boldsymbol{y}\} \cap\{\nexists m \text {-cutting bond for } \boldsymbol{v} \stackrel{A}{\rightarrow} \boldsymbol{y}\}, \\
& H_{m, n}(\boldsymbol{v} ; A)=\{\boldsymbol{v} \stackrel{A}{\rightarrow} n\} \cap\{\nexists m \text {-cutting bond for } \boldsymbol{v} \stackrel{A}{\rightarrow} n\} .
\end{aligned}
$$

Recall our convention (4.15) that $\boldsymbol{v}_{-1}=\mathbf{0}$ and $\tilde{C}_{-1}=\{\boldsymbol{0}\}$. For $N \geqslant 0$, let

$$
\begin{aligned}
& e_{m, n}^{(N)}(4 ; A)=\widetilde{\mathbb{E}}_{N}\left(I\left[E^{\prime}\left(\boldsymbol{v}_{N-1}, \boldsymbol{u}_{N} ; \tilde{C}_{N-1}\right) \cap H_{m, n}\left(\boldsymbol{v}_{N-1} ; A\right)\right] \Pi_{m}\left(\boldsymbol{v}_{N} ; \tilde{C}_{N}\right)\right), \\
& e_{m, n}^{(N)}(5 ; A)=-\sum_{b \neq\left(\boldsymbol{u}_{N}, \boldsymbol{v}_{N}\right)} J_{\underline{b}, \bar{b}} \widetilde{\mathbb{E}}_{N}\left(I\left[E^{\prime}\left(\boldsymbol{v}_{N-1}, \boldsymbol{u}_{N} ; \tilde{C}_{N-1}\right) \cap H_{m}\left(\boldsymbol{v}_{N-1}, \underline{b} ; A\right)\right] \chi_{n}\left(\bar{b} ; \tilde{C}_{N}^{b}\right) \Pi_{m}\left(\boldsymbol{v}_{N} ; \tilde{C}_{N}\right)\right),
\end{aligned}
$$

where $\tilde{C}_{N}^{b}=\tilde{C}_{N}^{b}\left(\boldsymbol{v}_{N-1}\right)$. For $j=4,5$ and $N \geqslant 1$, let

$$
\begin{aligned}
& d_{m_{1}, n}^{(0)}(j ; A)=\sum_{\left(\boldsymbol{u}_{0}, \boldsymbol{v}_{0}\right)} J_{\boldsymbol{u}_{0}, \boldsymbol{v}_{0}} e_{m_{1}, n}^{(0)}(j ; A), \\
& d_{m_{1}, n}^{(N)}(j ; A)=\sum_{\left(\boldsymbol{u}_{N-1}, \boldsymbol{v}_{N-1}\right)} J_{\boldsymbol{u}_{N-1}, \boldsymbol{v}_{N-1}} \sum_{\left(\boldsymbol{u}_{N}, \boldsymbol{v}_{N}\right)} J_{\boldsymbol{u}_{N}, \boldsymbol{v}_{N}} M_{\mathbf{0}, \boldsymbol{u}_{N-1} ;\{\mathbf{0}\}}^{(N)}\left(e_{m_{1}, n}^{(N)}(j ; A)\right) .
\end{aligned}
$$

For $N \geqslant 1$, we also define

$$
\begin{aligned}
& \tilde{M}_{\left(\boldsymbol{u}_{0}, \boldsymbol{v}_{0}\right)}^{(1)}(X)=\widetilde{\mathbb{E}}_{0}\left(I\left[\left\{\boldsymbol{0} \Rightarrow \boldsymbol{u}_{0}\right\}\right] X\right), \\
& \tilde{M}_{\left(\boldsymbol{u}_{N}, \boldsymbol{v}_{N}\right)}^{(N+1)}(X)=\sum_{\left(\boldsymbol{u}_{N-1}, \boldsymbol{v}_{N-1}\right)} J_{\boldsymbol{u}_{N-1}, \boldsymbol{v}_{N-1}} M_{\mathbf{0}, \boldsymbol{u}_{N-1} ;\{\boldsymbol{0}\}}^{(N)}\left(\widetilde{\mathbb{E}}_{N}\left(I\left[E^{\prime}\left(\boldsymbol{v}_{N-1}, \boldsymbol{u}_{N} ; \tilde{C}_{N-1}\right)\right] X\right)\right) .
\end{aligned}
$$

Finally, for $N \geqslant 0$ we define

$$
\phi_{m_{1}, m_{2}}^{(N)}(A)=\sum_{\left(\boldsymbol{u}_{N}, \boldsymbol{v}_{N}\right)} J_{\boldsymbol{u}_{N}, \boldsymbol{v}_{N}} \sum_{b \neq\left(\boldsymbol{u}_{N}, \boldsymbol{v}_{N}\right)} J_{\underline{b}, \bar{b}} \tilde{M}_{\left(\boldsymbol{u}_{N}, \boldsymbol{v}_{N}\right)}^{(N+1)}\left(I\left[H_{m_{1}}\left(\boldsymbol{v}_{N-1}, \underline{b} ; A\right)\right] \Pi_{m_{2}}\left(\bar{b} ; \tilde{C}_{N}^{b}\right) \Pi_{m_{1}}\left(\boldsymbol{v}_{N} ; \tilde{C}_{N}\right)\right) .
$$

Now we are ready to state the result of the second expansion. 
Proposition 5.2 (The second expansion for $\chi_{n}$ ). For $n, m \geqslant 0, N \geqslant 0$ and $A \subseteq \Lambda$,

$$
\kappa_{m, n}^{(N)}(A)=\sum_{m_{2}=m}^{n} \phi_{m, m_{2}}^{(N)}(A) \theta_{n-m_{2}}-\left[d_{m, n}^{(N)}(4 ; A)+d_{m, n}^{(N)}(5 ; A)\right] .
$$

The proof of Proposition 5.2 is given below in Section 5.3. Let

$$
\begin{aligned}
& d_{m, n}^{(N)}(A)=d_{m, n}^{(N)}(4 ; A)+d_{m, n}^{(N)}(5 ; A), \\
& d_{m, n}^{(N)}=-\frac{1}{2} d_{m, n}^{(N)}\left(\tilde{C}_{N-1}\right)+d_{m, n}^{(N)}\left(\left\{\boldsymbol{v}_{N-1}\right\}\right),
\end{aligned}
$$

and

$$
\phi_{m_{1}, m_{2}}^{(N)}=-\frac{1}{2} \phi_{m_{1}, m_{2}}^{(N)}\left(\tilde{C}_{N-1}\right)+\phi_{m_{1}, m_{2}}^{(N)}\left(\left\{\boldsymbol{v}_{N-1}\right\}\right) .
$$

Then (5.1) follows immediately from Proposition 5.2 and (4.29). As noted below (5.1), this in turn completes the proof of Theorem 1.1(i).

For $j=4,5$, we define

$$
e_{n}^{(N)}(j)=\sum_{m_{1}=1}^{\lfloor n / 2\rfloor}\left[-\frac{1}{2} d_{m_{1}, n}^{(N)}\left(j ; \tilde{C}_{N-1}\right)+d_{m_{1}, n}^{(N)}\left(j ;\left\{\boldsymbol{v}_{N-1}\right\}\right)\right] \theta_{n-m_{1}} .
$$

Then (5.2) can be rewritten as

$$
e_{n}^{(N)}=e_{n}^{(N)}(1)+e_{n}^{(N)}(2)+e_{n}^{(N)}(3)+e_{n}^{(N)}(4)+e_{n}^{(N)}(5) .
$$

Our proof of (1.19) in Section 8 is based on the decomposition (5.19).

\subsection{Identification of $\phi_{1,1}$}

We now prove the first statement of Theorem 1.1(ii), namely that $\phi_{1,1}=\frac{1}{2} p_{c}^{2} \sum_{x} D(x)(1-D(x))$. By (1.4), (1.7) and (1.9), it then follows that $\phi_{1,1}=\frac{1}{2}+\mathrm{O}(\beta)$. According to (5.13) and (4.15), $\phi_{1,1}^{(N)}(A)=0$ unless $N=0$. Also, by (5.17) and (4.15), $\phi_{1,1}=\frac{1}{2} \phi_{1,1}^{(0)}(\{\mathbf{0}\})$. Thus, it suffices to show that $\phi_{1,1}^{(0)}(\{\mathbf{0}\})=p_{c}^{2} \sum_{x} D(x)(1-D(x))$.

For this, we use (5.13). Note that $\Pi_{1}\left(\bar{b} ; \tilde{C}_{0}^{b}\right)=\delta_{m_{\bar{b}}, 1}$ and $\Pi_{1}\left(\boldsymbol{v}_{0} ; \tilde{C}_{0}\right)=\delta_{m_{v_{0}}, 1}$, by (4.7). Therefore, by (4.15), we have $H_{1}\left(\boldsymbol{v}_{N-1}, \underline{b} ;\{\mathbf{0}\}\right)=H_{1}(\mathbf{0}, \mathbf{0} ;\{\mathbf{0}\})$, which is the trivial event by (5.5). Also, since $m_{\boldsymbol{v}_{0}}=1, \boldsymbol{u}_{0}$ must be $\mathbf{0}$, so that $\left\{\boldsymbol{0} \Rightarrow \boldsymbol{u}_{0}\right\}$ in (5.11) is trivially satisfied. We conclude that

$$
\tilde{M}_{\left(\boldsymbol{u}_{0}, \boldsymbol{v}_{0}\right)}^{(1)}\left(I\left[H_{m_{1}}(\mathbf{0}, \underline{b} ;\{\mathbf{0}\})\right] \Pi_{1}\left(\bar{b} ; \tilde{C}_{0}^{b}\right) \Pi_{1}\left(\boldsymbol{v}_{0} ; \tilde{C}_{0}\right)\right)=1 .
$$

It then follows from (5.13) that

$$
\phi_{1,1}^{(0)}(\{\mathbf{0}\})=\sum_{\boldsymbol{v}_{0}} J_{\mathbf{0}, v_{0}} \sum_{\bar{b} \neq \boldsymbol{v}_{0}} J_{\mathbf{0}, \bar{b}}=p_{c}^{2} \sum_{x} D(x)(1-D(x)),
$$

as required.

\subsection{Proof of Proposition 5.2}

To simplify the notation, for $N \geqslant 0$, we write

$$
E_{N}^{\prime}=E^{\prime}\left(\boldsymbol{v}_{N-1}, \boldsymbol{u}_{N} ; \tilde{C}_{N-1}\right) .
$$

In particular, according to (3.23) and the convention (4.15), $E_{0}^{\prime}=\left\{\boldsymbol{0} \Rightarrow \boldsymbol{u}_{0}\right\}$. For the second expansion, the following proposition plays the role that was played for the first expansion by Proposition 4.3. 
Proposition 5.3 (The second cutting bond). For all $A \subseteq \Lambda, N \geqslant 0, m \geqslant 0, n \geqslant 1$, and for all bonds $b$,

$$
\begin{aligned}
& E_{N}^{\prime} \cap\left\{b \text { is m-cutting for } \boldsymbol{v}_{N-1} \stackrel{A}{\rightarrow} n\right\} \\
& \quad=\left\{E_{N}^{\prime} \cap H_{m}\left(\boldsymbol{v}_{N-1}, \underline{b} ; A\right) \text { in } \tilde{C}_{N}^{b}\left(\boldsymbol{v}_{N-1}\right)\right\} \cap\{b \text { occ. }\} \cap\left\{\bar{b} \rightarrow n \text { in } \Lambda \backslash \tilde{C}_{N}^{b}\left(\boldsymbol{v}_{N-1}\right)\right\} .
\end{aligned}
$$

Proof. By (3.2), it suffices to prove that

$$
\begin{aligned}
& E_{N}^{\prime} \cap\left\{b \text { is } m \text {-cutting for } \boldsymbol{v}_{N-1} \stackrel{A}{\rightarrow} n\right\} \\
& \quad=\left\{E_{N}^{\prime} \cap H_{m}\left(\boldsymbol{v}_{N-1}, \underline{b} ; A\right) \text { in } \tilde{C}_{N}^{b}\left(\boldsymbol{v}_{N-1}\right)\right\} \cap\left\{b \text { occ. and piv. for } \boldsymbol{v}_{N-1} \rightarrow n\right\} .
\end{aligned}
$$

Since $m_{\bar{b}} \geqslant m$, and since $E_{N}^{\prime}$ depends only on the occupation status of bonds below $m_{\boldsymbol{u}_{N}} \leqslant m-1$, we have

$$
E_{N}^{\prime}=\left\{E_{N}^{\prime} \text { in } \tilde{C}_{N}^{b}\left(\boldsymbol{v}_{N-1}\right)\right\} .
$$

Also, for $b$ such that $m_{\bar{b}} \geqslant m$, we have

$$
\begin{aligned}
\left\{b \text { is } m \text {-cutting for } \boldsymbol{v}_{N-1} \stackrel{A}{\rightarrow} n\right\}= & \left\{b \text { occ. and piv. for } \boldsymbol{v}_{N-1} \rightarrow n\right\} \cap\left\{\boldsymbol{v}_{N-1} \stackrel{A}{\rightarrow} \underline{b}\right\} \\
& \cap\left\{\nexists \text { previous } m \text {-cutting bond for } \boldsymbol{v}_{N-1} \stackrel{A}{\rightarrow} n\right\} .
\end{aligned}
$$

Since the pivotal bonds for $\boldsymbol{v}_{N-1} \rightarrow n$ are ordered, any previous $m$-cutting bond for $\boldsymbol{v}_{N-1} \stackrel{A}{\rightarrow} n$ must be pivotal for $\boldsymbol{v}_{N-1} \rightarrow \underline{b}$. Therefore,

$$
\begin{aligned}
& \left\{\nexists \text { previous } m \text {-cutting bond for } \boldsymbol{v}_{N-1} \stackrel{A}{\rightarrow} n\right\}=\left\{\nexists m \text {-cutting bond for } \boldsymbol{v}_{N-1} \stackrel{A}{\rightarrow} \underline{b}\right\} \\
& =\left\{\left\{\nexists m \text {-cutting bond for } \boldsymbol{v}_{N-1} \stackrel{A}{\rightarrow} \underline{b}\right\} \text { in } \tilde{C}_{N}^{b}\left(\boldsymbol{v}_{N-1}\right)\right\},
\end{aligned}
$$

where the last equality again follows from the orientation. Since

$$
\left\{\boldsymbol{v}_{N-1} \stackrel{A}{\rightarrow} \underline{b}\right\}=\left\{\boldsymbol{v}_{N-1} \stackrel{A}{\rightarrow} \underline{b} \text { in } \tilde{C}_{N}^{b}\left(\boldsymbol{v}_{N-1}\right)\right\},
$$

we conclude from (5.5) that

$$
\left\{\boldsymbol{v}_{N-1} \stackrel{A}{\rightarrow} \underline{b}\right\} \cap\left\{\nexists \text { previous } m \text {-cutting bond for } \boldsymbol{v}_{N-1} \stackrel{A}{\rightarrow} n\right\}=\left\{H_{m}\left(\boldsymbol{v}_{N-1}, \underline{b} ; A\right) \text { in } \tilde{C}_{N}^{b}\left(\boldsymbol{v}_{N-1}\right)\right\} .
$$

Then (5.24) follows from (2.3) and (5.29), and the proof is complete.

Proof of Proposition 5.2. We will show that

$$
\begin{aligned}
\widetilde{\mathbb{E}}_{N} & \left(I\left[E_{N}^{\prime} \cap\left\{\boldsymbol{v}_{N-1} \stackrel{A}{\rightarrow} n\right\}\right] \Pi_{m}\left(\boldsymbol{v}_{N} ; \tilde{C}_{N}\right)\right) \\
= & \sum_{m_{2}=m}^{n} \sum_{b \neq\left(\boldsymbol{u}_{N}, \boldsymbol{v}_{N}\right)} J_{\underline{b}, \bar{b}} \widetilde{\mathbb{E}}_{N}\left(I\left[E_{N}^{\prime} \cap H_{m}\left(\boldsymbol{v}_{N-1}, \underline{b} ; A\right)\right] \Pi_{m_{2}}\left(\bar{b} ; \tilde{C}_{N}^{b}\right) \Pi_{m}\left(\boldsymbol{v}_{N} ; \tilde{C}_{N}\right)\right) \theta_{n-m_{2}} \\
& +e_{m, n}^{(N)}(4 ; A)+e_{m, n}^{(N)}(5 ; A) .
\end{aligned}
$$

This suffices, since substitution of (5.30) into (5.4) gives

$$
\begin{aligned}
& \kappa_{m, n}^{(0)}\left(\boldsymbol{v}_{N-1} ; A, \tilde{C}_{N-1}\right) \\
& =\sum_{\left(\boldsymbol{u}_{N}, \boldsymbol{v}_{N}\right)} J_{\boldsymbol{u}_{N}, \boldsymbol{v}_{N}} \sum_{m_{2}=m}^{n} \sum_{b \neq\left(\boldsymbol{u}_{N}, \boldsymbol{v}_{N}\right)} J_{\underline{b}, \bar{b}} \widetilde{\mathbb{E}}_{N}\left(I\left[E_{N}^{\prime} \cap H_{m}\left(\boldsymbol{v}_{N-1}, \underline{b} ; A\right)\right] \Pi_{m_{2}}\left(\bar{b} ; \tilde{C}_{N}^{b}\right) \Pi_{m}\left(\boldsymbol{v}_{N} ; \tilde{C}_{N}\right)\right) \theta_{n-m_{2}} \\
& \quad+\sum_{\left(\boldsymbol{u}_{N}, \boldsymbol{v}_{N}\right)} J_{\boldsymbol{u}_{N}, \boldsymbol{v}_{N}}\left[e_{m, n}^{(N)}(4 ; A)+e_{m, n}^{(N)}(5 ; A)\right],
\end{aligned}
$$

and substitution of (5.31) into (4.13), together with (5.13) and (5.9), (5.10), gives the desired result (5.14).

To prove (5.30), we use the partition

$$
\left\{\boldsymbol{v}_{N-1} \stackrel{A}{\rightarrow} n\right\}=H_{m, n}\left(\boldsymbol{v}_{N-1} ; A\right) \dot{\cup} \bigcup_{b}^{\cdot}\left(\left\{b \text { is } m \text {-cutting for } \boldsymbol{v}_{N-1} \stackrel{A}{\rightarrow} n\right\}\right) .
$$


By (5.32) and (5.7),

$$
\begin{aligned}
& \widetilde{\mathbb{E}}_{N}\left(I\left[E_{N}^{\prime} \cap\left\{\boldsymbol{v}_{N-1} \stackrel{A}{\rightarrow} n\right\}\right] \Pi_{m}\left(\boldsymbol{v}_{N} ; \tilde{C}_{N}\right)\right) \\
& \quad=e_{m, n}^{(N)}(4 ; A)+\sum_{b} \widetilde{\mathbb{E}}_{N}\left(I\left[E_{N}^{\prime} \cap\left\{b \text { is } m \text {-cutting for } \boldsymbol{v}_{N-1} \stackrel{A}{\rightarrow} n\right\}\right] \Pi_{m}\left(\boldsymbol{v}_{N} ; \tilde{C}_{N}\right)\right) .
\end{aligned}
$$

Let

$$
\tilde{C}_{N, m}=\left\{\boldsymbol{x} \in \tilde{C}_{N}: m_{x} \leqslant m\right\} .
$$

Then

$$
\Pi_{m}\left(\boldsymbol{v}_{N} ; \tilde{C}_{N}\right)=\Pi_{m}\left(\boldsymbol{v}_{N} ; \tilde{C}_{N, m-1}\right),
$$

since the first term in (4.7) does not depend on $\tilde{C}_{N}$, while the second only depends on $\tilde{C}_{N}$ up to time $m-1$. Conditioning on the set $\tilde{C}_{N, m-1}$ then gives

$$
\begin{aligned}
& \widetilde{\mathbb{E}}_{N}\left(I\left[E_{N}^{\prime} \cap\left\{\boldsymbol{v}_{N-1} \stackrel{A}{\rightarrow} n\right\}\right] \Pi_{m}\left(\boldsymbol{v}_{N} ; \tilde{C}_{N}\right)\right) \\
& \quad=e_{m, n}^{(N)}(4 ; A)+\sum_{b} \sum_{C} \Pi_{m}\left(\boldsymbol{v}_{N} ; C\right) \tilde{\mathbb{P}}_{N}\left(E_{N}^{\prime} \cap\left\{b \text { is } m \text {-cutting for } \boldsymbol{v}_{N-1} \stackrel{A}{\rightarrow} n\right\} \cap\left\{\tilde{C}_{N, m-1}=C\right\}\right) .
\end{aligned}
$$

We again write $\tilde{C}_{N}^{b}=\tilde{C}_{N}^{b}\left(\boldsymbol{v}_{N-1}\right)$. Since $m \leqslant m_{\bar{b}}$,

$$
\left\{\tilde{C}_{N, m-1}=C\right\}=\left\{\left\{\tilde{C}_{N, m-1}=C\right\} \text { in } \tilde{C}_{N}^{b}\right\} .
$$

By Proposition 5.3 and the independence stated in Lemma 2.2, for $b \neq\left(\boldsymbol{u}_{N}, \boldsymbol{v}_{N}\right)$, it follows that

$$
\begin{aligned}
& \tilde{\mathbb{P}}_{N}\left(E_{N}^{\prime} \cap\left\{b \text { is } m \text {-cutting for } \boldsymbol{v}_{N-1} \stackrel{A}{\rightarrow} n\right\} \cap\left\{\tilde{C}_{N, m-1}=C\right\}\right) \\
& \quad=J_{\underline{b}, \bar{b}} \tilde{\mathbb{P}}_{N}\left(\left\{E_{N}^{\prime} \cap H_{m}\left(\boldsymbol{v}_{N-1}, \underline{b} ; A\right) \cap\left\{\tilde{C}_{N, m-1}=C\right\} \text { in } \tilde{C}_{N}^{b}\right\} \cap\left\{\bar{b} \rightarrow n \text { in } \Lambda \backslash \tilde{C}_{N}^{b}\right\}\right) .
\end{aligned}
$$

(Note that, according to the comment below Definition 5.1, the left-hand side of (5.38) is zero if $b=\left(\boldsymbol{u}_{N}, \boldsymbol{v}_{N}\right)$, but the right-hand side need not be zero.) Next we apply Lemma 2.2 to the right-hand side to obtain, for $b \neq\left(\boldsymbol{u}_{N}, \boldsymbol{v}_{N}\right)$,

$$
\begin{aligned}
& \tilde{\mathbb{P}}_{N}\left(E_{N}^{\prime} \cap\left\{b \text { is } m \text {-cutting for } \boldsymbol{v}_{N-1} \stackrel{A}{\rightarrow} n\right\} \cap\left\{\tilde{C}_{N, m-1}=C\right\}\right) \\
& \quad=J_{\underline{b}, \bar{b}} \widetilde{\mathbb{E}}_{N}\left(I\left[E_{N}^{\prime} \cap H_{m}\left(\boldsymbol{v}_{N-1}, \underline{b} ; A\right) \cap\left\{\tilde{C}_{N, m-1}=C\right\}\right]\left(\theta_{n}(\bar{b})-\mathbb{P}_{N, 1}\left(\bar{b} \stackrel{\tilde{C}_{N}^{b}}{\rightarrow} n\right)\right)\right),
\end{aligned}
$$

where we have used (3.17) and the fact that

$$
\left\{E_{N}^{\prime} \cap H_{m}\left(\boldsymbol{v}_{N-1}, \underline{b} ; A\right) \cap\left\{\tilde{C}_{N, m-1}=C\right\} \text { in } \tilde{C}_{N}^{b}\right\}=E_{N}^{\prime} \cap H_{m}\left(\boldsymbol{v}_{N-1}, \underline{b} ; A\right) \cap\left\{\tilde{C}_{N, m-1}=C\right\},
$$

again due to the orientation. We conclude from (5.36) and (5.39) that

$$
\begin{aligned}
\widetilde{\mathbb{E}}_{N} & \left(I\left[E_{N}^{\prime} \cap\left\{\boldsymbol{v}_{N-1} \stackrel{A}{\rightarrow} n\right\}\right] \Pi_{m}\left(\boldsymbol{v}_{N} ; \tilde{C}_{N}\right)\right)-e_{m, n}^{(N)}(4 ; A) \\
& =\sum_{C} \sum_{b \neq\left(\boldsymbol{u}_{N}, \boldsymbol{v}_{N}\right)} J_{\underline{b}, \bar{b}} \Pi_{m}\left(\boldsymbol{v}_{N} ; C\right) \widetilde{\mathbb{E}}_{N}\left(I\left[E_{N}^{\prime} \cap H_{m}\left(\boldsymbol{v}_{N-1}, \underline{b} ; A\right) \cap\left\{\tilde{C}_{N, m-1}=C\right\}\right]\left(\theta_{n}(\bar{b})-\mathbb{P}_{N, 1}\left(\bar{b} \stackrel{\tilde{C}_{N}^{b}}{\longrightarrow} n\right)\right)\right) \\
& =\sum_{b \neq\left(\boldsymbol{u}_{N}, \boldsymbol{v}_{N}\right)} J_{\underline{b}, \bar{b}} \widetilde{\mathbb{E}}_{N}\left(I\left[E_{N}^{\prime} \cap H_{m}\left(\boldsymbol{v}_{N-1}, \underline{b} ; A\right)\right] \Pi_{m}\left(\boldsymbol{v}_{N} ; \tilde{C}_{N, m-1}\right)\left(\theta_{n}(\bar{b})-\mathbb{P}_{N, 1}\left(\bar{b} \stackrel{\tilde{C}_{N}^{b}}{\longrightarrow} n\right)\right)\right)
\end{aligned}
$$

Finally, we rewrite $\mathbb{P}_{N, 1}\left(\bar{b} \stackrel{\tilde{C}_{N}^{b}}{\longrightarrow} n\right)$ using Proposition 3.4, as in (4.11). The subscript on $\mathbb{P}_{N, 1}$ indicates the arrival of a new oriented percolation model, coupled to $\mathbb{P}_{N}$ via the set $\tilde{C}_{N}^{b}$. With (5.8) and (5.35), this gives (5.30), and thus completes the proof. 


\section{Diagrammatic estimates: Bounds for $\pi$ and $\chi$}

In this section, we begin to set the stage for the proof of the bounds on $\phi_{m_{1}, m_{2}}$ and $e_{n+1}$ stated in Theorem 1.1(ii). In Section 6.1, we prove bounds on $\pi(\boldsymbol{v}, \boldsymbol{y} ; A)$, and in Section 6.2, we prove bounds on $\chi_{n}(\boldsymbol{v} ; A)$. These bounds are in terms of certain Feynman diagrams $P^{(N)}(\boldsymbol{v}, \boldsymbol{y} ; A)$, which are closely related to diagrams appearing in [14, Section 4]. These diagrams are defined recursively, which is natural given that $\pi^{(N)}$ is defined in terms of the recursively defined $M^{(N+1)}$ (see (3.25)). Later, the recursive nature of the diagrams will be instrumental in bounding the diagrams.

The discussion in this section applies for arbitrary $p$ and $d$, under the weak assumption on $D$.

\subsection{Bounds on $\pi$}

In this section, we obtain bounds on the function $\pi(\boldsymbol{v}, \boldsymbol{y} ; A)$ defined in (3.27). For the special case in which $\boldsymbol{v}=\mathbf{0}$ and $A=\{\mathbf{0}\}$, the function $\pi(\mathbf{0}, \boldsymbol{y} ;\{\mathbf{0}\})$ is identical to the function $\pi(\boldsymbol{y})$ of [14, Eq. (3.25)], apart from the minor change that we have $\pi(\mathbf{0})=1$ whereas [14] has $\pi(\mathbf{0})=0$ (recall the comment below (3.25)). It is proved in [14, Eq. (4.10)] and [14, Eq. (4.29)] that for $N \geqslant 0$ and $m \geqslant 0$,

$$
\pi_{m}^{(N)}(y) \leqslant P_{m}^{(N)}(y) \text {. }
$$

Here, $P_{m}^{(N)}(y)=P^{(N)}(\boldsymbol{y})$ (for $\boldsymbol{y}=(y, m)$ ) is a sum of diagrams which, for $p=p_{c}, d>4$ and $\beta$ sufficiently small, obey the bound

$$
\sum_{y} P_{m}^{(N)}(y) \leqslant \delta_{m, 0} \delta_{N, 0}+(C \beta)^{N \vee 1}(m+1)^{-d / 2} .
$$

Together, these estimates give (1.14).

Our goal now is to generalise (6.1) to $\pi(\boldsymbol{v}, \boldsymbol{y} ; A)$. The generalisation is in terms of Feynman diagrams $P^{(N)}(\boldsymbol{v}, \boldsymbol{y} ; A)$ which are defined as follows. We start with $N=0$, and, keeping the bond orientation in mind, define

$$
\begin{aligned}
& P^{(0)}(\boldsymbol{v}, \boldsymbol{y} ; \boldsymbol{a})=\sum_{\boldsymbol{t}} \tau(\boldsymbol{t}-\boldsymbol{v}) \tau(\boldsymbol{a}-\boldsymbol{t}) \tau(\boldsymbol{y}-\boldsymbol{a}) \tau(\boldsymbol{y}-\boldsymbol{t}), \\
& P^{(0)}(\boldsymbol{v}, \boldsymbol{y} ; A)=\sum_{\boldsymbol{a}} I[\boldsymbol{a} \in A] P^{(0)}(\boldsymbol{v}, \boldsymbol{y} ; \boldsymbol{a}) .
\end{aligned}
$$

We refer to the two-point functions appearing in the right-hand side of (6.3) as lines. In the diagram $P^{(0)}(\boldsymbol{v}, \boldsymbol{y} ; \boldsymbol{a})$, we declare the lines $\tau(\boldsymbol{t}-\boldsymbol{v})$ and $\tau(\boldsymbol{y}-\boldsymbol{t})$ appearing in (6.3) to be 0-admissible (below this is generalised to $N$-admissibility). We also define

$$
P(\boldsymbol{u}, \boldsymbol{z}, \boldsymbol{y})=\sum_{\boldsymbol{w}, \boldsymbol{v}} \tau(\boldsymbol{w}-\boldsymbol{z}) J_{\boldsymbol{u}, \boldsymbol{v}} P^{(0)}(\boldsymbol{v}, \boldsymbol{y} ; \boldsymbol{w}),
$$

where $J_{\boldsymbol{u}, \boldsymbol{v}}$ is given by (3.10), and where we continue to regard the lines $\tau(\boldsymbol{t}-\boldsymbol{v})$ and $\tau(\boldsymbol{y}-\boldsymbol{t})$ appearing in $P^{(0)}(\boldsymbol{v}, \boldsymbol{y} ; \boldsymbol{w})$ in (6.5) as 0-admissible. See Fig. 7 for depictions of (6.3) and (6.5).

\section{Definition 6.1.}

(i) Given a diagram and any line $\lambda$ of the diagram, Construction $1^{\lambda}(\boldsymbol{y})$ is the operation in which a new vertex $\boldsymbol{y}$ is inserted in line $\lambda$. Explicitly, this means that the two-point function $\tau(\boldsymbol{v}-\boldsymbol{u})$ corresponding to line $\lambda$ is replaced by $\tau(\boldsymbol{v}-\boldsymbol{y}) \tau(\boldsymbol{y}-\boldsymbol{u})$.

(ii) Given a diagram $F(\boldsymbol{v}, \boldsymbol{y})$ with two vertices carrying labels $\boldsymbol{v}, \boldsymbol{y}$ and any line $\lambda$ of the diagram, we write $F\left(\boldsymbol{v}, \boldsymbol{y} ; 1^{\lambda}(z)\right)$ for the diagram where Construction $1^{\lambda}(z)$ is performed to the diagram $F(\boldsymbol{v}, \boldsymbol{y})$.

(iii) Construction $1^{\lambda}(l)$ is the operation in which Construction $1^{\lambda}(y, l)$ is performed followed by summation over $y$ with $l$ fixed. Explicitly, this means that $\tau_{j-i}(v-u)$ corresponding to line $\lambda$ is replaced by

$$
\sum_{y} \tau_{j-l}(v-y) \tau_{l-i}(y-u)
$$

We also write Construction $1(l)$ for the operation in which Construction $1^{\lambda}(l)$ is performed, and then a sum over all lines $\lambda$ in the diagram is performed (resulting in a sum of diagrams). 

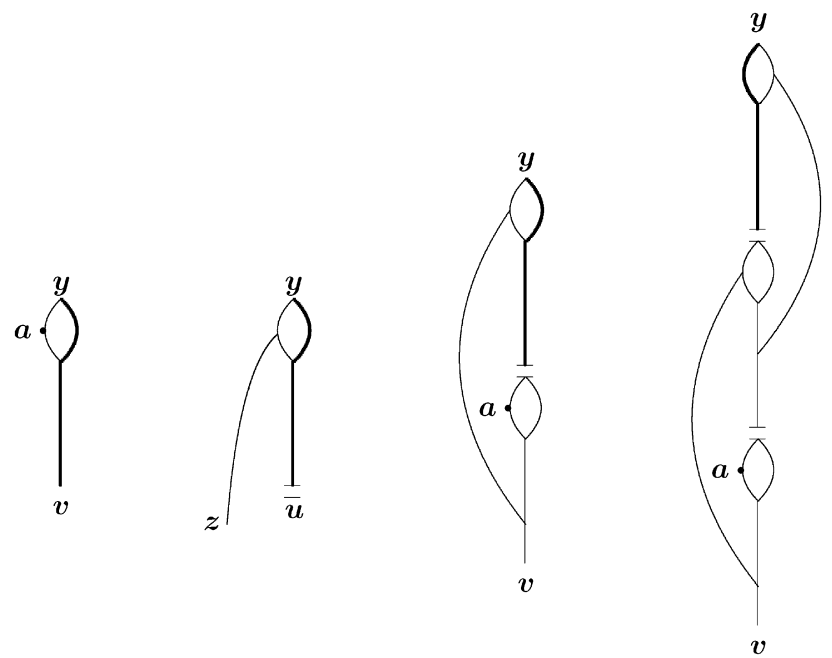

Fig. 7. Diagrams for $P^{(0)}(\boldsymbol{v}, \boldsymbol{y} ; \boldsymbol{a}), P(\boldsymbol{u}, \boldsymbol{z}, \boldsymbol{y})$, and examples of diagrams that contribute to $P^{(1)}(\boldsymbol{v}, \boldsymbol{y} ; \boldsymbol{a}), P^{(2)}(\boldsymbol{v}, \boldsymbol{y} ; \boldsymbol{a})$. The $N$-admissible lines are shown in bold.

For $N \geqslant 1$, given $P^{(N-1)}(\boldsymbol{v}, \boldsymbol{y} ; \boldsymbol{a})$ with its set of admissible lines, we define $P^{(N)}(\boldsymbol{v}, \boldsymbol{y} ; \boldsymbol{a})$ with its set of admissible lines recursively, as follows. First, let $P^{(N-1)}\left(\boldsymbol{v}, \boldsymbol{y} ; \boldsymbol{a}, 1^{\lambda}(\boldsymbol{z})\right)$ denote the result of applying Construction $1^{\lambda}(\boldsymbol{z})$ to $P^{(N-1)}(\boldsymbol{v}, \boldsymbol{y} ; \boldsymbol{a})$. Then, for $N \geqslant 1$, let

$$
P^{(N)}(\boldsymbol{v}, \boldsymbol{y} ; \boldsymbol{a})=\sum_{\lambda} \sum_{\boldsymbol{z}, \boldsymbol{w}} P^{(N-1)}\left(\boldsymbol{v}, \boldsymbol{w} ; \boldsymbol{a}, 1^{\lambda}(\boldsymbol{z})\right) P(\boldsymbol{w}, \boldsymbol{z}, \boldsymbol{y}),
$$

where the sum over $\lambda$ runs over the $(N-1)$-admissible lines, and where the $N$-admissible lines are by definition the 0 -admissible lines in the factor $P(\boldsymbol{w}, \boldsymbol{z}, \boldsymbol{y})$ appearing in (6.6). See Fig. 7.

We also define, for $N \geqslant 0$,

$$
P^{(N)}(\boldsymbol{v}, \boldsymbol{y} ; A)=\sum_{\boldsymbol{a}} I[\boldsymbol{a} \in A] P^{(N)}(\boldsymbol{v}, \boldsymbol{y} ; \boldsymbol{a}), \quad P^{(N)}(\boldsymbol{y})=P^{(N)}(\mathbf{0}, \boldsymbol{y} ;\{\boldsymbol{0}\}) .
$$

The $P^{(N)}(y)$ appearing in (6.7) is identical to the right-hand side of $(6.1)$, when $\boldsymbol{y}=(y, m)$. By (6.5), we can alternatively write, for $N \geqslant 1$,

$$
P^{(N)}(\boldsymbol{v}, \boldsymbol{y} ; A)=\sum_{\lambda} \sum_{z, \boldsymbol{w}} \sum_{\left(\boldsymbol{u}_{N-1}, \boldsymbol{v}_{N-1}\right)} J_{\boldsymbol{u}_{N-1}, \boldsymbol{v}_{N-1}} P^{(N-1)}\left(\boldsymbol{v}, \boldsymbol{u}_{N-1} ; A, 1^{\lambda}(\boldsymbol{z})\right) \tau(\boldsymbol{w}-\boldsymbol{z}) P^{(0)}\left(\boldsymbol{v}_{N-1}, \boldsymbol{y} ; \boldsymbol{w}\right) .
$$

An identical formula holds for $P^{(N)}(\boldsymbol{v}, \boldsymbol{y} ; \boldsymbol{a})$, where $A$ on both sides is replaced by $\boldsymbol{a}$.

Remark 6.2. When $P^{(M)}\left(\boldsymbol{v}, \boldsymbol{y} ; \boldsymbol{a}, 1^{\lambda}(\boldsymbol{z})\right)$ appears inside a sum over $\lambda$, our convention is that $\lambda$ is summed over the $M$-admissible lines.

The following proposition gives our main bound on $\pi^{(N)}(\boldsymbol{v}, \boldsymbol{y} ; A)$, and generalises (6.1).

Proposition 6.3. For $N \geqslant 0, \boldsymbol{v}, \boldsymbol{y} \in \Lambda$, and $A \subseteq \Lambda$,

$$
\pi^{(N)}(\boldsymbol{v}, \boldsymbol{y} ; A) \leqslant P^{(N)}(\boldsymbol{v}, \boldsymbol{y} ; A) .
$$

Proof. We will prove the two statements

$$
\begin{aligned}
& M_{\boldsymbol{v}, \boldsymbol{y} ; A}^{(N+1)}(1) \leqslant P^{(N)}(\boldsymbol{v}, \boldsymbol{y} ; A), \\
& M_{\boldsymbol{v}, \boldsymbol{y} ; A}^{(N+1)}\left(I\left[\boldsymbol{w} \in C_{N}\right]\right) \leqslant \sum_{\lambda} \sum_{z} P^{(N)}\left(\boldsymbol{v}, \boldsymbol{y} ; A, 1^{\lambda}(\boldsymbol{z})\right) \tau(\boldsymbol{w}-\boldsymbol{z}),
\end{aligned}
$$

simultaneously, using induction on $N$ (recall Remark 6.2). The first immediately gives (6.9), by (3.27). 
To verify (6.10) for $N=0$, we first recall (3.4) and observe that

$$
E^{\prime}(v, y ; A) \subseteq \mathcal{E}(v, y ; A) \equiv \bigcup_{a \in A, t \in \Lambda}(v \rightarrow t) \circ(t \rightarrow a) \circ(a \rightarrow y) \circ(t \rightarrow y),
$$

where, for events $F$ and $G, F \circ G$ denotes disjoint occurrence of $F$ and $G$. By the BK inequality (see [3]), this gives

$$
\begin{aligned}
M_{\boldsymbol{v}, \boldsymbol{y} ; A}^{(1)}(1) & =\mathbb{P}\left(E^{\prime}(\boldsymbol{v}, \boldsymbol{y} ; A)\right) \leqslant \sum_{\boldsymbol{a} \in A, \boldsymbol{t} \in \Lambda} \tau(\boldsymbol{t}-\boldsymbol{v}) \tau(\boldsymbol{a}-\boldsymbol{t}) \tau(\boldsymbol{y}-\boldsymbol{a}) \tau(\boldsymbol{y}-\boldsymbol{t}) \\
& =\sum_{\boldsymbol{a}} I[\boldsymbol{a} \in A] P^{(0)}(\boldsymbol{v}, \boldsymbol{y} ; \boldsymbol{a}),
\end{aligned}
$$

which is (6.10) for $N=0$.

To verify (6.11) for $N=0$, we use the standard fact (see [5, Lemma 2.5] or [15, Lemma 5.5.8] for details) that

$$
\begin{aligned}
& \mathbb{E}\left(I\left[E^{\prime}(\boldsymbol{v}, \boldsymbol{y} ; A)\right] I\left[\boldsymbol{w} \in C_{0}\right]\right) \\
& \quad \leqslant \sum_{\boldsymbol{a} \in A, \boldsymbol{z}, \boldsymbol{t} \in \Lambda} \tau(\boldsymbol{w}-\boldsymbol{z}) \tau(\boldsymbol{y}-\boldsymbol{a}) \tau(\boldsymbol{a}-\boldsymbol{t})(\tau(\boldsymbol{t}-\boldsymbol{v}) \tau(\boldsymbol{z}-\boldsymbol{t}) \tau(\boldsymbol{y}-\boldsymbol{z})+\tau(\boldsymbol{z}-\boldsymbol{v}) \tau(\boldsymbol{t}-\boldsymbol{z}) \tau(\boldsymbol{y}-\boldsymbol{t})) .
\end{aligned}
$$

The right-hand side of (6.14) is the same as the right-hand side of (6.11) for $N=0$, where the two terms in (6.14) correspond to the two terms in the sum over admissible lines in (6.11).

To advance the induction, we fix $N \geqslant 1$ and assume that (6.10), (6.11) hold for $N-1$. The recursion relation (3.35) implies that

$$
M_{\boldsymbol{v}, \boldsymbol{y} ; A}^{(N+1)}(1)=\sum_{\left(\boldsymbol{u}_{N-1}, \boldsymbol{v}_{N-1}\right)} J_{\boldsymbol{u}_{N-1}, \boldsymbol{v}_{N-1}} M_{\boldsymbol{v}, \boldsymbol{u}_{N-1} ; A}^{(N)}\left(M_{\boldsymbol{v}_{N-1}, \boldsymbol{y} ; \tilde{C}_{N-1}}^{(1)}(1)\right) .
$$

Application of (6.13) gives

$$
M_{\boldsymbol{v}, \boldsymbol{y} ; A}^{(N+1)}(1) \leqslant \sum_{\boldsymbol{w}} \sum_{\left(\boldsymbol{u}_{N-1}, \boldsymbol{v}_{N-1}\right)} J_{\boldsymbol{u}_{N-1}, \boldsymbol{v}_{N-1}} M_{\boldsymbol{v}, \boldsymbol{u}_{N-1} ; A}^{(N)}\left(I\left[\boldsymbol{w} \in \tilde{C}_{N-1}\right]\right) P^{(0)}\left(\boldsymbol{v}_{N-1}, \boldsymbol{y} ; \boldsymbol{w}\right) .
$$

We use (6.11) for $N-1$ to estimate the right-hand side (using $\tilde{C}_{N-1} \subseteq C_{N-1}$ ), and use (6.8) to complete the advancement of (6.10).

Similarly, for (6.11), we have

$$
M_{\boldsymbol{v}, \boldsymbol{y} ; A}^{(N+1)}\left(I\left[\boldsymbol{w} \in C_{N}\right]\right)=\sum_{\left(\boldsymbol{u}_{N-1}, \boldsymbol{v}_{N-1}\right)} J_{\boldsymbol{u}_{N-1}, \boldsymbol{v}_{N-1}} M_{\boldsymbol{v}, \boldsymbol{u}_{N-1} ; A}^{(N)}\left(M_{\boldsymbol{v}_{N-1}, \boldsymbol{y} ; \tilde{C}_{N-1}}^{(1)}\left(I\left[\boldsymbol{w} \in C_{N}\right]\right)\right) .
$$

Substitution of the bound (6.11) for $N=0$ (again using $\tilde{C}_{N-1} \subseteq C_{N-1}$ ) leads to

$$
\begin{aligned}
& M_{\boldsymbol{v}, \boldsymbol{y} ; A}^{(N+1)}\left(I\left[\boldsymbol{w} \in C_{N}\right]\right) \\
& \quad \leqslant \sum_{\lambda} \sum_{\boldsymbol{z}, \boldsymbol{w}^{\prime}} \sum_{\left.\boldsymbol{u}_{N-1}, \boldsymbol{v}_{N-1}\right)} J_{\boldsymbol{u}_{N-1}, \boldsymbol{v}_{N-1}} M_{\boldsymbol{v}, \boldsymbol{u}_{N-1} ; A}^{(N)}\left(I\left[\boldsymbol{w}^{\prime} \in C_{N-1}\right]\right) P^{(0)}\left(\boldsymbol{v}_{N-1}, \boldsymbol{y} ; \boldsymbol{w}^{\prime}, 1^{\lambda}(\boldsymbol{z})\right) \tau(\boldsymbol{w}-\boldsymbol{z}),
\end{aligned}
$$

where the sum over $\lambda$ runs over the 0 -admissible lines for $P^{(0)}\left(\boldsymbol{v}_{N-1}, \boldsymbol{y} ; \boldsymbol{w}^{\prime}\right)$ (recall Remark 6.2). We use the induction hypothesis (6.11) for $N-1$ to bound $M_{\boldsymbol{v}, \boldsymbol{u}_{N-1} ; A}^{(N)}\left(I\left[\boldsymbol{w}^{\prime} \in C_{N-1}\right]\right)$, and then rewrite the resulting bound noting that by (6.8),

$$
\begin{aligned}
& P^{(N)}\left(\boldsymbol{v}, \boldsymbol{y} ; A, 1^{\lambda}(\boldsymbol{z})\right) \\
& \quad=\sum_{\lambda^{\prime}} \sum_{\boldsymbol{z}^{\prime}, \boldsymbol{w}^{\prime}} \sum_{\left(\boldsymbol{u}_{N-1}, \boldsymbol{v}_{N-1}\right)} J_{\boldsymbol{u}_{N-1}, \boldsymbol{v}_{N-1}} P^{(N-1)}\left(\boldsymbol{v}, \boldsymbol{u}_{N-1} ; A, 1^{\lambda^{\prime}}\left(\boldsymbol{z}^{\prime}\right)\right) \tau\left(\boldsymbol{w}^{\prime}-\boldsymbol{z}^{\prime}\right) P^{(0)}\left(\boldsymbol{v}_{N-1}, \boldsymbol{y} ; \boldsymbol{w}^{\prime}, 1^{\lambda}(\boldsymbol{z})\right),
\end{aligned}
$$

where the sum over $\lambda^{\prime}$ runs over the $(N-1)$-admissible lines (recall Remark 6.2). This leads to

$$
M_{\boldsymbol{v}, \boldsymbol{y} ; A}^{(N+1)}\left(I\left[\boldsymbol{w} \in C_{N}\right]\right) \leqslant \sum_{\lambda} \sum_{z} P^{(N)}\left(\boldsymbol{v}, \boldsymbol{y} ; A, 1^{\lambda}(\boldsymbol{z})\right) \tau(\boldsymbol{w}-\boldsymbol{z}),
$$


which completes the advancement of (6.11).

Later, we will use the recursion formula, for $M \geqslant 1$ and $N \geqslant 0$,

$$
\sum_{\lambda} \sum_{\boldsymbol{w}, \boldsymbol{a}} \sum_{\left(\boldsymbol{u}_{N}, \boldsymbol{v}_{N}\right)} J_{\boldsymbol{u}_{N}, \boldsymbol{v}_{N}} P^{(N)}\left(\boldsymbol{u}_{N} ; 1^{\lambda}(\boldsymbol{w})\right) \tau(\boldsymbol{a}-\boldsymbol{w}) P^{(M-1)}\left(\boldsymbol{v}_{N}, \boldsymbol{y} ; \boldsymbol{a}\right)=P^{(N+M)}(\boldsymbol{y}) .
$$

To prove (6.21), we apply induction on $M$. For $M=1$, the claim follows from (6.8). To advance the induction, we note from (6.7), (6.8) that

$$
P^{(N+M)}(\boldsymbol{y})=\sum_{\lambda^{\prime}} \sum_{z, \boldsymbol{t}} \sum_{(\boldsymbol{u}, \boldsymbol{v})} J_{\boldsymbol{u}, \boldsymbol{v}} P^{(0)}(\boldsymbol{v}, \boldsymbol{y} ; \boldsymbol{t}) P^{(N+M-1)}\left(\boldsymbol{u} ; 1^{\lambda^{\prime}}(\boldsymbol{z})\right) \tau(\boldsymbol{t}-\boldsymbol{z}) .
$$

An application of the induction hypothesis yields that

$$
P^{(N+M-1)}\left(\boldsymbol{u} ; 1^{\lambda^{\prime}}(\boldsymbol{z})\right)=\sum_{\lambda} \sum_{\boldsymbol{w}, \boldsymbol{a}} \sum_{\left(\boldsymbol{u}_{N}, \boldsymbol{v}_{N}\right)} J_{\boldsymbol{u}_{N}, \boldsymbol{v}_{N}} P^{(N)}\left(\boldsymbol{u}_{N} ; 1^{\lambda}(\boldsymbol{w})\right) \tau(\boldsymbol{a}-\boldsymbol{w}) P^{(M-2)}\left(\boldsymbol{v}_{N}, \boldsymbol{u} ; \boldsymbol{a}, 1^{\lambda^{\prime}}(\boldsymbol{z})\right) .
$$

We substitute (6.23) into (6.22), and use the fact that

$$
\sum_{\lambda^{\prime}} \sum_{\boldsymbol{z}, \boldsymbol{t}} \sum_{(\boldsymbol{u}, \boldsymbol{v})} J_{\boldsymbol{u}, \boldsymbol{v}} P^{(0)}(\boldsymbol{v}, \boldsymbol{y} ; \boldsymbol{t}) P^{(M-2)}\left(\boldsymbol{v}_{N}, \boldsymbol{u} ; \boldsymbol{a}, 1^{\lambda^{\prime}}(\boldsymbol{z})\right) \tau(\boldsymbol{t}-\boldsymbol{z})=P^{(M-1)}\left(\boldsymbol{v}_{N}, \boldsymbol{y} ; \boldsymbol{a}\right)
$$

This then advances the induction and proves the claim in (6.21).

\subsection{Bounds on $\chi_{n}$}

In this section, we prove bounds on $\chi_{n}$. The proof will use the following lemmas and definition. Recall the definitions of $F_{n}^{\prime}(\boldsymbol{v} ; A)$ and $\mathcal{P}_{A}$ in (3.5) and (4.20).

Lemma 6.4. For $A \subseteq \Lambda, v \in \Lambda$, and $n \geqslant 0$,

$$
F_{n}^{\prime}(\boldsymbol{v} ; A) \subseteq \bigcup_{\boldsymbol{y} \in \Lambda} E^{\prime}(\boldsymbol{v}, \boldsymbol{y} ; A) \cap((\boldsymbol{v} \rightarrow n) \circ(\boldsymbol{y} \rightarrow n)) .
$$

Proof. Suppose that $F_{n}^{\prime}(\boldsymbol{v} ; A)$ occurs. When $\mathcal{P}_{A}=\varnothing$, the claim follows from Proposition 4.6, which says that then there exists $x$ for which $E^{\prime}(\boldsymbol{v},(x, n) ; A)=E^{\prime}(\boldsymbol{v},(x, n) ; A) \cap((\boldsymbol{v} \rightarrow n) \circ((x, n) \rightarrow n))$ holds, and this is a subset of the event on the right-hand side of (6.25). Thus, we are left to prove that, for every bond $b,\left\{b \in \mathcal{P}_{A}\right\} \cap F_{n}^{\prime}(\boldsymbol{v} ; A)$ is a subset of the right-hand side of (6.25). For this we use Proposition 4.3 to see that this event is a subset of the event in the right-hand side of (6.25) with $\boldsymbol{y}=\underline{b}$.

The following definition introduces a construction that adds a connection to $n$ in a diagram.

\section{Definition 6.5.}

(a) Given a diagram $F(\boldsymbol{u})$ with a vertex carrying label $\boldsymbol{u}$, Construction $\theta_{n}(\boldsymbol{u})$ is the diagram obtained by multiplying $F(\boldsymbol{u})$ by $\theta_{n-m_{\boldsymbol{u}}}$.

(b) Given a diagram, the result of applying Construction $\theta_{n}$ is the diagram obtained by Construction $1^{\lambda}(\boldsymbol{u})$ followed by Construction $\theta_{n}(\boldsymbol{u})$ and a summation over $\boldsymbol{u}$ and over all lines $\lambda$ in the diagram. Explicitly, this means that the two-point function $\tau(\boldsymbol{v}-\boldsymbol{w})$ associated with line $\lambda$ is replaced by $\sum_{\boldsymbol{u}} \tau(\boldsymbol{v}-\boldsymbol{u}) \tau(\boldsymbol{u}-\boldsymbol{w}) \theta_{n}(\boldsymbol{u})$, followed by a sum over $\lambda$.

We write $P^{(N)}\left(\boldsymbol{v}, \boldsymbol{y} ; \boldsymbol{a}, \theta_{n}\right)$ for the result of an application of Construction $\theta_{n}$ to $P^{(N)}(\boldsymbol{v}, \boldsymbol{y} ; \boldsymbol{a})$, and

$$
P^{(N)}\left(\boldsymbol{v}, \boldsymbol{y} ; A, \theta_{n}\right)=\sum_{\boldsymbol{a}} I[\boldsymbol{a} \in A] P^{(N)}\left(\boldsymbol{v}, \boldsymbol{y} ; \boldsymbol{a}, \theta_{n}\right)
$$


Lemma 6.6. For $A \subseteq \Lambda, N \geqslant 0, \boldsymbol{v}, \boldsymbol{u}_{N} \in \Lambda$ and $n \geqslant 0$,

$$
M_{\boldsymbol{v}, \boldsymbol{u}_{N} ; A}^{(N+1)}\left(I\left[\boldsymbol{v}_{N-1} \rightarrow n\right]\right) \leqslant P^{(N)}\left(\boldsymbol{v}, \boldsymbol{u}_{N} ; A, \theta_{n}\right),
$$

where we recall the abuse of notation above (4.15), and, for $N=0$, we set $\boldsymbol{v}_{-1}=\boldsymbol{v}$ and $\tilde{C}_{-1}=\{\boldsymbol{v}\}$.

Proof. This is a minor modification of the bound on $M_{\boldsymbol{v}, \boldsymbol{u}_{N} ; A}^{(N+1)}\left(I\left[\boldsymbol{w} \in \tilde{C}_{N}\right]\right)$ in (6.11), using the inequality

$$
\mathbb{P}_{N}\left(E^{\prime}\left(\boldsymbol{v}_{N-1}, \boldsymbol{u}_{N} ; \tilde{C}_{N-1}\right) \cap\left\{\boldsymbol{v}_{N-1} \rightarrow n\right\}\right) \leqslant P^{(0)}\left(\boldsymbol{v}_{N-1}, \boldsymbol{u}_{N} ; \tilde{C}_{N-1}, \theta_{n}\right)
$$

instead of (6.14), and we omit the details.

The following proposition gives an upper bound for $\chi_{n}(\boldsymbol{v} ; A)$ that is written as a minimum. In its statement, we write $P^{(N)}(\boldsymbol{v}, \boldsymbol{y} ; A)=P_{m, k}^{(N)}(v, y ; A)$, when $\boldsymbol{v}=(v, m)$ and $\boldsymbol{y}=(y, k)$. Later we will make use of both alternatives in the minimum, depending on the index $k$ in (6.28).

Proposition 6.7. For $A \subseteq \Lambda, N \geqslant 0, \boldsymbol{v}=(v, m) \in \Lambda$ and $n \geqslant 0$,

$$
\left|\chi_{n}^{(N)}(\boldsymbol{v} ; A)\right| \leqslant \sum_{k=m}^{n}\left(\left(\sum_{y \in \mathbb{Z}^{d}} P_{m, k}^{(N)}\left(v, y ; A, \theta_{n}\right)\right) \wedge\left(\sum_{y \in \mathbb{Z}^{d}} P_{m, k}^{(N)}(v, y ; A)\right)\right)(1 \vee p) \theta_{n-k-1},
$$

where, by convention, we set $\theta_{-1}=1$.

Proof. By (3.29), $\chi_{n}^{(N)}(\boldsymbol{v} ; A)=\gamma_{n}^{(N)}(\boldsymbol{v} ; A)-\rho_{n}^{(N)}(\boldsymbol{v} ; A)$. Since these two terms have opposite sign, it suffices to prove that $\gamma_{n}^{(N)}(\boldsymbol{v} ; A)$ and $\rho_{n}^{(N)}(\boldsymbol{v} ; A)$ are each bounded above by the right-hand side of (6.28).

We start with $\rho_{n}^{(N)}(\boldsymbol{v} ; A)$, which is defined in (3.28). We write $\boldsymbol{v}_{N}=(z, k+1)$ and use

$$
\mathbb{P}_{N+1}\left(\boldsymbol{v}_{N} \rightarrow n \text { in } \Lambda \backslash \tilde{C}_{N}\right) \leqslant \theta_{n-k-1}
$$

and

$$
I\left[\left\{\boldsymbol{v}_{N-1} \rightarrow n\right\} \cap\left\{\boldsymbol{v}_{N-1} \notin \tilde{C}_{N}\right\} \text { in } \tilde{C}_{N}\right] \leqslant I\left[\boldsymbol{v}_{N-1} \rightarrow n\right] .
$$

Performing the sum over $\boldsymbol{v}_{N}$ in (3.28), we get (recall also (3.16))

$$
\rho_{n}^{(N)}(\boldsymbol{v} ; A) \leqslant \sum_{\boldsymbol{u}_{N}} M_{\boldsymbol{v}, \boldsymbol{u}_{N} ; A}^{(N+1)}\left(I\left[\boldsymbol{v}_{N-1} \rightarrow n\right]\right) p \theta_{n-m_{\boldsymbol{u}_{N}-1}} .
$$

One alternative in the upper bound then follows from (6.26). On the other hand, by (6.10),

$$
M_{\boldsymbol{v}, \boldsymbol{u}_{N} ; A}^{(N+1)}\left(I\left[\boldsymbol{v}_{N-1} \rightarrow n\right]\right) \leqslant M_{\boldsymbol{v}, \boldsymbol{u}_{N} ; A}^{(N+1)}(1) \leqslant P^{(N)}\left(\boldsymbol{v}, \boldsymbol{u}_{N} ; A\right),
$$

which gives the other alternative and completes the proof for $\rho_{n}^{(N)}(\boldsymbol{v} ; A)$.

We proceed with $\gamma_{n}^{(N)}(\boldsymbol{v} ; A)$, which is defined in (3.26). Since

$$
\bigcup_{\boldsymbol{y} \in \Lambda} E^{\prime}(\boldsymbol{v}, \boldsymbol{y} ; A) \cap((\boldsymbol{v} \rightarrow n) \circ(\boldsymbol{y} \rightarrow n))=\bigcup_{\boldsymbol{y} \in \Lambda}\left(E^{\prime}(\boldsymbol{v}, \boldsymbol{y} ; A) \cap(\boldsymbol{v} \rightarrow n)\right) \circ(\boldsymbol{y} \rightarrow n),
$$

it follows from Lemma 6.4 and the BKR inequality (see [3, Theorem 2.19]) that

$$
\mathbb{P}\left(F_{n}^{\prime}(\boldsymbol{v} ; A)\right) \leqslant \sum_{\boldsymbol{y} \in \Lambda} \mathbb{P}\left(E^{\prime}(\boldsymbol{v}, \boldsymbol{y} ; A) \cap(\boldsymbol{v} \rightarrow n)\right) \theta_{n}(\boldsymbol{y}) .
$$

Substitution of the above bound into (3.26) yields (recall also (3.20) and (6.15))

$$
\begin{aligned}
\gamma_{n}^{(N)}(\boldsymbol{v} ; A) & =\sum_{\left(\boldsymbol{u}_{N-1}, \boldsymbol{v}_{N-1}\right)} J_{\boldsymbol{u}_{N-1}, \boldsymbol{v}_{N-1}} M_{\boldsymbol{v}, \boldsymbol{u}_{N-1} ; A}^{(N)}\left(\mathbb{P}_{N}\left(F_{n}^{\prime}\left(\boldsymbol{v}_{N-1} ; \tilde{C}_{N-1}\right)\right)\right) \\
& \leqslant \sum_{\boldsymbol{y} \in \Lambda} \sum_{\left(\boldsymbol{u}_{N-1}, \boldsymbol{v}_{N-1}\right)} J_{\boldsymbol{u}_{N-1}, \boldsymbol{v}_{N-1}} M_{\boldsymbol{v}, \boldsymbol{u}_{N-1} ; A}^{(N)}\left(\mathbb{P}_{N}\left(E^{\prime}\left(\boldsymbol{v}_{N-1}, \boldsymbol{y} ; \tilde{C}_{N-1}\right) \cap\left(\boldsymbol{v}_{N-1} \rightarrow n\right)\right)\right) \theta_{n}(\boldsymbol{y}) \\
& =\sum_{\boldsymbol{y} \in \Lambda} M_{\boldsymbol{v}, \boldsymbol{y} ; A}^{(N+1)}\left(I\left[\boldsymbol{v}_{N-1} \rightarrow n\right]\right) \theta_{n-m_{\boldsymbol{y}}} .
\end{aligned}
$$


The right-hand side of (6.35) is identical to the right-hand side of (6.31), apart from the fact that $\theta_{n-m_{y}}$ in (6.35) is replaced by $p \theta_{n-m_{y}-1}$ in (6.31). Since $\theta_{n-m_{y}} \leqslant \theta_{n-m_{y}-1}$ (using the convention below (6.28) when $m_{y}=n$ ), the desired estimate for $\gamma_{n}^{(N)}(\boldsymbol{v} ; A)$ follows. This completes the proof.

\section{Diagrammatic estimates: Bounds for $\phi$}

In this section, we prove the bound on $\phi_{m_{1}, m_{2}}$ stated in (1.18). We have already proved the statement above (1.18), that $\phi_{1,1}\left(p_{c}\right)=\frac{1}{2} p_{c}^{2} \sum_{x \in \mathbb{Z}^{d}} D(x)(1-D(x))$, in Section 5.2. Henceforth, we simplify the notation by writing

$$
M_{\boldsymbol{y}}^{(N)}(X)=M_{\mathbf{0}, \boldsymbol{y} ;\{\mathbf{0}\}}^{(N)}(X) .
$$

\subsection{The main estimate}

The following proposition gives the main estimate needed to prove (1.18). In its statement, we use the notation

$$
b_{m_{1}, m_{2}}=I\left[m_{1} \leqslant m_{2}\right]\left(m_{1}+1\right)^{-(d-2) / 2}\left(m_{2}-m_{1}+1\right)^{-(d-2) / 2} .
$$

Recall from (5.17) that

$$
\phi_{m_{1}, m_{2}}^{(N)}=\phi_{m_{1}, m_{2}}^{(N)}\left(\left\{\boldsymbol{v}_{N-1}\right\}\right)-\frac{1}{2} \phi_{m_{1}, m_{2}}^{(N)}\left(\tilde{C}_{N-1}\right),
$$

with $\phi_{m_{1}, m_{2}}^{(N)}(A)$ given by (5.13). Also, according to (4.3),

$$
\phi_{m_{1}, m_{2}}=\sum_{N=0}^{\infty}(-1)^{N} \phi_{m_{1}, m_{2}}^{(N)} .
$$

Proposition 7.1 (The bounds on $\left.\phi^{(N)}\right)$. Let $p=p_{c}, d>4$, for the spread-out model with $\beta$ sufficiently small. For $m_{2} \geqslant m_{1} \geqslant 1$ and $N \geqslant 1$,

$$
\begin{aligned}
& \left|\phi_{m_{1}, m_{2}}^{(N)}\left(\left\{\boldsymbol{v}_{N-1}\right\}\right)\right| \leqslant(C \beta)^{N} b_{m_{1}, m_{2}}, \\
& \left|\phi_{m_{1}, m_{2}}^{(N)}\left(\tilde{C}_{N-1}\right)\right| \leqslant(C \beta)^{N} b_{m_{1}, m_{2}} .
\end{aligned}
$$

Also, $\phi_{1,1}^{(N)}\left(\left\{\boldsymbol{v}_{N-1}\right\}\right)=\phi_{1,1}^{(N)}\left(\tilde{C}_{N-1}\right)=0$ for $N \geqslant 1$, and

$$
\phi_{m_{1}, m_{2}}^{(0)}\left(\left\{\boldsymbol{v}_{-1}\right\}\right)=\phi_{m_{1}, m_{2}}^{(0)}\left(\tilde{C}_{-1}\right)=\phi_{m_{1}, m_{2}}^{(0)}(\{\mathbf{0}\})= \begin{cases}p_{c}^{2} \sum_{x \in \mathbb{Z}^{d}} D(x)(1-D(x)) & \text { if }\left(m_{1}, m_{2}\right)=(1,1), \\ \mathrm{O}\left(\beta b_{m_{1}, m_{2}}\right) & \text { if }\left(m_{1}, m_{2}\right) \neq(1,1) .\end{cases}
$$

The case $\left(m_{1}, m_{2}\right)=(1,1)$ in $(7.7)$ has been proved already in Section 5.2. The remainder of Section 7 is devoted to the proof of Proposition 7.1. Before proceeding with the proof, we note that it implies (1.18).

Proof of (1.18). By (7.5)-(7.7), the contributions when $\left(m_{1}, m_{2}\right) \neq(1,1)$ sum up to $\mathrm{O}\left(\beta b_{m_{1}, m_{2}}\right)$. This is precisely the assertion of (1.18).

The proof of Proposition 7.1 consists of two main steps: (i) bounds on $\phi_{m_{1}, m_{2}}^{(N)}(A)$ by certain Feynman diagrams, and (ii) bounds on the Feynman diagrams. We describe these two steps in the next section, and show that they are sufficient to prove Proposition 7.1.

\subsection{Reduction of proof of the main estimate}

We carry out step (i) (mentioned above) in Section 7.2.1 and step (ii) in Section 7.2.2. In Section 7.2.3, we show that these steps are sufficient to prove Proposition 7.1 subject to Propositions 7.6, 7.7 below. The latter are proved in Sections 7.3, 7.4. 


\subsubsection{Diagrams for $\phi_{m_{1}, m_{2}}^{(N)}$}

The results of this section apply for general $p$ and $d$ under the weak assumption on $D$.

We will use several constructions to define the diagrams needed to bound $\phi_{m_{1}, m_{2}}^{(N)}$.

\section{Definition 7.2.}

(i) Given a diagram and any line $\lambda$ of the diagram, Construction $\ell^{\lambda}(y)$ is the operation in which a line to $y$ is inserted into line $\lambda$. Explicitly, this means that the two-point function $\tau(\boldsymbol{v}-\boldsymbol{u})$ corresponding to line $\lambda$ is replaced by

$$
\sum_{z} \tau(v-z) \tau(z-\boldsymbol{u}) \tau(\boldsymbol{y}-\boldsymbol{z})
$$

We omit the superscript $\lambda$, and write Construction $\ell(\boldsymbol{y})$, when we perform Construction $\ell^{\lambda}(\boldsymbol{y})$ followed by a sum over all possible lines $\lambda$. We write $F(\boldsymbol{v}, \boldsymbol{y} ; \ell(\boldsymbol{x}))$ for the diagram where Construction $\ell(\boldsymbol{x})$ is performed on the diagram $F(\boldsymbol{v}, \boldsymbol{y})$.

(ii) Similarly, for $\overrightarrow{\boldsymbol{y}}=\left(\boldsymbol{y}_{1}, \ldots, \boldsymbol{y}_{i}\right)$, Construction $\ell(\overrightarrow{\boldsymbol{y}})$ is the repeated application of Constructions $\ell\left(\boldsymbol{y}_{1}\right), \ldots, \ell\left(\boldsymbol{y}_{i}\right)$. Note that the order of application of the different Constructions $\ell\left(\boldsymbol{y}_{j}\right)$ is irrelevant.

For example, it follows directly from (6.11) that

$$
M_{\boldsymbol{y}}^{(N+1)}\left(I\left[\boldsymbol{a} \in \tilde{C}_{N}\right]\right) \leqslant \sum_{\lambda} P^{(N)}\left(\boldsymbol{y} ; \ell^{\lambda}(\boldsymbol{a})\right),
$$

where the sum over $\lambda$ runs over the $N$-admissible lines for $P^{(N)}(y)$ (recall Remark 6.2).

\section{Definition 7.3.}

(i) Given a diagram $F(\boldsymbol{u})$ with two vertices carrying labels $\mathbf{0}$ and $\boldsymbol{u}$ and with a certain set of admissible lines indexed by $\lambda$, Construction $2_{\boldsymbol{u}}^{(0)}(\boldsymbol{w})$ and $2_{\boldsymbol{u}}^{(1)}(\boldsymbol{w})$, applied to $F(\boldsymbol{u})$, respectively produce the diagrams

$$
\begin{aligned}
& \tilde{F}^{(0)}(\boldsymbol{w})=\sum_{\lambda} \sum_{\boldsymbol{u}, \boldsymbol{z}} F\left(\boldsymbol{u} ; 1^{\lambda}(\boldsymbol{z})\right) \tau(\boldsymbol{w}-\boldsymbol{u}) \tau(\boldsymbol{w}-\boldsymbol{z}), \\
& \tilde{F}^{(1)}(\boldsymbol{w})=\sum_{\lambda} \sum_{(\boldsymbol{u}, \boldsymbol{v}), \boldsymbol{z}} J_{\boldsymbol{u}, \boldsymbol{v}} F\left(\boldsymbol{u} ; 1^{\lambda}(\boldsymbol{z})\right) \tau(\boldsymbol{w}-\boldsymbol{z}) \tau(\boldsymbol{w}-\boldsymbol{v}),
\end{aligned}
$$

where the sum over $\lambda$ runs over the admissible lines.

(ii) Given a diagram $F(\boldsymbol{v}, \boldsymbol{y})$ with two vertices carrying labels $\boldsymbol{v}$ and $\boldsymbol{y}$ and with a certain set of admissible lines indexed by $\lambda$, Construction $E_{\boldsymbol{y}}(\boldsymbol{w})$ produces the diagram

$$
\tilde{F}(\boldsymbol{v}, \boldsymbol{w})=\sum_{\lambda} \sum_{\boldsymbol{y}, \boldsymbol{z}} F\left(\boldsymbol{v}, \boldsymbol{y} ; 1^{\lambda}(\boldsymbol{z})\right) P(\boldsymbol{u}, \boldsymbol{z}, \boldsymbol{w})=\sum_{\lambda} \sum_{\boldsymbol{a}} \sum_{\left(\boldsymbol{u}_{0}, \boldsymbol{v}_{0}\right)} J_{\boldsymbol{u}_{0}, \boldsymbol{v}_{0}} F\left(\boldsymbol{v}, \boldsymbol{u}_{0} ; \ell^{\lambda}(\boldsymbol{a})\right) P^{(0)}\left(\boldsymbol{v}_{0}, \boldsymbol{w} ; \boldsymbol{a}\right),
$$

where the sum over $\lambda$ runs over the admissible lines, and we recall (6.5).

Construction $E_{\boldsymbol{y}}(\boldsymbol{w})$ is the same as Construction $2_{\boldsymbol{y}}^{(1)}(\boldsymbol{w})$ followed by Construction $2_{\boldsymbol{w}}^{(0)}(\boldsymbol{y})$, where the unique admissible line prior to the application of Construction $2_{w}^{(0)}(y)$ is the line from $v$ to $\boldsymbol{w}$ added to the diagram in the application of Construction $2_{\boldsymbol{u}}^{(1)}(\boldsymbol{w})$ in (7.10).

Remark 7.4. By (6.6), the diagram $P^{(N)}(\boldsymbol{v}, \boldsymbol{y} ; A)$ is obtained by performing $N$ Constructions $E$ to the diagram $P^{(0)}(\boldsymbol{v}, \boldsymbol{y} ; A)$.

Definition 7.5. Given a diagram $F\left(\boldsymbol{y}_{1}\right)$ with two vertices carrying labels $\mathbf{0}$ and $\boldsymbol{y}_{1}$, Construction $V_{m}\left(\boldsymbol{y}_{2}\right)$ and Construction $\mathcal{E}_{m}\left(\boldsymbol{y}_{2}\right)$ produce the diagrams 


$$
\begin{aligned}
& F\left(\boldsymbol{y}_{1} ; V_{m}\left(\boldsymbol{y}_{2}\right)\right)=\sum_{\boldsymbol{v}: m_{\boldsymbol{v}}=m} F\left(\boldsymbol{y}_{1} ; \ell(\boldsymbol{v}), 1(\boldsymbol{z})\right) \tau\left(\boldsymbol{y}_{2}-\boldsymbol{v}\right) \tau\left(\boldsymbol{y}_{2}-\boldsymbol{z}\right), \\
& F\left(\boldsymbol{y}_{1} ; \mathcal{E}_{m}\left(\boldsymbol{y}_{2}\right)\right)=\sum_{\boldsymbol{z}} \sum_{\boldsymbol{a}: m_{\boldsymbol{a}} \geqslant m} F\left(\boldsymbol{y}_{1} ; 1(\boldsymbol{z}), \ell(\boldsymbol{a})\right) P^{(0)}\left(\boldsymbol{z}, \boldsymbol{y}_{2} ; \boldsymbol{a}\right) .
\end{aligned}
$$

Let

$$
\Pi_{m}^{(0)}(\boldsymbol{v} ; A)=\delta_{m, m_{v}}, \quad \Pi_{m}^{(M)}(\boldsymbol{v} ; A)=p \sum_{y \in \mathbb{Z}^{d}} \pi^{(M-1)}(\boldsymbol{v},(y, m-1) ; A),
$$

so that, by (4.7),

$$
\Pi_{m}(\boldsymbol{v} ; A)=\sum_{M=0}^{\infty}(-1)^{M} \Pi_{m}^{(M)}(\boldsymbol{v} ; A) .
$$

Denote by $\phi_{m_{1}, m_{2}}^{(N, K)}(A)$ the contribution to $\phi_{m_{1}, m_{2}}^{(N)}(A)$ in $(5.13)$ of $\Pi_{m_{1}}^{(M)}\left(\boldsymbol{v}_{N} ; \tilde{C}_{N}\right)$ and $\Pi_{m_{2}}^{(K)}\left(\bar{b} ; \tilde{C}_{N}^{b}\right)$. Then

$$
\left|\phi_{m_{1}, m_{2}}^{(N)}(A)\right| \leqslant \sum_{M, K=0}^{\infty} \phi_{m_{1}, m_{2}}^{(N, M)}(A) .
$$

The lowest order contributions to $\phi_{m_{1}, m_{2}}^{(N)}$, namely, $\phi_{m_{1}, m_{2}}^{(N, M, 0)}\left(\left\{\boldsymbol{v}_{N-1}\right\}\right)$ and $\phi_{m_{1}, m_{2}}^{(N, M, 0)}\left(\tilde{C}_{N-1}\right)$, are treated in the following proposition. For its statement, we define

$$
\begin{aligned}
& R^{(N)}\left(\boldsymbol{y}_{1}, \boldsymbol{y}_{2}\right)=P^{(N)}\left(\boldsymbol{y}_{1} ; V_{m_{y_{1}}}\left(\boldsymbol{y}_{2}\right)\right)=\sum_{z} \sum_{\boldsymbol{v}: m_{\boldsymbol{v}}=m_{y_{1}}} P^{(N)}\left(\boldsymbol{y}_{1} ; \ell(\boldsymbol{v}), 1(\boldsymbol{z})\right) \tau\left(\boldsymbol{y}_{2}-\boldsymbol{v}\right) \tau\left(\boldsymbol{y}_{2}-\boldsymbol{z}\right), \\
& Q^{(N)}\left(\boldsymbol{y}_{1}, \boldsymbol{y}_{2}\right)=P^{(N)}\left(\boldsymbol{y}_{1} ; \mathcal{E}_{m_{\boldsymbol{y}_{1}}}\left(\boldsymbol{y}_{2}\right)\right)=\sum_{\boldsymbol{z}} \sum_{\boldsymbol{a}: m_{\boldsymbol{a}}>m_{\boldsymbol{y}_{1}}} P^{(N)}\left(\boldsymbol{y}_{1} ; 1(\boldsymbol{z}), \ell(\boldsymbol{a})\right) P^{(0)}\left(\boldsymbol{z}, \boldsymbol{y}_{2} ; \boldsymbol{a}\right) .
\end{aligned}
$$

See Fig. 8.

We denote by $R_{m_{1}, m_{2}}^{(N, K)}\left(y_{1}, y_{2}\right)$ the result of $K$ applications of Construction $E$ to the second argument of $R_{m_{1}, l}^{(N+M)}\left(y_{1}, v\right)$ (thus, $R^{(N, M, K)}$ actually only depends on $N+M$ and $\left.K\right)$. Similarly, we denote by $Q_{m_{1}, m_{2}}^{(N, K)}\left(y_{1}, y_{2}\right)$ the result of $K$ applications of Construction $E$ to the second argument of $Q_{m_{1}, l}^{(N+M)}\left(y_{1}, v\right)$. The diagrammatic bounds for $\phi^{(N)}$ are given in the following proposition, whose proof is deferred to Section 7.4.

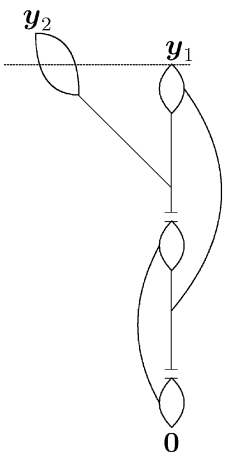

(a)

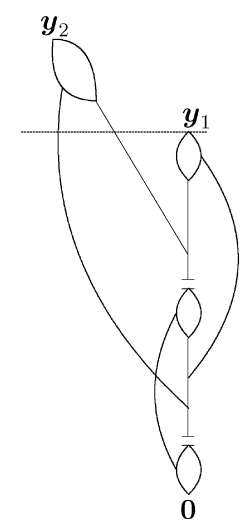

(b)

Fig. 8. Examples of diagrams that contribute to $R^{(2)}\left(\boldsymbol{y}_{1}, \boldsymbol{y}_{2}\right)$ and $Q^{(2)}\left(\boldsymbol{y}_{1}, \boldsymbol{y}_{2}\right)$. 
Proposition 7.6 (Diagrammatic bounds for $\left.\phi^{(N)}\right)$. Let $p$ and $d$ be arbitrary and assume the weak assumption on D. For $m_{2} \geqslant m_{1} \geqslant 1$ and $N, M, K \geqslant 0$,

$$
\phi_{m_{1}, m_{2}}^{(N, M, K)}\left(\left\{\boldsymbol{v}_{N-1}\right\}\right) \leqslant p^{2} \sum_{y_{1}, y_{2}} R_{m_{1}-1, m_{2}-1}^{(N, M, K)}\left(y_{1}, y_{2}\right) .
$$

For $m_{2} \geqslant m_{1} \geqslant 1$ and $N \geqslant 1, M, K \geqslant 0$,

$$
\phi_{m_{1}, m_{2}}^{(N, M, K)}\left(\tilde{C}_{N-1}\right) \leqslant p^{2} \sum_{y_{1}, y_{2}} R_{m_{1}-1, m_{2}-1}^{(N, M, K)}\left(y_{1}, y_{2}\right)+p^{2} \sum_{y_{1}, y_{2}} Q_{m_{1}-1, m_{2}-1}^{(N, M, K)}\left(y_{1}, y_{2}\right) .
$$

Also, for $p=p_{c}$ and $d>4$, for the spread-out model with $\beta$ sufficiently small, if $\left(m_{1}, m_{2}\right) \neq(1,1)$, then $\phi_{m_{1}, m_{2}}^{(0,0,0)}(\{\mathbf{0}\})=\mathrm{O}\left(\beta b_{m_{1}, m_{2}}\right)$.

\subsubsection{Bounds on diagrams for $\phi_{m_{1}, m_{2}}^{(N)}$}

The following proposition gives bounds on the Feynman diagrams that were used above to estimate $\phi_{m_{1}, m_{2}}$. Its proof is deferred to Section 7.3.

Proposition 7.7 (Bounds on $R^{(N, M, K)}$ and $Q^{(N, M, K)}$ ). Let $p=p_{c}$ and $d>4$, for the spread-out model with $\beta$ sufficiently small. For $m_{2} \geqslant m_{1} \geqslant 0$ and $N, M, K \geqslant 0$,

$$
\sum_{y_{1}, y_{2}} R_{m_{1}, m_{2}}^{(N, M)}\left(y_{1}, y_{2}\right) \leqslant C(C \beta)^{N+M+K} b_{m_{1}, m_{2}}
$$

For $m_{2} \geqslant m_{1} \geqslant 0$ and $N \geqslant 1, M, K \geqslant 0$,

$$
\sum_{y_{1}, y_{2}} Q_{m_{1}, m_{2}}^{(N, M)}\left(y_{1}, y_{2}\right) \leqslant(C \beta)^{N+M+K} b_{m_{1}, m_{2}} .
$$

\subsubsection{Proof of Proposition 7.1}

We now prove Proposition 7.1, subject to Propositions 7.6, 7.7. Let $p=p_{c}, d>4$, for the spread-out model with $\beta$ sufficiently small. Let $A$ denote either $\left\{\boldsymbol{v}_{N-1}\right\}$ or $\tilde{C}_{N-1}$. By (7.16) and Proposition 7.6,

$$
\left|\phi_{m_{1}, m_{2}}^{(N)}(A)\right| \leqslant p_{c}^{2} \sum_{M, K=0}^{\infty} \sum_{y_{1}, y_{2}} R_{m_{1}-1, m_{2}-1}^{(N, M, K)}\left(y_{1}, y_{2}\right)+p_{c}^{2} \sum_{M, K=0}^{\infty} \sum_{y_{1}, y_{2}} Q_{m_{1}-1, m_{2}-1}^{(N, M, K)}\left(y_{1}, y_{2}\right) .
$$

By Proposition 7.7, each of the sums on the right-hand side of (7.23) is at most $C(C \beta)^{N} b_{m_{1}-1, m_{2}-1}$. Since $b_{m_{1}-1, m_{2}-1} \leqslant C b_{m_{1}, m_{2}}$ for $m_{1}, m_{2} \geqslant 1$, this proves (7.5), (7.6).

The first two equalities in (7.7) follow from the convention (4.15). We have already noted in Section 5.2 that $\phi_{1,1}^{(0)}(\{\mathbf{0}\})=p_{c}^{2} \sum_{x} D(x)(1-D(x))$, and that $\phi_{1,1}^{(N)}(A)=0$ if $N \geqslant 1$, so it remains only to prove (7.7) when $\left(m_{1}, m_{2}\right) \neq$ $(1,1)$. For the remainder of the proof we assume that $\left(m_{1}, m_{2}\right) \neq(1,1)$. By $(7.21),(7.22)$, the contributions to $\phi_{m_{1}, m_{2}}^{(0, M)}(\{\boldsymbol{0}\})$ with $M+K \geqslant 1$ contain at least one factor $\beta$, so it suffices to prove that $\phi_{m_{1}, m_{2}}^{(0,0,0)}(\{\mathbf{0}\})=\mathrm{O}\left(\beta b_{m_{1}, m_{2}}\right)$. But this is the final statement of Proposition 7.6.

\subsection{Proof of Proposition 7.7}

In this section, we prove Proposition 7.7. We begin in Section 7.3.1 with some general techniques for extending bounds on simple diagrams to bounds on more complicated ones, and we use these techniques in Section 7.3.2 to prove the proposition.

\subsubsection{Convolution bounds}

In this section, we prove three lemmas which provide bounds on a diagram after a Construction has been performed on it, given a bound on the original diagram. In the proofs of the lemmas, we will use the following bounds on the two-point function, which follow from [14, Theorem 1.1]:

$$
\sum_{x} \tau_{m}(x) \leqslant K, \quad\left\|\tau_{m}\right\|_{\infty} \leqslant \begin{cases}K \beta(m+1)^{-d / 2} & (m \geqslant 1), \\ K(m+1)^{-d / 2} & (m \geqslant 0) .\end{cases}
$$


We use the first bound on $\left\|\tau_{m}\right\|_{\infty}$ when a line is guaranteed to have length $m \geqslant 1$, and use the second bound on $\left\|\tau_{m}\right\|_{\infty}$ otherwise.

We say that a diagram has at most $\mathcal{L}$ lines at any fixed time when, for every $m$, the number of factors $\tau(\boldsymbol{v}-\boldsymbol{u})$ with $m_{\boldsymbol{u}} \leqslant m$ and $m_{\boldsymbol{v}} \geqslant m$ is at most $\mathcal{L}$. For example, it is a simple consequence of the construction of $P^{(N)}(\boldsymbol{y})$ that it has at most 4 lines at any fixed time.

Lemma 7.8. Let $0 \leqslant l \leqslant k$ with $k \geqslant 1$, and assume (7.24). Let $G$ and $F_{k}(x)$ be diagrams such that $G$ and $\sum_{x} F_{k}(x)$ can be bounded by $B$ and $B(k+1)^{-d / 2}$, respectively, by associating $l^{1}$ - and $l^{\infty}$-norms to diagram lines and by using (7.24) to estimate these norms. Then the following statements hold.

(a) Application of Construction $1^{\lambda}(l)$ to $G$ produces a diagram that is bounded by $2^{(d+2) / 2} K B$, where $K$ is the constant of (7.24).

(b) Suppose that the diagram $F_{k}(u)$ has at most $\mathcal{L}$ lines at any fixed time, and that all diagram lines have time variables at most $k$. If $\sum_{u} F_{k}(u) \leqslant B \beta(k+1)^{-d / 2}$, then

$$
\sum_{x, u} F_{k}(u ; \ell(x, m)) \leqslant C \mathcal{L} B(k+1)^{-(d-2) / 2},
$$

with $C$ independent of $F, B, k, m$ and $\mathcal{L}$.

In each of (a), (b), the bound on the new diagram is obtained by associating $l^{1}$ - and $l^{\infty}$-norms to diagram lines and by using (7.24) to estimate these norms.

Proof. (a) This is [14, Lemma 4.6(a)].

(b) By definition,

$$
F_{k}(u ; \ell(x, m))=\sum_{(z, l)} F_{k}\left(u ; 1_{(z, l)}\right) \tau_{m-l}(x-z),
$$

and hence

$$
\sum_{x, u} F_{k}(u ; \ell(x, m))=\sum_{l=0}^{k}\left(\sum_{z, u} F_{k}\left(u ; 1_{(z, l)}\right)\right)\left(\sum_{x} \tau_{m-l}(x-z)\right) .
$$

The second factor is at most $K$, by (7.24). The first factor is bounded uniformly in $0 \leqslant l \leqslant k$ by $C \mathcal{L} B(k+1)^{-d / 2}$, by part (a). Finally, the sum over $l$ gives rise to a factor $k+1$.

Lemma 7.9. Let $d>4$ and $p \leqslant C$. Suppose that a diagram $F_{m_{1}, m_{2}}\left(y_{1}, y_{2}\right)$ satisfies the inequality

$$
\sum_{y_{1}, y_{2}} F_{m_{1}, m_{2}}\left(y_{1}, y_{2}\right) \leqslant C_{F} b_{m_{1}, m_{2}},
$$

with this bound obtained by associating $l^{1}$ - and $l^{\infty}$-norms to diagram lines and by using (7.24) to estimate these norms. Then application of Construction $2_{(v, k)}^{(i)}\left(y_{2}, m_{2}\right)$ to $F_{m_{1}, k}\left(y_{1}, v\right)$, followed by summation over $y_{1}$ and $y_{2}$, produces a diagram that is bounded by $C \beta^{i} C_{F} b_{m_{1}, m_{2}}$, with $C$ independent of $F$ and $C_{F}$. The bound on the new diagram is obtained by associating $l^{1}$ - and $l^{\infty}$-norms to diagram lines and by using (7.24) to estimate these norms. As a consequence, application of Construction $E$ to a diagram which obeys $\sum_{y_{1}, y_{2}} F_{m_{1}, m_{2}}\left(y_{1}, y_{2}\right) \leqslant C_{F} b_{m_{1}, m_{2}}$ produces a new diagram that is bounded by $C \beta C_{F} b_{m_{1}, m_{2}}$, with $C$ independent of $F$ and $C_{F}$.

Proof. For $m \geqslant 0$, let

$$
\tau_{m}^{(0)}(x)=\tau_{m}(x), \quad \tau_{m}^{(1)}(x)=\sum_{u} p_{c} D(u) \tau_{m-1}(x-u) I[m \geqslant 1] .
$$


Let $F_{m_{1}, m_{2}}^{(i)}\left(y_{1}, y_{2}\right)$ be the diagram obtained by application of Construction $2_{(v, k)}^{(i)}\left(y_{2}, m_{2}\right)$ to the diagram $F_{m_{1}, k}\left(y_{1}, v\right)$. By definition,

$$
\sum_{y_{1}, y_{2}} F_{m_{1}, m_{2}}^{(i)}\left(y_{1}, y_{2}\right) \leqslant \sum_{y_{1}, y_{2}, v, z} \sum_{k=m_{1}}^{m_{2}} \sum_{j=0}^{k} F_{m_{1}, k}\left(y_{1}, v ; 1_{(z, j)}\right) \tau_{m_{2}-j}\left(y_{2}-z\right) \tau_{m_{2}-k}^{(i)}\left(y_{2}-v\right) .
$$

It follows from (7.24) that $\sum_{x} \tau_{m}^{(i)}(x) \leqslant p K \leqslant C K$, and that

$$
\sum_{y_{2}} \tau_{m_{2}-j}\left(y_{2}-z\right) \tau_{m_{2}-k}^{(i)}\left(y_{2}-v\right) \leqslant C \beta^{i}\left(m_{2}-j+1\right)^{-d / 2},
$$

since $j<m_{2}$ for $i=1$. This leads to

$$
\sum_{y_{1}, y_{2}} F_{m_{1}, m_{2}}^{(i)}(u, x) \leqslant \sum_{y_{1}, v, z} \sum_{k=m_{1}}^{m_{2}} \sum_{j=0}^{k} F_{m_{1}, k}\left(y_{1}, v ; 1_{(z, j)}\right) C \beta^{i}\left(m_{2}-j+1\right)^{-d / 2} .
$$

By Lemma 7.8(a),

$$
\sum_{y_{1}, v, z} F_{m_{1}, k}\left(y_{1}, v ; 1_{(z, j)}\right) \leqslant C C_{F} b_{m_{1}, k}
$$

holds uniformly in $0 \leqslant j \leqslant k$. Therefore,

$$
\begin{aligned}
\sum_{y_{1}, y_{2}} F_{m_{1}, m_{2}}^{(i)}\left(y_{1}, y_{2}\right) & \leqslant C C_{F} \beta^{i} \sum_{k=m_{1}}^{m_{2}} b_{m_{1}, k} \sum_{j=0}^{k}\left(m_{2}-j+1\right)^{-d / 2} \\
& \leqslant C C_{F} \beta^{i} \sum_{k=m_{1}}^{m_{2}} b_{m_{1}, k}\left(m_{2}-k+1\right)^{-(d-2) / 2} \\
& =C C_{F} \beta^{i}\left(m_{1}+1\right)^{-(d-2) / 2} \sum_{k=m_{1}}^{m_{2}}\left(k-m_{1}+1\right)^{-(d-2) / 2}\left(m_{2}-k+1\right)^{-(d-2) / 2} \\
& \leqslant C C_{F} \beta^{i} b_{m_{1}, m_{2}},
\end{aligned}
$$

where we used [7, Lemma 2.6(i)] and $d>4$ in the last step.

The statement concerning Construction $E$ then follows from the comment under Definition 7.3.

Lemma 7.10. Let $d>2$ and assume that $m_{2}>m_{1}$. Suppose that the diagram $F_{m}(y)$ satisfies

$$
\sum_{y} F_{m}(y) \leqslant C_{F}(m+1)^{-d / 2}
$$

with this bound obtained by associating $l^{1}$ - and $l^{\infty}$-norms to diagram lines and by using (7.24) to estimate these norms. For $m_{2}>m_{1}$, let

$$
\widetilde{F}_{m_{1}, m_{2}}\left(y_{1}, y_{2}\right)=\sum_{k, j=0}^{m_{1}} \sum_{z, w} F_{m_{1}}\left(y_{1} ; 1(z, j), 1(w, k)\right) \tau_{m_{2}-k}\left(y_{2}-w\right) \tau_{m_{2}-j}\left(y_{2}-z\right) .
$$

Then

$$
\sum_{y_{1}, y_{2}} \widetilde{F}_{m_{1}, m_{2}}\left(y_{1}, y_{2}\right) \leqslant C C_{F} \beta b_{m_{1}, m_{2}},
$$

with $C$ independent of $F$ and $C_{F}$, and with the bound obtained by associating $l^{1}$ - and $l^{\infty}$-norms to diagram lines and by using (7.24) to estimate these norms. 
Proof. By the symmetry between $k$ and $j$,

$$
\widetilde{F}_{m_{1}, m_{2}}\left(y_{1}, y_{2}\right) \leqslant 2 \sum_{z, w} \sum_{k=0}^{m_{1}} \sum_{j=k}^{m_{1}} \tau_{m_{2}-k}\left(y_{2}-z\right) \tau_{m_{2}-j}\left(y_{2}-w\right) F_{m_{1}}\left(y_{1} ; 1(z, j), 1(w, k)\right) .
$$

Therefore, by (7.24) and the fact that $m_{2}>m_{1} \geqslant j$,

$$
\sum_{y_{1}, y_{2}} \widetilde{F}_{m_{1}, m_{2}}\left(y_{1}, y_{2}\right) \leqslant 2 \sum_{k=0}^{m_{1}} \sum_{j=k}^{m_{1}} K^{2} \beta\left(m_{2}-k+1\right)^{-d / 2} \sum_{y_{1}} F_{m_{1}}\left(y_{1} ; 1(k), 1(j)\right) .
$$

It follows from Lemma 7.8(a) that

$$
\sum_{y_{1}} F_{m_{1}}\left(y_{1} ; 1(k), 1(j)\right) \leqslant C C_{F}\left(m_{1}+1\right)^{-d / 2},
$$

uniformly in $k, j$. Since $m_{2}>m_{1}$, we conclude that

$$
\begin{aligned}
\sum_{y_{1}, y_{2}} \widetilde{F}_{m_{1}, m_{2}}\left(y_{1}, y_{2}\right) & \leqslant C C_{F} \beta\left(m_{1}+1\right)^{-d / 2} \sum_{k=0}^{m_{1}} \sum_{j=k}^{m_{1}}\left(m_{2}-k+1\right)^{-d / 2} \\
& \leqslant C C_{F} \beta\left(m_{1}+1\right)^{-(d-2) / 2} \sum_{k=0}^{m_{1}}\left(m_{2}-k+1\right)^{-d / 2} \\
& \leqslant C C_{F} \beta b_{m_{1}, m_{2}} . \quad \square
\end{aligned}
$$

\subsubsection{Proof of Proposition 7.7}

Now we prove Proposition 7.7. According to the definitions below (7.18), it suffices to prove that, for $N \geqslant 0$,

$$
\sum_{y_{1}, y_{2}} R_{m_{1}, m_{2}}^{(N)}\left(y_{1}, y_{2}\right) \leqslant C(C \beta)^{N} b_{m_{1}, m_{2}},
$$

and that for $N \geqslant 1$,

$$
\sum_{y_{1}, y_{2}} Q_{m_{1}, m_{2}}^{(N)}\left(y_{1}, y_{2}\right) \leqslant(C \beta)^{N+1} b_{m_{1}, m_{2}},
$$

since (7.21), (7.22) then follow from Lemma 7.9.

We start with the proof of (7.40). Let

$$
S_{m_{1}, m_{2}}^{(N)}\left(y_{1}, y_{2}\right)=P_{m_{1}}^{(N)}\left(y_{1} ; \ell\left(y_{2}, m_{1}\right)\right) \delta_{m_{1}, m_{2}} .
$$

By (7.17), $R_{m_{1}, m_{2}}^{(N)}\left(y_{1}, y_{2}\right)$ is obtained from $S_{m_{1}, k}^{(N)}\left(y_{1}, v\right)$ by applying Construction $2_{(v, k)}^{(0)}\left(y_{2}, m_{2}\right)$. Therefore, by Lemma 7.9, to prove (7.40) it suffices to show that

$$
\sum_{y_{1}, y_{2}} S_{m_{1}, m_{2}}^{(N)}\left(y_{1}, y_{2}\right) \leqslant C(C \beta)^{N} b_{m_{1}, m_{2}} .
$$

Lemma 7.8(b), together with (6.2) and the fact that $P^{(N)}(y)$ has $\mathcal{L} \leqslant 4$ (as noted above Lemma 7.8), gives the desired result that

$$
\begin{aligned}
\sum_{y_{1}, y_{2}} S_{m_{1}, m_{2}}^{(N)}\left(y_{1}, y_{2}\right) & =\sum_{y_{1}, y_{2}} P_{m_{1}}^{(N)}\left(y_{1} ; \ell\left(y_{2}, m_{1}\right)\right) \delta_{m_{1}, m_{2}} \\
& \leqslant C(C \beta)^{N}\left(m_{1}+1\right)^{-(d-2) / 2} \delta_{m_{1}, m_{2}}=C(C \beta)^{N} b_{m_{1}, m_{2}} \delta_{m_{1}, m_{2}} .
\end{aligned}
$$

Next, we prove (7.41). In (7.18), a factor $\tau(\boldsymbol{a}-\boldsymbol{w}) P^{(0)}\left(\boldsymbol{z}, \boldsymbol{y}_{2} ; \boldsymbol{a}\right)$ arises, where $\boldsymbol{w}$ is the location where Construction $\ell(\boldsymbol{a})$ is applied. We write $\boldsymbol{a}=(a, m)$ and $\boldsymbol{y}_{1}=\left(y_{1}, m_{1}\right)$, and note that (7.18) requires that $m>m_{1}$. We regard $\tau(\boldsymbol{a}-\boldsymbol{w}) P^{(0)}\left(\boldsymbol{z}, \boldsymbol{y}_{2} ; \boldsymbol{a}\right)$ as the result of Construction $2_{\boldsymbol{a}}^{(0)}\left(\boldsymbol{y}_{2}\right)$ applied to $\tau(\boldsymbol{a}-\boldsymbol{w}) \tau(\boldsymbol{a}-\boldsymbol{z})$, where the unique admissible line is $\tau(\boldsymbol{a}-\boldsymbol{z})$. Let

$$
\tilde{S}_{m_{1}, m}^{(N)}\left(y_{1}, a\right)=\sum_{k, j=0}^{m_{1}} P_{m_{1}}^{(N)}\left(y_{1} ; 1(z, j), 1(w, k)\right) \tau_{m-k}(a-w) \tau_{m-j}(a-z) .
$$


Then $Q_{m_{1}, m_{2}}^{(N)}\left(y_{1}, y_{2}\right)$ arises from an application of Construction $2_{\boldsymbol{a}}^{(0)}\left(\boldsymbol{y}_{2}\right)$ to the second argument of $\tilde{S}_{m_{1}, m}^{(N)}\left(y_{1}, a\right)$. By Lemma 7.9, it suffices to prove that if $m>m_{1}$, then

$$
\sum_{y_{1}, a} \tilde{S}_{m_{1}, m}^{(N)}\left(y_{1}, a\right) \leqslant(C \beta)^{N+1} b_{m_{1}, m} .
$$

The bound (7.46) follows from Lemma 7.10 and (6.2), and this completes the proof of (7.41).

\subsection{Proof of Proposition 7.6}

In this section, we prove Proposition 7.6. We begin in Section 7.4.1 with some key estimates for the proof. In Section 7.4.2, we prove (7.19), (7.20) for the case $K=0$, and we extend these inequalities to $K \geqslant 1$ in Section 7.4.3. In Section 7.4.4, we prove the final statement of Proposition 7.6.

\subsubsection{Key estimates for proof of Proposition 7.6}

For $\boldsymbol{v}, \boldsymbol{x}$ and $m \geqslant 0$ such that $m_{\boldsymbol{v}} \leqslant m \leqslant m_{\boldsymbol{x}}$, let

$$
\begin{aligned}
& V_{m}(\boldsymbol{v}, \boldsymbol{x})=\bigcup_{z: m_{z} \leqslant m}\{\boldsymbol{v} \rightarrow z \Longrightarrow \boldsymbol{x}\}, \\
& \mathcal{E}_{m}(\boldsymbol{v}, \boldsymbol{x} ; A)=\bigcup_{\boldsymbol{a} \in A} \bigcup_{z: m_{z} \geqslant m}(\boldsymbol{v} \rightarrow \boldsymbol{z}) \circ(z \rightarrow \boldsymbol{x}) \circ(\boldsymbol{z} \rightarrow \boldsymbol{a}) \circ(\boldsymbol{a} \rightarrow \boldsymbol{x}) .
\end{aligned}
$$

The events $V_{m}(\boldsymbol{v}, \boldsymbol{x})$ and $\mathcal{E}_{m}(\boldsymbol{v}, \boldsymbol{x} ; A)$ are depicted in Fig. 9. By (6.12),

$$
\mathcal{E}(\boldsymbol{v}, \boldsymbol{x} ; A)=\mathcal{E}_{m_{\boldsymbol{v}}}(\boldsymbol{v}, \boldsymbol{x} ; A) .
$$

Also, for later use, we note that

$$
V_{m}(\boldsymbol{v}, \boldsymbol{x}) \subseteq \bigcup_{z: m_{z} \leqslant m} \bigcup_{t: m_{t}=m}(\boldsymbol{v} \rightarrow \boldsymbol{z}) \cap((\boldsymbol{v} \rightarrow \boldsymbol{t}) \circ(\boldsymbol{t} \rightarrow \boldsymbol{x}) \circ(z \rightarrow \boldsymbol{x})) .
$$

To prove (7.50), we observe that if $m_{z} \leqslant m \leqslant m_{x}$, then

$$
(z \Longrightarrow x)=\bigcup_{t: m_{t}=m}(z \rightarrow t) \circ(t \rightarrow x) \circ(z \rightarrow x),
$$

and hence, by (7.47),

$$
\begin{aligned}
V_{m}(\boldsymbol{v}, \boldsymbol{x}) & =\bigcup_{z: m_{z} \leqslant m} \bigcup_{t: m_{t}=m}(\boldsymbol{v} \rightarrow \boldsymbol{z}) \cap((\boldsymbol{z} \rightarrow \boldsymbol{t}) \circ(\boldsymbol{t} \rightarrow \boldsymbol{x}) \circ(\boldsymbol{z} \rightarrow \boldsymbol{x})) \\
& \subseteq \bigcup_{z: m_{z} \leqslant m} \bigcup_{t: m_{t}=m}(\boldsymbol{v} \rightarrow \boldsymbol{z}) \cap((\boldsymbol{v} \rightarrow \boldsymbol{t}) \circ(\boldsymbol{t} \rightarrow \boldsymbol{x}) \circ(\boldsymbol{z} \rightarrow \boldsymbol{x})) .
\end{aligned}
$$

The following proposition provides the key estimates for the proof of Proposition 7.6.

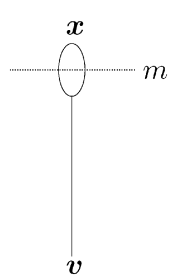

(a)

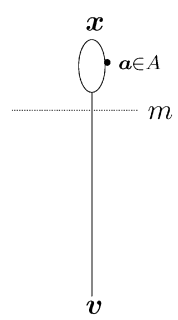

(b)

Fig. 9. Schematic representations of the events (a) $V_{m}(\boldsymbol{v}, \boldsymbol{x})$ and (b) $\mathcal{E}_{m}(\boldsymbol{v}, \boldsymbol{x} ; A)$. 
Proposition 7.11. Let $p$ and $d$ be arbitrary and assume the weak assumption on D. For $N, M \geqslant 0, m_{1} \geqslant 1$, and $\boldsymbol{a}, \boldsymbol{y}_{2} \in \Lambda$,

$$
\begin{aligned}
& \sum_{\left(\boldsymbol{u}_{N}, \boldsymbol{v}_{N}\right)} J_{\boldsymbol{u}_{N}, \boldsymbol{v}_{N}} M_{\boldsymbol{u}_{N}}^{(N+1)}\left(I\left[V_{m_{1}-1}\left(\boldsymbol{v}_{N-1}, \boldsymbol{y}_{2}\right)\right] \Pi_{m_{1}}^{(M)}\left(\boldsymbol{v}_{N} ; \tilde{C}_{N}\right)\right) \leqslant p \sum_{y_{1}} R^{(N+M)}\left(\left(y_{1}, m_{1}-1\right), \boldsymbol{y}_{2}\right), \\
& \sum_{\left(\boldsymbol{u}_{N}, \boldsymbol{v}_{N}\right)} J_{\boldsymbol{u}_{N}, \boldsymbol{v}_{N}} M_{\boldsymbol{u}_{N}}^{(N+1)}\left(I\left[V_{m_{1}-1}\left(\boldsymbol{v}_{N-1}, \boldsymbol{y}_{2}\right)\right] I\left[\boldsymbol{a} \in \tilde{C}_{N}\right] \Pi_{m_{1}}^{(M)}\left(\boldsymbol{v}_{N} ; \tilde{C}_{N}\right)\right) \\
& \quad \leqslant p \sum_{y_{1}} R^{(N+M)}\left(\left(y_{1}, m_{1}-1\right), \boldsymbol{y}_{2} ; \ell(\boldsymbol{a})\right), \\
& \sum_{\left(\boldsymbol{u}_{N}, \boldsymbol{v}_{N}\right)} J_{\boldsymbol{u}_{N}, \boldsymbol{v}_{N}} M_{\boldsymbol{u}_{N}}^{(N+1)}\left(I\left[\mathcal{E}_{m_{1}}\left(\boldsymbol{v}_{N-1}, \boldsymbol{y}_{2} ; \tilde{C}_{N-1}\right)\right] \Pi_{m_{1}}^{(M)}\left(\boldsymbol{v}_{N} ; \tilde{C}_{N}\right)\right) \leqslant p \sum_{y_{1}} Q^{(N+M)}\left(\left(y_{1}, m_{1}-1\right), \boldsymbol{y}_{2}\right), \\
& \sum_{\left(\boldsymbol{u}_{N}, \boldsymbol{v}_{N}\right)} J_{\boldsymbol{u}_{N}, \boldsymbol{v}_{N}} M_{\boldsymbol{u}_{N}}^{(N+1)}\left(I\left[\mathcal{E}_{m_{1}}\left(\boldsymbol{v}_{N-1}, \boldsymbol{y}_{2} ; \tilde{C}_{N-1}\right)\right] I\left[\boldsymbol{a} \in \tilde{C}_{N}\right] \Pi_{m_{1}}^{(M)}\left(\boldsymbol{v}_{N} ; \tilde{C}_{N}\right)\right) \\
& \quad \leqslant p \sum_{y_{1}} Q^{(N+M)}\left(\left(y_{1}, m_{1}-1\right), \boldsymbol{y}_{2} ; \ell(\boldsymbol{a})\right) .
\end{aligned}
$$

The proof of Proposition 7.11 relies on three lemmas which we state and prove before proving the proposition. Lemmas 7.12-7.13 are used to prove (7.53), (7.54), and Lemma 7.14 is used to prove (7.55), (7.56).

Lemma 7.12. Let $p$ and $d$ be arbitrary and assume the weak assumption on $D$. For $N \geqslant 0, m_{1} \geqslant 1, \boldsymbol{v}_{N-1}, \boldsymbol{u}_{N}, \boldsymbol{y}_{2} \in \Lambda$, and $\overrightarrow{\boldsymbol{x}}$ with 0,1 or 2 components in $\Lambda$,

$$
\begin{aligned}
& \mathbb{P}_{N}\left(E^{\prime}\left(\boldsymbol{v}_{N-1}, \boldsymbol{u}_{N} ; \tilde{C}_{N-1}\right) \cap V_{m_{1}-1}\left(\boldsymbol{v}_{N-1}, \boldsymbol{y}_{2}\right) \cap\left\{\overrightarrow{\boldsymbol{x}} \in \tilde{C}_{N}\right\}\right) \\
& \leqslant \sum_{\lambda} P^{(0)}\left(\boldsymbol{v}_{N-1}, \boldsymbol{u}_{N} ; \tilde{C}_{N-1}, \ell^{\lambda}(\overrightarrow{\boldsymbol{x}}), V_{m_{1}-1}\left(\boldsymbol{y}_{2}\right)\right),
\end{aligned}
$$

where Construction $\ell^{\lambda}(\overrightarrow{\boldsymbol{x}})$ is obtained by performing Construction $\ell^{\lambda}\left(\boldsymbol{x}_{1}\right)$ followed by Constructions $\ell\left(\boldsymbol{x}_{i}\right)$ for all other components $\boldsymbol{x}_{i}$ with $i>1$, and the sum over $\lambda$ follows the convention of Remark 6.2.

Proof. By (6.12) and (6.3), (6.4), the event $E^{\prime}\left(\boldsymbol{v}_{N-1}, \boldsymbol{u}_{N} ; \tilde{C}_{N-1}\right)$ implies that the disjoint connections necessary to obtain an upper bound $P^{(0)}\left(\boldsymbol{v}_{N-1}, \boldsymbol{u}_{N} ; \tilde{C}_{N-1}\right)$ are present. The additional connections implied by $\left\{\overrightarrow{\boldsymbol{x}} \in \tilde{C}_{N}\right\}$ can be accounted for in an upper bound by an application of Construction $\ell^{\lambda}(\vec{x})$ to $P^{(0)}\left(\boldsymbol{v}_{N-1}, \boldsymbol{u}_{N} ; \tilde{C}_{N-1}\right)$. To understand the effect of the event $V_{m_{1}-1}\left(\boldsymbol{v}_{N-1}, \boldsymbol{y}_{2}\right)$, we use (7.50). The connection $\boldsymbol{v}_{N-1} \rightarrow \boldsymbol{t}=\left(t, m_{1}-1\right)$ in (7.50) can be accounted for by an application of Construction $\ell(t)$, and the remaining connections in (7.50) can then be accounted for by an application of Construction $2_{\boldsymbol{t}}^{(0)}\left(\boldsymbol{y}_{2}\right)$. By the equivalent definition of Construction $V_{m_{1}-1}\left(\boldsymbol{y}_{2}\right)$ formulated below Definition 7.5, this completes the proof.

Lemma 7.13. Under the same conditions as in Lemma 7.12,

$$
M_{\boldsymbol{u}_{N}}^{(N+1)}\left(I\left[V_{m_{1}-1}\left(\boldsymbol{v}_{N-1}, \boldsymbol{y}_{2}\right)\right] I\left[\overrightarrow{\boldsymbol{x}} \in \tilde{C}_{N}\right]\right) \leqslant \sum_{\lambda} P^{(N)}\left(\boldsymbol{u}_{N} ; \ell^{\lambda}(\overrightarrow{\boldsymbol{x}}), V_{m_{1}-1}\left(\boldsymbol{y}_{2}\right)\right) .
$$

Proof. For $N=0$, we must bound $M_{\boldsymbol{u}_{0}}^{(1)}\left(I\left[V_{m_{1}-1}\left(\mathbf{0}, \boldsymbol{y}_{2}\right)\right] I\left[\overrightarrow{\boldsymbol{x}} \in \tilde{C}_{0}\right]\right)$. For $N \geqslant 1$, (3.35) gives

$$
\begin{aligned}
& M_{\boldsymbol{u}_{N}}^{(N+1)}\left(I\left[V_{m_{1}-1}\left(\boldsymbol{v}_{N-1}, \boldsymbol{y}_{2}\right)\right] I\left[\overrightarrow{\boldsymbol{x}} \in \tilde{C}_{N}\right]\right) \\
& \quad=\sum_{\left(\boldsymbol{u}_{N-1}, \boldsymbol{v}_{N-1}\right)} J_{\boldsymbol{u}_{N-1}, \boldsymbol{v}_{N-1}} M_{\boldsymbol{u}_{N-1}}^{(N)}\left(M_{\boldsymbol{v}_{N-1}, \boldsymbol{u}_{N} ; \tilde{C}_{N-1}}^{(1)}\left(I\left[V_{m_{1}-1}\left(\boldsymbol{v}_{N-1}, \boldsymbol{y}_{2}\right)\right] I\left[\overrightarrow{\boldsymbol{x}} \in \tilde{C}_{N}\right]\right)\right) .
\end{aligned}
$$

The statement of Lemma 7.12 can be rewritten as

$$
M_{\boldsymbol{v}_{N-1}, \boldsymbol{u}_{N}}^{(1)} \tilde{C}_{N-1}\left(I\left[V_{m_{1}-1}\left(\boldsymbol{v}_{N-1}, \boldsymbol{y}_{2}\right)\right] I\left[\overrightarrow{\boldsymbol{x}} \in \tilde{C}_{N}\right]\right) \leqslant \sum_{\lambda} P^{(0)}\left(\boldsymbol{v}_{N-1}, \boldsymbol{u}_{N} ; \tilde{C}_{N-1}, \ell^{\lambda}(\overrightarrow{\boldsymbol{x}}), V_{m_{1}-1}\left(\boldsymbol{y}_{2}\right)\right) .
$$


With (4.15) and (6.4), this proves (7.58) for $N=0$. For $N \geqslant 1$, we substitute (7.60) into (7.59) to obtain

$$
\begin{aligned}
& M_{\boldsymbol{u}_{N}}^{(N+1)}\left(I\left[V_{m_{1}-1}\left(\boldsymbol{v}_{N-1}, \boldsymbol{y}_{2}\right)\right] I\left[\overrightarrow{\boldsymbol{x}} \in \tilde{C}_{N}\right]\right) \\
& \quad \leqslant \sum_{\lambda} \sum_{\boldsymbol{w}^{\prime}} \sum_{\left(\boldsymbol{u}_{N-1}, \boldsymbol{v}_{N-1}\right)} J_{\boldsymbol{u}_{N-1}, \boldsymbol{v}_{N-1}} M_{\boldsymbol{u}_{N-1}}^{(N)}\left(I\left[\boldsymbol{w}^{\prime} \in \tilde{C}_{N-1}\right]\right) P^{(0)}\left(\boldsymbol{v}_{N-1}, \boldsymbol{u}_{N} ; \boldsymbol{w}^{\prime}, \ell^{\lambda}(\overrightarrow{\boldsymbol{x}}), V_{m_{1}-1}\left(\boldsymbol{y}_{2}\right)\right) .
\end{aligned}
$$

Application of (6.11) then gives

$$
\begin{aligned}
& M_{\boldsymbol{u}_{N}}^{(N+1)}\left(I\left[V_{m_{1}-1}\left(\boldsymbol{v}_{N-1}, \boldsymbol{y}_{2}\right)\right] I\left[\overrightarrow{\boldsymbol{x}} \in \tilde{C}_{N}\right]\right) \\
& \quad \leqslant \sum_{\lambda, \lambda^{\prime}} \sum_{\boldsymbol{w}^{\prime}} \sum_{\left(\boldsymbol{u}_{N-1}, \boldsymbol{v}_{N-1}\right)} J_{\boldsymbol{u}_{N-1}, \boldsymbol{v}_{N-1}} P^{(N-1)}\left(\boldsymbol{u}_{N-1} ; \ell^{\lambda^{\prime}}\left(\boldsymbol{w}^{\prime}\right)\right) P^{(0)}\left(\boldsymbol{v}_{N-1}, \boldsymbol{u}_{N} ; \boldsymbol{w}^{\prime}, \ell^{\lambda}(\overrightarrow{\boldsymbol{x}}), V_{m_{1}-1}\left(\boldsymbol{y}_{2}\right)\right) \\
& \quad \leqslant \sum_{\lambda} P^{(N)}\left(\boldsymbol{u}_{N} ; \ell^{\lambda}(\overrightarrow{\boldsymbol{x}}), V_{m_{1}-1}\left(\boldsymbol{y}_{2}\right)\right),
\end{aligned}
$$

where we have used a slight extension of (6.21) in the last inequality. This proves (7.58).

Lemma 7.14. Under the same conditions as in Lemma 7.12, and with the additional assumption that $m_{\boldsymbol{x}_{1}}, m_{\boldsymbol{u}_{N}} \leqslant m_{1}$

$$
M_{\boldsymbol{u}_{N}}^{(N+1)}\left(I\left[\mathcal{E}_{m_{1}}\left(\boldsymbol{v}_{N-1}, \boldsymbol{y}_{2} ; \tilde{C}_{N-1}\right)\right] I\left[\overrightarrow{\boldsymbol{x}} \in \tilde{C}_{N}\right]\right) \leqslant \sum_{\lambda} P^{(N)}\left(\boldsymbol{u}_{N} ; \ell^{\lambda}\left(\boldsymbol{x}_{1}\right), \mathcal{E}_{m_{1}}\left(\boldsymbol{y}_{2}\right), \ell\left(\boldsymbol{x}_{2}\right)\right) .
$$

Proof. For $N=0$ and $m_{1} \geqslant 1$, the event $\mathcal{E}_{m_{1}}\left(\boldsymbol{v}_{N-1}, \boldsymbol{y}_{2} ; \tilde{C}_{N-1}\right)=\mathcal{E}_{m_{1}}\left(\mathbf{0}, \boldsymbol{y}_{2} ;\{\mathbf{0}\}\right)$ is empty, so we may assume that $N \geqslant 1$. For $N=1$, we must bound $M_{\boldsymbol{u}_{1}}^{(2)}\left(I\left[\mathcal{E}_{m_{1}}\left(\boldsymbol{v}_{1}, \boldsymbol{y}_{2} ; \tilde{C}_{0}\right)\right] I\left[\overrightarrow{\boldsymbol{x}} \in \tilde{C}_{1}\right]\right)$. For $N \geqslant 2$, it follows from (3.38) that

$$
\begin{aligned}
& M_{\boldsymbol{u}_{N}}^{(N+1)}\left(I\left[\mathcal{E}_{m_{1}}\left(\boldsymbol{v}_{N-1}, \boldsymbol{y}_{2} ; \tilde{C}_{N-1}\right)\right] I\left[\overrightarrow{\boldsymbol{x}} \in \tilde{C}_{N}\right]\right) \\
& \quad=\sum_{\left(\boldsymbol{u}_{N-2}, \boldsymbol{v}_{N-2}\right)} J_{\boldsymbol{u}_{N-2}, \boldsymbol{v}_{N-2}} M_{\boldsymbol{u}_{N-2}}^{(N-1)}\left(M_{\boldsymbol{v}_{N-2}, \boldsymbol{u}_{N} ; \tilde{C}_{N-2}}^{(2)}\left(I\left[\mathcal{E}_{m_{1}}\left(\boldsymbol{v}_{N-1}, \boldsymbol{y}_{2} ; \tilde{C}_{N-1}\right)\right] I\left[\overrightarrow{\boldsymbol{x}} \in \tilde{C}_{N}\right]\right)\right) .
\end{aligned}
$$

We will show below that if $m_{\boldsymbol{x}_{1}}, m_{\boldsymbol{u}_{N}} \leqslant m_{1}$, then

$$
\begin{aligned}
& M_{\boldsymbol{v}_{N-2}, \boldsymbol{u}_{N} ; \tilde{C}_{N-2}}^{(2)}\left(I\left[\mathcal{E}_{m_{1}}\left(\boldsymbol{v}_{N-1}, \boldsymbol{y}_{2} ; \tilde{C}_{N-1}\right)\right] I\left[\overrightarrow{\boldsymbol{x}} \in \tilde{C}_{N}\right]\right) \\
& \quad \leqslant \sum_{\lambda} P^{(1)}\left(\boldsymbol{v}_{N-2}, \boldsymbol{u}_{N} ; \tilde{C}_{N-2}, \ell^{\lambda}\left(\boldsymbol{x}_{1}\right), \mathcal{E}_{m_{1}}\left(\boldsymbol{y}_{2}\right), \ell\left(\boldsymbol{x}_{2}\right)\right) .
\end{aligned}
$$

Then (7.63) follows from (7.65), as in (7.61), (7.62).

It remains to prove (7.65). For simplicity, we consider in detail the case $\overrightarrow{\boldsymbol{x}}=\boldsymbol{x}_{1}$. By (3.35),

$$
\begin{gathered}
M_{\boldsymbol{v}_{N-2}, \boldsymbol{u}_{N} ; \tilde{C}_{N-2}}^{(2)}\left(I\left[\mathcal{E}_{m_{1}}\left(\boldsymbol{v}_{N-1}, \boldsymbol{y}_{2} ; \tilde{C}_{N-1}\right)\right] I\left[\boldsymbol{x}_{1} \in \tilde{C}_{N}\right]\right) \\
=\sum_{\left(\boldsymbol{u}_{N-1}, \boldsymbol{v}_{N-1}\right)} J_{\boldsymbol{u}_{N-1}, \boldsymbol{v}_{N-1}} M_{\boldsymbol{v}_{N-2}, \boldsymbol{u}_{N-1} ; \tilde{C}_{N-2}}^{(1)}\left(\mathbb { P } _ { N } \left(E^{\prime}\left(\boldsymbol{v}_{N-1}, \boldsymbol{u}_{N} ; \tilde{C}_{N-1}\right)\right.\right. \\
\left.\left.\quad \cap \mathcal{E}_{m_{1}}\left(\boldsymbol{v}_{N-1}, \boldsymbol{y}_{2} ; \tilde{C}_{N-1}\right) \cap\left\{\boldsymbol{x}_{1} \in \tilde{C}_{N}\right\}\right)\right) .
\end{gathered}
$$

By (7.48),

$$
\begin{aligned}
& \mathbb{P}_{N}\left(E^{\prime}\left(\boldsymbol{v}_{N-1}, \boldsymbol{u}_{N} ; \tilde{C}_{N-1}\right) \cap \mathcal{E}_{m_{1}}\left(\boldsymbol{v}_{N-1}, \boldsymbol{y}_{2} ; \tilde{C}_{N-1}\right) \cap\left\{\boldsymbol{x}_{1} \in \tilde{C}_{N}\right\}\right) \\
& \quad \leqslant \sum_{z: m_{z} \geqslant m_{1}} \sum_{\boldsymbol{w} \in \tilde{C}_{N-1}} \mathbb{P}_{N}\left(E^{\prime}\left(\boldsymbol{v}_{N-1}, \boldsymbol{u}_{N} ; \tilde{C}_{N-1}\right) \cap\left\{\boldsymbol{z}, \boldsymbol{x}_{1} \in \tilde{C}_{N}\right\} \cap\left\{(z \rightarrow \boldsymbol{w}) \circ\left(z \rightarrow \boldsymbol{y}_{2}\right) \circ\left(\boldsymbol{w} \rightarrow \boldsymbol{y}_{2}\right)\right\}\right) .
\end{aligned}
$$

Using (6.14), the Markov property, the fact that $m_{\boldsymbol{x}_{1}}, m_{\boldsymbol{u}_{N}} \leqslant m_{z}$, and the BK inequality, we obtain

$$
\begin{aligned}
& \mathbb{P}_{N}\left(E^{\prime}\left(\boldsymbol{v}_{N-1}, \boldsymbol{u}_{N} ; \tilde{C}_{N-1}\right) \cap\left\{\boldsymbol{z}, \boldsymbol{x}_{1} \in \tilde{C}_{N}\right\} \cap\left\{(z \rightarrow \boldsymbol{w}) \circ\left(\boldsymbol{z} \rightarrow \boldsymbol{y}_{2}\right) \circ\left(\boldsymbol{w} \rightarrow \boldsymbol{y}_{2}\right)\right\}\right) \\
& \quad=\mathbb{P}_{N}\left(E^{\prime}\left(\boldsymbol{v}_{N-1}, \boldsymbol{u}_{N} ; \tilde{C}_{N-1}\right) \cap\left\{\boldsymbol{z}, \boldsymbol{x}_{1} \in \tilde{C}_{N}\right\}\right) \mathbb{P}_{N}\left((\boldsymbol{z} \rightarrow \boldsymbol{w}) \circ\left(\boldsymbol{z} \rightarrow \boldsymbol{y}_{2}\right) \circ\left(\boldsymbol{w} \rightarrow \boldsymbol{y}_{2}\right)\right) \\
& \quad \leqslant \sum_{\lambda} P^{(0)}\left(\boldsymbol{v}_{N-1}, \boldsymbol{u}_{N} ; \tilde{C}_{N-1}, \ell^{\lambda}\left(\boldsymbol{x}_{1}\right), \ell(\boldsymbol{z})\right) \tau(\boldsymbol{w}-\boldsymbol{z}) \tau\left(\boldsymbol{y}_{2}-\boldsymbol{z}\right) \tau\left(\boldsymbol{y}_{2}-\boldsymbol{w}\right) .
\end{aligned}
$$


Also, it is a straightforward extension of (6.14) that

$$
M_{\boldsymbol{v}, \boldsymbol{y} ; A}^{(1)}\left(I\left[\boldsymbol{v} \rightarrow \boldsymbol{w}, \boldsymbol{v} \rightarrow \boldsymbol{w}^{\prime}\right]\right) \leqslant \sum_{\lambda^{\prime}} P^{(0)}\left(\boldsymbol{v}, \boldsymbol{y} ; A, \ell^{\lambda^{\prime}}(\boldsymbol{w}), \ell\left(\boldsymbol{w}^{\prime}\right)\right) .
$$

We substitute (7.68) into (7.67) and substitute the result into (7.66). This gives

$$
\begin{aligned}
& M_{\boldsymbol{v}_{N-2}, \boldsymbol{u}_{N}}^{(2)}, \tilde{C}_{N-2} \\
& \quad \leqslant \sum_{\left(\boldsymbol{u}_{N-1}, \boldsymbol{v}_{N-1}\right)} J_{\boldsymbol{u}_{N-1}, \boldsymbol{v}_{N-1}} \sum_{\boldsymbol{w}, \boldsymbol{w}^{\prime}, z: m_{z} \geqslant m_{1}} M_{\boldsymbol{v}_{N-2}, \boldsymbol{u}_{N-1} ; \tilde{C}_{N-2}}^{(1)}\left(I\left[\boldsymbol{w}, \boldsymbol{w}^{\prime} \in \tilde{C}_{N-1}\right]\right) \\
& \quad \times \sum_{\lambda} P^{(0)}\left(\boldsymbol{v}_{N-1}, \boldsymbol{u}_{N} ; \boldsymbol{w}^{\prime}, \ell^{\lambda}\left(\boldsymbol{x}_{1}\right), \ell(\boldsymbol{z})\right) \tau(\boldsymbol{w}-\boldsymbol{z}) \tau\left(\boldsymbol{y}_{2}-\boldsymbol{z}\right) \tau\left(\boldsymbol{y}_{2}-\boldsymbol{w}\right) \\
& \quad \leqslant \sum_{\lambda, \lambda^{\prime}\left(\boldsymbol{u}_{N-1}, \boldsymbol{v}_{N-1}\right)} J_{\boldsymbol{u}_{N-1}, \boldsymbol{v}_{N-1}} \sum_{\boldsymbol{w}, \boldsymbol{w}^{\prime}, z: m_{z} \geqslant m_{1}} P^{(0)}\left(\boldsymbol{v}_{N-2}, \boldsymbol{u}_{N-1} ; \tilde{C}_{N-2}, \ell^{\lambda^{\prime}}\left(\boldsymbol{w}^{\prime}\right), \ell(\boldsymbol{w})\right) \\
& \quad \times P^{(0)}\left(\boldsymbol{v}_{N-1}, \boldsymbol{u}_{N} ; \boldsymbol{w}^{\prime}, \ell^{\lambda}\left(\boldsymbol{x}_{1}\right), \ell(\boldsymbol{z})\right) \tau(\boldsymbol{w}-\boldsymbol{z}) \tau\left(\boldsymbol{y}_{2}-\boldsymbol{z}\right) \tau\left(\boldsymbol{y}_{2}-\boldsymbol{w}\right),
\end{aligned}
$$

where we have used (7.69) in the second inequality. It can then be concluded from (7.13), (6.21) and (6.3), by drawing the picture, that

$$
M_{\boldsymbol{v}_{N-2}, \boldsymbol{u}_{N} ; \tilde{C}_{N-2}}^{(2)}\left(I\left[\mathcal{E}_{m_{1}}\left(\boldsymbol{v}_{N-1}, \boldsymbol{y}_{2} ; \tilde{C}_{N-1}\right)\right] I\left[\boldsymbol{x}_{1} \in \tilde{C}_{N}\right]\right) \leqslant \sum_{\lambda} P^{(1)}\left(\boldsymbol{v}_{N-2}, \boldsymbol{u}_{N} ; \tilde{C}_{N-2}, \ell^{\lambda}\left(\boldsymbol{x}_{1}\right), \mathcal{E}_{m_{1}}\left(\boldsymbol{y}_{2}\right)\right) .
$$

Finally, if $\overrightarrow{\boldsymbol{x}}$ has an additional element $\boldsymbol{x}_{2}$, then this can be accounted for in an upper bound by a final application of Construction $\ell\left(\boldsymbol{x}_{2}\right)$, leading to (7.65).

Proof of Proposition 7.11. We first prove (7.53). For $M=0$, the left-hand side of (7.53) is equal to

$$
\begin{aligned}
\sum_{\left(\boldsymbol{u}_{N}, \boldsymbol{v}_{N}\right)} J_{\boldsymbol{u}_{N}, \boldsymbol{v}_{N}} M_{\boldsymbol{u}_{N}}^{(N+1)}\left(I\left[V_{m_{1}-1}\left(\boldsymbol{v}_{N-1}, \boldsymbol{y}_{2}\right)\right]\right) \delta_{m_{1}, m_{v_{N}}} & \leqslant p \sum_{y_{1}} P^{(N)}\left(\left(y_{1}, m_{1}-1\right) ; V_{m_{1}-1}\left(\boldsymbol{y}_{2}\right)\right) \\
& =p \sum_{y_{1}} R^{(N)}\left(\left(y_{1}, m_{1}-1\right), \boldsymbol{y}_{2}\right),
\end{aligned}
$$

with $R^{(N)}$ defined in (7.17). For $M \geqslant 1$, we use (4.7) and (6.9) to obtain

$$
\Pi_{m_{1}}^{(M)}\left(\boldsymbol{v}_{N} ; \tilde{C}_{N}\right) \leqslant \sum_{\boldsymbol{w}} I\left[\boldsymbol{w} \in \tilde{C}_{N}\right] p \sum_{y_{1}} P^{(M-1)}\left(\boldsymbol{v}_{N},\left(y_{1}, m_{1}-1\right) ; \boldsymbol{w}\right) .
$$

By (7.58), the left-hand side of (7.53) is at most

$$
\begin{aligned}
& p \sum_{\lambda} \sum_{\boldsymbol{w}} \sum_{\left(\boldsymbol{u}_{N}, \boldsymbol{v}_{N}\right)} J_{\boldsymbol{u}_{N}, \boldsymbol{v}_{N}} P^{(N)}\left(\boldsymbol{u}_{N} ; \ell^{\lambda}(\boldsymbol{w}), V_{m_{1}-1}\left(\boldsymbol{y}_{2}\right)\right) \sum_{y_{1}} P^{(M-1)}\left(\boldsymbol{v}_{N},\left(y_{1}, m_{1}-1\right) ; \boldsymbol{w}\right) \\
& \quad \leqslant p \sum_{y_{1}} P^{(N+M)}\left(\left(y_{1}, m_{1}-1\right) ; V_{m_{1}-1}\left(\boldsymbol{y}_{2}\right)\right)=p \sum_{y_{1}} R^{(N+M)}\left(\left(y_{1}, m_{1}-1\right), \boldsymbol{y}_{2}\right),
\end{aligned}
$$

where the inequality has used (6.21) together with the observation that in the application of Construction $V_{m_{1}-1}\left(\boldsymbol{y}_{2}\right)$ to $P^{(N+M)}\left(y_{1}, m_{1}-1\right)$ there are more available lines than if Construction $V_{m_{1}-1}\left(\boldsymbol{y}_{2}\right)$ is applied only to the factor $P^{(N)}\left(\boldsymbol{u}_{N} ; \ell^{\lambda}(\boldsymbol{w}), V_{m_{1}-1}\left(\boldsymbol{y}_{2}\right)\right)$. This proves (7.53).

The proof for (7.54) is the same as the proof of (7.53), with the observation that in (7.57) the order of application of the constructions can be interchanged on the right-hand side.

For (7.55), consider first the case $M=0$. In this case, the left-hand side of (7.55) is equal to

$$
\sum_{\left(\boldsymbol{u}_{N}, \boldsymbol{v}_{N}\right)} J_{\boldsymbol{u}_{N}, \boldsymbol{v}_{N}} M_{\boldsymbol{u}_{N}}^{(N+1)}\left(I\left[\mathcal{E}_{m_{1}}\left(\boldsymbol{v}_{N-1}, \boldsymbol{y}_{2} ; \tilde{C}_{N-1}\right)\right]\right) \delta_{m_{1}, m_{\boldsymbol{v}_{N}}} \leqslant p \sum_{y_{1}} Q^{(N)}\left(\left(y_{1}, m_{1}-1\right), \boldsymbol{y}_{2}\right),
$$


by (7.63) (note that $m_{\boldsymbol{u}_{N}}=m_{1}-1 \leqslant m_{1}$ as required), and with $R^{(N)}$ defined in (7.18). For $M \geqslant 1$, by (7.73), the left-hand side of (7.55) is at most

$$
p \sum_{\boldsymbol{w}} \sum_{\left(\boldsymbol{u}_{N}, \boldsymbol{v}_{N}\right)} J_{\boldsymbol{u}_{N}, \boldsymbol{v}_{N}} M_{\boldsymbol{u}_{N}}^{(N+1)}\left(I\left[\mathcal{E}_{m_{1}}\left(\boldsymbol{v}_{N-1}, \boldsymbol{y}_{2} ; \tilde{C}_{N-1}\right)\right] I\left[\boldsymbol{w} \in \tilde{C}_{N}\right]\right) \sum_{y_{1}} P^{(M-1)}\left(\boldsymbol{v}_{N},\left(y_{1}, m_{1}-1\right) ; \boldsymbol{w}\right) .
$$

The factor $P^{(M-1)}\left(\boldsymbol{v}_{N},\left(y_{1}, m_{1}-1\right)\right.$; $\left.\boldsymbol{w}\right)$ guarantees that $m_{1}-1 \geqslant m_{\boldsymbol{w}}, m_{\boldsymbol{u}_{N}}$, and (7.63) can now be applied to complete the proof of (7.55) as in the proof of (7.53). The proof of (7.56) is similar.

\subsubsection{Proof of (7.19), (7.20) for $K=0$}

We begin with the following lemma. Recall the definitions of the events $H_{m}$ and $V_{m}$ in (5.5) and (7.47).

Lemma 7.15. For $\boldsymbol{v}, \boldsymbol{y} \in \Lambda$ with $m_{\boldsymbol{v}}<m \leqslant m_{\boldsymbol{y}}$,

$$
H_{m}(\boldsymbol{v}, \boldsymbol{y} ;\{\boldsymbol{v}\}) \subseteq V_{m-1}(\boldsymbol{v}, \boldsymbol{y}) .
$$

Proof. Let $b$ denote the last pivotal bond for $\boldsymbol{v} \rightarrow \boldsymbol{y}$, if it exists. The contribution where such a pivotal bond does not exist is equal to $\{\boldsymbol{v} \Rightarrow \boldsymbol{y}\} \subseteq V_{m-1}(\boldsymbol{v}, \boldsymbol{y})$, since $m_{\boldsymbol{v}} \leqslant m-1$. Thus, it suffices to show that

$$
H_{m}(\boldsymbol{v}, \boldsymbol{y} ;\{\boldsymbol{v}\}) \cap\{b \text { last occ. and piv. bond for } \boldsymbol{v} \rightarrow \boldsymbol{y}\}
$$

is a subset of $V_{m-1}(\boldsymbol{v}, \boldsymbol{y})$ for every $b$. When $H_{m}(\boldsymbol{v}, \boldsymbol{y} ;\{\boldsymbol{v}\})$ occurs, $b$ cannot be an $m$-cutting bond for $\boldsymbol{v} \rightarrow \boldsymbol{y}$. Therefore, $m_{\bar{b}} \leqslant m-1$. Since $\bar{b} \Rightarrow \boldsymbol{y}$, this proves that $V_{m-1}(\boldsymbol{v}, \boldsymbol{y})$ occurs with $z$ in (7.47) given by $\boldsymbol{z}=\bar{b}$.

Recall that $\tilde{\mathbb{P}}_{N}$ is the conditional law given that $\left(\boldsymbol{u}_{N}, \boldsymbol{v}_{N}\right)$ is vacant. We also use the following lemma.

Lemma 7.16. Let $F$ be an increasing event, and let $X$ be a non-negative random variable which is independent of the occupation status of the bond $\left(\boldsymbol{u}_{N}, \boldsymbol{v}_{N}\right)$. Then

$$
\widetilde{\mathbb{E}}_{N}(X I[F]) \leqslant \mathbb{E}_{N}(X I[F]) .
$$

Proof. We condition on $\left(\boldsymbol{u}_{N}, \boldsymbol{v}_{N}\right)$ to see that

$$
\mathbb{E}_{N}(X I[F])=J_{\boldsymbol{v}_{N}, \boldsymbol{u}_{N}} \mathbb{E}_{N}\left(X I[F] \mid\left(\boldsymbol{u}_{N}, \boldsymbol{v}_{N}\right) \text { occupied }\right)+\left(1-J_{\boldsymbol{v}_{N}, \boldsymbol{u}_{N}}\right) \mathbb{E}_{N}\left(X I[F] \mid\left(\boldsymbol{u}_{N}, \boldsymbol{v}_{N}\right) \text { vacant }\right) .
$$

By assumption,

$$
\mathbb{E}_{N}\left(X I[F] \mid\left(\boldsymbol{u}_{N}, \boldsymbol{v}_{N}\right) \text { occupied }\right) \geqslant \mathbb{E}_{N}\left(X I[F] \mid\left(\boldsymbol{u}_{N}, \boldsymbol{v}_{N}\right) \text { vacant }\right),
$$

and the claim follows.

Proof of (7.19) for $K=0$. To estimate $\phi_{m_{1}, m_{2}}^{(N, M)}\left(\left\{\boldsymbol{v}_{N-1}\right\}\right)$, we first note that by definition (recall (5.13) and (7.14), (7.15)),

$$
\phi_{m_{1}, m_{2}}^{(N, M)}(A)=\sum_{\left(\boldsymbol{u}_{N}, \boldsymbol{v}_{N}\right)} J_{\boldsymbol{u}_{N}, \boldsymbol{v}_{N}} \sum_{b \neq\left(\boldsymbol{u}_{N}, \boldsymbol{v}_{N}\right)} J_{\underline{b}, \bar{b}} \tilde{M}_{\left(\boldsymbol{u}_{N}, \boldsymbol{v}_{N}\right)}^{(N+1)}\left(I\left[H_{m_{1}}\left(\boldsymbol{v}_{N-1}, \underline{b} ; A\right)\right] \Pi_{m_{1}}^{(M)}\left(\boldsymbol{v}_{N} ; \tilde{C}_{N}\right)\right) \delta_{m_{2}, m_{\bar{b}}} .
$$

We are concerned here with $A=\left\{\boldsymbol{v}_{N-1}\right\}$. By Lemma 7.15,

$$
H_{m_{1}}\left(\boldsymbol{v}_{N-1}, \underline{b} ;\left\{\boldsymbol{v}_{N-1}\right\}\right) \subseteq V_{m_{1}-1}\left(\boldsymbol{v}_{N-1}, \underline{b}\right) .
$$

The event $V_{m_{1}-1}\left(\boldsymbol{v}_{N-1}, \underline{b}\right)$ is increasing, and the event $E_{N}^{\prime}=E^{\prime}\left(\boldsymbol{v}_{N-1}, \boldsymbol{u}_{N} ; \tilde{C}_{N-1}\right)$ does not depend on the occupation status of $\left(\boldsymbol{u}_{N}, \boldsymbol{v}_{N}\right)$. Also, the random variable $\Pi_{m_{1}}^{(M)}\left(\boldsymbol{v}_{N} ; \tilde{C}_{N}\right)$ is independent of the occupation status of $\left(\boldsymbol{u}_{N}, \boldsymbol{v}_{N}\right)$, since $\tilde{C}_{N}=\tilde{C}_{N}^{\left(\boldsymbol{u}_{N}, \boldsymbol{v}_{N}\right)}\left(\boldsymbol{v}_{N-1}\right)$ is. Therefore, by Lemma 7.16, the $\widetilde{\mathbb{E}}_{N}$ expectation in $\widetilde{M}_{\left(\boldsymbol{u}_{N}, \boldsymbol{v}_{N}\right)}^{(N+1)}$ implicit in (7.82) can be bounded above by the corresponding expectation without the tilde, i.e.,

$$
\widetilde{\mathbb{E}}_{N}\left(I\left[E_{N}^{\prime} \cap V_{m_{1}-1}\left(\boldsymbol{v}_{N-1}, \underline{b}\right)\right] \Pi_{m_{1}}^{(M)}\left(\boldsymbol{v}_{N} ; \tilde{C}_{N}\right)\right) \leqslant \mathbb{E}_{N}\left(I\left[E_{N}^{\prime} \cap V_{m_{1}-1}\left(\boldsymbol{v}_{N-1}, \underline{b}\right)\right] \Pi_{m_{1}}^{(M)}\left(\boldsymbol{v}_{N} ; \tilde{C}_{N}\right)\right) .
$$

Omitting the restriction $b \neq\left(\boldsymbol{u}_{N}, \boldsymbol{v}_{N}\right)$, it follows that 


$$
\begin{aligned}
\phi_{m_{1}, m_{2}}^{(N, M, 0)}\left(\left\{\boldsymbol{v}_{N-1}\right\}\right) & \leqslant \sum_{b} J_{\underline{b}, \bar{b}} \sum_{\left(\boldsymbol{u}_{N}, \boldsymbol{v}_{N}\right)} J_{\boldsymbol{u}_{N}, \boldsymbol{v}_{N}} M_{\boldsymbol{u}_{N}}^{(N+1)}\left(I\left[V_{m_{1}-1}\left(\boldsymbol{v}_{N-1}, \underline{b}\right)\right] \Pi_{m_{1}}^{(M)}\left(\boldsymbol{v}_{N} ; \tilde{C}_{N}\right)\right) \delta_{m_{2}, m_{\bar{b}}} \\
& =p \sum_{y_{2}} \sum_{\left(\boldsymbol{u}_{N}, \boldsymbol{v}_{N}\right)} J_{\boldsymbol{u}_{N}, \boldsymbol{v}_{N}} M_{\boldsymbol{u}_{N}}^{(N+1)}\left(I\left[V_{m_{1}-1}\left(\boldsymbol{v}_{N-1},\left(y_{2}, m_{2}-1\right)\right)\right] \Pi_{m_{1}}^{(M)}\left(\boldsymbol{v}_{N} ; \tilde{C}_{N}\right)\right),
\end{aligned}
$$

where we have written $\underline{b}=\left(y_{2}, m_{2}-1\right)$ and have used $\sum_{\bar{b}} J_{\underline{b}, \bar{b}}=p$. The bound (7.19) for $K=0$ now follows from (7.53).

Next, we prepare for the proof of (7.20) for $K=0$. Let $N \geqslant 1$. To partition the event $H_{m}\left(\boldsymbol{v}_{N-1}, \underline{b} ; \tilde{C}_{N-1}\right)$ occurring in (7.82) (for $A=\tilde{C}_{N-1}$ ), we define

$$
\begin{aligned}
& G_{m}^{(1)}\left(\boldsymbol{v}_{N-1}, \underline{b}\right)=H_{m}\left(\boldsymbol{v}_{N-1}, \underline{b} ; \tilde{C}_{N-1}\right) \cap V_{m-1}\left(\boldsymbol{v}_{N-1}, \underline{b}\right), \\
& G_{m}^{(2)}\left(\boldsymbol{v}_{N-1}, \underline{b}\right)=H_{m}\left(\boldsymbol{v}_{N-1}, \underline{b} ; \tilde{C}_{N-1}\right) \cap V_{m-1}\left(\boldsymbol{v}_{N-1}, \underline{b}\right)^{c} .
\end{aligned}
$$

In terms of these events, $H_{m}\left(\boldsymbol{v}_{N-1}, \underline{b} ; \tilde{C}_{N-1}\right)$ is given by the disjoint union

$$
H_{m}\left(\boldsymbol{v}_{N-1}, \underline{b} ; \tilde{C}_{N-1}\right)=G_{m}^{(1)}\left(\boldsymbol{v}_{N-1}, \underline{b}\right) \dot{\cup} G_{m}^{(2)}\left(\boldsymbol{v}_{N-1}, \underline{b}\right) .
$$

Clearly,

$$
G_{m}^{(1)}\left(\boldsymbol{v}_{N-1}, \underline{b}\right) \subseteq V_{m-1}\left(\boldsymbol{v}_{N-1}, \underline{b}\right) .
$$

For $G_{m}^{(2)}\left(\boldsymbol{v}_{N-1}, \underline{b}\right)$, we will use the following lemma. Recall that the event $\mathcal{E}_{m}$ was defined in (7.48).

Lemma 7.17. For $\boldsymbol{v}_{N-1}, \boldsymbol{y}_{2} \in \Lambda$ and $m \geqslant 1$ such that $m_{\boldsymbol{v}_{N-1}}<m \leqslant m_{\boldsymbol{y}_{2}}$,

$$
G_{m}^{(2)}\left(\boldsymbol{v}_{N-1}, \boldsymbol{y}_{2}\right) \subseteq \mathcal{E}_{m}\left(\boldsymbol{v}_{N-1}, \boldsymbol{y}_{2} ; \tilde{C}_{N-1}\right) .
$$

Proof. Suppose that $G_{m}^{(2)}\left(\boldsymbol{v}_{N-1}, \boldsymbol{y}_{2}\right)$ occurs. It cannot be the case that $\boldsymbol{v}_{N-1} \Rightarrow \boldsymbol{y}_{2}$, since this contributes instead to $G_{m}^{(1)}\left(\boldsymbol{v}_{N-1}, \boldsymbol{y}_{2}\right)$. Thus there is at least one occupied pivotal bond for $\boldsymbol{v}_{N-1} \rightarrow \boldsymbol{y}_{2}$, and we denote the last such bond by $b$. Then $\left\{\boldsymbol{v}_{N-1} \rightarrow \bar{b} \Rightarrow \boldsymbol{y}_{2}\right\}$. It must be the case that $m_{\bar{b}}>m-1$, since otherwise $G_{m}^{(1)}\left(\boldsymbol{v}_{N-1}, \boldsymbol{y}_{2}\right)$ occurs. By the definition of $H_{m}\left(\boldsymbol{v}_{N-1}, \underline{b} ; \tilde{C}_{N-1}\right)$ in (5.5), $b$ cannot be $m$-cutting for $\boldsymbol{v}_{N-1} \stackrel{\tilde{C}_{N-1}}{\longrightarrow} \boldsymbol{y}_{2}$, and hence $\left\{\boldsymbol{v}_{N-1} \stackrel{\tilde{C}_{N-1}}{\longrightarrow} \underline{b}\right\}^{c}$ occurs. Since $\boldsymbol{v}_{N-1} \stackrel{\tilde{C}_{N-1}}{\longrightarrow} \boldsymbol{y}_{2}$ occurs by (5.5), we conclude that $\bar{b} \stackrel{\tilde{C}_{N-1}}{\longrightarrow} \boldsymbol{y}_{2}$. Therefore, $\mathcal{E}_{m}\left(\boldsymbol{v}_{N-1}, \boldsymbol{y}_{2} ; \tilde{C}_{N-1}\right)$ occurs, with $z=\bar{b}$ in (7.48).

Proof of (7.20) for $K=0$. Let $N \geqslant 1$. We estimate (7.82) for $A=\tilde{C}_{N-1}$, using the partition (7.88) for the event $H_{m}\left(\boldsymbol{v}_{N-1}, \underline{b} ; \tilde{C}_{N-1}\right)$.

Consider first the contribution due to $G_{m_{1}}^{(1)}\left(\boldsymbol{v}_{N-1}, \underline{b}\right)$. The inclusion (7.89) plays a role identical to the inclusion (7.83), and the analysis of (7.84), (7.85) then applies in an identical fashion to give the first term on the right-hand side of (7.20) as an upper bound for this contribution.

It remains to show that the contribution to (7.82) due to $G_{m_{1}}^{(2)}\left(\boldsymbol{v}_{N-1}, \underline{b}\right)$, namely

$$
\sum_{b} J_{\underline{b}, \bar{b}} \sum_{\left(\boldsymbol{u}_{N}, \boldsymbol{v}_{N}\right)} J_{\boldsymbol{u}_{N}, \boldsymbol{v}_{N}} \tilde{M}_{\left(\boldsymbol{u}_{N}, \boldsymbol{v}_{N}\right)}^{(N+1)}\left(I\left[G_{m_{1}}^{(2)}\left(\boldsymbol{v}_{N-1}, \underline{b}\right)\right] \Pi_{m_{1}}^{(M)}\left(\boldsymbol{v}_{N} ; \tilde{C}_{N}\right)\right) \delta_{m_{2}, m_{\bar{b}}},
$$

is bounded by the second term on the right-hand side of (7.20). By Lemma 7.17,

$$
I\left[G_{m_{1}}^{(2)}\left(\boldsymbol{v}_{N-1}, \underline{b}\right)\right] \leqslant I\left[\mathcal{E}_{m_{1}}\left(\boldsymbol{v}_{N-1}, \underline{b} ; \tilde{C}_{N-1}\right)\right] .
$$

Since $\mathcal{E}_{m_{1}}\left(\boldsymbol{v}_{N-1}, \underline{b} ; \tilde{C}_{N-1}\right)$ is increasing, we may apply Lemma 7.16 as before to conclude that the $\widetilde{\mathbb{E}}_{N}$ expectation in $\tilde{M}_{\left(\boldsymbol{u}_{N}, \boldsymbol{v}_{N}\right)}^{(N+1)}$ implicit in (7.91) obeys

$$
\widetilde{\mathbb{E}}_{N}\left(I\left[E_{N}^{\prime} \cap G_{m}^{(2)}\left(\boldsymbol{v}_{N-1}, \underline{b}\right)\right] \Pi_{m_{1}}^{(M)}\left(\boldsymbol{v}_{N} ; \tilde{C}_{N}\right)\right) \leqslant \mathbb{E}_{N}\left(I\left[E_{N}^{\prime} \cap \mathcal{E}_{m_{1}}\left(\boldsymbol{v}_{N-1}, \underline{b} ; \tilde{C}_{N-1}\right)\right] \Pi_{m_{1}}^{(M)}\left(\boldsymbol{v}_{N} ; \tilde{C}_{N}\right)\right),
$$

and hence

$$
\tilde{M}_{\left(\boldsymbol{u}_{N}, \boldsymbol{v}_{N}\right)}^{(N+1)}\left(I\left[G_{m_{1}}^{(2)}\left(\boldsymbol{v}_{N-1}, \underline{b}\right)\right] \Pi_{m_{1}}^{(M)}\left(\boldsymbol{v}_{N} ; \tilde{C}_{N}\right)\right) \leqslant M_{\boldsymbol{u}_{N}}^{(N+1)}\left(I\left[\mathcal{E}_{m_{1}}\left(\boldsymbol{v}_{N-1}, \underline{b} ; \tilde{C}_{N-1}\right)\right] \Pi_{m_{1}}^{(M)}\left(\boldsymbol{v}_{N} ; \tilde{C}_{N}\right)\right) .
$$


It follows that the contribution to (7.82) due to $G^{(2)}$ is bounded above by

$$
\begin{aligned}
& \sum_{b} J_{\underline{b}, \bar{b}} \sum_{\left(\boldsymbol{u}_{N}, \boldsymbol{v}_{N}\right)} J_{\boldsymbol{u}_{N}, \boldsymbol{v}_{N}} M_{\boldsymbol{u}_{N}}^{(N+1)}\left(I\left[\mathcal{E}_{m_{1}}\left(\boldsymbol{v}_{N-1}, \underline{b} ; \tilde{C}_{N-1}\right)\right] \Pi_{m_{1}}^{(M)}\left(\boldsymbol{v}_{N} ; \tilde{C}_{N}\right)\right) \delta_{m_{2}, m_{\bar{b}}} \\
& \quad=p \sum_{y_{2}} \sum_{\left(\boldsymbol{u}_{N}, \boldsymbol{v}_{N}\right)} J_{\boldsymbol{u}_{N}, \boldsymbol{v}_{N}} M_{\boldsymbol{u}_{N}}^{(N+1)}\left(I\left[\mathcal{E}_{m_{1}}\left(\boldsymbol{v}_{N-1},\left(y_{2}, m_{2}-1\right) ; \tilde{C}_{N-1}\right)\right] \Pi_{m_{1}}^{(M)}\left(\boldsymbol{v}_{N} ; \tilde{C}_{N}\right)\right),
\end{aligned}
$$

where we have written $\underline{b}=\left(y_{2}, m_{2}-1\right)$ and have used $\sum_{\bar{b}} J_{\underline{b}, \bar{b}}=p$. The bound (7.20) now follows from Proposition 7.11.

\subsubsection{Proof of (7.19)-(7.20) for $K \geqslant 1$}

We first prove (7.19) for $K=1$. For this, we note that

$$
\phi_{m_{1}, m_{2}}^{(N, M, 1)}(A)=\sum_{b} J_{\underline{b}, \bar{b}} \sum_{\left(\boldsymbol{u}_{N}, \boldsymbol{v}_{N}\right)} J_{\boldsymbol{u}_{N}, \boldsymbol{v}_{N}} \tilde{M}_{\left(\boldsymbol{u}_{N}, \boldsymbol{v}_{N}\right)}^{(N+1)}\left(I\left[H_{m_{1}}\left(\boldsymbol{v}_{N-1}, \underline{b} ; A\right)\right] \Pi_{m_{2}}^{(1)}\left(\bar{b} ; \tilde{C}_{N}^{b}\right) \Pi_{m_{1}}^{(M)}\left(\boldsymbol{v}_{N} ; \tilde{C}_{N}\right)\right) .
$$

By (6.9) and (7.14),

$$
\Pi_{m_{2}}^{(1)}\left(\bar{b} ; \tilde{C}_{N}^{b}\right) \leqslant p \sum_{y_{2}} P_{m_{2}}^{(0)}\left(\bar{b},\left(y_{2}, m_{2}-1\right) ; \tilde{C}_{N}^{b}\right)=p \sum_{\boldsymbol{a}} I\left[\boldsymbol{a} \in \tilde{C}_{N}^{b}\right] \sum_{y_{2}} P^{(0)}\left(\bar{b},\left(y_{2}, m_{2}-1\right) ; \boldsymbol{a}\right) .
$$

It then follows from (7.83), Lemma 7.16 and (7.54) that

$$
\phi_{m_{1}, m_{2}}^{(N, M, 1)}\left(\left\{\boldsymbol{v}_{N-1}\right\}\right) \leqslant p^{2} \sum_{y_{1}, y_{2}} \sum_{\boldsymbol{a}} \sum_{b} J_{\underline{b}, \bar{b}} R^{(N+M)}\left(\left(y_{1}, m_{1}-1\right), \underline{b} ; \ell(\boldsymbol{a})\right) P^{(0)}\left(\bar{b},\left(y_{2}, m_{2}-1\right) ; \boldsymbol{a}\right) .
$$

By (7.11), Construction $\ell(\boldsymbol{a})$ followed by multiplication by $J_{b, \bar{b}} P^{(0)}\left(\bar{b},\left(y_{2}, m_{2}-1\right) ; \boldsymbol{a}\right)$ and by summation over $\boldsymbol{a}$ and $b$ is equivalent to Construction $E$. This shows that

$$
\phi_{m_{1}, m_{2}}^{(N, M, 1)}\left(\left\{\boldsymbol{v}_{N-1}\right\}\right) \leqslant p^{2} \sum_{y_{1}, y_{2}} R_{m_{1}-1, m_{2}-1}^{(N, M, 1)}\left(y_{1}, y_{2}\right),
$$

which proves (7.19) for $K=1$.

To extend the proof of (7.19) to $K \geqslant 1$, we estimate $\Pi_{m_{2}}^{(K)}\left(\bar{b} ; \tilde{C}_{N}^{b}\right)$ using (6.9) and (7.14). According to Remark 7.4, the resulting bound on $\Pi_{m_{2}}^{(K)}\left(\bar{b} ; \tilde{C}_{N}^{b}\right)$ is the same as $K-1$ applications of Construction $E$ to $p \sum_{x} P^{(0)}\left(\bar{b},\left(x, m_{2}-\right.\right.$ $1) ; \tilde{C}_{N}^{b}$ ). Therefore, by definition of $R_{m_{1}, m_{2}}^{(N, M)}\left(y_{1}, y_{2}\right)$, (7.19) for $K \geqslant 1$ follows from (7.19) for $K=1$.

The proof of (7.20) proceeds similarly, with (7.56) used in place of (7.54).

\subsubsection{Proof of final statement of Proposition 7.6}

Finally, we suppose that $\left(m_{1}, m_{2}\right) \neq(1,1)$, and show that in this case $\phi_{m_{1}, m_{2}}^{(0,0,0)}(\{\mathbf{0}\}) \leqslant C \beta b_{m_{1}, m_{2}}$.

We have already seen in (7.19) and (7.21) that $\phi_{m_{1}, m_{2}}^{(0,0,0)}(\{\boldsymbol{0}\}) \leqslant C b_{m_{1}, m_{2}}$, and the only issue is to establish that a factor $\beta$ can be inserted into this upper bound. A factor $\beta$ arises in diagrammatic estimates when the bound (7.24) is used to estimate $\left\|\tau_{m}\right\|_{\infty}$ with $m \geqslant 1$. According to (7.17), the diagrammatic upper bound $p_{c}^{2} \sum_{y_{1}, y_{2}} R_{m_{1}-1, m_{2}-1}^{(0,0,0)}\left(y_{1}, y_{2}\right)$ on $\phi_{m_{1}, m_{2}}^{(0,0,0)}(\{\mathbf{0}\})$ given by (7.19) is equal to

$$
\sum_{v} \sum_{z} P_{m_{1}-1}^{(0)}\left(y_{1} ; \ell\left(v, m_{1}-1\right), 1(z)\right) \tau_{m_{2}-m_{1}}\left(y_{2}-v\right) \tau_{m_{2}-1-m_{z}}\left(y_{2}-z\right) .
$$

When $\left(m_{1}, m_{2}\right) \neq(1,1)$, there is at least one line with length greater than zero, and a factor $\beta$ then results from standard estimates.

\section{Diagrammatic estimates: Bounds for $e_{n+1}$}

In this section, we prove the bound (1.19) on $e_{n+1}\left(p_{c}\right)$. We assume throughout that

$$
\theta_{m}\left(p_{c}\right) \leqslant C_{\theta}(m+1)^{-1} \text { for all } 0 \leqslant m \leqslant n,
$$


which is a hypothesis of Theorem 1.1(ii). Throughout this section, we fix $d>4, p=p_{c}$, and consider the spread-out model with $\beta$ sufficiently small, although some of our arguments apply more generally. Note that we can extend (8.1) to $m=n+1$ by conditioning on a vertex at time 1 and using the Markov property and (1.9) to obtain

$$
\theta_{n+1}\left(p_{c}\right) \leqslant \sum_{v \in \mathbb{Z}^{d}} p_{c} D(v) \theta_{n}\left(p_{c}\right)=p_{c} \theta_{n}\left(p_{c}\right) \leqslant 2 C_{\theta}(n+1)^{-1} \leqslant 4 C_{\theta}(n+2)^{-1} .
$$

We absorb the constant 4 into $C_{\theta}$ and regard (8.1) as applying also when $m=n+1$ in what follows.

In Section 8.1, we reduce (1.19) to bounds on $e_{n+1}^{(N)}(1), \ldots, e_{n+1}^{(N)}(5)$. In Section 8.2, we provide some preliminary convolution bounds. Then we estimate the five error terms in order of increasing difficulty, namely $e_{n+1}^{(N)}(3), e_{n+1}^{(N)}(5)$, $e_{n+1}^{(N)}(2), e_{n+1}^{(N)}(4), e_{n+1}^{(N)}(1)$, in Sections 8.3-8.7, respectively.

\subsection{Reduction of proof of (1.19)}

Let

$$
\Delta_{n}^{\prime}= \begin{cases}(n+1)^{(6-d) / 2} & (4<d<6), \\ \log n & (d=6), \\ 1 & (d>6) .\end{cases}
$$

The following proposition will be proved in Sections 8.3-8.7.

Proposition 8.1 (Bounds on error terms). Let $d>4, p=p_{c}$, for the spread-out model with $\beta$ sufficiently small. Let $n \geqslant 1$ and $N \geqslant 0$, and assume that $\theta_{m}\left(p_{c}\right) \leqslant C_{\theta}(m+1)^{-1}$ for all $0 \leqslant m \leqslant n$. Then

$$
\begin{aligned}
& \left|e_{n+1}^{(N)}(1)\right| \leqslant C C_{\theta}^{3}(n+1)^{-3} \delta_{N, 0}+C_{\theta}^{3}(C \beta)^{N \vee 1}\left[(n+1)^{-d / 2} \log n+(n+1)^{-3} \Delta_{n+1}^{\prime}\right], \\
& \left|e_{n+1}^{(N)}(2)\right| \leqslant C C_{\theta}^{3}(n+1)^{-3} \delta_{N, 0}+C_{\theta}^{3}(C \beta)^{N \vee 1}\left[(n+1)^{-d / 2} \log n+(n+1)^{-3} \Delta_{n+1}^{\prime}\right], \\
& \left|e_{n+1}^{(N)}(3)\right| \leqslant C C_{\theta}(C \beta)^{N \vee 1}(n+1)^{-d / 2} \log n, \\
& \left|e_{n+1}^{(N)}(4)\right| \leqslant C C_{\theta}^{3}(n+1)^{-3} \delta_{N, 0}+C_{\theta}^{3}(C \beta)^{N \vee 1}\left[(n+1)^{-d / 2} \log n+(n+1)^{-3} \Delta_{n+1}^{\prime}\right], \\
& \left|e_{n+1}^{(N)}(5)\right| \leqslant C C_{\theta}^{3}(n+1)^{-3} \delta_{N, 0}+C_{\theta}^{3}(C \beta)^{N \vee 1}\left[(n+1)^{-d / 2} \log n+(n+1)^{-3} \Delta_{n+1}^{\prime}\right] .
\end{aligned}
$$

Proof of (1.19) subject to Proposition 8.1. It follows from (5.19) that $\left|e_{n+1}\right|$ is bounded above by the sum of the right-hand sides of (8.4)-(8.8). The elementary inequalities $(n+1)^{-3} \Delta_{n+1}^{\prime} \leqslant(n+1)^{-2} \Delta_{n+1}$ and $(n+1)^{-d / 2} \log n \leqslant$ $(n+1)^{-2} \Delta_{n+1}$ then yield (1.19) (recall (1.16)).

\subsection{Convolution bounds}

This section contains several convolution bounds to be used in Sections 8.3-8.7.

Lemma 8.2. Let $d \geqslant 1$ and suppose that $F_{m}$ obeys $F_{m} \leqslant C_{F}(m+1)^{-d / 2}$. If (8.1) holds, then

$$
\sum_{m=\lfloor(n+1) / 2\rfloor+1}^{n+1} F_{m} \theta_{n+1-m} \leqslant C C_{\theta} C_{F}(n+1)^{-d / 2} \log n,
$$

where $C$ is a constant that only depends on $d$.

Proof. We use the two assumptions to obtain the upper bound

$$
\begin{aligned}
\sum_{m=\lfloor(n+1) / 2\rfloor+1}^{n+1} C_{F}(m+1)^{-d / 2} C_{\theta}(n-m+2)^{-1} & \leqslant C C_{\theta} C_{F}(n+1)^{-d / 2} \sum_{m=\lfloor(n+1) / 2\rfloor+1}^{n+1}(n-m+2)^{-1} \\
& \leqslant C C_{\theta} C_{F}(n+1)^{-d / 2} \log n .
\end{aligned}
$$


Let $F_{m}\left(\theta_{n+1}, \theta_{n+1}\right)$ denote the result of two applications of Construction $\theta_{n+1}$ to $F_{m}$. (According to Definition 6.5, each of the two added connections to $n$ is added to lines originally in $F_{m}$, i.e., the second added connection to $n$ cannot be attached to the first added connection to $n$.)

Lemma 8.3. Let $d>4$ and suppose that $F_{m}$ is a diagram such that $F_{m} \leqslant C_{F}(m+1)^{-d / 2}$. Suppose that $F_{m}$ contains at most $\mathcal{L}$ lines at any fixed time, that the largest time coordinate of a vertex in $F_{m}$ is $m$, and assume (8.1). Then

$$
\sum_{m=0}^{\lfloor n / 2\rfloor} F_{m}\left(\theta_{n+1}, \theta_{n+1}\right) \theta_{n-m} \leqslant C C_{\theta}^{3} C_{F} \mathcal{L}^{2}(n+1)^{-3} \Delta_{n+1}^{\prime},
$$

where $C$ is a constant that only depends on $d$.

Proof. Each factor $\theta$ appearing in $F_{m}\left(\theta_{n+1}, \theta_{n+1}\right) \theta_{n-m}$ has subscript at least $n-\lfloor n / 2\rfloor$. Therefore, by (8.1),

$$
\sum_{m=0}^{\lfloor n / 2\rfloor} F_{m}\left(\theta_{n+1}, \theta_{n+1}\right) \theta_{n-m} \leqslant C C_{\theta}^{3}(n+1)^{-3} \sum_{m=0}^{\lfloor n / 2\rfloor} \sum_{l_{1}, l_{2}=0}^{m} F_{m}\left(1\left(l_{1}\right), 1\left(l_{2}\right)\right) \text {. }
$$

By Lemma 7.8(a), for each $l_{1}, l_{2}$, we have

$$
F_{m}\left(1\left(l_{1}\right), 1\left(l_{2}\right)\right) \leqslant C C_{F} \mathcal{L}^{2}(m+1)^{-d / 2} .
$$

Therefore,

$$
\sum_{m=0}^{\lfloor n / 2\rfloor} F_{m}\left(\theta_{n+1}, \theta_{n+1}\right) \theta_{n-m} \leqslant C C_{\theta}^{3} C_{F} \mathcal{L}^{2}(n+1)^{-3} \sum_{m=0}^{\lfloor n / 2\rfloor}(m+1)^{-(d-4) / 2} .
$$

Performing the sum gives

$$
\sum_{m=0}^{\lfloor n / 2\rfloor}(m+1)^{-(d-4) / 2} \leqslant C \Delta_{n+1}^{\prime},
$$

and this completes the proof.

Corollary 8.4. Let $d>4$ and suppose that $F_{m}$ is a diagram such that $F_{m} \leqslant C_{F}(m+1)^{-d / 2}$. Suppose that $F_{m}$ contains at most $\mathcal{L}$ lines at any fixed time, that the largest time coordinate of a vertex in $F_{m}$ is $m$, and assume (8.1). Then

$$
\sum_{m=0}^{n}\left[F_{m}\left(\theta_{n+1}, \theta_{n+1}\right) \wedge F_{m}\right] \theta_{n-m} \leqslant C C_{\theta}^{3} C_{F}\left[\mathcal{L}^{2}(n+1)^{-3} \Delta_{n+1}^{\prime}+(n+1)^{-d / 2} \log n\right],
$$

where $C$ is a constant that only depends on $d$.

Proof. For $m \leqslant\left\lfloor\frac{n}{2}\right\rfloor$ we use Lemma 8.3, and for $m \geqslant\left\lfloor\frac{n}{2}\right\rfloor+1$ we use Lemma 8.2. Adding the two contributions proves the claim (we assume that $C_{\theta} \geqslant 1$ ).

Let $F_{m}\left(\theta_{n+1}\right)$ denote the result of an application of Construction $\theta_{n+1}$ to $F_{m}$.

Lemma 8.5. Let $d>4$ and suppose that $F_{m}$ is a diagram such that $F_{m} \leqslant C_{F}(m+1)^{-d / 2}$. Suppose that $F_{m}$ contains at most $\mathcal{L}$ lines at any fixed time, that the largest time coordinate of a vertex in $F_{m}$ is $m$, and assume (8.1). Then

$$
\sum_{m=0}^{\lfloor n / 2\rfloor} F_{m}\left(\theta_{n+1}\right) \theta_{n-m}^{2} \leqslant C C_{\theta}^{3} C_{F} \mathcal{L}(n+1)^{-3},
$$

where $C$ is a constant that only depends on $d$.

Proof. The proof is a straightforward adaptation of the proof of Lemma 8.3. Each factor $\theta$ appearing in $F_{m}\left(\theta_{n+1}\right) \theta_{n-m}^{2}$ has subscript at least $n-\lfloor n / 2\rfloor$. Therefore, by (8.1), 


$$
\sum_{m=0}^{\lfloor n / 2\rfloor} F_{m}\left(\theta_{n+1}\right) \theta_{n-m}^{2} \leqslant C C_{\theta}^{3}(n+1)^{-3} \sum_{m=0}^{\lfloor n / 2\rfloor} \sum_{l_{1}=0}^{m} F_{m}\left(1\left(l_{1}\right)\right) .
$$

By Lemma 7.8(a),

$$
\sum_{l_{1}=0}^{m} F_{m}\left(1\left(l_{1}\right)\right) \leqslant C C_{F} \mathcal{L}(m+1)^{-(d-2) / 2},
$$

and the claim follows from (8.15).

Lemma 8.6. Let $d>4$ and suppose that $F_{m_{1}, m_{2}}$ is a diagram such that $F_{m_{1}, m_{2}} \leqslant C_{F} b_{m_{1}, m_{2}}$. Suppose that $F_{m_{1}, m_{2}}$ contains at most $\mathcal{L}$ lines at any fixed time, that the largest time coordinate of a vertex in $F_{m_{1}, m_{2}}$ is $m_{1} \vee m_{2}$, and assume (8.1). Then

$$
\begin{aligned}
& \sum_{m_{1}=0}^{\lfloor(n+1) / 2\rfloor} \sum_{m_{2}=m_{1}}^{n+1}\left[F_{m_{1}, m_{2}} \wedge F_{m_{1}, m_{2}}\left(\theta_{n+1}\right)\right] \theta_{n+1-m_{1}} \theta_{n+1-m_{2}} \\
& \quad \leqslant C C_{\theta}^{3} C_{F} \mathcal{L}\left[(n+1)^{-d / 2} \log n+(n+1)^{-3} \Delta_{n+1}^{\prime}\right],
\end{aligned}
$$

where $C$ is a constant that only depends on $d$.

Proof. Since $m_{1} \leqslant\lfloor(n+1) / 2\rfloor,(8.1)$ implies that $\theta_{n+1-m_{1}} \leqslant C C_{\theta}(n+1)^{-1}$. We divide the sum over $m_{2}$ according to whether $\lfloor 3 n / 4\rfloor+1 \leqslant m_{2} \leqslant n+1$ or $m_{1} \leqslant m_{2} \leqslant\lfloor 3 n / 4\rfloor$.

Suppose first that $\lfloor 3 n / 4\rfloor+1 \leqslant m_{2} \leqslant n+1$. In this case,

$$
b_{m_{1}, m_{2}} \leqslant C\left(m_{1}+1\right)^{-(d-2) / 2}(n+1)^{-(d-2) / 2} .
$$

We bound the minimum in (8.20) by $F_{m_{1}, m_{2}}$ to see that this case contributes at most

$$
C C_{\theta}^{2} C_{F}(n+1)^{-d / 2} \sum_{m_{1}=0}^{\lfloor(n+1) / 2\rfloor}\left(m_{1}+1\right)^{-(d-2) / 2} \sum_{m_{2}=\lfloor 3 n / 4\rfloor+1}^{n+1}\left(n+2-m_{2}\right)^{-1} \leqslant C C_{\theta}^{2} C_{F}(n+1)^{-d / 2} \log n
$$

to the left-hand side of (8.20).

In the remaining case, we have $m_{1} \leqslant m_{2} \leqslant\lfloor 3 n / 4\rfloor$, and hence $\theta_{n+1-m_{2}} \leqslant C C_{\theta}(n+1)^{-1}$. We bound the minimum in (8.20) by $F_{m_{1}, m_{2}}\left(\theta_{n+1}\right)$ to see that this case contributes at most

$$
\sum_{m_{1}=0}^{\lfloor(n+1) / 2\rfloor} \sum_{m_{2}=m_{1}}^{\lfloor 3 n / 4\rfloor} F_{m_{1}, m_{2}}\left(\theta_{n+1}\right) \theta_{n+1-m_{1}} \theta_{n+1-m_{2}} \leqslant C C_{\theta}^{2}(n+1)^{-2} \sum_{m_{1}=0}^{\lfloor(n+1) / 2\rfloor} \sum_{m_{2}=m_{1}}^{\lfloor 3 n / 4\rfloor} F_{m_{1}, m_{2}}\left(\theta_{n+1}\right) .
$$

By Lemma 7.8(a) and the fact that $m_{2} \leqslant\lfloor 3 n / 4\rfloor$,

$$
F_{m_{1}, m_{2}}\left(\theta_{n+1}\right)=\sum_{l=0}^{m_{2}} F_{m_{1}, m_{2}}(1(l)) \theta_{n+1-l} \leqslant C C_{\theta}(n+1)^{-1} \mathcal{L}\left(m_{2}+1\right) C_{F} b_{m_{1}, m_{2}} .
$$

Therefore,

$$
\sum_{m_{1}=0}^{\lfloor(n+1) / 2\rfloor} \sum_{m_{2}=m_{1}}^{\lfloor 3 n / 4\rfloor} F_{m_{1}, m_{2}}\left(\theta_{n+1}\right) \theta_{n+1-m_{1}} \theta_{n+1-m_{2}} \leqslant C C_{\theta}^{3} C_{F} \mathcal{L}(n+1)^{-3} \sum_{m_{1}=0}^{\lfloor(n+1) / 2\rfloor} \sum_{m_{2}=m_{1}}^{\lfloor 3 n / 4\rfloor}\left(m_{2}+1\right) b_{m_{1}, m_{2}} .
$$

It follows from (8.15) that

$$
\sum_{m_{1}=0}^{\lfloor(n+1) / 2\rfloor} \sum_{m_{2}=m_{1}}^{\lfloor 3 n / 4\rfloor}\left(m_{2}+1\right) b_{m_{1}, m_{2}} \leqslant \sum_{m_{1}=0}^{\lfloor(n+1) / 2\rfloor} \sum_{m_{2}=m_{1}}^{\lfloor 3 n / 4\rfloor}\left[\left(m_{1}+1\right)+\left(m_{2}-m_{1}+1\right)\right] b_{m_{1}, m_{2}} \leqslant C \Delta_{n+1}^{\prime},
$$

and this completes the proof. 
It is a consequence of their definitions in (7.17), (7.18) and the fact that $P^{(N)}(y)$ has at most 4 lines at any fixed time that the diagrams $R^{(N)}\left(\boldsymbol{y}_{1}, \boldsymbol{y}_{2}\right)$ and $Q^{(N)}\left(\boldsymbol{y}_{1}, \boldsymbol{y}_{2}\right)$ have at most $\mathcal{L}=6$ lines at any fixed time. Also, $R^{(N, M, K)}\left(\boldsymbol{y}_{1}, \boldsymbol{y}_{2}\right)$ and $Q^{(N, M, K)}\left(\boldsymbol{y}_{1}, \boldsymbol{y}_{2}\right)$ have $\mathcal{L}=7$. For this reason, factors of $\mathcal{L}$ will be unimportant in our applications of the above lemmas.

\subsection{Bound on $e_{n+1}^{(N)}(3)$}

Proof of (8.6). Fix $N \geqslant 0$. It is an immediate consequence of (4.31) and (4.29) that

$$
\left|e_{n+1}^{(N)}(3)\right| \leqslant \sum_{m_{1}=\lfloor(n+1) / 2\rfloor+1}^{n+1}\left[\left|\kappa_{m_{1}, n+1}^{(N)}\left(\left\{\boldsymbol{v}_{N-1}\right\}\right)\right|+\left|\kappa_{m_{1}, n+1}^{(N)}\left(\tilde{C}_{N-1}\right)\right|\right] p_{c} \theta_{n+1-m_{1}} .
$$

In the definition (4.18) of $\kappa_{m_{1}, n+1}^{(N)}(A)$, we estimate the indicator function by 1 to obtain

$$
\left|\kappa_{m_{1}, n+1}^{(N)}(A)\right| \leqslant \sum_{M=0}^{\infty} \sum_{\left(\boldsymbol{u}_{N}, \boldsymbol{v}_{N}\right)} J_{\boldsymbol{u}_{N}, \boldsymbol{v}_{N}} M_{\boldsymbol{u}_{N}}^{(N+1)}\left(\Pi_{m_{1}}^{(M)}\left(\boldsymbol{v}_{N} ; \tilde{C}_{N}\right)\right),
$$

for all $A \subseteq \Lambda$. For $M=0$, it follows from (7.14) that

$$
\sum_{\boldsymbol{v}_{N}} J_{\boldsymbol{u}_{N}, \boldsymbol{v}_{N}} \Pi_{m_{1}}^{(0)}\left(\boldsymbol{v}_{N} ; \tilde{C}_{N}\right)=p_{c} \delta_{m_{\boldsymbol{u}_{N}}, m_{1}-1} .
$$

For $M \geqslant 1$, we use (3.27), (7.14) and (3.38) to rewrite the right-hand side of (8.28). This gives

$$
\left|\kappa_{m_{1}, n+1}^{(N)}(A)\right| \leqslant p_{c} \sum_{M=0}^{\infty} \sum_{y} \pi_{m_{1}-1}^{(N+M)}(y)
$$

The combination of (8.27), (8.30) and (6.9) gives

$$
\left|e_{n+1}^{(N)}(3)\right| \leqslant 2 p_{c}^{2} \sum_{M=0}^{\infty} \sum_{m_{1}=\lfloor(n+1) / 2\rfloor+1}^{n+1} \sum_{y} P_{m_{1}-1}^{(N+M)}(y) \theta_{n+1-m_{1}} .
$$

It now follows from Lemma 8.2 and (6.2) that if $\beta$ is sufficiently small, then

$$
\left|e_{n+1}^{(N)}(3)\right| \leqslant C C_{\theta} \sum_{M=0}^{\infty}(C \beta)^{(N+M) \vee 1}(n+1)^{-d / 2} \log n \leqslant C C_{\theta}(C \beta)^{N \vee 1}(n+1)^{-d / 2} \log n,
$$

as required.

\subsection{Bound on $e_{n+1}^{(N)}(5)$}

Proof of (8.8). The error term $e_{n+1}^{(N)}(5)$ is defined in (5.18) in terms of the quantities given in (5.8)-(5.10). Let $e_{n+1}^{(N, M, K)}(5)$ denote the contribution to $e_{n+1}^{(N)}(5)$ due to $\Pi_{m_{1}}^{(M)}\left(\boldsymbol{v}_{N} ; \tilde{C}_{N}\right)$ and $\chi_{n+1}^{(K)}\left(\bar{b} ; \tilde{C}_{N}^{b}\right)$ in (5.8), so that

$$
e_{n+1}^{(N)}(5) \leqslant \sum_{M, K=0}^{\infty}\left|e_{n+1}^{(N, M, K)}(5)\right| .
$$

Apart from the sum over $m_{1}$ and factor $\theta_{n+1-m_{1}}$ in (5.18), $e_{n+1}^{(N)}(5)$ has the same form as $\phi_{m_{1}, m_{2}}^{(N)}$ (defined in (5.17) and (5.13)), except that the factor $\chi_{n+1}$ in (5.8) for $e_{n+1}^{(N)}(5)$ is replaced by $\Pi_{m_{2}}$ in (5.13) for $\phi_{m_{1}, m_{2}}^{(N)}$. We will estimate $e_{n+1}^{(N, M, K)}(5)$ by determining the effect of this replacement. Let

$$
T_{m_{1}, m_{2}}^{(N, M, K)}\left(y_{1}, y_{2}\right)=R_{m_{1}, m_{2}}^{(N, M)}\left(y_{1}, y_{2}\right)+Q_{m_{1}, m_{2}}^{(N, M, K)}\left(y_{1}, y_{2}\right)
$$


and

$$
T_{m_{1}, m_{2}}^{(N)}=p_{c}^{2} \sum_{K, M=0}^{\infty} \sum_{y_{1}, y_{2}} T_{m_{1}-1, m_{2}-1}^{(N, M, K)}\left(y_{1}, y_{2}\right) .
$$

For $N \geqslant 0$, it follows from (7.16) and Propositions 7.6, 7.7 that

$$
\left|\phi_{m_{1}, m_{2}}^{(N)}\right| \leqslant T_{m_{1}, m_{2}}^{(N)} \leqslant C(C \beta)^{N} b_{m_{1}, m_{2}} .
$$

If the factor $\chi_{n+1}^{(K)}\left(\bar{b} ; \tilde{C}_{N}^{b}\right)$ appearing in (5.9), (5.10) were replaced by $\Pi_{m_{2}}^{(K)}\left(\bar{b} ; \tilde{C}_{N}^{b}\right)$, then we would have as upper bound

$$
\sum_{m_{1}=1}^{\lfloor(n+1) / 2\rfloor} T_{m_{1}, m_{2}}^{(N, M)} p_{c} \theta_{n+1-m_{1}} .
$$

The upper bound on $\chi_{n+1}^{(K)}\left(\bar{b} ; \tilde{C}_{N}^{b}\right)$ in Proposition 6.7 is given explicitly in terms of the diagrams used to bound $\Pi_{m_{2}}^{(K)}\left(\bar{b} ; \tilde{C}_{N}^{b}\right)$. The appropriate modification for $e_{n+1}^{(N)}(5)$ is thus

$$
\left|e_{n+1}^{(N)}(5)\right| \leqslant p_{c}^{2} \sum_{m_{1}=1}^{\lfloor(n+1) / 2\rfloor} \sum_{m_{2}=m_{1}}^{n+1}\left(T_{m_{1}, m_{2}}^{(N)}\left(\theta_{n+1}\right) \wedge T_{m_{1}, m_{2}}^{(N)}\right) \theta_{n+1-m_{1}} \theta_{n+1-m_{2}} .
$$

It then follows from (8.36) and Lemma 8.6 that

$$
\left|e_{n+1}^{(N)}(5)\right| \leqslant C C_{\theta}^{3}(C \beta)^{N}(n+1)^{-3}\left[\Delta_{n+1}^{\prime}+(n+1)^{(6-d) / 2} \log n\right] .
$$

When $N=0$, the only contribution without a positive power of $\beta$ arises when $m_{1}=m_{2}=1$ in (8.38), and this contribution is bounded above by $C C_{\theta}^{3}(n+1)^{-3}$. Therefore,

$$
\left|e_{n+1}^{(N)}(5)\right| \leqslant C C_{\theta}^{3}(n+1)^{-3}\left[\delta_{N, 0}+(C \beta)^{N \vee 1}\left[\Delta_{n+1}^{\prime}+(n+1)^{(6-d) / 2} \log n\right]\right],
$$

which completes the proof of (8.8).

\subsection{Bound on $e_{n+1}^{(N)}(2)$}

We prove a lemma before proving the bound (8.5) on $e_{n+1}^{(N)}(2)$. The lemma generalises (6.21). Note that the order in which constructions are applied can be important, since, e.g., $P^{(N)}\left(\boldsymbol{y} ; \ell^{\lambda}(\boldsymbol{a}), \theta_{n+1}\right)$ is not equal to $P^{(N)}\left(\boldsymbol{y} ; \theta_{n+1}, \ell^{\lambda}(\boldsymbol{a})\right)$.

Lemma 8.7. For $N, M \geqslant 0, \boldsymbol{y} \in \Lambda$, and $n \geqslant 0$,

$$
\sum_{\lambda} \sum_{\boldsymbol{a}} \sum_{\left(\boldsymbol{u}_{N}, \boldsymbol{v}_{N}\right)} J_{\boldsymbol{u}_{N}, \boldsymbol{v}_{N}} P^{(N)}\left(\boldsymbol{u}_{N} ; \ell^{\lambda}(\boldsymbol{a}), \theta_{n+1}\right) P^{(M)}\left(\boldsymbol{v}_{N}, \boldsymbol{y} ; \boldsymbol{a}, \theta_{n+1}\right) \leqslant P^{(N+M+1)}\left(\boldsymbol{y} ; \theta_{n+1}, \theta_{n+1}\right),
$$

where the sum over $\lambda$ is over the $N$-admissible lines for $P^{(N)}$.

Proof. Eq. (8.41) is similar to (6.21), but now with two extra Constructions $\theta_{n+1}$ performed. The equality in (6.21) is replaced by an upper bound in (8.41), since on the right-hand side there are more possibilities for the lines on which the two Constructions $\theta_{n+1}$ can be performed.

Proof of (8.5). The error term $e_{n+1}^{(N)}(2)$ is defined in (4.30) and (4.19). For $A=\tilde{C}_{N-1}$ or $A=\left\{\boldsymbol{v}_{N-1}\right\}$, we use

$$
I\left[\boldsymbol{v} \stackrel{A}{\rightarrow} n+1 \text { in } \tilde{C}_{N}\right] \leqslant I[v \rightarrow n+1]
$$

to obtain 


$$
\begin{aligned}
\left|e_{n+1}^{(N)}(2)\right| & \leqslant 2 \sum_{\left(\boldsymbol{u}_{N}, \boldsymbol{v}_{N}\right)} J_{\boldsymbol{u}_{N}, \boldsymbol{v}_{N}} M_{\boldsymbol{u}_{N}}^{(N+1)}\left(I\left[\boldsymbol{v}_{N-1} \rightarrow n+1\right]\left|\chi_{n+1}\left(\boldsymbol{v}_{N} ; \tilde{C}_{N}\right)\right|\right) \\
& \leqslant 2 \sum_{M=0}^{\infty} \sum_{\left(\boldsymbol{u}_{N}, \boldsymbol{v}_{N}\right)} J_{\boldsymbol{u}_{N}, \boldsymbol{v}_{N}} M_{\boldsymbol{u}_{N}}^{(N+1)}\left(I\left[\boldsymbol{v}_{N-1} \rightarrow n+1\right]\left|\chi_{n+1}^{(M)}\left(\boldsymbol{v}_{N} ; \tilde{C}_{N}\right)\right|\right) .
\end{aligned}
$$

We use Proposition 6.7 to bound $\left|\chi_{n+1}^{(M)}\left(\boldsymbol{v}_{N} ; \tilde{C}_{N}\right)\right|$, using the second alternative for the minimum in (6.28) when $k \geqslant\lfloor n / 2\rfloor+1$, and the first alternative in (6.28) when $k \leqslant\lfloor n / 2\rfloor$. Denote the contribution due to $k \geqslant\lfloor n / 2\rfloor+1$ by $e_{n+1}^{(N)}(2,1)$, and the contribution due to $k \leqslant\lfloor n / 2\rfloor$ by $e_{n+1}^{(N)}(2,2)$, so that

$$
\left|e_{n+1}^{(N)}(2)\right| \leqslant 2\left(e_{n+1}^{(N)}(2,1)+e_{n+1}^{(N)}(2,2)\right) .
$$

For $e_{n+1}^{(N)}(2,1)$, we use $(6.28)$ and (7.8) to obtain

$$
\begin{aligned}
e_{n+1}^{(N)}(2,1) & \leqslant \sum_{M=0}^{\infty} \sum_{\boldsymbol{a}} \sum_{\left(\boldsymbol{u}_{N}, \boldsymbol{v}_{N}\right)} J_{\boldsymbol{u}_{N}, \boldsymbol{v}_{N}} M_{\boldsymbol{u}_{N}}^{(N+1)}\left(I\left[\boldsymbol{a} \in \tilde{C}_{N}\right]\right) \sum_{k=\lfloor n / 2\rfloor+1}^{n+1} \sum_{y \in \mathbb{Z}^{d}} P^{(M)}\left(\boldsymbol{v}_{N},(y, k) ; \boldsymbol{a}\right) p_{c} \theta_{n-k} \\
& \leqslant \sum_{M=0}^{\infty} \sum_{k=\lfloor n / 2\rfloor+1}^{n+1} \sum_{y \in \mathbb{Z}^{d}} \sum_{\lambda} \sum_{\boldsymbol{a}} \sum_{\left(\boldsymbol{u}_{N}, \boldsymbol{v}_{N}\right)} J_{\boldsymbol{u}_{N}, \boldsymbol{v}_{N}} P^{(N)}\left(\boldsymbol{u}_{N} ; \ell^{\lambda}(\boldsymbol{a})\right) P^{(M)}\left(\boldsymbol{v}_{N},(y, k) ; \boldsymbol{a}\right) p_{c} \theta_{n-k},
\end{aligned}
$$

where we recall Remark 6.2. By (6.21), (6.2) and Lemma 8.2,

$$
\begin{aligned}
e_{n+1}^{(N)}(2,1) & \leqslant \sum_{M=0}^{\infty} \sum_{k=\lfloor n / 2\rfloor+1}^{n+1} \sum_{y \in \mathbb{Z}^{d}} P_{k}^{(N+M)}(y) p_{c} \theta_{n-k} \leqslant C C_{\theta} \sum_{M=0}^{\infty}(C \beta)^{(N+M) \vee 1}(n+1)^{-d / 2} \log n \\
& \leqslant C C_{\theta}(C \beta)^{N \vee 1}(n+1)^{-d / 2} \log n .
\end{aligned}
$$

For $e_{n+1}^{(N)}(2,2)$, we use the minor extension of (7.8) that, for $N \geqslant 0, \boldsymbol{a}, \boldsymbol{y} \in \Lambda$ and $n \geqslant 0$,

$$
M_{\boldsymbol{y}}^{(N+1)}\left(I\left[\boldsymbol{a} \in \tilde{C}_{N}, \boldsymbol{v}_{N-1} \rightarrow n+1\right]\right) \leqslant \sum_{\lambda} P^{(N)}\left(\boldsymbol{y} ; \ell^{\lambda}(\boldsymbol{a}), \theta_{n+1}\right) .
$$

By (8.47) and (6.28),

$$
\begin{aligned}
& e_{n+1}^{(N)}(2,2) \\
& \quad \leqslant \sum_{M=0}^{\infty} \sum_{k=0}^{\lfloor n / 2\rfloor} \sum_{\lambda} \sum_{\boldsymbol{a}} \sum_{\left(\boldsymbol{u}_{N}, \boldsymbol{v}_{N}\right)} J_{\boldsymbol{u}_{N}, \boldsymbol{v}_{N}} P^{(N)}\left(\boldsymbol{u}_{N} ; \ell^{\lambda}(\boldsymbol{a}), \theta_{n+1}\right) \sum_{y \in \mathbb{Z}^{d}} P^{(M)}\left(\boldsymbol{v}_{N},(y, k) ; \boldsymbol{a}, \theta_{n+1}\right) p_{c} \theta_{n-k} .
\end{aligned}
$$

It then follows from Lemma 8.7, Lemma 8.3 and (6.2) that

$$
\begin{aligned}
e_{n+1}^{(N)}(2,2) & \leqslant \sum_{M=0}^{\infty} \sum_{k=0}^{\lfloor n / 2\rfloor} \sum_{y \in \mathbb{Z}^{d}} P_{k}^{(N+M)}\left(y ; \theta_{n+1}, \theta_{n+1}\right) p_{c} \theta_{n-k} \\
& \leqslant C C_{\theta}^{3} \sum_{M=0}^{\infty}(C \beta)^{N+M}(n+1)^{-3} \Delta_{n+1}^{\prime} \leqslant C C_{\theta}^{3}(C \beta)^{N}(n+1)^{-3} \Delta_{n+1}^{\prime} .
\end{aligned}
$$

When $N=0$, the only contribution without a positive power of $\beta$ arises when $k=0$ in (8.49). This requires that $y=0$ and $M=0$, and contributes at most $C C_{\theta}^{3}(n+1)^{-3}$. With this observation, we can improve (8.49) to

$$
e_{n+1}^{(N)}(2,2) \leqslant C C_{\theta}^{3}(n+1)^{-3}\left[\delta_{N, 0}+(C \beta)^{N \vee 1} \Delta_{n+1}^{\prime}\right] .
$$

Substitution of (8.46) and (8.50) into (8.44) then gives (8.5). 


\subsection{Bound on $e_{n+1}^{(N)}(4)$}

In this section, we prove the bound (8.7) on the error term $e_{n+1}^{(N)}(4)$. By (5.18),

$$
e_{n+1}^{(N)}(4) \leqslant \sum_{m_{1}=1}^{\lfloor(n+1) / 2\rfloor}\left[\left|d_{m_{1}, n+1}^{(N)}\left(4 ;\left\{\boldsymbol{v}_{N-1}\right\}\right)\right|+\frac{1}{2}\left|d_{m_{1}, n+1}^{(N)}\left(4 ; \tilde{C}_{N-1}\right)\right|\right] \theta_{n+1-m_{1}} .
$$

We denote the contribution due to $d_{m_{1}, n+1}^{(N)}\left(4 ;\left\{\boldsymbol{v}_{N-1}\right\}\right)$ by $e_{n+1}^{(N)}(4,1)$ and the contribution due to $d_{m_{1}, n+1}^{(N)}\left(4 ; \tilde{C}_{N-1}\right)$ by $e_{n+1}^{(N)}(4,2)$. We prove bounds on $e_{n+1}^{(N)}(4,1)$ in Sections 8.6.1, 8.6.2, and on $e_{n+1}^{(N)}(4,2)$ in Section 8.6.3. The proof of (8.7) is completed at the end of Section 8.6.3.

\subsubsection{Preliminaries for $e_{n+1}^{(N)}(4,1)$}

Our bound on $e_{n+1}^{(N)}(4,1)$ uses the following two lemmas.

Lemma 8.8. Let $\boldsymbol{v}_{N-1}, \boldsymbol{y} \in \Lambda$ and let $m_{1}<m_{\boldsymbol{y}} \leqslant n$. Let $F=F(\boldsymbol{y})$ be an event that depends only on bonds with time variables at most $m_{y}$. Then

$$
\begin{aligned}
& \mathbb{E}_{N}\left(I[F(\boldsymbol{y})] I\left[\left(\boldsymbol{v}_{N-1} \rightarrow n\right) \circ(\boldsymbol{y} \rightarrow n)\right] \Pi_{m_{1}}^{(M)}\left(\boldsymbol{v}_{N} ; \tilde{C}_{N}\right)\right) \\
& \quad \leqslant \mathbb{E}_{N}\left(I[F(\boldsymbol{y})] I\left[\left(\boldsymbol{v}_{N-1} \rightarrow n\right)\right] \Pi_{m_{1}}^{(M)}\left(\boldsymbol{v}_{N} ; \tilde{C}_{N}\right)\right) \theta_{n}(\boldsymbol{y}) .
\end{aligned}
$$

Proof. Let $C_{\boldsymbol{y}}(\boldsymbol{v})=\left\{\boldsymbol{x} \in C(\boldsymbol{v}): m_{\boldsymbol{x}}=m_{\boldsymbol{y}}\right\}$. By the Markov property,

$$
\begin{aligned}
& \mathbb{E}_{N}\left(I[F(\boldsymbol{y})] I\left[\left(\boldsymbol{v}_{N-1} \rightarrow n\right) \circ(\boldsymbol{y} \rightarrow n)\right] \Pi_{m_{1}}^{(M)}\left(\boldsymbol{v}_{N} ; \tilde{C}_{N}\right)\right) \\
& \left.\quad=\sum_{C \neq \varnothing} \mathbb{E}_{N}\left(I[F(\boldsymbol{y})] I\left[C_{\boldsymbol{y}}\left(\boldsymbol{v}_{N-1}\right)=C\right]\right) \Pi_{m_{1}}^{(M)}\left(\boldsymbol{v}_{N} ; \tilde{C}_{N}\right)\right) \mathbb{P}_{N}((C \rightarrow n) \circ(\boldsymbol{y} \rightarrow n)),
\end{aligned}
$$

since $\Pi_{m_{1}}^{(M)}\left(\boldsymbol{v}_{N} ; \tilde{C}_{N}\right)$ is independent of bonds with time variables exceeding $m_{\boldsymbol{y}}$ because $m_{\boldsymbol{y}} \geqslant m_{1}-1$. By the BK inequality,

$$
\mathbb{P}_{N}((C \rightarrow n) \circ(\boldsymbol{y} \rightarrow n)) \leqslant \mathbb{P}_{N}(C \rightarrow n) \theta_{n}(\boldsymbol{y}) .
$$

The inequality (8.52) can then be concluded from substitution of (8.54) into (8.53) followed by summation over $C$.

For $m_{v} \leqslant m \leqslant n$, we define

$$
V_{m, n}(\boldsymbol{v})=\bigcup_{z: m_{z} \leqslant m}\{\boldsymbol{v} \rightarrow z \Longrightarrow n\}
$$

For later use, we note that it follows from (8.55) that

$$
V_{m, n}(\boldsymbol{v})=\bigcup_{\boldsymbol{y}: m_{\boldsymbol{y}}=m}\{\boldsymbol{v} \rightarrow \boldsymbol{y}\} \cap\{(\boldsymbol{y} \rightarrow n) \circ(\boldsymbol{v} \rightarrow n)\} .
$$

The following lemma is reminiscent of Lemma 7.15.

Lemma 8.9. For $\boldsymbol{v}_{N-1} \in \Lambda$ and $m, n \geqslant 1$ such that $m_{\boldsymbol{v}_{N-1}}<m \leqslant n$,

$$
H_{m, n}\left(\boldsymbol{v}_{N-1} ;\left\{\boldsymbol{v}_{N-1}\right\}\right) \subseteq V_{m-1, n}\left(\boldsymbol{v}_{N-1}\right) .
$$

Proof. When $H_{m, n}\left(\boldsymbol{v}_{N-1} ;\left\{\boldsymbol{v}_{N-1}\right\}\right)$ occurs, there is no $m$-cutting bond for $\boldsymbol{v}_{N-1} \rightarrow n$. Let $b$ be the last pivotal bond for $\boldsymbol{v}_{N-1} \rightarrow n$, if it exists. If such a bond does not exist, then $\boldsymbol{v}_{N-1} \Rightarrow n$, which is a subset of $V_{m-1, n}\left(\boldsymbol{v}_{N-1}\right)$. On the other hand, if there is such a bond $b$, then $\bar{b} \Rightarrow n$, and therefore $m_{\bar{b}} \leqslant m-1$, since otherwise $b$ would be the $m$-cutting bond for $\boldsymbol{v}_{N-1} \rightarrow n$. Thus, $V_{m-1, n}\left(\boldsymbol{v}_{N-1}\right)$ occurs. 
We also use the minor modification of (8.47) that for every $\boldsymbol{a}, \boldsymbol{y} \in \Lambda, N \geqslant 0$, and $n \geqslant 0$,

$$
M_{\boldsymbol{u}_{N}}^{(N+1)}\left(I\left[\left(\boldsymbol{v}_{N-1} \rightarrow \boldsymbol{a}\right) \cap\left(\boldsymbol{v}_{N-1} \rightarrow \boldsymbol{y}\right) \cap\left(\boldsymbol{v}_{N-1} \rightarrow n\right)\right]\right) \leqslant \sum_{\lambda} P^{(N)}\left(\boldsymbol{u}_{N} ; \ell^{\lambda}(\boldsymbol{a}), \ell(\boldsymbol{y}), \theta_{n}\right),
$$

where the sum over $\lambda$ again runs over the $N$-admissible lines for $P^{(N)}\left(\boldsymbol{u}_{N}\right)$.

\subsubsection{Bound on $e_{n+1}^{(N)}(4,1)$}

In this section, we prove that

$$
\left|e_{n+1}^{(N)}(4,1)\right| \leqslant C_{\theta}^{3}(C \beta)^{N}(n+1)^{-3} \text {. }
$$

The definitions (5.9), (5.10) and (5.7) imply that

$$
d_{m_{1}, n+1}^{(0)}(4 ; A)=\sum_{\left(\boldsymbol{u}_{0}, \boldsymbol{v}_{0}\right)} J_{\boldsymbol{u}_{0}, \boldsymbol{v}_{0}} \widetilde{\mathbb{E}}_{N}\left(I\left[\left\{\mathbf{0} \Rightarrow \boldsymbol{u}_{0}\right\} \cap H_{m_{1}, n}(\mathbf{0} ; A)\right] \Pi_{m_{1}}\left(\boldsymbol{v}_{0} ; \tilde{C}_{0}\right)\right),
$$

while, for $N \geqslant 1$,

$$
\begin{aligned}
d_{m_{1}, n+1}^{(N)}(4 ; A)= & \sum_{\left(\boldsymbol{u}_{N-1}, \boldsymbol{v}_{N-1}\right)} J_{\boldsymbol{u}_{N-1}, \boldsymbol{v}_{N-1}} \sum_{\left(\boldsymbol{u}_{N}, \boldsymbol{v}_{N}\right)} J_{\boldsymbol{u}_{N}, \boldsymbol{v}_{N}} \\
& \times M_{\boldsymbol{u}_{N-1}}^{(N)}\left(\widetilde{\mathbb{E}}_{N}\left(I\left[E^{\prime}\left(\boldsymbol{v}_{N-1}, \boldsymbol{u}_{N} ; \tilde{C}_{N-1}\right) \cap H_{m_{1}, n}\left(\boldsymbol{v}_{N-1} ; A\right)\right] \Pi_{m_{1}}\left(\boldsymbol{v}_{N} ; \tilde{C}_{N}\right)\right)\right) .
\end{aligned}
$$

We denote by $d_{m_{1}, n+1}^{(N, M)}(4 ; A)$ the contribution due to $\Pi_{m_{1}}^{(M)}\left(\boldsymbol{v}_{N} ; \tilde{C}_{N}\right)$ in $(8.60),(8.61)$, so that

$$
\left|d_{m_{1}, n+1}^{(N)}(4 ; A)\right| \leqslant \sum_{M=0}^{\infty} d_{m_{1}, n+1}^{(N, M)}(4 ; A) .
$$

Using Lemma 8.9, (8.56) and Lemmas 7.16 and 8.8, we obtain

$$
\begin{aligned}
& \widetilde{\mathbb{E}}_{N}\left(I\left[E^{\prime}\left(\boldsymbol{v}_{N-1}, \boldsymbol{u}_{N} ; \tilde{C}_{N-1}\right) \cap H_{m_{1}, n}\left(\boldsymbol{v}_{N-1} ; A\right)\right] \Pi_{m_{1}}^{(M)}\left(\boldsymbol{v}_{N} ; \tilde{C}_{N}\right)\right) \\
& \quad \leqslant \sum_{\boldsymbol{y}_{2}: m_{\boldsymbol{y}_{2}=m_{1}-1}} \widetilde{\mathbb{E}}_{N}\left(I\left[E^{\prime}\left(\boldsymbol{v}_{N-1}, \boldsymbol{u}_{N} ; \tilde{C}_{N-1}\right)\right] I\left[\left(\boldsymbol{v}_{N-1} \rightarrow \boldsymbol{y}_{2}\right)\right] I\left[\left(\boldsymbol{v}_{N-1} \rightarrow n\right) \circ\left(\boldsymbol{y}_{2} \rightarrow n\right)\right] \Pi_{m_{1}}^{(M)}\left(\boldsymbol{v}_{N} ; \tilde{C}_{N}\right)\right) \\
& \quad \leqslant \sum_{\boldsymbol{y}_{2}: m_{\boldsymbol{y}_{2}}=m_{1}-1} \mathbb{E}_{N}\left(I\left[E^{\prime}\left(\boldsymbol{v}_{N-1}, \boldsymbol{u}_{N} ; \tilde{C}_{N-1}\right)\right] I\left[\left(\boldsymbol{v}_{N-1} \rightarrow \boldsymbol{y}_{2}\right) \cap\left(\boldsymbol{v}_{N-1} \rightarrow n\right)\right] \Pi_{m_{1}}^{(M)}\left(\boldsymbol{v}_{N} ; \tilde{C}_{N}\right)\right) \theta_{n}\left(\boldsymbol{y}_{2}\right) .
\end{aligned}
$$

Using (7.14) and (6.9), it follows that, for $M \geqslant 1$,

$$
\begin{aligned}
& \mathbb{E}_{N}\left(I\left[E^{\prime}\left(\boldsymbol{v}_{N-1}, \boldsymbol{u}_{N} ; \tilde{C}_{N-1}\right) \cap H_{m_{1}, n}\left(\boldsymbol{v}_{N-1} ; A\right)\right] \Pi_{m_{1}}^{(M)}\left(\boldsymbol{v}_{N} ; \tilde{C}_{N}\right)\right) \\
& \leqslant \sum_{y_{2}} \sum_{\boldsymbol{a}} \mathbb{E}_{N}\left(I\left[E^{\prime}\left(\boldsymbol{v}_{N-1}, \boldsymbol{u}_{N} ; \tilde{C}_{N-1}\right) \cap\left\{\boldsymbol{v}_{N-1} \rightarrow \boldsymbol{a}\right\} \cap\left\{\boldsymbol{v}_{N-1} \rightarrow\left(y_{2}, m_{1}-1\right)\right\} \cap\left\{\boldsymbol{v}_{N-1} \rightarrow n\right\}\right]\right) \\
& \quad \times \sum_{y_{1}} p_{c} P^{(M-1)}\left(\boldsymbol{v}_{N},\left(y_{1}, m_{1}-1\right) ; \boldsymbol{a}\right) \theta_{n}\left(\boldsymbol{y}_{2}\right) .
\end{aligned}
$$

When $M=0$, a similar bound holds with both the sum over $\boldsymbol{a}$ and the indicator that $\boldsymbol{v}_{N-1} \rightarrow \boldsymbol{a}$ removed, and with $p_{c} P^{(M-1)}\left(\boldsymbol{v}_{N},\left(y_{1}, m_{1}-1\right) ; \boldsymbol{a}\right)$ replaced by $\delta_{m_{1}, m_{v_{N}}}$. This causes only minor modifications, and in the remainder of the proof of (8.59) we consider only the case $M \geqslant 1$ explicitly.

Now we specialise to $A=\left\{\boldsymbol{v}_{N-1}\right\}$, as in the definition of $e_{n+1}^{(N)}(4,1)$. For $N=0$, we use the convention (4.15) and apply (8.64) and (8.58) to obtain

$$
\begin{aligned}
d_{m_{1}, n+1}^{(0, M)}(4 ;\{\boldsymbol{0}\}) \leqslant & \sum_{y_{2}} \sum_{\lambda} \sum_{\left(\boldsymbol{u}_{0}, \boldsymbol{v}_{0}\right)} J_{\boldsymbol{u}_{0}, \boldsymbol{v}_{0}} \sum_{\boldsymbol{a}} P^{(0)}\left(\boldsymbol{u}_{0} ; \ell^{\lambda}(\boldsymbol{a}), \ell\left(y_{2}, m_{1}-1\right), \theta_{n+1}\right) \\
& \times \sum_{y_{1}} p_{c} P^{(M-1)}\left(\boldsymbol{v}_{0},\left(y_{1}, m_{1}-1\right) ; \boldsymbol{a}\right) \theta_{n+1}\left(y_{2}, m_{1}-1\right) .
\end{aligned}
$$


For $N \geqslant 1$, we substitute (8.64) into (8.61), use the recursion (3.35) in the form

$$
\begin{aligned}
& \sum_{\left(\boldsymbol{u}_{N-1}, \boldsymbol{v}_{N-1}\right)} J_{\boldsymbol{u}_{N-1}, \boldsymbol{v}_{N-1}} M_{\boldsymbol{u}_{N-1}}^{(N)}\left(\mathbb{E}_{N}\left(I\left[E^{\prime}\left(\boldsymbol{v}_{N-1}, \boldsymbol{u}_{N} ; \tilde{C}_{N-1}\right) \cap\left\{\left(\boldsymbol{v}_{N-1} \rightarrow \boldsymbol{a}\right) \cap\left(\boldsymbol{v}_{N-1} \rightarrow \boldsymbol{y}_{2}\right) \cap\left(\boldsymbol{v}_{N-1} \rightarrow n\right)\right\}\right]\right)\right) \\
& =M_{\boldsymbol{u}_{N}}^{(N+1)}\left(I\left[\left(\boldsymbol{v}_{N-1} \rightarrow \boldsymbol{a}\right) \cap\left(\boldsymbol{v}_{N-1} \rightarrow \boldsymbol{y}_{2}\right) \cap\left(\boldsymbol{v}_{N-1} \rightarrow n\right)\right]\right)
\end{aligned}
$$

and apply (8.58), to obtain

$$
\begin{aligned}
d_{m_{1}, n+1}^{(N, M)}\left(4 ;\left\{\boldsymbol{v}_{N-1}\right\}\right) \leqslant & \sum_{y_{2}} \sum_{\lambda} \sum_{\left(\boldsymbol{u}_{N}, \boldsymbol{v}_{N}\right)} J_{\boldsymbol{u}_{N}, \boldsymbol{v}_{N}} \sum_{\boldsymbol{a}} P^{(N)}\left(\boldsymbol{u}_{N} ; \ell^{\lambda}(\boldsymbol{a}), \ell\left(y_{2}, m_{1}-1\right), \theta_{n+1}\right) \\
& \times \sum_{y_{1}} p_{c} P^{(M-1)}\left(\boldsymbol{v}_{N},\left(y_{1}, m_{1}-1\right) ; \boldsymbol{a}\right) \theta_{n+1}\left(y_{2}, m_{1}-1\right) .
\end{aligned}
$$

Thus, the bounds for $N=0$ and $N \geqslant 1$ in (8.65) and (8.67) agree, and we can henceforth treat all $N \geqslant 0$ simultaneously.

By (8.67) and a slight generalisation of (6.21) (similar to Lemma 8.7),

$$
d_{m_{1}, n+1}^{(N, M)}\left(4 ;\left\{\boldsymbol{v}_{N-1}\right\}\right) \leqslant p_{c} \sum_{y_{1}, y_{2}} P_{m_{1}-1}^{(N+M)}\left(y_{1} ; \ell\left(y_{2}, m_{1}-1\right), \theta_{n+1}\right) \theta_{n+1}\left(y_{2}, m_{1}-1\right) .
$$

Let

$$
B_{m}^{(N+M)}=\sum_{y_{1}, y_{2}} P_{m}^{(N+M)}\left(y_{1} ; \ell\left(y_{2}, m\right)\right) .
$$

By the definition of $e_{n+1}^{(N)}(4,1)$ below (8.51), we therefore obtain

$$
\left|e_{n+1}^{(N)}(4,1)\right| \leqslant p_{c} \sum_{M=0}^{\infty} \sum_{m_{1}=1}^{\lfloor(n+1) / 2\rfloor} B_{m_{1}-1}^{(N+M)}\left(\theta_{n+1}\right) \theta_{n-m_{1}}^{2} .
$$

By (6.2) and Lemma 7.8(b),

$$
B_{m}^{(N+M)} \leqslant(C \beta)^{N+M}(m+1)^{-(d-2) / 2},
$$

so it follows from (8.70) and Lemma 8.5 that

$$
\left|e_{n+1}^{(N)}(4,1)\right| \leqslant \sum_{M=0}^{\infty} C_{\theta}^{3}(C \beta)^{N+M}(n+1)^{-3}=C_{\theta}^{3}(C \beta)^{N}(n+1)^{-3} .
$$

This proves (8.59).

\subsubsection{Bound on $e_{n+1}^{(N)}(4,2)$}

In this section, we prove that

$$
\left|e_{n+1}^{(N)}(4,2)\right| \leqslant C C_{\theta}^{3}(n+1)^{-3} \delta_{N, 0}+C_{\theta}^{3}(C \beta)^{N \vee 1}\left[(n+1)^{-d / 2} \log n+(n+1)^{-3} \Delta_{n+1}^{\prime}\right] .
$$

Similarly to (7.86)-(7.88), we define

$$
\begin{aligned}
& G_{m, n}^{(1)}\left(\boldsymbol{v}_{N-1}\right)=H_{m, n}\left(\boldsymbol{v}_{N-1} ; \tilde{C}_{N-1}\right) \cap V_{m-1, n}\left(\boldsymbol{v}_{N-1}\right), \\
& G_{m, n}^{(2)}\left(\boldsymbol{v}_{N-1}\right)=H_{m, n}\left(\boldsymbol{v}_{N-1} ; \tilde{C}_{N-1}\right) \cap V_{m-1, n}\left(\boldsymbol{v}_{N-1}\right)^{c},
\end{aligned}
$$

so that

$$
H_{m, n}\left(\boldsymbol{v}_{N-1} ; \tilde{C}_{N-1}\right)=G_{m, n}^{(1)}\left(\boldsymbol{v}_{N-1}\right) \dot{\cup} G_{m, n}^{(2)}\left(\boldsymbol{v}_{N-1}\right) .
$$

For the contribution due to $G_{n, 1}^{(1)}\left(\boldsymbol{v}_{N-1}, \boldsymbol{u}_{N}\right)$, we recall the argument beginning with (8.63) to see that the inclusion $G_{m, n}^{(1)}\left(\boldsymbol{v}_{N-1}\right) \subseteq V_{m-1, n}\left(\boldsymbol{v}_{N-1}\right)$ implies that this term obeys the same bound as $\left|e_{n+1}^{(N)}(4,1)\right|$. Thus, it remains to bound 
the contribution due to $G_{m, n}^{(2)}\left(\boldsymbol{v}_{N-1}\right)$. Note that, by (4.15), $\tilde{C}_{-1}=\left\{\boldsymbol{v}_{-1}\right\}=\{\boldsymbol{0}\}$, so that, by Lemma 8.9 and (8.75), $G_{m, n}^{(2)}\left(\boldsymbol{v}_{-1}\right)=\varnothing$. Thus, we consider only $N \geqslant 1$ in the following.

Let $e_{n+1}^{(N)}(4,3)$ denote the contribution to $e_{n+1}^{(N)}(4,2)$ (defined below (8.51)) due to $G_{m, n+1}^{(2)}\left(\boldsymbol{v}_{N-1}\right)$, i.e.,

$$
e_{n+1}^{(N)}(4,3)=\frac{1}{2} \sum_{m_{1}=1}^{\lfloor(n+1) / 2\rfloor} d_{m_{1}, n+1}^{(N)}\left(4 ; \tilde{C}_{N-1}, 3\right) p_{c} \theta_{n+1-m_{1}},
$$

where, as in (8.61), for $N \geqslant 1$,

$$
\begin{aligned}
& d_{m_{1}, n+1}^{(N)}\left(4 ; \tilde{C}_{N-1}, 3\right)=\sum_{\left(\boldsymbol{u}_{N-1}, \boldsymbol{v}_{N-1}\right)} J_{\boldsymbol{u}_{N-1}, \boldsymbol{v}_{N-1}} \sum_{\left(\boldsymbol{u}_{N}, \boldsymbol{v}_{N}\right)} J_{\boldsymbol{u}_{N}, \boldsymbol{v}_{N}} \\
& \times M_{\boldsymbol{u}_{N-1}}^{(N)}\left(\widetilde{\mathbb{E}}_{N}\left(I\left[E^{\prime}\left(\boldsymbol{v}_{N-1}, \boldsymbol{u}_{N} ; \tilde{C}_{N-1}\right) \cap G_{m_{1}, n}^{(2)}\left(\boldsymbol{v}_{N-1}\right)\right] \Pi_{m_{1}}\left(\boldsymbol{v}_{N} ; \tilde{C}_{N}\right)\right)\right) .
\end{aligned}
$$

It remains to estimate $e_{n+1}^{(N)}(4,3)$ for $N \geqslant 1$. The following analogue of Lemma 7.17 will be useful for this. Recall that the event $\mathcal{E}_{m}$ was defined in (7.48).

Lemma 8.10. For $\boldsymbol{v}_{N-1} \in \Lambda$ and $m, n \geqslant 1$,

$$
G_{m, n}^{(2)}\left(\boldsymbol{v}_{N-1}\right) \subseteq \bigcup_{\boldsymbol{y}_{2}}\left(\mathcal{E}_{m}\left(\boldsymbol{v}_{N-1}, \boldsymbol{y}_{2} ; \tilde{C}_{N-1}\right) \cap\left(\left(\boldsymbol{y}_{2} \rightarrow n\right) \circ\left(\boldsymbol{v}_{N-1} \rightarrow n\right)\right)\right) .
$$

Proof. Suppose that $G_{m, n}^{(2)}\left(\boldsymbol{v}_{N-1}\right)$ occurs. It cannot be the case that $\boldsymbol{v}_{N-1} \Rightarrow n$, since this contributes instead to $G_{m, n}^{(1)}\left(\boldsymbol{v}_{N-1}\right)$. Thus there is at least one occupied pivotal bond for $\boldsymbol{v}_{N-1} \rightarrow n$, and we denote the last such bond by $b$. Then $\left\{\boldsymbol{v}_{N-1} \rightarrow \bar{b} \Rightarrow n\right\}$ occurs. It must be the case that $m_{\bar{b}}>m-1$, since otherwise $G_{m, n}^{(1)}\left(\boldsymbol{v}_{N-1}\right)$ occurs. By the definition of $H_{m, n}\left(\boldsymbol{v}_{N-1} ; \tilde{C}_{N-1}\right)$ in (5.6), $b$ cannot be an $m$-cutting bond for $\boldsymbol{v}_{N-1} \stackrel{\tilde{C}_{N-1}}{\longrightarrow} n$, and hence $\left\{\boldsymbol{v}_{N-1} \stackrel{\tilde{C}_{N-1}}{\longrightarrow} \underline{b}\right\}^{c}$ occurs. Since $\boldsymbol{v}_{N-1} \stackrel{\tilde{C}_{N-1}}{\longrightarrow} n$ occurs by (5.6), we conclude that $\bar{b} \stackrel{\tilde{C}_{N-1}}{\longrightarrow} n$.

Fix $w$ such that $\underline{b} \rightarrow(w, n)$ (such a $w$ must exist since $\underline{b} \rightarrow n$ ). Let $b^{\prime}$ be the first occupied pivotal bond for $\underline{b} \rightarrow(w, n)$, if it exists, such that $\underline{b} \stackrel{\tilde{C}_{N-1}}{\longrightarrow} \underline{b}^{\prime}$. If $b^{\prime}$ does not exist, then $\mathcal{E}_{m}\left(\boldsymbol{v}_{N-1},(w, n) ; \tilde{C}_{N-1}\right)$ occurs, which is contained in the right-hand side of $(\overline{8} .79)$ with $\boldsymbol{y}_{2}=(w, n)$. On the other hand, if $b^{\prime}$ does exist, then, since $m_{\bar{b}}>m-1$, the event

$$
\left(\boldsymbol{v}_{N-1} \rightarrow \bar{b}\right) \cap E^{\prime}\left(\bar{b}, \underline{b}^{\prime} ; \tilde{C}_{N-1}\right) \cap\left(\left(\underline{b}^{\prime} \rightarrow n\right) \circ\left(\boldsymbol{v}_{N-1} \rightarrow n\right)\right)
$$

occurs, which implies that the event on the right-hand side of (8.79) occurs with $\boldsymbol{y}_{2}=\underline{b}^{\prime}$.

In view of Lemma 8.10 and (8.78), it is useful to estimate, for $N \geqslant 1$ and $M \geqslant 0$,

$$
\sum_{\boldsymbol{y}_{2}} \widetilde{\mathbb{E}}_{N}\left(I\left[E^{\prime}\left(\boldsymbol{v}_{N-1}, \boldsymbol{u}_{N} ; \tilde{C}_{N-1}\right) \cap \mathcal{E}_{m_{1}}\left(\boldsymbol{v}_{N-1}, \boldsymbol{y}_{2} ; \tilde{C}_{N-1}\right) \cap\left(\left(\boldsymbol{y}_{2} \rightarrow n\right) \circ\left(\boldsymbol{v}_{N-1} \rightarrow n\right)\right)\right] \Pi_{m_{1}}^{(M)}\left(\boldsymbol{v}_{N} ; \tilde{C}_{N}\right)\right) .
$$

By Lemmas 7.16 and 8.8,

$$
\begin{aligned}
& \widetilde{\mathbb{E}}_{N}\left(I\left[E_{N}^{\prime} \cap \mathcal{E}_{m_{1}}\left(\boldsymbol{v}_{N-1}, \boldsymbol{y}_{2} ; \tilde{C}_{N-1}\right)\right] I\left[\left(\boldsymbol{y}_{2} \rightarrow n\right) \circ\left(\boldsymbol{v}_{N-1} \rightarrow n\right)\right] \Pi_{m_{1}}^{(M)}\left(\boldsymbol{v}_{N} ; \tilde{C}_{N}\right)\right) \\
& \quad \leqslant \mathbb{E}_{N}\left(I\left[E_{N}^{\prime} \cap \mathcal{E}_{m_{1}}\left(\boldsymbol{v}_{N-1}, \boldsymbol{y}_{2} ; \tilde{C}_{N-1}\right) \cap\left(\boldsymbol{v}_{N-1} \rightarrow n\right)\right] \Pi_{m_{1}}^{(M)}\left(\boldsymbol{v}_{N} ; \tilde{C}_{N}\right)\right) \theta_{n}\left(\boldsymbol{y}_{2}\right) .
\end{aligned}
$$

The recursion relation (3.38) implies that

$$
\begin{aligned}
& \sum_{\left(\boldsymbol{u}_{N-1}, \boldsymbol{v}_{N-1}\right)} J_{\boldsymbol{u}_{N-1}, \boldsymbol{v}_{N-1}} M_{\boldsymbol{u}_{N-1}}^{(N)}\left(\mathbb{E}_{N}\left(I\left[E_{N}^{\prime} \cap \mathcal{E}_{m_{1}}\left(\boldsymbol{v}_{N-1}, \boldsymbol{y}_{2} ; \tilde{C}_{N-1}\right) \cap\left(\boldsymbol{v}_{N-1} \rightarrow n\right)\right] \Pi_{m_{1}}^{(M)}\left(\boldsymbol{v}_{N} ; \tilde{C}_{N}\right)\right)\right) \\
& =M_{\boldsymbol{u}_{N}}^{(N+1)}\left(I\left[\mathcal{E}_{m_{1}}\left(\boldsymbol{v}_{N-1}, \boldsymbol{y}_{2} ; \tilde{C}_{N-1}\right) \cap\left(\boldsymbol{v}_{N-1} \rightarrow n\right)\right] \Pi_{m_{1}}^{(M)}\left(\boldsymbol{v}_{N} ; \tilde{C}_{N}\right)\right) .
\end{aligned}
$$

Let

$$
Q_{m_{1}, m_{2}}^{(N+M)}=\sum_{y_{1}, y_{2}} Q^{(N+M)}\left(\left(y_{1}, m_{1}\right),\left(y_{2}, m_{2}\right)\right)
$$


If we simply ignore the indicator that $\boldsymbol{v}_{N-1} \rightarrow n$, then it follows from Proposition 7.11 that

$$
\begin{aligned}
& \sum_{\left(\boldsymbol{u}_{N}, \boldsymbol{v}_{N}\right)} J_{\boldsymbol{u}_{N}, \boldsymbol{v}_{N}} M_{\boldsymbol{u}_{N}}^{(N+1)}\left(I\left[\mathcal{E}_{m_{1}}\left(\boldsymbol{v}_{N-1}, \boldsymbol{y} ; \tilde{C}_{N-1}\right)\right] I\left[\left(\boldsymbol{v}_{N-1} \rightarrow n\right)\right] \Pi_{m_{1}}^{(M)}\left(\boldsymbol{v}_{N} ; \tilde{C}_{N}\right)\right) \\
& \quad \leqslant p_{c} \sum_{y_{1}} Q^{(N+M)}\left(\left(y_{1}, m_{1}-1\right), \boldsymbol{y}\right) .
\end{aligned}
$$

On the other hand, it is a minor extension of Proposition 7.11 that the extra connection to $n$ can be accounted for by an application of Construction $\theta_{n}$, so that the upper bound of (8.85) can alternately be replaced by the upper bound $p_{c} \sum_{y_{1}} Q^{(N+M)}\left(\left(y_{1}, m_{1}-1\right), \boldsymbol{y} ; \theta_{n}\right)$. Therefore, replacing $n$ by $n+1$,

$$
\left|e_{n+1}^{(N)}(4,3)\right| \leqslant \frac{1}{2} p_{c} \sum_{M=0}^{\infty} \sum_{m_{1}=1}^{\lfloor(n+1) / 2\rfloor} \sum_{m_{2}=m_{1}}^{n}\left(Q_{m_{1}-1, m_{2}-1}^{(N+M)}\left(\theta_{n+1}\right) \bigwedge Q_{m_{1}-1, m_{2}-1}^{(N+M)}\right) \theta_{n+1-m_{1}} \theta_{n+1-m_{2}} .
$$

By (7.41),

$$
Q_{m_{1}-1, m_{2}-1}^{(N+M)} \leqslant(C \beta)^{N+M} b_{m_{1}-1, m_{2}-1} \leqslant C(C \beta)^{N+M} b_{m_{1}, m_{2}} .
$$

Finally, by Lemma 8.6, for $N \geqslant 1$ we have

$$
\left|e_{n+1}^{(N)}(4,3)\right| \leqslant C_{\theta}^{3}(C \beta)^{N}\left[(n+1)^{-d / 2} \log n+(n+1)^{-3} \Delta_{n+1}^{\prime}\right] .
$$

The combination of (8.59) and (8.88) then gives the bound (8.73) on $e_{n+1}^{(N)}(4,2)$.

Proof of (8.7). This immediately follows from summation of (8.59) and (8.73).

\subsection{Bound on $e_{n+1}^{(N)}(1)$}

In this section, we prove the bound (8.4) on the error term $e_{n+1}^{(N)}(1)$, which is defined in (4.22)-(4.24). Recall (4.35)(4.37). For $j=1,2,3$, let

$$
e_{n+1}^{(0)}(1, j)=e_{n+1}^{(0)}(\mathbf{0} ;\{\mathbf{0}\} ; j),
$$

and, for $N \geqslant 1$,

$$
e_{n}^{(N)}(1, j)=\sum_{\left(\boldsymbol{u}_{N-1}, \boldsymbol{v}_{N-1}\right)} J_{\boldsymbol{u}_{N-1}, \boldsymbol{v}_{N-1}} M_{\boldsymbol{u}_{N-1}}^{(N)}\left(e_{n}^{(0)}\left(\boldsymbol{v}_{N-1} ; \tilde{C}_{N-1} ; j\right)\right) .
$$

It follows from (4.34) and (4.23), (4.24) that

$$
e_{n+1}^{(N)}(1)=e_{n+1}^{(N)}(1,1)+e_{n+1}^{(N)}(1,2)+e_{n+1}^{(N)}(1,3)
$$

We estimate $e_{n+1}^{(N)}(1,1)$ and $e_{n+1}^{(N)}(1,2)$ in Sections 8.7.1 and 8.7.2. The more delicate $e_{n}^{(N)}(1,3)$ is estimated in Section 8.7.3. The proof of (8.4) is given at the beginning of Section 8.7.3.

\subsubsection{Bound on $e_{n+1}^{(N)}(1,1)$}

The error term $e_{n+1}^{(N)}(1,1)$ is non-negative by definition. For $N=0$,

$$
\begin{aligned}
e_{n+1}^{(0)}(1,1) & \leqslant \mathbb{P}\left(F_{n}^{\prime}(\mathbf{0} ;\{\mathbf{0}\}) \cap\left\{\left|\mathcal{P}_{\{\mathbf{0}\}}\right| \leqslant 1\right\}\right) \leqslant \sum_{x} \mathbb{P}\left(E^{\prime}(\mathbf{0},(x, n+1) ;\{\mathbf{0}\})\right) \\
& =\sum_{x} \pi_{n+1}^{(0)}(x) \leqslant \sum_{x} P_{n+1}^{(0)}(x) \leqslant C \beta(n+1)^{-d / 2},
\end{aligned}
$$

by (4.38), Proposition 4.6, (6.9) and (6.2). For $N \geqslant 1$, 


$$
\begin{aligned}
e_{n+1}^{(N)}(1,1) & \leqslant \sum_{\left(\boldsymbol{u}_{N-1}, \boldsymbol{v}_{N-1}\right)} J_{\boldsymbol{u}_{N-1}, \boldsymbol{v}_{N-1}} M_{\boldsymbol{u}_{N-1}}^{(N)}\left(\sum_{x} \mathbb{P}\left(E^{\prime}\left(\boldsymbol{v}_{N-1},(x, n+1) ; \tilde{C}_{N-1}\right)\right)\right) \\
& =M_{(x, n+1)}^{(N+1)}(1)=\sum_{x} \pi_{n+1}^{(N)}(x) \leqslant \sum_{x} P_{n+1}^{(N)}(x) \leqslant(C \beta)^{N}(n+1)^{-d / 2},
\end{aligned}
$$

by (8.90), Proposition 4.6, (6.9) and (6.2).

\subsubsection{Bound on $e_{n+1}^{(N)}(1,2)$}

For $N=0$, we use (4.39) to see that

$$
\left|e_{n+1}^{(0)}(1,2)\right| \leqslant \sum_{\boldsymbol{y}: m_{\boldsymbol{y}} \geqslant\lfloor n / 2\rfloor+1} \mathbb{P}\left(E^{\prime}(\mathbf{0}, \boldsymbol{y} ;\{\mathbf{0}\})\right) \theta_{n+1}(\boldsymbol{y}) .
$$

Therefore,

$$
\left|e_{n+1}^{(0)}(1,2)\right| \leqslant \sum_{m=\lfloor n / 2\rfloor+1}^{n+1} \sum_{y} \pi_{m}^{(0)}(y) \theta_{n+1-m} \leqslant \sum_{m=\lfloor n / 2\rfloor+1}^{n+1} \sum_{y} P_{m}^{(0)}(y) \theta_{n+1-m} \leqslant C \beta(n+1)^{-d / 2} \log n,
$$

by (6.9), (6.2), and Lemma 8.2. For $N \geqslant 1$,

$$
\begin{aligned}
\left|e_{n+1}^{(N)}(1,2)\right| \leqslant & \sum_{\left(\boldsymbol{u}_{N-1}, \boldsymbol{v}_{N-1}\right)} J_{\boldsymbol{u}_{N-1}, \boldsymbol{v}_{N-1}} M_{\boldsymbol{u}_{N-1}}^{(N)}\left(\sum_{\boldsymbol{y}: m_{\boldsymbol{y}} \geqslant\lfloor n / 2\rfloor+1} \mathbb{P}\left(E^{\prime}\left(\boldsymbol{v}_{N-1}, \boldsymbol{y} ; \tilde{C}_{N-1}\right)\right)\right) \theta_{n+1}(\boldsymbol{y}) \\
& =\sum_{\boldsymbol{y}: m_{\boldsymbol{y}} \geqslant\lfloor n / 2\rfloor+1} M_{\boldsymbol{y}}^{(N+1)}(1) \theta_{n+1}(\boldsymbol{y})=\sum_{m=\lfloor n / 2\rfloor+1}^{n+1} \sum_{y} \pi_{m}^{(N)}(y) \theta_{n+1-m} \\
& \leqslant \sum_{m=\lfloor n / 2\rfloor+1}^{n+1} \sum_{y} P_{m}^{(N)}(y) \theta_{n+1-m} \leqslant(C \beta)^{N}(n+1)^{-d / 2} \log n,
\end{aligned}
$$

by (8.90), (4.39), (6.9), (6.2) and Lemma 8.2.

\subsubsection{Bound on $e_{n+1}^{(N)}(1,3)$}

In this section, we prove that, for $N \geqslant 1$,

$$
\begin{aligned}
& \left|e_{n+1}^{(0)}(1,3)\right| \leqslant C C_{\theta}^{3}\left[(n+1)^{-3}+\beta(n+1)^{-d / 2} \log n+\beta(n+1)^{-3} \Delta_{n+1}^{\prime}\right], \\
& \left|e_{n+1}^{(N)}(1,3)\right| \leqslant C C_{\theta}^{3}(C \beta)^{N}\left[(n+1)^{-d / 2} \log n+(n+1)^{-3} \Delta_{n+1}^{\prime}\right] .
\end{aligned}
$$

Proof of (8.4). For $N=0$, (8.4) follows from (8.91), (8.92), (8.95) and (8.97). For $N \geqslant 1$, (8.4) follows from (8.91)(8.93), (8.96) and (8.98).

To prove (8.97), (8.98), in view of (8.89), (8.90) and (4.15), we start with $e_{n}^{(0)}\left(\boldsymbol{v}_{N-1} ; \tilde{C}_{N-1} ; 3\right)$. According to (4.50),

$$
\left|e_{n+1}^{(0)}(\boldsymbol{v} ; A ; 3)\right| \leqslant \sum_{b^{\prime} \leqslant\lfloor(n+1) / 2\rfloor} \sum_{b \geqslant b^{\prime}} \mathbb{P}\left(F_{n+1}^{\prime}(\boldsymbol{v} ; A) \cap\left\{b^{\prime} \in \mathcal{P}_{A}\right\} \cap\left\{b \in \mathcal{P}_{A}^{(3)}\right\}\right) .
$$

Let

$$
F_{n+1}^{\prime}\left(\boldsymbol{v}, \boldsymbol{x}, b^{\prime} ; A\right)=E^{\prime}(\boldsymbol{v}, \boldsymbol{x} ; A) \cap F_{n+1}^{\prime}(\boldsymbol{v} ; A) \cap\left\{b^{\prime} \in \mathcal{P}_{A}\right\} .
$$

It follows from (8.99), Proposition 4.10, Lemma 2.2, and the inequality

$$
\mathbb{P}\left(\bar{b} \rightarrow n+1 \text { in } \Lambda \backslash \tilde{C}^{b}(\bar{b})\right) \leqslant \theta_{n+1}(\bar{b})
$$

that 


$$
\begin{aligned}
\left|e_{n+1}^{(0)}(\boldsymbol{v} ; A ; 3)\right| & \leqslant \sum_{b^{\prime} \leqslant\lfloor(n+1) / 2\rfloor} \sum_{b \geqslant b^{\prime}} J_{\underline{b}, \underline{b^{\prime}}} \mathbb{P}\left(F_{n+1}^{\prime}\left(\boldsymbol{v}, \underline{b}, b^{\prime} ; A\right) \text { in } \tilde{C}^{b}(\boldsymbol{v})\right) \theta_{n+1}(\bar{b}) \\
& =\sum_{b^{\prime} \leqslant\lfloor(n+1) / 2\rfloor} \sum_{b \geqslant b^{\prime}} J_{\underline{b}, \overline{b^{\prime}}} \tilde{\mathbb{P}}^{b}\left(F_{n+1}^{\prime}\left(\boldsymbol{v}, \underline{b}, b^{\prime} ; A\right)\right) \theta_{n+1}(\bar{b}),
\end{aligned}
$$

where $\tilde{\mathbb{P}}^{b}$ denotes $\mathbb{P}$ conditioned on $b$ being vacant. By Proposition 4.3,

$$
F_{n+1}^{\prime}(\boldsymbol{v} ; A) \cap\left\{b^{\prime} \in \mathcal{P}_{A}\right\} \subseteq E^{\prime}\left(\boldsymbol{v}, \underline{b}^{\prime} ; A\right) \cap\left\{(\boldsymbol{v} \rightarrow n+1) \circ\left(\left\{b^{\prime} \text { occ. }\right\} \cap\left(\bar{b}^{\prime} \rightarrow n+1\right)\right)\right\},
$$

since, on the right-hand side of (4.25), $(\boldsymbol{v} \rightarrow n+1)$ occurs in $\tilde{C}^{b^{\prime}}(\boldsymbol{v})$, and therefore $(\boldsymbol{v} \rightarrow n+1)$ occurs disjointly from $\left\{b^{\prime}\right.$ occ. $\} \cap\left(\bar{b}^{\prime} \rightarrow n+1\right)$. Then (6.12), (8.100) and (8.103) imply that

$$
F_{n+1}^{\prime}\left(\boldsymbol{v}, \underline{b}, b^{\prime} ; A\right) \subseteq E^{\prime}\left(\boldsymbol{v}, \underline{b}^{\prime} ; A\right) \cap \mathcal{E}(\boldsymbol{v}, \underline{b} ; A) \cap\left\{(\boldsymbol{v} \rightarrow n+1) \circ\left(\left\{b^{\prime} \text { occ. }\right\} \cap\left(\bar{b}^{\prime} \rightarrow n+1\right)\right)\right\} .
$$

The event $\mathcal{E}(\boldsymbol{v}, \underline{b} ; A) \cap\left\{(\boldsymbol{v} \rightarrow n+1) \circ\left(\left\{b^{\prime}\right.\right.\right.$ occ. $\left.\left.\} \cap\left(\bar{b}^{\prime} \rightarrow n+1\right)\right)\right\}$ is increasing, and, for $b^{\prime} \lessgtr b$, the event $E^{\prime}\left(\boldsymbol{v}, \underline{b}^{\prime} ; A\right)$ is independent of the occupation status of $b$. Therefore, by Lemma 7.16,

$$
\begin{aligned}
\left|e_{n+1}^{(0)}(\boldsymbol{v} ; A ; 3)\right| \leqslant \sum_{b^{\prime} \leqslant\lfloor(n+1) / 2\rfloor} \sum_{b \geqslant b^{\prime}} J_{\underline{b}, \bar{b}} \mathbb{P}\left(E^{\prime}\left(\boldsymbol{v}, \underline{b}^{\prime} ; A\right)\right. & \cap \mathcal{E}(\boldsymbol{v}, \underline{b} ; A) \\
\cap\left\{( \boldsymbol { v } \rightarrow n + 1 ) \circ \left(\left\{b^{\prime} \text { occ. }\right\}\right.\right. & \left.\left.\left.\cap\left(\bar{b}^{\prime} \rightarrow n+1\right)\right)\right\}\right) \theta_{n+1}(\bar{b}) .
\end{aligned}
$$

In particular, by (8.89) and (3.20),

$$
\begin{aligned}
\left|e_{n+1}^{(0)}(1,3)\right| \leqslant \sum_{b^{\prime} \leqslant\lfloor(n+1) / 2\rfloor} \sum_{b \geqslant b^{\prime}} J_{\underline{b}, \bar{b}^{\prime}} M_{\underline{b}^{\prime}}^{(1)}(I[\mathcal{E}(\mathbf{0}, \underline{b} ;\{\mathbf{0}\}) \\
\left.\left.\cap\left\{(\mathbf{0} \rightarrow n+1) \circ\left(\left\{b^{\prime} \text { occ. }\right\} \cap\left(\bar{b}^{\prime} \rightarrow n+1\right)\right)\right\}\right]\right) \theta_{n+1}(\bar{b}) .
\end{aligned}
$$

Also, by (8.90), (8.105), (3.20) and (3.35), for $N \geqslant 1$,

$$
\begin{gathered}
\left|e_{n+1}^{(N)}(1,3)\right| \leqslant \sum_{b^{\prime} \leqslant\lfloor(n+1) / 2\rfloor} \sum_{b \geqslant b^{\prime}} \sum_{\left(\boldsymbol{u}_{N-1}, \boldsymbol{v}_{N-1}\right)} J_{\boldsymbol{u}_{N-1}, \boldsymbol{v}_{N-1}} J_{\underline{b}, \bar{b}} M_{\boldsymbol{u}_{N-1}}^{(N)}\left(\mathbb { P } \left(E^{\prime}\left(\boldsymbol{v}_{N-1}, \underline{b}^{\prime} ; \tilde{C}_{N-1}\right)\right.\right. \\
\left.\left.\cap \mathcal{E}\left(\boldsymbol{v}_{N-1}, \underline{b} ; \tilde{C}_{N-1}\right) \cap\left\{\left(\boldsymbol{v}_{N-1} \rightarrow n+1\right) \circ\left(\left\{b^{\prime} \text { occ. }\right\} \cap\left(\bar{b}^{\prime} \rightarrow n+1\right)\right)\right\}\right)\right) \theta_{n+1}(\bar{b}) \\
=\sum_{b^{\prime} \leqslant\lfloor(n+1) / 2\rfloor} \sum_{b \geqslant b^{\prime}} J_{\underline{b}, \bar{b}} M_{\underline{b}^{\prime}}^{(N+1)}\left(I \left[\mathcal{E}\left(\boldsymbol{v}_{N-1}, \underline{b} ; \tilde{C}_{N-1}\right)\right.\right. \\
\left.\left.\cap\left\{\left(\boldsymbol{v}_{N-1} \rightarrow n+1\right) \circ\left(\left\{b^{\prime} \text { occ. }\right\} \cap\left(\bar{b}^{\prime} \rightarrow n+1\right)\right)\right\}\right]\right) \theta_{n+1}(\bar{b}) .
\end{gathered}
$$

We insert

$$
1=I\left[\left(\bar{b}^{\prime} \rightarrow \underline{b}\right)^{c}\right]+I\left[\left(\bar{b}^{\prime} \rightarrow \underline{b}\right)\right]
$$

in the right-hand sides of (8.106), (8.107). We denote the contribution to (8.106) due to the first and second terms of (8.108) by $e_{n+1}^{(0)}(1,3,1)$ and $e_{n+1}^{(0)}(1,3,2)$, respectively. Similarly, for $N \geqslant 1$, we denote the contribution to (8.107) due to the first and second terms of $(8.108)$ by $e_{n+1}^{(N)}(1,3,1)$ and $e_{n+1}^{(N)}(1,3,2)$, respectively.

To estimate $M_{\underline{b^{\prime}}}^{(1)}$ and $M_{\underline{b^{\prime}}}^{(N+1)}$ in (8.106), (8.107), we will use the following proposition, whose proof is deferred to the end of the section. Its statement involves a small modification of $P_{m}^{(N)}(y)$, in which the last Construction $E$ can be applied to all diagram lines rather than only to the $(N-1)$-admissible lines. We denote this modification by $\widetilde{P}_{m}^{(N)}(y)$. The methods in [14] easily adapt to show that the bound (6.2) extends to $\widetilde{P}_{m}^{(N)}(y)$, namely

$$
\sum_{y} \widetilde{P}_{m}^{(N)}(y) \leqslant \delta_{m, 0} \delta_{N, 0}+(C \beta)^{N \vee 1}(m+1)^{-d / 2} .
$$

\section{Proposition 8.11.}

(a) For all bonds $b, \boldsymbol{y} \in \Lambda$ with $m_{\boldsymbol{y}} \geqslant m_{\underline{b}}, N \geqslant 0$, and $n \geqslant 0$, 


$$
\begin{gathered}
M_{\underline{b}}^{(N+1)}\left(I\left[\mathcal{E}\left(\boldsymbol{v}_{N-1}, \boldsymbol{y} ; \tilde{C}_{N-1}\right) \cap(\bar{b} \rightarrow \boldsymbol{y})^{c} \cap\left(\left(\boldsymbol{v}_{N-1} \rightarrow n\right) \circ(\{\text { b occ. }\} \cap(\bar{b} \rightarrow n))\right)\right]\right) \\
\leqslant\left(\left[R^{(N)}(\underline{b}, \boldsymbol{y})+Q^{(N)}(\underline{b}, \boldsymbol{y})\right] \wedge\left[R^{(N)}\left(\underline{b}, \boldsymbol{y} ; \theta_{n}\right)+Q^{(N)}\left(\underline{b}, \boldsymbol{y} ; \theta_{n}\right)\right]\right) J_{\underline{b}, \bar{b}} \theta_{n}(\bar{b}),
\end{gathered}
$$

where, by convention, $Q^{(0)}=0$.

(b) For $\boldsymbol{y} \in \Lambda, N \geqslant 0$, and $n \geqslant 0$,

$$
\begin{aligned}
& \sum_{b} M_{\underline{b}}^{(N+1)}\left(I\left[\mathcal{E}\left(\boldsymbol{v}_{N-1}, \boldsymbol{y} ; \tilde{C}_{N-1}\right) \cap(\bar{b} \rightarrow \boldsymbol{y}) \cap\left(\left(\boldsymbol{v}_{N-1} \rightarrow n\right) \circ(\{\text { occ. }\} \cap(\bar{b} \rightarrow n))\right)\right]\right) \\
& \quad \leqslant \widetilde{P}^{(N+1)}(\boldsymbol{y}) \wedge \widetilde{P}^{(N+1)}\left(\boldsymbol{y} ; \theta_{n}, \theta_{n}\right) .
\end{aligned}
$$

Before proving Proposition 8.11, we first prove (8.97), (8.98).

Proof of (8.97). By the definition below (8.108), $e_{n+1}^{(0)}(1,3)=e_{n+1}^{(0)}(1,3,1)+e_{n+1}^{(0)}(1,3,2)$. By (8.106) and Proposition 8.11(a) (with $N=0$, and $\boldsymbol{y}$ and $b$ in (8.110) equal to $\underline{b}$ and $b^{\prime}$ in (8.106)),

$$
\begin{aligned}
\left|e_{n+1}^{(0)}(1,3,1)\right| & \leqslant \sum_{b^{\prime} \leqslant\lfloor(n+1) / 2\rfloor} \sum_{b \ngtr b^{\prime}} J_{\underline{b}, \bar{b}} J_{\underline{b}^{\prime}, \bar{b}^{\prime}}\left[R^{(0)}\left(\underline{b}^{\prime}, \underline{b}\right) \wedge R^{(0)}\left(\underline{b}^{\prime}, \underline{b} ; \theta_{n+1}\right)\right] \theta_{n+1}(\bar{b}) \theta_{n+1}\left(\bar{b}^{\prime}\right) \\
& =p_{c}^{2} \sum_{m_{1}=0}^{\lfloor n / 2\rfloor} \sum_{m_{2}=m_{1}}^{n}\left[R_{m_{1}, m_{2}}^{(0)} \wedge R_{m_{1}, m_{2}}^{(0)}\left(\theta_{n+1}\right)\right] \theta_{n-m_{1}} \theta_{n-m_{2}},
\end{aligned}
$$

where $R_{m_{1}, m_{2}}^{(0)}=\sum_{y_{1}, y_{2}} R_{m_{1}, m_{2}}^{(0)}\left(y_{1}, y_{2}\right)$. By Proposition 7.7 (with additional attention paid to the power of $\beta$ ),

$$
R_{m_{1}, m_{2}}^{(0)} \leqslant C \delta_{m_{1}, 0} \delta_{m_{2}, 0}+C \beta b_{m_{1}, m_{2}} .
$$

The contribution due to $\left(m_{1}, m_{2}\right)=(0,0)$ gives rise to at most $C C_{\theta}^{3}(n+1)^{-3}$. For the contribution due to $\left(m_{1}, m_{2}\right) \neq$ $(0,0)$, we apply Lemma 8.6 to obtain a bound

$$
C C_{\theta}^{3} \beta\left[(n+1)^{-d / 2} \log n+(n+1)^{-3} \Delta_{n+1}^{\prime}\right] .
$$

Adding the two contributions yields

$$
\left|e_{n+1}^{(0)}(1,3,1)\right| \leqslant C C_{\theta}^{3}\left[(n+1)^{-3}+\beta(n+1)^{-d / 2} \log n+\beta(n+1)^{-3} \Delta_{n+1}^{\prime}\right] .
$$

Similarly, by Proposition 8.11(b) (with $N=0, \boldsymbol{y}=\underline{b}=\left(y_{2}, m_{2}\right)$, and the summation index $b$ in (8.111) equal to $b^{\prime}$ in (8.106)),

$$
\begin{aligned}
\left|e_{n+1}^{(0)}(1,3,2)\right| & \leqslant \sum_{b} J_{\underline{b}, \bar{b}}\left[\widetilde{P}^{(1)}(\underline{b}) \wedge \widetilde{P}^{(1)}\left(\underline{b} ; \theta_{n+1}, \theta_{n+1}\right)\right] \theta_{n+1}(\bar{b}) \\
& =p_{c} \sum_{m_{2}=0}^{n}\left[\widetilde{P}_{m_{2}}^{(1)} \wedge \widetilde{P}_{m_{2}}^{(1)}\left(\theta_{n+1}, \theta_{n+1}\right)\right] \theta_{n-m_{2}}
\end{aligned}
$$

where we write $\widetilde{P}_{m_{2}}^{(1)}=\sum_{y_{2}} \widetilde{P}_{m_{2}}^{(1)}\left(y_{2}\right)$. By (8.109) and Corollary 8.4, we therefore obtain

$$
\left|e_{n+1}^{(0)}(1,3,2)\right| \leqslant C C_{\theta}^{3} \beta\left[(n+1)^{-3} \Delta_{n+1}^{\prime}+\beta(n+1)^{-d / 2} \log n\right] .
$$

Addition of (8.115) and (8.117) gives (8.97).

Proof of (8.98). By (8.107) and Proposition 8.11(a),

$$
\left|e_{n+1}^{(N)}(1,3,1)\right| \leqslant p_{c}^{2} \sum_{m_{1}=0}^{\lfloor n / 2\rfloor} \sum_{m_{2}=m_{1}}^{n}\left(\left[R_{m_{1}, m_{2}}^{(N)}+Q_{m_{1}, m_{2}}^{(N)}\right] \wedge\left[R_{m_{1}, m_{2}}^{(N)}\left(\theta_{n+1}\right)+Q_{m_{1}, m_{2}}^{(N)}\left(\theta_{n+1}\right)\right]\right) \theta_{n-m_{1}} \theta_{n-m_{2}} .
$$

By Proposition 7.7 and Lemma 8.6, it follows that

$$
\left|e_{n+1}^{(N)}(1,3,1)\right| \leqslant C C_{\theta}^{3}(C \beta)^{N}\left[(n+1)^{-d / 2} \log n+(n+1)^{-3} \Delta_{n+1}^{\prime}\right] .
$$


Similarly, by Proposition 8.11(b) (with $\boldsymbol{y}=\underline{b}=\left(y_{2}, m_{2}\right)$, and the summation index $b$ in (8.111) equal to $b^{\prime}$ in (8.107)),

$$
\left|e_{n+1}^{(N)}(1,3,2)\right| \leqslant p_{c} \sum_{m_{2}=0}^{n}\left[\widetilde{P}_{m_{2}}^{(N+1)} \wedge \widetilde{P}_{m_{2}}^{(N+1)}\left(\theta_{n+1}, \theta_{n+1}\right)\right] \theta_{n-m_{2}} .
$$

It then follows from (8.109) and Corollary 8.4 that

$$
\left|e_{n+1}^{(N)}(1,3,2)\right| \leqslant C C_{\theta}^{3}(C \beta)^{N}\left[(n+1)^{-d / 2} \log n+(n+1)^{-3} \Delta_{n+1}^{\prime}\right] .
$$

Addition of (8.119) and (8.121) gives (8.98).

It remains to prove Proposition 8.11. For this, we will use the following lemma.

Lemma 8.12. For any bond $b, \boldsymbol{v}, \boldsymbol{y} \in \Lambda, A \subseteq \Lambda, n \geqslant 0$,

$$
\begin{aligned}
& \mathcal{E}(\boldsymbol{v}, \boldsymbol{y} ; A) \cap(\bar{b} \rightarrow \boldsymbol{y})^{c} \cap((\boldsymbol{v} \rightarrow n) \circ(\{b \text { occ. }\} \cap(\bar{b} \rightarrow n))) \\
& \quad \subseteq(\mathcal{E}(\boldsymbol{v}, \boldsymbol{y} ; A) \cap(\boldsymbol{v} \rightarrow n)) \circ(\{b \text { occ. }\} \cap(\bar{b} \rightarrow n)), \\
& \mathcal{E}(\boldsymbol{v}, \boldsymbol{y} ; A) \cap(\bar{b} \rightarrow \boldsymbol{y}) \cap\{b \text { occ. }\} \subseteq((\{b \text { occ }\} \cap(\bar{b} \rightarrow \boldsymbol{y})) \circ(\boldsymbol{v} \rightarrow \boldsymbol{y})) \cup(\{b \text { occ. }\} \cap \mathcal{E}(\bar{b}, \boldsymbol{y} ; A)) .
\end{aligned}
$$

Proof. To prove (8.122), we note that

$$
\mathcal{E}(\boldsymbol{v}, \boldsymbol{y} ; A) \cap(\bar{b} \rightarrow \boldsymbol{y})^{c} \cap\{b \text { occ. }\} \cap(\bar{b} \rightarrow n) \subseteq \mathcal{E}(\boldsymbol{v}, \boldsymbol{y} ; A) \circ(\{b \text { occ. }\} \cap(\bar{b} \rightarrow n)),
$$

since, when $(\bar{b} \rightarrow \boldsymbol{y})^{c}$ occurs, $b$ and the connections from $\bar{b}$ cannot be required in the event $\mathcal{E}(\boldsymbol{v}, \boldsymbol{y} ; A)$. Therefore,

$$
\begin{aligned}
\mathcal{E} & (\boldsymbol{v}, \boldsymbol{y} ; A) \cap(\bar{b} \rightarrow \boldsymbol{y})^{c} \cap((\boldsymbol{v} \rightarrow n) \circ(\{b \text { occ. }\} \cap(\bar{b} \rightarrow n))) \\
& =\left(\mathcal{E}(\boldsymbol{v}, \boldsymbol{y} ; A) \cap(\bar{b} \rightarrow \boldsymbol{y})^{c} \cap\{b \text { occ. }\} \cap(\bar{b} \rightarrow n)\right) \cap((\boldsymbol{v} \rightarrow n) \circ(\{b \text { occ. }\} \cap(\bar{b} \rightarrow n))) \\
& \subseteq(\mathcal{E}(\boldsymbol{v}, \boldsymbol{y} ; A) \circ(\{b \text { occ. }\} \cap(\bar{b} \rightarrow n))) \cap((\boldsymbol{v} \rightarrow n) \circ(\{b \text { occ. }\} \cap(\bar{b} \rightarrow n))) \cap(\bar{b} \rightarrow \boldsymbol{y})^{c} \\
& \subseteq(\mathcal{E}(\boldsymbol{v}, \boldsymbol{y} ; A) \cap(\boldsymbol{v} \rightarrow n)) \circ(\{b \text { occ. }\} \cap(\bar{b} \rightarrow n)),
\end{aligned}
$$

where, in the last step, we used $(\bar{b} \rightarrow \boldsymbol{y})^{c}$ to conclude that the occupied path from $\bar{b}$ to $n$ that is disjoint from $(\boldsymbol{v} \rightarrow n)$ is also disjoint from a set of paths realising $\mathcal{E}(\boldsymbol{v}, \boldsymbol{y} ; A)$. This proves (8.122).

Next, we prove (8.123). If there is no pivotal bond for $\boldsymbol{v} \rightarrow \boldsymbol{y}$, then $\boldsymbol{v} \Rightarrow \boldsymbol{y}$, and thus $(\{b$ occ. $\} \cap(\bar{b} \rightarrow \boldsymbol{y})) \circ(\boldsymbol{v} \rightarrow \boldsymbol{y})$ occurs. This leaves the case where there is a pivotal bond for $\boldsymbol{v} \rightarrow \boldsymbol{y}$, and in this case, we let $b^{\prime}$ denote the last pivotal bond. If the left-hand side of (8.123) occurs and $\bar{b} \rightarrow \underline{b}^{\prime}$, then $b$ is occupied and $\mathcal{E}(\bar{b}, \boldsymbol{y} ; A)$ occurs (since there is a vertex in $A$ on one of the paths from $\bar{b}^{\prime}$ to $\left.\boldsymbol{y}\right)$. If, on the other hand, the left-hand side of (8.123) and $\left(\bar{b} \rightarrow \underline{b}^{\prime}\right)^{c}$ both occur, then $(\{b$ occ. $\} \cap(\bar{b} \rightarrow \boldsymbol{y})) \circ(\boldsymbol{v} \rightarrow \boldsymbol{y})$ occurs, since $(\{b$ occ. $\} \cap(\bar{b} \rightarrow \boldsymbol{y})) \circ\left(\bar{b}^{\prime} \rightarrow \boldsymbol{y}\right)$ occurs as in the case of no pivotal bond and since the connection from $v$ to $\bar{b}^{\prime}$ must also be disjoint from $(\{b$ occ. $\} \cap(\bar{b} \rightarrow \boldsymbol{y}))$. This proves (8.123).

Proof of Proposition 8.11. (a) By (8.122) and (3.35),

$$
\begin{aligned}
M_{\underline{b}}^{(N+1)}\left(I\left[\mathcal{E}\left(\boldsymbol{v}_{N-1}, \boldsymbol{y} ; \tilde{C}_{N-1}\right) \cap(\bar{b} \rightarrow \boldsymbol{y})^{c} \cap\left(\left(\boldsymbol{v}_{N-1} \rightarrow n\right) \circ(\{b \text { occ. }\} \cap \bar{b} \rightarrow n)\right)\right]\right) \\
\leqslant M_{\underline{b}}^{(N+1)}\left(I\left[\left(\mathcal{E}\left(\boldsymbol{v}_{N-1}, \boldsymbol{y} ; \tilde{C}_{N-1}\right) \cap(\boldsymbol{v} \rightarrow n)\right) \circ(\{b \text { occ. }\} \cap(\bar{b} \rightarrow n))\right]\right) \\
=\sum_{\left(\boldsymbol{u}_{N-1}, \boldsymbol{v}_{N-1}\right)} J_{\boldsymbol{u}_{N-1}, \boldsymbol{v}_{N-1}} M_{\boldsymbol{u}_{N-1}}^{(N)}\left(\mathbb { P } \left(E^{\prime}\left(\boldsymbol{v}_{N-2}, \underline{b} ; \tilde{C}_{N-2}\right)\right.\right. \\
\left.\left.\quad \cap\left(\left(\mathcal{E}\left(\boldsymbol{v}_{N-1}, \boldsymbol{y} ; \tilde{C}_{N-1}\right) \cap\left(\boldsymbol{v}_{N-1} \rightarrow n\right)\right) \circ(\{b \text { occ. }\} \cap(\bar{b} \rightarrow n))\right)\right)\right),
\end{aligned}
$$

where we assume for convenience in this paragraph that $N \geqslant 1$ (the case $N=0$ is similar). Due to the orientation of the bonds, the events on the right-hand side of (8.126) can be rewritten as

$$
\begin{aligned}
& E^{\prime}\left(\boldsymbol{v}_{N-2}, \underline{b} ; \tilde{C}_{N-2}\right) \cap\left(\mathcal{E}\left(\boldsymbol{v}_{N-1}, \boldsymbol{y} ; \tilde{C}_{N-1}\right) \cap\left(\boldsymbol{v}_{N-1} \rightarrow n\right)\right) \circ(\{b \text { occ. }\} \cap(\bar{b} \rightarrow n)) \\
& \quad=\left(E^{\prime}\left(\boldsymbol{v}_{N-2}, \underline{b} ; \tilde{C}_{N-2}\right) \cap \mathcal{E}\left(\boldsymbol{v}_{N-1}, \boldsymbol{y} ; \tilde{C}_{N-1}\right) \cap\left(\boldsymbol{v}_{N-1} \rightarrow n\right)\right) \circ(\{b \text { occ. }\} \cap(\bar{b} \rightarrow n)) .
\end{aligned}
$$


By the BKR inequality,

$$
\begin{aligned}
& \mathbb{P}\left(\left(E^{\prime}\left(\boldsymbol{v}_{N-2}, \underline{b} ; \tilde{C}_{N-2}\right) \cap \mathcal{E}\left(\boldsymbol{v}_{N-1}, \boldsymbol{y} ; \tilde{C}_{N-1}\right) \cap\left(\boldsymbol{v}_{N-1} \rightarrow n\right)\right) \circ(\{b \text { occ. }\} \cap(\bar{b} \rightarrow n))\right) \\
& \quad \leqslant \mathbb{P}\left(E^{\prime}\left(\boldsymbol{v}_{N-2}, \underline{b} ; \tilde{C}_{N-2}\right) \cap \mathcal{E}\left(\boldsymbol{v}_{N-1}, \boldsymbol{y} ; \tilde{C}_{N-1}\right) \cap\left(\boldsymbol{v}_{N-1} \rightarrow n\right)\right) J_{\underline{b}, \bar{b}} \theta_{n}(\bar{b}) .
\end{aligned}
$$

We then substitute (8.128) into (8.126), and again use (3.35), to see that

$$
\begin{aligned}
& M_{\underline{b}}^{(N+1)}\left(I\left[\mathcal{E}\left(\boldsymbol{v}_{N-1}, \boldsymbol{y} ; \tilde{C}_{N-1}\right) \cap(\bar{b} \rightarrow \boldsymbol{y})^{c} \cap\left(\left(\boldsymbol{v}_{N-1} \rightarrow n\right) \circ(\{b \text { occ. }\} \cap \bar{b} \rightarrow n)\right)\right]\right) \\
& \quad \leqslant M_{\underline{b}}^{(N+1)}\left(I\left[\mathcal{E}\left(\boldsymbol{v}_{N-1}, \boldsymbol{y} ; \tilde{C}_{N-1}\right) \cap\left(\boldsymbol{v}_{N-1} \rightarrow n\right)\right]\right) J_{\underline{b}, \bar{b}} \theta_{n}(\bar{b}) .
\end{aligned}
$$

Since $m_{y} \geqslant m_{\underline{b}}$, we can use the definitions in (6.12) and (7.47), (7.48) to see that

$$
I\left[\mathcal{E}\left(\boldsymbol{v}_{N-1}, \boldsymbol{y} ; \tilde{C}_{N-1}\right)\right] \leqslant I\left[V_{m_{\underline{b}}}\left(\boldsymbol{v}_{N-1}, \boldsymbol{y}\right)\right]+I\left[\mathcal{E}_{m_{\underline{b}}+1}\left(\boldsymbol{v}_{N-1}, \boldsymbol{y} ; \tilde{C}_{N-1}\right)\right],
$$

where, for $N=0$, the second contribution is identically zero. If we bound $I\left[\left(\boldsymbol{v}_{N-1} \rightarrow n\right)\right]$ by 1 in $(8.129)$, it then follows from Lemma 7.13 that the contribution due to $I\left[V_{m_{\underline{b}}}\left(\boldsymbol{v}_{N-1}, \boldsymbol{y}\right)\right]$ is bounded by $P^{(N)}\left(\underline{b} ; V_{m_{b}}(\boldsymbol{y})\right)=R^{(N)}(\underline{b}, \boldsymbol{y})$. Also, by Lemma 7.14 , the contribution due to $I\left[\mathcal{E}_{m_{\underline{b}}+1}\left(\boldsymbol{v}_{N-1}, \boldsymbol{y} ; \tilde{C}_{N-1}\right)\right]$ is bounded by $P^{\underline{(}(N)}\left(\underline{b} ; \mathcal{E}_{m_{\underline{b}}}+1(\boldsymbol{y})\right)=$ $Q^{(N)}(\underline{b}, \boldsymbol{y})$. Adding these, we obtain

$$
M_{\underline{b}}^{(N+1)}\left(I\left[\mathcal{E}\left(\boldsymbol{v}_{N-1}, \boldsymbol{y} ; \tilde{C}_{N-1}\right)\right]\right) \leqslant R^{(N)}(\underline{b}, \boldsymbol{y})+Q^{(N)}(\underline{b}, \boldsymbol{y}),
$$

which gives the upper bound $\left[R^{(N)}(\underline{b}, \boldsymbol{y})+Q^{(N)}(\underline{b}, \boldsymbol{y})\right] J_{\underline{b}, \bar{b}} \theta_{n}(\bar{b})$ of (8.110). Also, the bounds

$$
\begin{aligned}
& M_{\underline{b}}^{(N+1)}\left(I\left[V_{m_{\underline{b}}}\left(\boldsymbol{v}_{N-1}, \boldsymbol{y} ; \tilde{C}_{N-1}\right) \cap\left(\boldsymbol{v}_{N-1} \rightarrow n\right)\right]\right) \leqslant R^{(N)}\left(\underline{b}, \boldsymbol{y} ; \theta_{n}\right), \\
& M_{\underline{b}}^{(N+1)}\left(I\left[\mathcal{E}_{m_{\underline{b}}+1}\left(\boldsymbol{v}_{N-1}, \boldsymbol{y} ; \tilde{C}_{N-1}\right) \cap\left(\boldsymbol{v}_{N-1} \rightarrow n\right)\right]\right) \leqslant Q^{(N)}\left(\underline{b}, \boldsymbol{y} ; \theta_{n}\right)
\end{aligned}
$$

are minor adaptations of (7.58) and (7.63). This proves (a).

(b) We bound the contributions to (8.111) due to both terms in (8.123) separately. Using (3.35), the contribution to $(8.111)$ due to the first term on the right-hand side of $(8.123)$ is

$$
\begin{aligned}
M_{\underline{b}}^{(N+1)} & \left(I\left[\left((\{b \text { occ. }\} \cap(\bar{b} \rightarrow \boldsymbol{y})) \circ\left(\boldsymbol{v}_{N-1} \rightarrow \boldsymbol{y}\right)\right) \cap\left(\left(\boldsymbol{v}_{N-1} \rightarrow n\right) \circ(\bar{b} \rightarrow n)\right)\right]\right) \\
= & \sum_{\left(\boldsymbol{u}_{N-1}, \boldsymbol{v}_{N-1}\right)} J_{\boldsymbol{u}_{N-1}, \boldsymbol{v}_{N-1}} M_{\boldsymbol{u}_{N-1}}^{(N)}\left(M _ { \boldsymbol { v } _ { N - 1 } , \underline { b } , \tilde { C } _ { N - 1 } } ^ { ( 1 ) } \left(I \left[\left((\{b \text { occ. }\} \cap(\bar{b} \rightarrow \boldsymbol{y})) \circ\left(\boldsymbol{v}_{N-1} \rightarrow \boldsymbol{y}\right)\right)\right.\right.\right. \\
& \left.\left.\left.\cap\left(\left(\boldsymbol{v}_{N-1} \rightarrow n\right) \circ(\bar{b} \rightarrow n)\right)\right]\right)\right) .
\end{aligned}
$$

We temporarily omit the two connections to $n$ inside the above expression, and observe that

$$
\begin{aligned}
& \sum_{b} M_{\boldsymbol{v}_{N-1}, \underline{b} ; \tilde{C}_{N-1}}^{(1)}\left(I\left[(\{b \text { occ. }\} \cap(\bar{b} \rightarrow \boldsymbol{y})) \circ\left(\boldsymbol{v}_{N-1} \rightarrow \boldsymbol{y}\right)\right]\right) \\
& \leqslant P^{(0)}\left(\boldsymbol{v}_{N-1}, \underline{b} ; \tilde{C}_{N-1}, 2_{\underline{b}}^{(1)}(\boldsymbol{y})\right) \leqslant P^{(1)}\left(\boldsymbol{v}_{N-1}, \boldsymbol{y} ; \tilde{C}_{N-1}\right) .
\end{aligned}
$$

Thus, once we take into account the two connections to $n$, we obtain

$$
\begin{aligned}
& \sum_{b} M_{\boldsymbol{v}_{N-1}, \underline{b} ; \tilde{C}_{N-1}}^{(1)}\left(I\left[\left((\{b \text { occ. }\} \cap(\bar{b} \rightarrow \boldsymbol{y})) \circ\left(\boldsymbol{v}_{N-1} \rightarrow \boldsymbol{y}\right)\right) \cap\left(\left(\boldsymbol{v}_{N-1} \rightarrow n\right) \circ(\bar{b} \rightarrow n)\right)\right]\right) \\
& \quad \leqslant P^{(1)}\left(\boldsymbol{v}_{N-1}, \boldsymbol{y} ; \tilde{C}_{N-1}, \theta_{n}, \theta_{n}\right) \wedge P^{(1)}\left(\boldsymbol{v}_{N-1}, \boldsymbol{y} ; \tilde{C}_{N-1}\right) .
\end{aligned}
$$

We substitute (8.136) into the summation of Eq. (8.134) over $b$. With (6.11) and (6.21), this gives

$$
\begin{aligned}
& \sum_{b} M_{\underline{b}}^{(N+1)}\left(I\left[\mathcal{E}\left(\boldsymbol{v}_{N-1}, \boldsymbol{y} ; \tilde{C}_{N-1}\right) \cap\{b \text { occ. }\} \cap(\bar{b} \rightarrow \boldsymbol{y}) \cap\left(\left(\boldsymbol{v}_{N-1} \rightarrow n\right) \circ(\bar{b} \rightarrow n)\right)\right]\right) \\
& \quad \leqslant \sum_{\left(\boldsymbol{u}_{N-1}, \boldsymbol{v}_{N-1}\right)} J_{\boldsymbol{u}_{N-1}, \boldsymbol{v}_{N-1}} M_{\boldsymbol{u}_{N-1}^{(N)}}^{\left(N^{\prime}\right)}\left(P^{(1)}\left(\boldsymbol{v}_{N-1}, \boldsymbol{y} ; \tilde{C}_{N-1}, \theta_{n}, \theta_{n}\right) \wedge P^{(1)}\left(\boldsymbol{v}_{N-1}, \boldsymbol{y} ; \tilde{C}_{N-1}\right)\right) \\
& \quad \leqslant P^{(N+1)}\left(\boldsymbol{y} ; \theta_{n}, \theta_{n}\right) \wedge P^{(N+1)}(\boldsymbol{y}) .
\end{aligned}
$$


The obvious inequality $P^{(N+1)} \leqslant \widetilde{P}^{(N+1)}$ completes the analysis for the first term in (8.123).

For the contribution to (8.111) due to second term in (8.123), (3.35) gives

$$
\begin{aligned}
& M_{\underline{b}}^{(N+1)}\left(I\left[\mathcal{E}\left(\bar{b}, \boldsymbol{y} ; \tilde{C}_{N-1}\right) \cap\{b \text { occ. }\} \cap\left(\left(\boldsymbol{v}_{N-1} \rightarrow n\right) \circ(\bar{b} \rightarrow n)\right)\right]\right) \\
& =\sum_{\left(\boldsymbol{u}_{N-1}, \boldsymbol{v}_{N-1}\right)} J_{\boldsymbol{u}_{N-1}, \boldsymbol{v}_{N-1}} \\
& \quad \times M_{\boldsymbol{u}_{N-1}}^{(N)}\left(M_{\boldsymbol{v}_{N-1}, \underline{b} ; \tilde{C}_{N-1}}^{(1)}\left(I\left[\mathcal{E}\left(\bar{b}, \boldsymbol{y} ; \tilde{C}_{N-1}\right) \cap\{b \text { occ. }\} \cap\left(\left(\boldsymbol{v}_{N-1} \rightarrow n\right) \circ(\bar{b} \rightarrow n)\right)\right]\right)\right) .
\end{aligned}
$$

By (6.13),

$$
\begin{aligned}
& M_{\boldsymbol{v}_{N-1}, \underline{b} ; \tilde{C}_{N-1}}^{(1)}\left(I\left[\mathcal{E}\left(\bar{b}, \boldsymbol{y} ; \tilde{C}_{N-1}\right) \cap\{b \text { occ. }\} \cap\left(\left(\boldsymbol{v}_{N-1} \rightarrow n\right) \circ(\bar{b} \rightarrow n)\right)\right]\right) \\
& \quad \leqslant \mathbb{P}\left(\mathcal{E}\left(\boldsymbol{v}_{N-1}, \underline{b} ; \tilde{C}_{N-1}\right) \cap\{b \text { occ. }\} \cap \mathcal{E}\left(\bar{b}, \boldsymbol{y} ; \tilde{C}_{N-1}\right) \cap\left(\left(\boldsymbol{v}_{N-1} \rightarrow n\right) \circ(\bar{b} \rightarrow n)\right)\right) .
\end{aligned}
$$

We again temporarily omit the two connections to $n$ inside the above expression, and observe that, by the Markov property,

$$
\mathbb{P}(\mathcal{E}(\boldsymbol{v}, \underline{b} ; A) \cap\{b \text { occ. }\} \cap \mathcal{E}(\bar{b}, \boldsymbol{y} ; A))=J_{\underline{b}, \bar{b}} P^{(0)}(\boldsymbol{v}, \underline{b} ; A) P^{(0)}(\bar{b}, \boldsymbol{y} ; A),
$$

which leads to an upper bound

$$
\sum_{b} \sum_{\left(\boldsymbol{u}_{N-1}, \boldsymbol{v}_{N-1}\right)} J_{\boldsymbol{u}_{N-1}, \boldsymbol{v}_{N-1}} M_{\boldsymbol{u}_{N-1}}^{(N)}\left(\mathbb{P}_{N}\left(\mathcal{E}\left(\boldsymbol{v}_{N-1}, \underline{b} ; \tilde{C}_{N-1}\right) \cap\{b \text { occ. }\} \cap \mathcal{E}\left(\bar{b}, \boldsymbol{y} ; \tilde{C}_{N-1}\right)\right)\right) \leqslant \widetilde{P}^{(N+1)}(\boldsymbol{y}) .
$$

Note that $\widetilde{P}^{(N+1)}$ occurs, rather than $P^{(N+1)}$, due to the fact that the two events $\mathcal{E}\left(\boldsymbol{v}_{N-1}, \underline{b} ; \tilde{C}_{N-1}\right)$ and $\mathcal{E}\left(\bar{b}, \boldsymbol{y} ; \tilde{C}_{N-1}\right)$ depend on the same set of vertices $\tilde{C}_{N-1}$, whereas, for $P^{(N+1)}$, the event $\mathcal{E}\left(\bar{b}, \boldsymbol{y} ; \tilde{C}_{N-1}\right)$ would be replaced by $\mathcal{E}\left(\bar{b}, \boldsymbol{y} ; \tilde{C}^{b}\left(\boldsymbol{v}_{N-1}\right)\right)$. This change can be bounded by letting the last Construction $E$ be applied to all lines, rather than only the $N$-admissible lines. It is not difficult to see that if we now take into account the two connections to $n$, then we obtain

$$
\begin{aligned}
& \sum_{b} M_{\underline{b}}^{(N+1)}\left(I\left[\mathcal{E}\left(\boldsymbol{v}_{N-1}, \boldsymbol{y} ; \tilde{C}_{N-1}\right) \cap\{b \text { occ. }\} \cap(\bar{b} \rightarrow \boldsymbol{y}) \cap\left(\left(\boldsymbol{v}_{N-1} \rightarrow n\right) \circ(\bar{b} \rightarrow n)\right)\right]\right) \\
& \quad \leqslant \widetilde{P}^{(N+1)}\left(\boldsymbol{y} ; \theta_{n}, \theta_{n}\right) \wedge \widetilde{\boldsymbol{P}}^{(N+1)}(\boldsymbol{y}) .
\end{aligned}
$$

Together, (8.137) and (8.142) prove (8.111).

\section{Acknowledgements}

The work of RvdH was supported in part by the Netherlands Organisation for Scientific Research (NWO), and was performed in part while visiting Microsoft Research in July 2004. The work of GS was supported in part by NSERC of Canada and by the Thomas Stieltjes Institute in The Netherlands. RvdH and FdH thank the University of British Columbia, Vancouver, Canada, and GS thanks EURANDOM, Eindhoven, The Netherlands, for hospitality. The authors thank Antal Járai for conversations during the early stages of this work, and Akira Sakai for several stimulating conversations later on.

\section{References}

[1] D.J. Barsky, M. Aizenman, Percolation critical exponents under the triangle condition, Ann. Probab. 19 (1991) $1520-1536$.

[2] C. Bezuidenhout, G. Grimmett, The critical contact process dies out, Ann. Probab. 18 (1990) 1462-1482.

[3] G. Grimmett, Percolation, second ed., Springer, Berlin, 1999.

[4] G. Grimmett, P. Hiemer, Directed percolation and random walk, in: V. Sidoravicius (Ed.), In and Out of Equilibrium, Birkhäuser, Boston, 2002, pp. 273-297.

[5] T. Hara, G. Slade, Mean-field critical behaviour for percolation in high dimensions, Comm. Math. Phys. 128 (1990) $333-391$.

[6] T. Hara, G. Slade, The scaling limit of the incipient infinite cluster in high-dimensional percolation. I. Critical exponents, J. Stat. Phys. 99 (2000) 1075-1168.

[7] R. van der Hofstad, F. den Hollander, G. Slade, The survival probability for critical spread-out oriented percolation above $4+1$ dimensions. I. Induction. Preprint, 2005. Probab. Theory Related Fields, in press. 
[8] R. van der Hofstad, F. den Hollander, G. Slade, Construction of the incipient infinite cluster for spread-out oriented percolation above $4+1$ dimensions, Comm. Math. Phys. 231 (2002) 435-461.

[9] R. van der Hofstad, A. Sakai, Gaussian scaling for the critical spread-out contact process above the upper critical dimension, Electron. J. Probab. 9 (2004) 710-769.

[10] R. van der Hofstad, A. Sakai, Critical points for spread-out self-avoiding walk, percolation and the contact process, Probab. Theory Related Fields 132 (2005) 438-470.

[11] R. van der Hofstad, A. Sakai, Convergence of the critical finite-range contact process to super-Brownian motion above the upper critical dimension. I. The higher-point functions, in preparation.

[12] R. van der Hofstad, A. Sakai, Convergence of the critical finite-range contact process to super-Brownian motion above the upper critical dimension. II. The survival probability, in preparation.

[13] R. van der Hofstad, G. Slade, A generalised inductive approach to the lace expansion, Probab. Theory Related Fields 122 (2002) 389-430.

[14] R. van der Hofstad, G. Slade, Convergence of critical oriented percolation to super-Brownian motion above $4+1$ dimensions, Ann. Inst. H. Poincaré Probab. Statist. 39 (2003) 415-485.

[15] N. Madras, G. Slade, The Self-Avoiding Walk, Birkhäuser, Boston, 1993.

[16] B.G. Nguyen, W.-S. Yang, Triangle condition for oriented percolation in high dimensions, Ann. Probab. 21 (1993) 1809-1844.

[17] A. Sakai, Mean-field critical behavior for the contact process, J. Stat. Phys. 104 (2001) 111-143.

[18] G. Slade, The Lace Expansion and its Applications, Lecture Notes in Mathematics, vol. 1879, Springer, 2006, Ecole d'Eté Probabilit. SaintFlour. 